Illinois State University

ISU ReD: Research and eData

Theses and Dissertations

$4-6-2018$

\title{
Alkyne Combustion: Experimental and Computational Studies of Formyl Radical Production
}

Matthew Charles Drummer

Illinois State University, mcdrumm@ilstu.edu

Follow this and additional works at: https://ir.library.illinoisstate.edu/etd

Part of the Physical Chemistry Commons

\section{Recommended Citation}

Drummer, Matthew Charles, "Alkyne Combustion: Experimental and Computational Studies of Formyl Radical Production" (2018). Theses and Dissertations. 853.

https://ir.library.illinoisstate.edu/etd/853

This Thesis is brought to you for free and open access by ISU ReD: Research and eData. It has been accepted for inclusion in Theses and Dissertations by an authorized administrator of ISU ReD: Research and eData. For more information, please contact ISUReD@ilstu.edu. 


\title{
ALKYNE COMBUSTION: EXPERIMENTAL AND COMPUTATIONAL STUDIES OF FORMYL RADICAL PRODUCTION
}

\author{
Matthew C. Drummer
}

\section{Pages}

According to the U.S. Energy Information Administration, the United States consumed a total of 97.4 trillion BTUs (British Thermal Unit, $1 \mathrm{BTU}=1.055 \mathrm{~kJ}$ ) of energy in 2016 with over $80 \%$ of that energy consumption source being fossil fuel combustion. Before a combustion reaction reaches its end products, a number of intermediate products form and may react with other abundant atmospheric species to form aerosol particles and acid rain, both of which have potentially negative impacts on both human-made structures and the natural environment.

In an effort to counteract the consequences of fossil fuel combustion, scientists are interested in gaining insight into the reaction mechanisms of hydrocarbon combustion reactions to understand how, and which, intermediate products form. One of the many intermediate products formed in hydrocarbon combustion reactions is the formyl radical, HCO. Discovered in 1934, HCO radical has since been proven to form in many hundreds of combustion reactions and plays key roles in both atmospheric and interstellar chemistry, namely as a donor of hydroxyl radicals.

While alkane combustion has been studied extensively in the past, alkene and alkyne combustion has received little attention beyond the short-chain species: ethylene, propene, acetylene, and propyne. Due to receiving little attention, the formation of HCO radical during alkyne combustion reactions is focused on in this project. This thesis provides results from both 
experimental and theoretical studies on the reactions of triplet oxygen atoms, $\mathrm{O}\left({ }^{3} \mathrm{P}\right)$, with the hydroxyl-substituted alkynes, propargyl alcohol and 3-butyn-1-ol.

Preliminary experimental studies of alkyne combustion revealed strong variations in HCO radical absorption intensity across different alkyne species. The most dramatic difference in HCO radical absorption intensity was observed between the combustion of propyne and propargyl alcohol, where propargyl alcohol gave a much stronger signal for HCO radical than propyne. Both compounds are three-carbon alkynes, with the only difference being the hydroxyl substituent of propargyl alcohol. This study attempts to explain these variations via computational investigation. Utilizing quantum mechanical methods, potential energy profiles have been mapped out to reveal the energetics of reaction pathways that result in the formation of $\mathrm{HCO}$ radical. Isoformyl radical, HOC, is also investigated computationally in this project as it is the higher energy isomer of HCO radical and may isomerize to the lower energy state.

The computational portion of this study reveals a higher number of $\mathrm{HCO} / \mathrm{HOC}$ radical formation pathways for the alcohol-substituted alkynes and focuses on the reactions of $\mathrm{O}\left({ }^{3} \mathrm{P}\right)+$ propargyl alcohol and $\mathrm{O}\left({ }^{3} \mathrm{P}\right)+3$-butyn-1-ol. The experimental portion of this study involves the detection of HCO radical as it forms during alkyne combustion via Cavity Ring-down Laser Absorption Spectroscopy (CRDLAS).

KEYWORDS: combustion; alkyne; formyl; radical; experimental; computational 


\title{
ALKYNE COMBUSTION: EXPERIMENTAL AND COMPUTATIONAL STUDIES OF FORMYL RADICAL PRODUCTION
}

\author{
MATTHEW C. DRUMMER
}

\author{
A Thesis Submitted in Partial \\ Fulfillment of the Requirements \\ For the Degree of \\ MASTER OF SCIENCE \\ Department of Chemistry

\section{ILLINOIS STATE UNIVERSITY}


(C)2018 Matthew C. Drummer 


\section{ALKYNE COMBUSTION: EXPERIMENTAL AND COMPUTATIONAL STUDIES OF FORMYL RADICAL PRODUCTION}

MATTHEW C. DRUMMER

COMMITTEE MEMBERS:

Jean M. Standard, Chair

Isabel X. Green

Christopher C. Mulligan 


\section{ACKNOWLEDGMENTS}

I would like to thank all of those who made this thesis possible. First, thank you to Dr. Jean Standard for taking me in as a graduate student and being an amazing research mentor and teacher of both computational and quantum chemistry. Second, thank you to Dr. Bob Quandt for sparking my interest in physical chemistry research and teaching me vital laboratory skills during my undergraduate years. Third, thank you to the faculty and staff of the Department of Chemistry at Illinois State University for nurturing a strong sense of community and fellowship among both undergraduate and graduate students. Finally, many thanks to Lori and Gary Drummer for all of their support and providing me with the means to further my education and pursue a career in science.

M.C.D. 


\section{CONTENTS}

\section{Page}

ACKNOWLEDGMENTS

CONTENTS

ii

TABLES

iv

FIGURES

CHAPTER

$\begin{array}{ll}\text { I. INTRODUCTION } & 1\end{array}$

$\begin{array}{ll}\text { II. EXPERIMENTAL METHODS } & 11\end{array}$

$\begin{array}{ll}\text { III. COMPUTATIONAL METHODS } & 15\end{array}$

$\begin{array}{lll}\text { IV. } & \text { RESULTS } & 18\end{array}$

Experimental Detection of $\mathrm{HCO}$ in the Reaction of $\mathrm{O}\left({ }^{3} \mathrm{P}\right)+$

$\begin{array}{lr}\text { Propargyl Alcohol } & 18\end{array}$

Computational Results for $\mathrm{O}\left({ }^{3} \mathrm{P}\right)+$ Propargyl Alcohol 23

Geometries: $\mathrm{O}\left({ }^{3} \mathrm{P}\right)+$ Propargyl Alcohol 24

Potential Energy Profiles: $\mathrm{O}\left({ }^{3} \mathrm{P}\right)+$ Propargyl Alcohol 28

Minimum Energy Crossing Point 41

NRT Analysis: $\mathrm{O}\left({ }^{3} \mathrm{P}\right)+$ Propargyl Alcohol 43

Computational Results for $\mathrm{O}\left({ }^{3} \mathrm{P}\right)+3$-Butyn-1-ol 53

Geometries: $\mathrm{O}\left({ }^{3} \mathrm{P}\right)+3$-Butyn-1-ol 53

Potential Energy Profiles: $O\left({ }^{3} \mathrm{P}\right)+3$-Butyn-1-ol 56

NRT Analysis: $\mathrm{O}\left({ }^{3} \mathrm{P}\right)+3$-Butyn-1-ol 62

$\begin{array}{ll}\text { Subsequent Pathways } & 69\end{array}$ 
$\begin{array}{ll}\text { V. CONCLUSION } & 72\end{array}$

$\begin{array}{ll}\text { REFERENCES } & 75\end{array}$

APPENDIX A: ALL OPTIMIZED STRUCTURES FOR O $\left({ }^{3} \mathrm{P}\right)+$ PROPARGYL

ALCOHOL AND O $\left({ }^{3} \mathrm{P}\right)+3-\mathrm{BUTYN}-1-\mathrm{OL}$ AT THE M06-2X/CC-PVTZ LEVEL

APPENDIX B: CARTESIAN COORDINATES FOR ALL OPTIMIZED

STRUCTURES FOR O $\left({ }^{3} \mathrm{P}\right)+$ PROPARGYL ALCOHOL AND O $\left({ }^{3} \mathrm{P}\right)+3-\mathrm{BUTYN}-1-\mathrm{OL}$

AT THE M06-2X/CC-PVTZ LEVEL

116 


\section{TABLES}

Table

Page

1. Selected bond lengths and angles for structures on the triplet potential energy surface of $\mathrm{O}\left({ }^{3} \mathrm{P}\right)+$ propargyl alcohol, calculated at the M06-2X/cc-pVTZ and $\mathrm{MP} 2 / 6-311++\mathrm{G}(\mathrm{d}, \mathrm{p})$ levels of theory.

2. Continued bond lengths and angles for structures on the triplet potential energy surface of $\mathrm{O}\left({ }^{3} \mathrm{P}\right)+$ propargyl alcohol, calculated at the M06-2X/cc-pVTZ and MP2/6-311++G(d,p) levels of theory.

3. Selected bond lengths and angles for structures on the singlet potential energy surface of $\mathrm{O}\left({ }^{3} \mathrm{P}\right)+$ propargyl alcohol, calculated at the M06-2X/cc-pVTZ and MP2/6-311++G(d,p) levels of theory.

4. Relative energies for structures on the triplet potential energy surface, computed using the M06-2X, MP2, and $\operatorname{CCSD}(\mathrm{T}) / / \mathrm{M} 06-2 \mathrm{X}$ single point methods.

5. Continued relative energies for structures on the triplet potential energy surface, computed using the M06-2X, MP2, and CCSD(T)//M06-2X single point methods.

6. Continued relative energies for structures on the triplet potential energy surface, computed using the M06-2X, MP2, and $\mathrm{CCSD}(\mathrm{T}) / / \mathrm{M} 06-2 \mathrm{X}$ single point methods.

7. MECP results, calculated at the M06-2X/cc-pVTZ level of theory.

8. Selected bond lengths and angles for structures on the triplet potential energy surface of $\mathrm{O}\left({ }^{3} \mathrm{P}\right)+3$-butyn-1-ol, calculated at the M06-2X/cc-pVTZ and MP2/6$311++\mathrm{G}(\mathrm{d}, \mathrm{p})$ levels of theory.

9. Continued selected bond lengths and angles for structures on the triplet potential energy surface of $\mathrm{O}\left({ }^{3} \mathrm{P}\right)+3$-butyn-1-ol, calculated at the M06-2X/cc-pVTZ and MP2/6-311++G(d,p) levels of theory. 


\section{FIGURES}

Figure

Page

1. Buettner's absorption spectrum of HCO (average of 10 runs) for the reaction of $\mathrm{O}\left({ }^{3} \mathrm{P}\right)+$ propyne.

2. Buettner's absorption spectrum of HCO (average of 10 runs) for the reaction of $\mathrm{O}\left({ }^{3} \mathrm{P}\right)+$ propargyl alcohol.

3. Relative HCO absorbance intensities for a series of alkynes.

4. Plot for level of theory vs basis set size. As accuracy increases, so does computational cost.

5. Potential energy profile for the reaction of $\mathrm{O}\left({ }^{3} \mathrm{P}\right)+$ propargyl alcohol at the MP2/6-311++G(d,p) level of theory.

6. Potential energy profile for the reaction of $\mathrm{O}\left({ }^{3} \mathrm{P}\right)+$ propyne at the MP2/6-311++G(d,p) level of theory (black = triplet, red = singlet).

7. Diagram of the CRDLAS experimental setup.

8. Example for 1 of 1536 data points from a single 613-617 nm scan (ring-down time: $\sim 15 \mu \mathrm{s})$.

9. Absorption spectrum for $\mathrm{HCO}$ in the reaction of $\mathrm{O}\left({ }^{3} \mathrm{P}\right)+$ propargyl alcohol. This spectrum is an average of 10 individual runs.

10. Absorption spectrum for $\mathrm{HCO}$ in the reaction of $\mathrm{O}\left({ }^{3} \mathrm{P}\right)+$ butyne. This spectrum is an average of 10 individual runs.

11. Absorption spectrum for $\mathrm{HCO}$ in the reaction of $\mathrm{O}\left({ }^{3} \mathrm{P}\right)+$ pentyne. This spectrum is an average of 10 individual runs.

12. Comparison of absorption spectra for $\mathrm{HCO}$ in the reaction of $\mathrm{O}\left({ }^{3} \mathrm{P}\right)+$ propyne (left) and $\mathrm{O}\left({ }^{3} \mathrm{P}\right)+$ propargyl alcohol (right). Left spectrum is an average of 15 individual runs and the right spectrum is an average of 8 individual runs.

13. Comparison of absorption spectra for $\mathrm{HCO}$ in the reaction of $\mathrm{O}\left({ }^{3} \mathrm{P}\right)+$ butyne (left) and $\mathrm{O}\left({ }^{3} \mathrm{P}\right)+3$-butyn-1-ol (right). Left spectrum is an average of 10 individual spectra and the right spectrum is an average of 10 individual runs.

14. Numbering scheme for the $\mathrm{O}\left({ }^{3} \mathrm{P}\right)+$ propargyl alcohol reaction.

15. Established triplet surface pathways, CCSD(T)// M06-2x/cc-pVTZ level, key IM and TS structures shown. 
16. Established singlet surface pathways, CCSD(T)//M06-2x/cc-pVTZ level.

17. Established triplet surface pathways, M06-2x/cc-pVTZ level.

18. Established singlet surface pathways, M06-2x/cc-pVTZ level.

19. Established triplet surface pathways, MP2/6-311++G(d,p) level.

20. Established singlet surface pathways, MP2/6-311++G(d,p) level.

21. Isomerization of the formyl radical, computed at the $\operatorname{CCSD}(\mathrm{T}) / / \mathrm{M} 06-2 \mathrm{x} / \mathrm{cc}-\mathrm{pVTZ}$ level of theory.

22. Novel HCO Pathway (branching off from IM-1T), CCSD(T)//M06-2x/cc-pVTZ level.

23. Novel HCO Pathway (branching off from IM-2T), CCSD(T)//M06-2x/cc-pVTZ level.

24. Novel HCO Pathway (branching off of IM-4T), CCSD(T)//M06-2x/cc-pVTZ level.

25. Structures for IM-1T (top left), MECP (top right), and IM-1S (bottom), calculated at the M06-2x/cc-pVTZ level.

26. NSD Data for the Established HCO Pathway, calculated at the M06-2x/cc-pVTZ level of theory.

27. NBO Data for the Established HCO Pathway, calculated at the M06-2x/cc-pVTZ level of theory.

28. NSD Data for a novel HCO Pathway (IM-1T), calculated at the M06-2x/cc-pVTZ level of theory.

29. NBO Data for a novel HCO Pathway (IM-1T), calculated at the M06-2x/cc-pVTZ level of theory.

30. NSD Data for a novel HOC Pathway (IM-2T), calculated at the M06-2x/cc-pVTZ level of theory.

31. NBO Data for a novel HOC Pathway (IM-2T), calculated at the M06-2x/cc-pVTZ level of theory. 
32. NSD Data for a novel HOC Pathway (IM-4T), calculated at the M06-2x/cc-pVTZ level of theory.

33. NBO Data for a novel HOC Pathway (IM-4T), calculated at the M06-2x/cc-pVTZ level of theory.

34. NSD Data for a novel HOC Pathway (IM-4T), calculated at the M06-2x/cc-pVTZ level of theory.

35. NBO Data for a novel HOC Pathway (IM-4T), calculated at the M06-2x/cc-pVTZ level of theory.

36. NSD Data for a novel HCO Pathway (IM-4T), calculated at the M06-2x/cc-pVTZ level of theory.

37. NBO Data for a novel HCO Pathway (IM-4T), calculated at the M06-2x/cc-pVTZ level of theory.

38. Numbering scheme for the $\mathrm{O}\left({ }^{3} \mathrm{P}\right)+3$-butyn-1-ol reaction.

39. Two HCO formation pathways for $\mathrm{O}\left({ }^{3} \mathrm{P}\right)+3$-butyn-1-ol, M06-2x/cc-pVTZ level.

40. Two HCO formation pathways for $\mathrm{O}\left({ }^{3} \mathrm{P}\right)+3$-butyn-1-ol, $\operatorname{CCSD}(\mathrm{T}) / / \mathrm{M} 06-2 \mathrm{x} /$ cc-pVTZ level.

41. Additional $\mathrm{HCO} / \mathrm{HOC}$ formation pathways for $\mathrm{O}\left({ }^{3} \mathrm{P}\right)+3$-butyn-1-ol, M06-2x/ cc-pVTZ level.

42. Additional $\mathrm{HCO} / \mathrm{HOC}$ formation pathways for $\mathrm{O}\left({ }^{3} \mathrm{P}\right)+3$-butyn-1-ol, $\mathrm{CCSD}(\mathrm{T}) / /$ M06-2x/cc-pVTZ level.

43. Final HOC dissociation pathway found for $\mathrm{O}\left({ }^{3} \mathrm{P}\right)+3$-butyn-1-ol, $\mathrm{M} 06-2 \mathrm{x} /$ cc-pVTZ level.

44. Final HOC dissociation pathway found for $\mathrm{O}\left({ }^{3} \mathrm{P}\right)+3$-butyn-1-ol, $\mathrm{CCSD}(\mathrm{T}) / /$ M06-2x/cc-pVTZ.

45. NSD Data for the HCO pathway shown in Figure 39, calculated at the M06-2x/ cc-pVTZ level of theory.

46. NBO Data for the HCO pathway shown in Figure 39, calculated at the M06-2x/ cc-pVTZ level of theory. 
47. NSD Data for the other HCO pathway shown in Figure 39, calculated at the M06-2x/ cc-pVTZ level of theory.

48. NBO Data for the other HCO pathway shown in Figure 39, calculated at the M06-2x/ cc-pVTZ level of theory.

49. NSD Data for the HCO pathway shown in Figure 41, calculated at the M06-2x/ cc-pVTZ level of theory.

50. NBO Data for the HCO pathway shown in Figure 41, calculated at the M06-2x/ cc-pVTZ level of theory.

51. NSD Data for the HOC pathway shown in Figure 41, calculated at the M06-2x/ cc-pVTZ level of theory.

52. NBO Data for the HOC pathway shown in Figure 41, calculated at the M06-2x/ cc-pVTZ level of theory.

53. NSD Data for the HOC pathway shown in Figure 43, calculated at the M06-2x/ cc-pVTZ level of theory.

54. NBO Data for the HOC pathway shown in Figure 43, calculated at the M06-2x/ cc-pVTZ level of theory.

55. Subsequent dissociation pathway for HOC, $\operatorname{CCSD(T)//M06-2x/cc-pVTZ~level.~}$

56. Subsequent dissociation pathway for HOC, CCSD(T)//M06-2x/cc-pVTZ level.

57. Subsequent dissociation pathway for $\mathrm{HCO}, \mathrm{CCSD}(\mathrm{T}) / / \mathrm{M} 06-2 \mathrm{x} / \mathrm{cc}-\mathrm{pVTZ}$ level. 


\section{CHAPTER I}

\section{INTRODUCTION}

The combustion of hydrocarbons has been a subject of interest to scientists for a whole host of reasons, including both its contributions to human advancement and its potential environmental consequences. The industrial-scale process of burning fossil fuels such as petroleum, coal, and natural gas allows for large yields in energy that is put to work within various sectors including transportation, residential/commercial energy utilization, and the generation of electrical power. ${ }^{1}$ The U.S. Energy Information Administration (EIA) estimates that a total of 97,394 trillion BTUs (British Thermal Unit, $1 \mathrm{BTU}=1.055 \mathrm{~kJ}$ ) of energy were consumed by the United States alone in 2016, with the current total estimated consumption of 2017 at 97,310 trillion BTUs. ${ }^{2}$ With fossil fuel combustion making up over $80 \%$ of total energy consumption in the United States, ${ }^{2}$ the effects of chemical byproducts from these combustion reactions, therefore, come into question. Perhaps the most profound effect of combustion byproducts is the phenomenon of global climate change in which human activities of energy consumption have led to the highest levels of atmospheric $\mathrm{CO}_{2}$ in 650,000 years (404.07 ppm), increased global temperature of $1.7^{\circ} \mathrm{F}$, increased loss of land ice (281.0 gigatonnes per year), and a rise in sea level of about seven inches over the past 100 years. $^{3}$

Scientists are interested in studying the reaction mechanisms of hydrocarbon combustion in an effort to gain further insight into how combustion reactions work and the intermediate byproducts that are inevitably released into the atmosphere. Scientific research into the mechanisms and effects of hydrocarbon combustion spans a number of fields, including physical chemistry, and such mechansisms have been studied experimentally and computationally by physical chemists for over two decades. ${ }^{4-6}$ Through experimentation and computational methods, 
physical chemists have detected key reaction pathways that have been proven to exist throughout many hundreds of saturated hydrocarbon combustion reactions. However, the combustion of the unsaturated analogs of these hydrocarbons remains widely uninvestigated. Investigations of the combustion of unsaturated hydrocarbons have mostly been focused on short-chain alkenes and alkynes. Short-chain alkenes that have been studied extensively include ethylene $\mathrm{e}^{7-9}$ and propene. ${ }^{10-12}$ Investigations on the combustion of alkynes have been conducted mainly on acetylene ${ }^{13-17}$ and propyne. ${ }^{18-20}$ Research on the reactions of $\mathrm{O}\left({ }^{3} \mathrm{P}\right)+$ short-chain alkenes have been carried out by the Cvetanović group, in which temperature dependence was observed for the combustion of ethylene, propene, and 1-butene. ${ }^{21}$ In addition, the Cvetanović group determined that radical intermediates are produced during these combustion reactions before the final products, $\mathrm{CO}_{2}$ and $\mathrm{H}_{2} \mathrm{O}$, are formed. ${ }^{21}$ The high temperature conditions of a combustion reaction allow for the dehydrogenation of saturated hydrocarbons to their corresponding unsaturated forms, as described by equations (1) and (2). ${ }^{22}$ Following the dissociation of molecular oxygen, equation (3), alkene and alkyne species may react with $\mathrm{O}\left({ }^{3} \mathrm{P}\right)$ atoms to form many products, as shown in equations (4) and (5),

$$
\begin{aligned}
& \text { alkane }+ \text { heat } \rightarrow \text { alkene }+2 \mathrm{H} \\
& \text { alkene }+ \text { heat } \rightarrow \text { alkyne }+2 \mathrm{H} \\
& \mathrm{O}_{2}+\text { heat } \rightarrow 2 \mathrm{O}\left({ }^{3} \mathrm{P}\right) \\
& \text { alkene }+\mathrm{O}\left({ }^{3} \mathrm{P}\right) \rightarrow \text { products } \\
& \text { alkyne }+\mathrm{O}\left({ }^{3} \mathrm{P}\right) \rightarrow \text { products }
\end{aligned}
$$


Numerous product channels exist in an alkyne $+\mathrm{O}\left({ }^{3} \mathrm{P}\right)$ reaction, including $\mathrm{CO}, \mathrm{H}_{2}$, and various radical species. Reaction pathways of alkenes and alkynes with $\mathrm{O}\left({ }^{3} \mathrm{P}\right)$ have been studied by several research groups and a number of recurring reaction pathways have been discovered, including the formation of the formyl radical, $\mathrm{HCO} .{ }^{23}$ The formyl radical, $\mathrm{HCO}$, was first reported by Vaidya in 1934 and has since been proven to be an abundantly observed intermediate in many hundreds of combustion reactions. ${ }^{24}$ For example, research conducted by the Bersohn group delved into the $\mathrm{HCO}$ radical formation pathways within the $\mathrm{O}\left({ }^{3} \mathrm{P}\right)+$ alkene systems. ${ }^{25}$ The formyl radical is a highly reactive atmospheric compound that may react and result in the formation of hydroxyl radicals, $\mathrm{OH}$, a species that reacts with other atmospheric compounds to form aerosol particles and acid rain. ${ }^{22} \mathrm{HCO}$ radical plays significant roles in combustion, atmospheric, and interstellar chemical reactions, particularly as a donor of hydrogen atoms. ${ }^{26}$ Experimental $\mathrm{HCO}$ radical detection in $\mathrm{O}\left({ }^{3} \mathrm{P}\right)+$ alkyne reactions remains largely uninvestigated due to the complications of detecting HCO radical via laser-induced fluorescence (LIF), a widely utilized gas-phase spectroscopic method. ${ }^{27}$ The complications of employing LIF to study HCO radical formation stems from an interference of the $\mathrm{O}\left({ }^{3} \mathrm{P}\right)$ precursor, $\mathrm{SO}_{2}$, which absorbs in the same region as the $\mathrm{B} \leftarrow \mathrm{X}$ transition of $\mathrm{HCO}$ radical observed in LIF $(\sim 230 \mathrm{~nm})$. Cavity ringdown laser absorption spectroscopy (CRDLAS) allows one to observe the $\tilde{A}^{2} A^{\prime \prime}(0,9,0) \leftarrow \tilde{X}$ ${ }^{2} A^{\prime}(0,0,0)$ transition of $\mathrm{HCO}$ radical $(613-617 \mathrm{~nm})$ without any interference from $\mathrm{SO}_{2}$.

The Quandt group has conducted CRDLAS experiments to demonstrate the formation of HCO radical within the reactions of $\mathrm{O}\left({ }^{3} \mathrm{P}\right)$ with propyne, butyne, 3-butyn-2-one, 3-butyn-1-ol, pentyne, propargyl ether, propargyl amine, and propargyl alcohol.$^{28}$ These studies revealed wide variations in $\mathrm{HCO}$ radical absorption intensity across the different $\mathrm{O}\left({ }^{3} \mathrm{P}\right)+$ alkyne reactions. For example, absorption spectra collected by the Quandt group ${ }^{28}$ for propyne and propargyl alcohol 
are shown in Figures 1 and 2, respectively. It was observed that the reaction of $\mathrm{O}\left({ }^{3} \mathrm{P}\right)$ with propyne and butyne resulted in detectable $\mathrm{HCO}$ radical absorption, whereas $\mathrm{O}\left({ }^{3} \mathrm{P}\right)+$ pentyne resulted in data that was far too noisy to distinguish HCO radical formation. Propargyl alcohol consistently gave the strongest intensity signal for $\mathrm{HCO}$ radical.

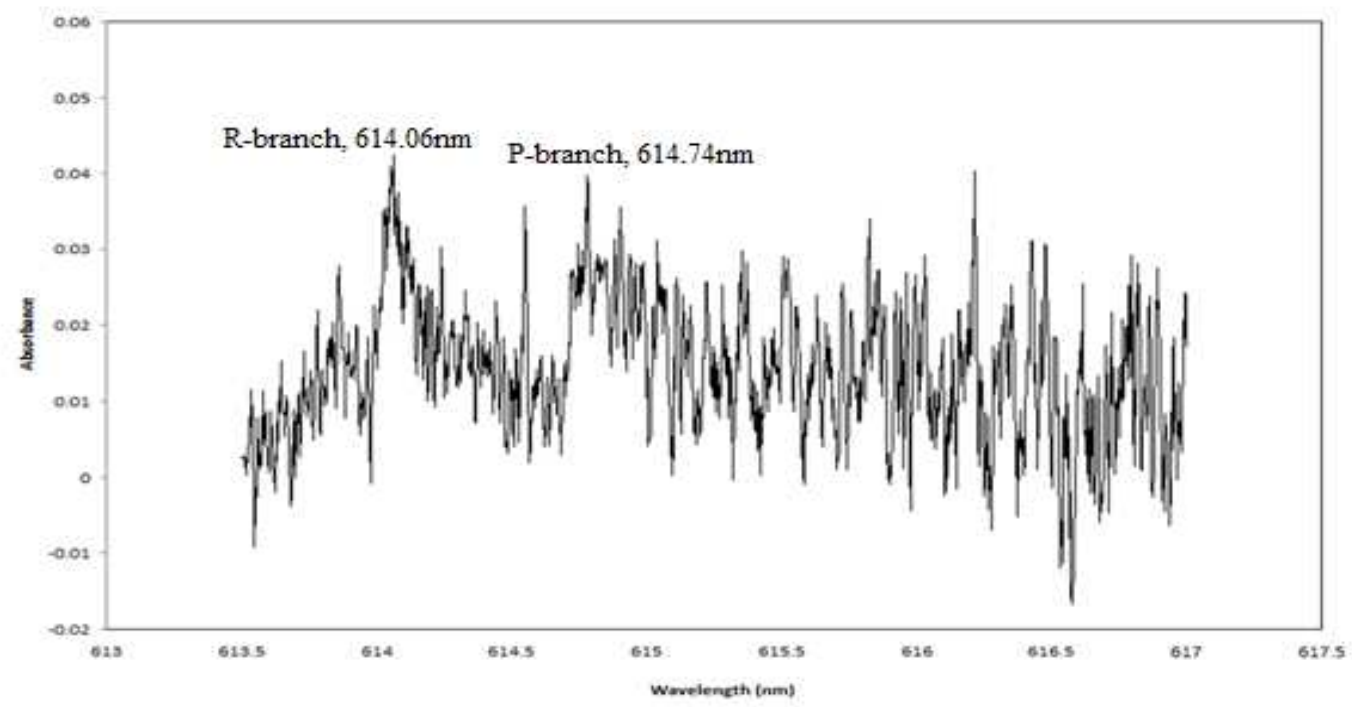

Figure 1. Buettner's absorption spectrum of $\mathrm{HCO}$ (average of 10 runs) for the reaction of $\mathrm{O}\left({ }^{3} \mathrm{P}\right)+$ propyne. ${ }^{28}$

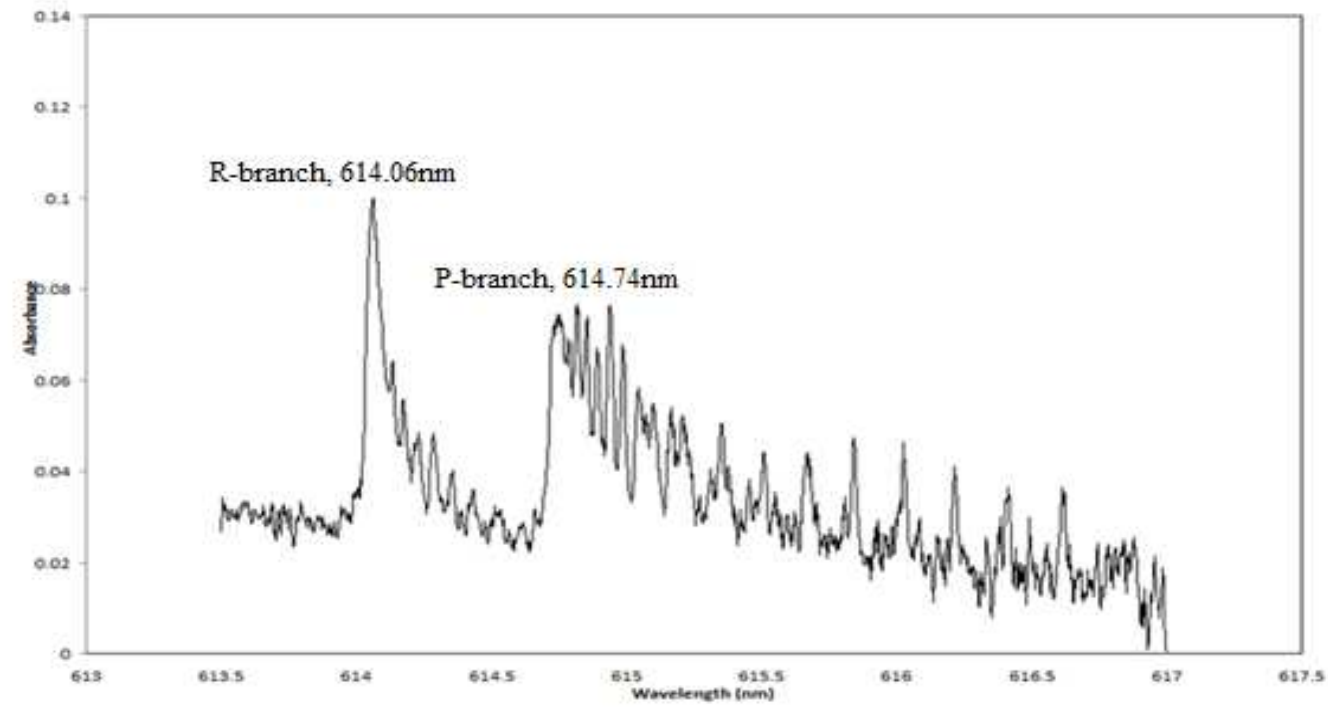

Figure 2. Buettner's absorption spectrum of $\mathrm{HCO}$ (average of 10 runs) for the reaction of $\mathrm{O}\left({ }^{3} \mathrm{P}\right)+$ propargyl alcohol. ${ }^{28}$ 
Previous experimental work in the Quandt research lab found a relatively large signal in the absorption spectrum for $\mathrm{HCO}$ radical formation in propargyl alcohol $+\mathrm{O}\left({ }^{3} \mathrm{P}\right) \cdot{ }^{28}$ Formyl radical signals were acquired for a series of other alkynes as well, with their relative absorbance intensities compared in Figure 3. Absorbance values below 0.02 have been covered by a dark area to represent where the signal becomes indistinguishable from the noise. Prior to the studies conducted by the Quandt and Standard lab groups, the reaction pathway of propargyl alcohol has gone uninvestigated and, thus, has become a topic of interest as hydroxyl-substituted alkynes gave the strongest absorbance signal for $\mathrm{HCO}$ radical after reacting with triplet oxygen.

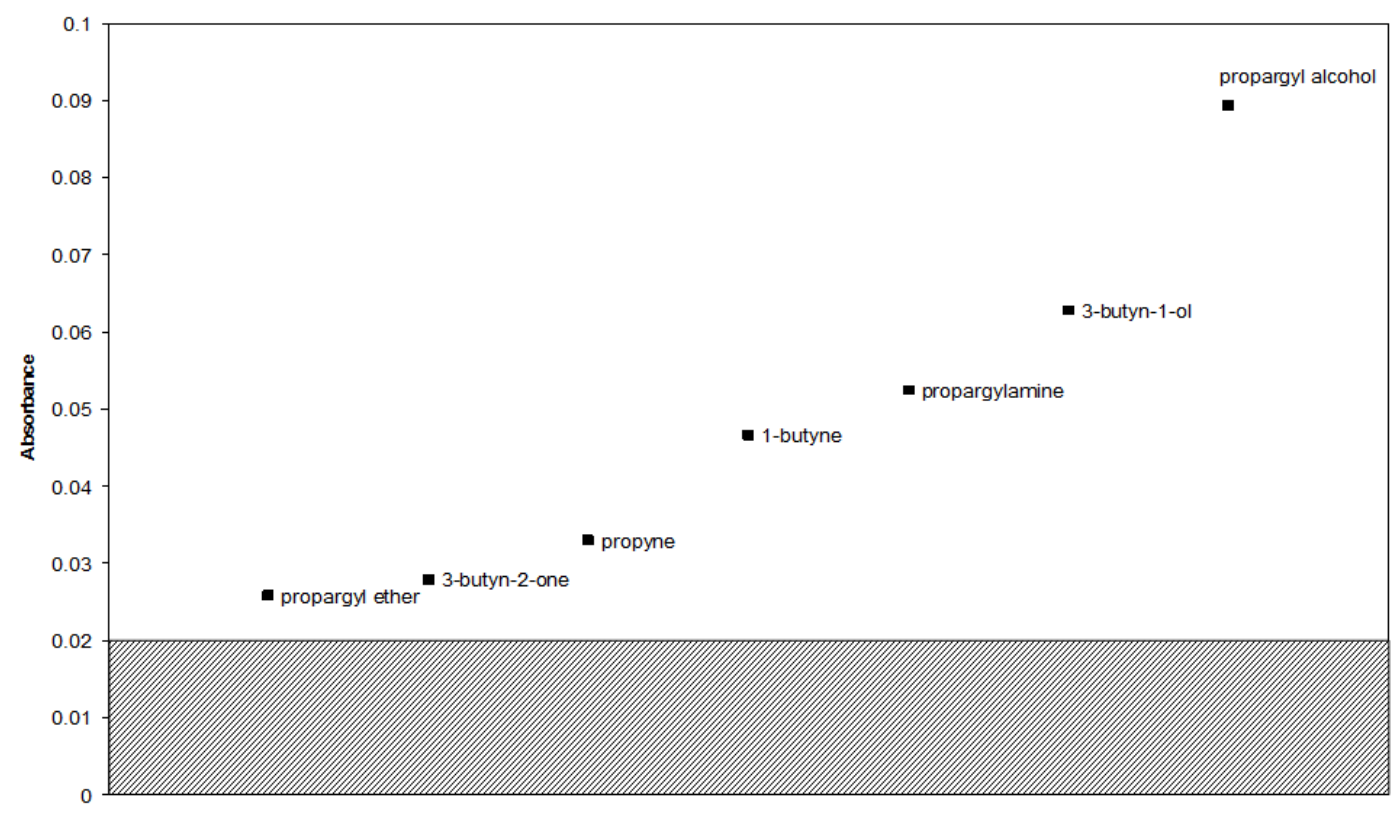

Figure 3. Relative HCO absorbance intensities for a series of alkynes. ${ }^{28}$

Through computational methods, the Quandt and Standard research groups studied the potential energy surfaces of $\mathrm{O}\left({ }^{3} \mathrm{P}\right)+$ alkyne reactions to better understand the reaction mechanisms behind formyl radical formation. ${ }^{28,29}$ Previous computational work in the Quandt and Standard groups have led to new insight into how HCO radical forms during combustion 
reactions. ${ }^{28-29}$ Computational methods have been employed to investigate novel reaction pathways that may explain the strong $\mathrm{HCO}$ radical signal seen in the propargyl alcohol $+\mathrm{O}\left({ }^{3} \mathrm{P}\right)$ reaction. The Quandt and Standard groups utilized quantum mechanical calculations for the optimization of equilibrium geometries, vibrational frequencies, and potential energies at the B3LYP/cc-pVTZ, M06-2x/cc-pVTZ, and MP2/6-311++G(d,p) levels of theory. More accurate potential energy values were obtained by performing single-point energy calculations on each optimized structure at the CCSD(T)/cc-pVTZ//M06-2x/cc-pVTZ level of theory.

Figure 4 illustrates the relative accuracy and computational cost of different levels of theory and basis set sizes. The concurrent utilization of high levels of theory with large basis set sizes results in highly accurate optimization results. However, computational costs increase with accuracy. For this reason, the $\operatorname{CCSD}(\mathrm{T})$ level of theory was not feasibly applicable for full optimizations of the various reagent, IM, TS, and product structures for this project. Instead, methods with lower computational costs were employed for optimizing geometries and vibrational frequencies. Once this has been accomplished, the higher level CCSD(T) was used to optimize only the potential energies of each structure. 


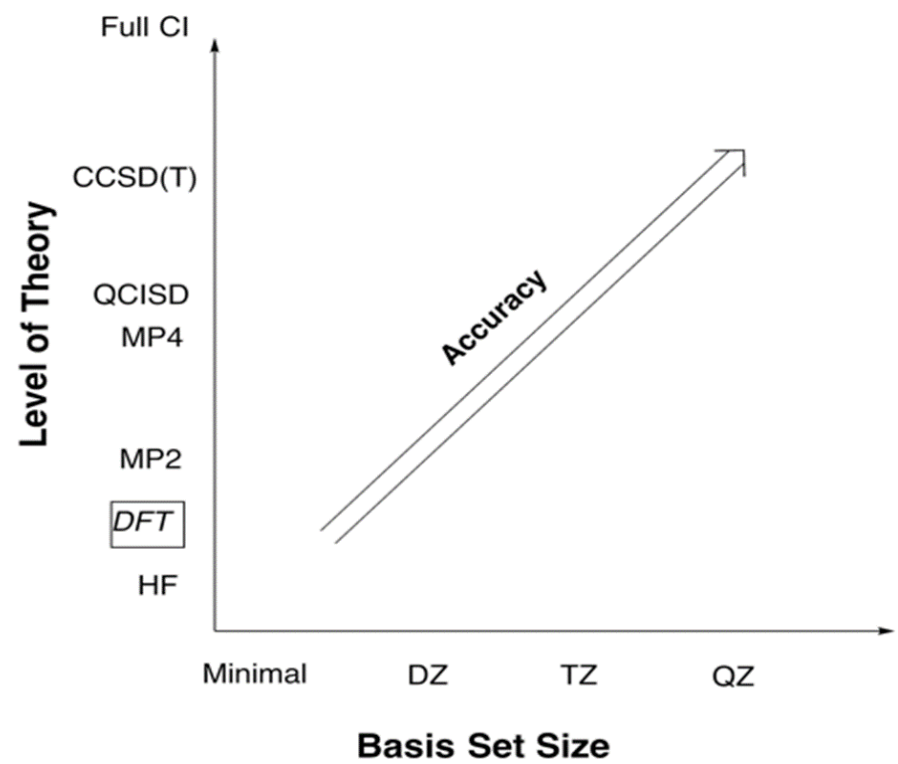

Figure 4. Plot for level of theory vs basis set size. As accuracy increases, so does computational cost.

Thus far, only a few pathways in the reaction of $\mathrm{O}\left({ }^{3} \mathrm{P}\right)+$ propargyl alcohol have been studied and were investigated at the MP2/6-311++G(d,p) level of theory, as seen in Figure 5. The pathways shown in Figure 5 exist on the triplet potential energy surface of the $\mathrm{O}\left({ }^{3} \mathrm{P}\right)+$ propargyl alcohol reaction. The relative energies of all intermediate (IM) and transition state (TS) structures in Figure 5 have been improved upon and are presented in this project. Optimization of potential energies was achieved by first collecting optimized geometries, vibrational frequencies, and energies at the M06-2x level of theory followed by single-point energy calculations at the $\operatorname{CCSD}(\mathrm{T}) / \mathrm{cc}-\mathrm{pVTZ}$ level of theory. 


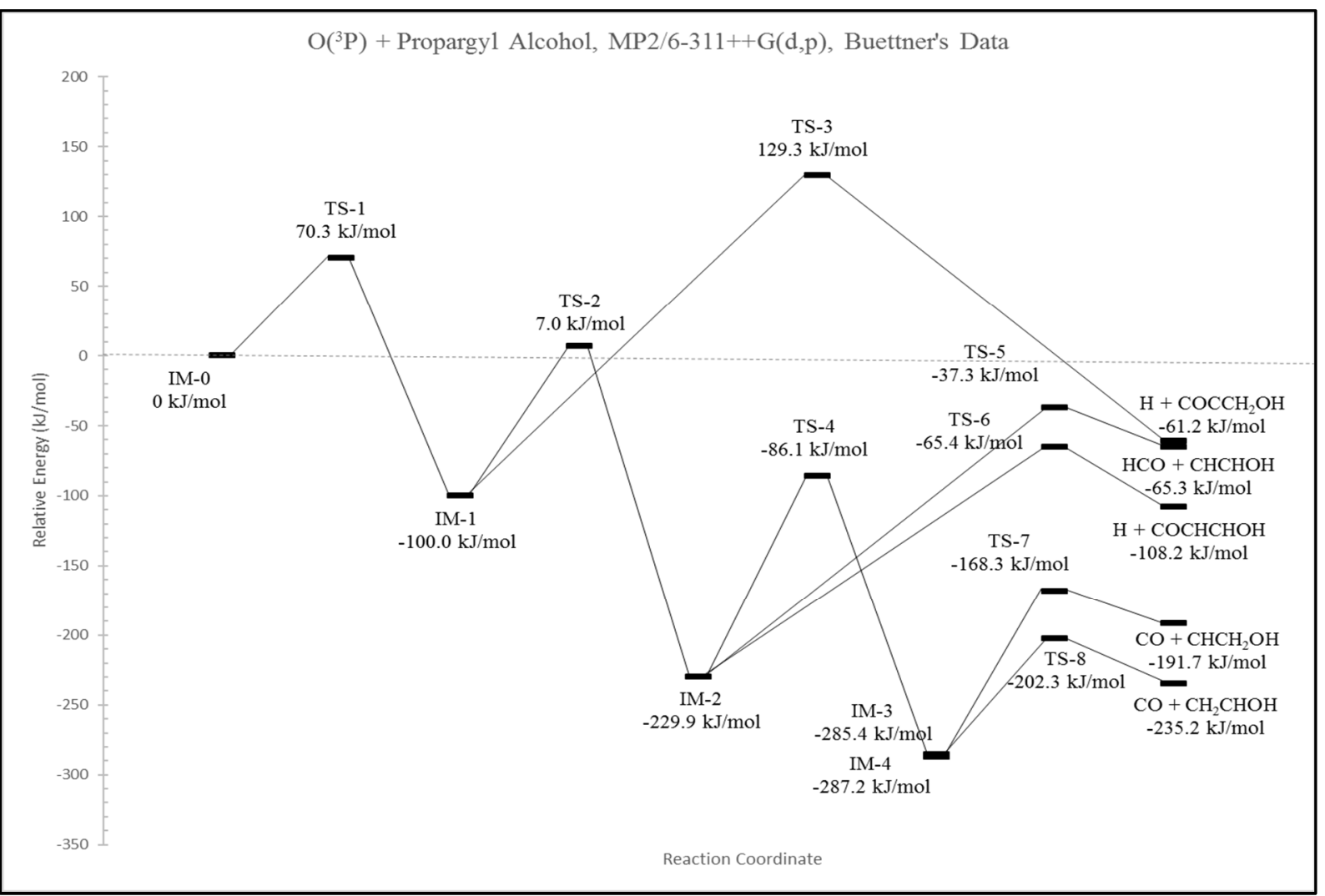

Figure 5. Potential energy profile for the reaction of $\mathrm{O}\left({ }^{3} \mathrm{P}\right)+$ propargyl alcohol at the MP2/6$311++\mathrm{G}(\mathrm{d}, \mathrm{p})$ level of theory. ${ }^{28}$

As seen in Figure 6, both the triplet (black) and singlet (red) pathways were computed by the Quandt and Standard groups for the $\mathrm{O}\left({ }^{3} \mathrm{P}\right)+$ propyne reaction. While the singlet surface pathways do not yield HCO radical, intersystem crossing (ISC) may occur in which a triplet IM may cross over to the singlet surface or vice versa. ISC plays an important role in many atmospheric and combustion reactions as it helps explain the branching ratios and intersections of product channels in the reactions of $\mathrm{O}\left({ }^{3} \mathrm{P}\right)+$ alkanes, alkenes, and alkynes. ${ }^{30}$ ISC between the singlet and triplet surfaces is of interest because of the accessibility of the singlet potential energy surface after the initiation of the reaction on the triplet surface. 


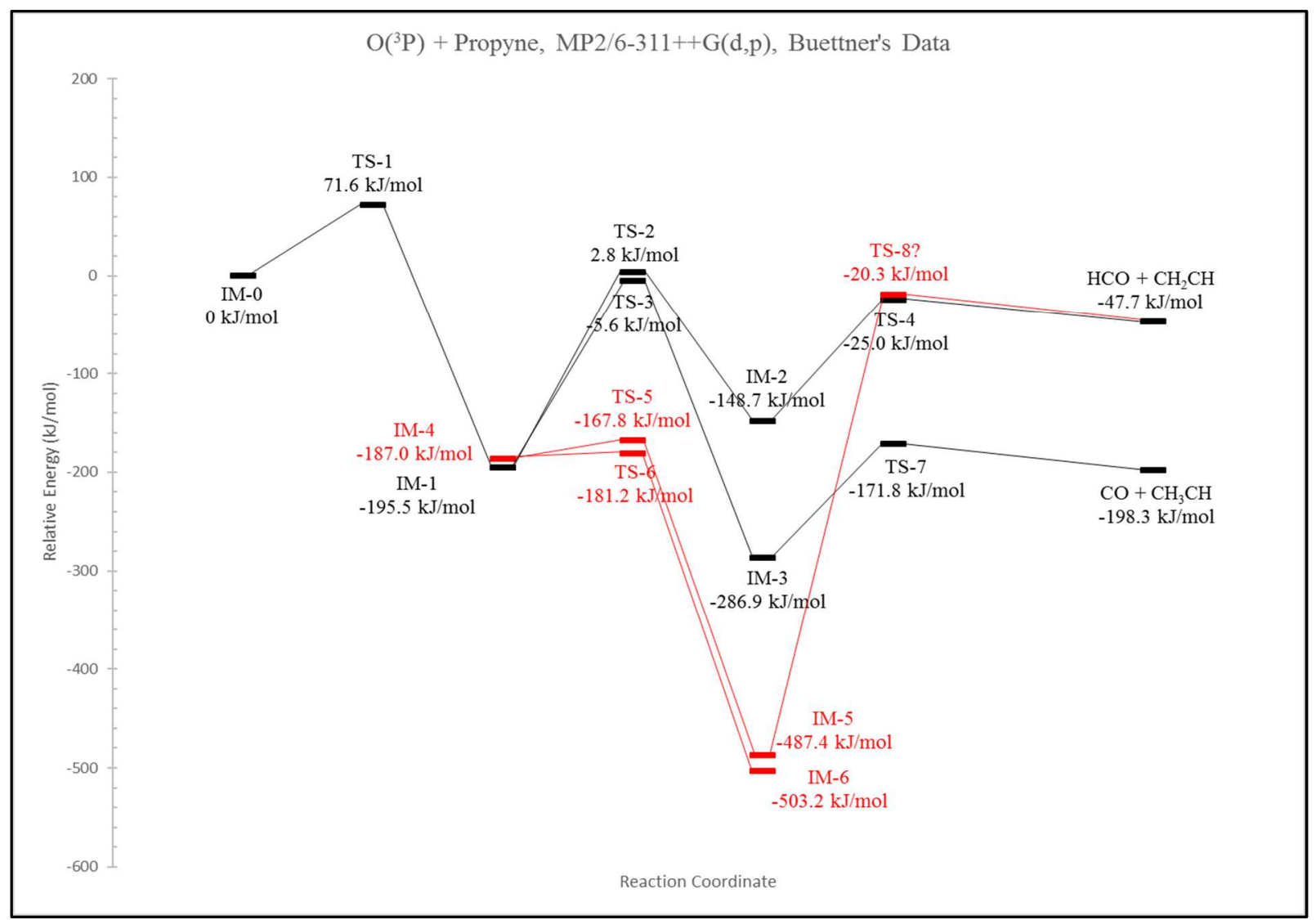

Figure 6. Potential energy profile for the reaction of $\mathrm{O}\left({ }^{3} \mathrm{P}\right)+$ propyne at the $\mathrm{MP} 2 / 6-311++\mathrm{G}(\mathrm{d}, \mathrm{p})$ level of theory $($ black $=$ triplet, red $=$ singlet $){ }^{28}$

The potential energy profile (PEP) is a plot of potential energy as a function of reaction coordinate. As the reaction progresses, IM and TS structures reveal important information about the combustion reaction of interest, such as relative energy barriers that indicate which reaction pathways are preferred. Calculated vibrational frequencies confirm whether a structure is an IM (all vibrational frequencies are positive) or a TS (one vibrational frequency is imaginary).

Buettner explored the reaction pathways for the reactions of $\mathrm{O}\left({ }^{3} \mathrm{P}\right)+$ propyne, butyne, pentyne, and propargyl alcohol at the MP2 level of theory. ${ }^{28}$ This project expands upon Buettner's findings and offers possible explanations as to why propargyl alcohol gave the highest intensity absorption signal for HCO radical in the CRDLAS studies. 
The first accomplishment presented in this thesis is the rebuilt PEP of $\mathrm{O}\left({ }^{3} \mathrm{P}\right)+$ propargyl alcohol at the $\operatorname{CCSD}(\mathrm{T}) / \mathrm{cc}-\mathrm{pVTZ} / \mathrm{M} 06-2 \mathrm{x} / \mathrm{cc}-\mathrm{pVTZ}$ level of theory because M06-2x has been proven to yield optimized geometries and energies that are more consistent with $\operatorname{CCSD}(\mathrm{T})$ for the reaction of propyne $+\mathrm{O}\left({ }^{3} \mathrm{P}\right)$ than other benchmarked levels of theory. ${ }^{29}$ The HCO radical formation pathway found by Buettner will hereby be referred to as the "established HCO radical pathway." In addition to building a new PEP starting from Buettner's MP2/6-311++G(d,p) structures, novel pathways of formyl radical production have also been investigated. All novel pathways presented here branch off from IM structures within the established HCO radical formation pathway. The formation of the isoformyl radical, $\mathrm{HOC}$, is also investigated and presented here as it isomerizes to the lower energy formyl radical, HCO. Reaction mechanisms of $\mathrm{HCO}$ and $\mathrm{HOC}$ radical production are investigated and presented in this thesis via Natural Resonance Theory (NRT) analysis, from which bond orders and spin densities are obtained. Finally, this project is concluded with the study of the reaction of $\mathrm{O}\left({ }^{3} \mathrm{P}\right)+3$-butyn-1-ol. Full equilibrium geometry, vibrational frequency, and energy optimizations have been carried out at the M06-2x/cc-pVTZ level of theory with single-point energy calculations collected at the $\operatorname{CCSD}(\mathrm{T}) / \mathrm{cc}-\mathrm{pVTZ}$ level. Both the $\mathrm{O}\left({ }^{3} \mathrm{P}\right)+$ propargyl alcohol and $\mathrm{O}\left({ }^{3} \mathrm{P}\right)+3$-butyn-1-ol reactions have received a full NRT analysis, which is presented in chapter IV. 


\section{CHAPTER II}

\section{EXPERIMENTAL METHODS}

The experimental portion of this project was completed in the Quandt research group. HCO radical product formation was detected via Cavity Ringdown Laser Absorption Spectroscopy (CRDLAS). ${ }^{28}$ The experimental set up, as seen in Figure 7, involved flowing reagent gaseous species through a stainless steel cavity consisting of two $27 \mathrm{~cm}$ tubes connected to an $8 \mathrm{~cm}$ stainless steel cubic cell. Reagents were introduced into the cavity by flowing the appropriate gas/vapor through small tubes connected to the system and were evacuated with a vacuum pump (base pressure of $\sim 1$ torr). Each end of the cavity was sealed off from the atmosphere by highly reflective mirrors (Los Gatos Research, R>99.999\%).

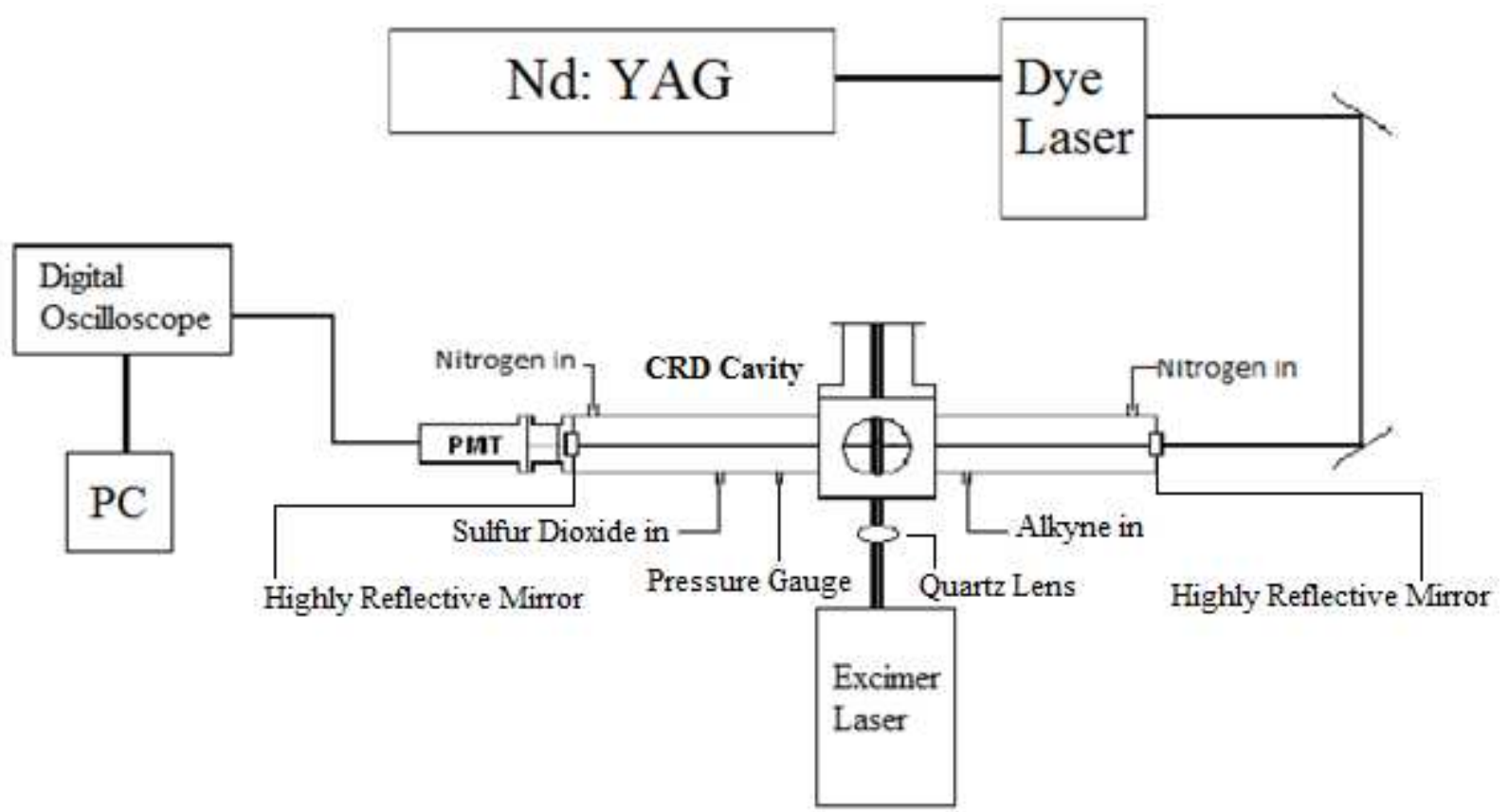

Figure 7. Diagram of the CRDLAS experimental setup. 
Reactions were probed using a Nd:YAG laser (Continuum Surelite II, operating at 1064 $\mathrm{nm}$ ) which passed through a tunable pumped dye laser (Continuum ND6000). The YAG/dye laser setup fired at a frequency of $2 \mathrm{~Hz}$ and at a power of 5-10 mJ. The dye used for the dye pump was rhodamine 640 , providing a wavelength range of $605-630 \mathrm{~nm}$ with a peak wavelength of $613 \mathrm{~nm}$. One end of the stainless steel cavity featured a photomultiplier tube (PMT) for detection of light decay. This detector was then connected to a digital oscilloscope (PicoScope 2207A, Pico Technologies) which was connected to a PC with a USB adapter to record the ringdown data. Typical ringdown times ranged from 30-70 $\mu$ s.

CRDLAS is a modification of a technique known as Cavity Attenuated Phase Shift (CAPS), which was developed initially to quantify the reflectivity of mirrors. CAPS uses continuous wave laser light to measure the phase shift between the input and output light in the cavity. ${ }^{31}$ However, this technique was limited in its sensitivity due to fluctuations in the phase angle. CRDLAS differs from CAPS in that the modulated light entering the cavity is pulsed, as opposed to continuous. Instead of measuring the phase shift angle, CRDLAS relies on the time it takes for the light intensity to decay after a given pulse. The intensity decay observed in a CRDLAS experiment can be expressed as a first-order relationship between the initial intensity of modulated light and intensity decay as a function of time, equation (6).

$$
I(t)=I_{0} e^{-t / \tau}
$$

Here, $I(t)$ is the intensity at time $t, I_{0}$ is the initial intensity, and $\tau$ is the ringdown time. The ringdown time, $\tau$, is defined as the amount of time it takes for the light to exponentially decay inside the cavity and is hastened by the presence of a chemical species that absorbs light at 
a wavelength that is within the scan-range of the spectrometer. According to the Beer-Lambert Law, absorption $(A)$ equals the concentration of analyte $(c)$ times the molar absorptivity $(\varepsilon)$ and path length $(l)$, equation (7).

$$
A=\varepsilon l c
$$

CRDLAS allows for the detection of minute product concentrations ( $\sim 10 \mathrm{ppb})$ by compensating with a large path length. The highly reflective mirrors allow the probe light to reflect back and forth within the cavity, resulting in an effective path length of $\sim 20 \mathrm{~km}$.

The generation of $\mathrm{O}\left({ }^{3} \mathrm{P}\right)$ atoms was achieved via photolysis of $\mathrm{SO}_{2}$ at $193 \mathrm{~nm}$, shown in equation (8), using an Ar/F Excimer Laser (Lambda Physik Compex 110), operating at a frequency of $2 \mathrm{~Hz}$ and a power of 94-114 mJ.

$$
\mathrm{SO}_{2}+h v(193 \mathrm{~nm}) \rightarrow \mathrm{SO}\left(A^{3} \Pi\right)+O\left({ }^{3} \mathrm{P}\right)
$$

The scan range chosen for probing HCO radical was 613-617 nm. HCO radical also shows absorption in the UV range at $230 \mathrm{~nm}$ for its ground to second excited state transition (B $\leftarrow \mathrm{X})$, however, the precursor for $\mathrm{O}\left({ }^{3} \mathrm{P}\right)\left(\mathrm{SO}_{2}\right)$ absorbs strongly in the UV region and so 613-617 $\mathrm{nm}$ was chosen as the region to look for $\mathrm{HCO}$ radical formation as the P and R bands (band origins at $614.4 \mathrm{~nm}$ and $613.8 \mathrm{~nm}$, respectively) are found in that region for the formyl radical $\tilde{A}^{2} A^{\prime \prime}(0,9,0) \leftarrow \tilde{X}^{2} A^{\prime}(0,0,0)$ transition.

Nitrogen gas was flowed through the cavity in addition to $\mathrm{SO}_{2}$ and the alkyne of interest. The $\mathrm{N}_{2}$ acted as a vibrational relaxant for $\mathrm{HCO}$ radical as well as a barrier to protect the mirrors 
against build-up of contaminants during an experiment. Typical pressures were about 4 torr with a reagent gas ratio of 1:1:2 ( $\mathrm{N}_{2}: \mathrm{SO}_{2}$ :alkyne) and were monitored using a digital barometer. The statistical program language, $\mathrm{R}$, was utilized to process the ringdown time data (exponential decay time $v s$. wavelength) into an absorption spectrum (absorption $v s$. wavelength). ${ }^{32}$ Experimental studies were conducted on propyne, butyne, pentyne, and propargyl alcohol (Aldrich, 99\%). CRDLAS results are presented and discussed in chapter IV. 


\section{CHAPTER III}

\section{COMPUTATIONAL METHODS}

Quantum mechanical methods were employed to gain insight into the mechanisms at play within the alkyne $+\mathrm{O}\left({ }^{3} \mathrm{P}\right)$ reactions of interest. Previous work by Buettner ${ }^{28}$ revealed varying degrees of signal intensity for the absorption spectrum of HCO radical without plausible explanation for the observed intensity variation across alkyne species. Computational methods were utilized to search for reaction pathways to help explain the higher intensity HCO radical absorption observed for the reaction of $\mathrm{O}\left({ }^{3} \mathrm{P}\right)$ with hydroxyl-substituted alkynes (propargyl alcohol and 3-butyn-1-ol). Both the triplet and singlet potential energy surfaces of the $\mathrm{O}\left({ }^{3} \mathrm{P}\right)+$ propargyl alcohol and $\mathrm{O}\left({ }^{3} \mathrm{P}\right)+3$-butyn-1-ol reactions were investigated and are presented here. While formyl radical production is only observed on the triplet potential energy surface of these reactions, the singlet surface is easily accessible via ISC and is, thus, relevant to this study.

Theoretical investigations were carried out using the Gaussian 09 software package. ${ }^{33}$ Calculations were performed at the M06-2x/cc-pVTZ level of theory, a DFT-based functional that has proven to yield energy values comparable to higher levels of theory without the computational cost. ${ }^{29}$ Previous work in the Quandt and Standard groups have employed the MP2(Full) method with a 6-311++G(d,p) basis set for geometry optimizations. ${ }^{28,29}$ When MP2 potential energy values were compared to $\operatorname{CCSD}(\mathrm{T})$ single-point energy values, it was observed that MP2 consistently predicted higher energy barriers due to the shorter estimation of bond lengths. To acquire more reliable geometries with low resource cost, the M06-2x functional was adopted as it yielded potential energy values that were more comparable to the values found at the highly reliable $\operatorname{CCSD}(\mathrm{T}) / \mathrm{cc}-\mathrm{pVTZ}$ level. The computational methods discussed here have been performed for the reactions of $\mathrm{O}\left({ }^{3} \mathrm{P}\right)$ with propargyl alcohol and 3-butyn-1-ol. 
Once optimized geometries, vibrational frequencies, and potential energies are obtained at the M06-2x/cc-pVTZ level of theory, a higher level of theory may be employed to obtain more accurate potential energy values. The coupled cluster method, $\operatorname{CSD}(\mathrm{T}) / \mathrm{cc}-\mathrm{pVTZ}$, was utilized to conduct single-point energy calculations on the optimized M06-2x/cc-pVTZ structures.

The collected vibrational frequency data was utilized to identify structures as either intermediates (IM) or transition states (TS). TS structures exist at the saddle point of the potential energy surface of a reaction and have imaginary values for the frequency that describes the transition, such as a hydrogen shift or a dissociation. IM structures exist at local minima on the potential energy surface and have all positive, real values for their vibrational frequencies. Intrinsic reaction coordinate (IRC) calculations were carried out on most TS structures to verify that a given TS connected two IM structures along the reaction coordinate. The output file for an IRC may show 100 or more structures that connect a TS structure to its corresponding precursor and product IM structures.

Minimum energy crossing point (MECP) calculations have also been performed for the propargyl alcohol/3-butyn-1-ol $+\mathrm{O}\left({ }^{3} \mathrm{P}\right)$ reactions to reveal where intersystem crossing (ISC) occurs within the reaction pathway. ${ }^{34,35}$ ISC is the phenomenon by which a reaction crosses from the triplet surface to the singlet surface or vice versa. Typically, ISC can be thought of as a seam on the three dimensional potential energy surface of the reaction. The MECP calculation will find the lowest energy point on the ISC seam and provides the point within the reaction pathway and the geometric parameters for which crossing may occur.

Finally, a Natural Resonance Theory (NRT) analysis was carried out on the reactions discussed in this project. NRT is a method developed by Frank Weinhold of University of Wisconsin-Madison ${ }^{36-40}$ and provides two key pieces of information that are crucial for 
understanding the reaction mechanisms at play: natural spin density (NSD) and natural bond order (NBO). NSD reveals the movement of the unpaired electrons on the triplet potential energy surface as the reaction progresses and confirms that the dissociated $\mathrm{HCO} / \mathrm{HOC}$ molecules have a total NSD of 1, indicating a radical species. NBO provides bond order information throughout the progression of the reaction and confirms bond breakage during the dissociation steps of the reaction mechanism. For example, the $\mathrm{C} 1-\mathrm{C} 2$ bond order drops to zero in the established pathway when $\mathrm{HCO}$ radical dissociates. 


\section{CHAPTER IV}

\section{RESULTS}

\section{Experimental Detection of $\mathrm{HCO}$ in the Reaction of $\mathrm{O}\left({ }^{3} \mathrm{P}\right)+$ Propargyl Alcohol}

The raw data collected via CRDLAS indicates the amount of time it takes for the light of a single laser pulse to decay within the cavity and is known as the ring-down time. The Nd:YAG laser fires at a frequency of $2 \mathrm{~Hz}$ through the dye pump laser, which varies the wavelength by approximately $0.002 \mathrm{~nm}$ every pulse. The lasers must pulse $\sim 1500$ times for a given run to generate a single absorption spectrum for HCO radical. The statistical language program, $\mathrm{R}$, is used to convert ring-down times to absorbance intensities, which is then plotted as a function of wavelength. Figure 8 shows an example of raw, ring-down data collected via CRDLAS for the reaction of $\mathrm{O}\left({ }^{3} \mathrm{P}\right)+$ propargyl alcohol.

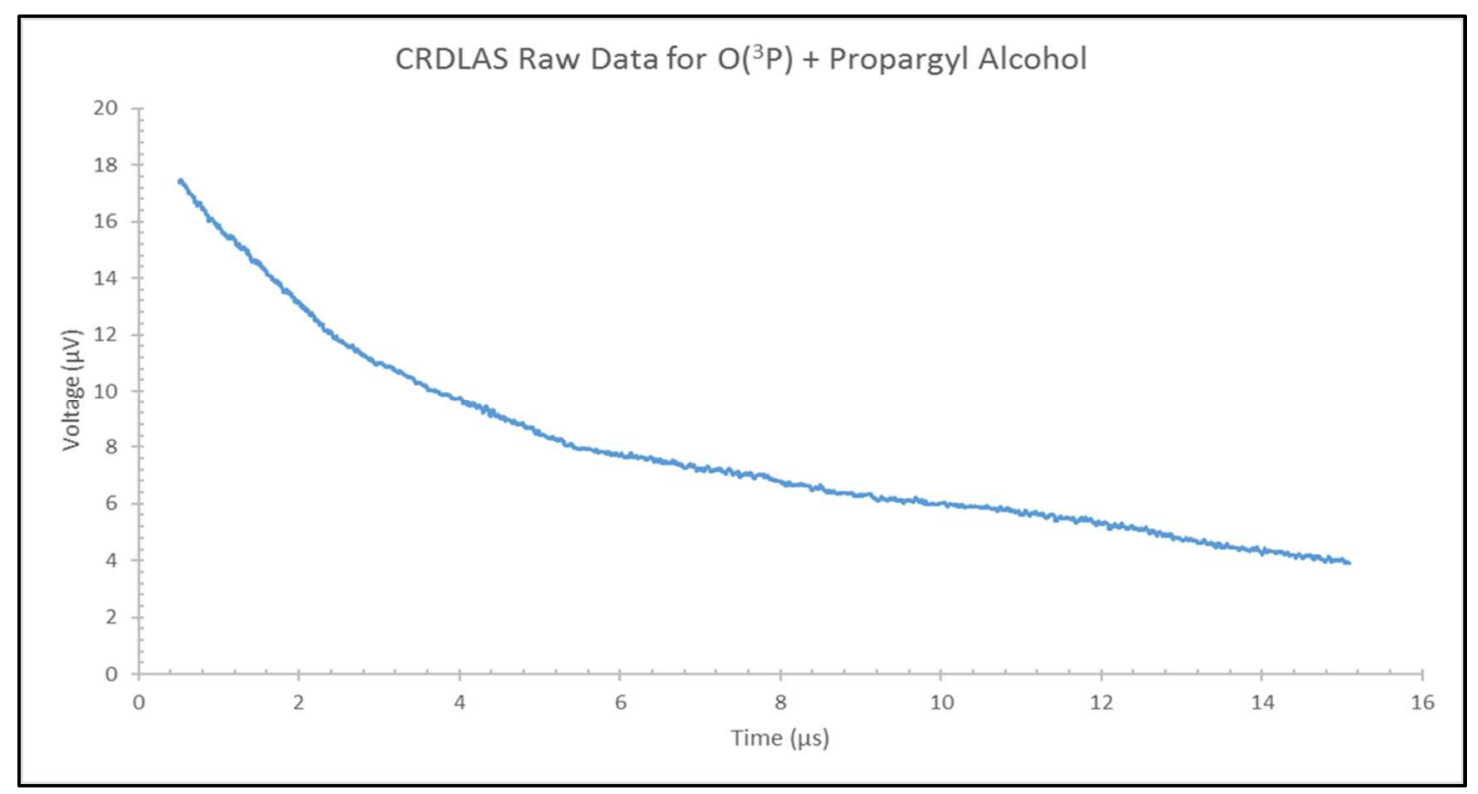

Figure 8. Example for 1 of 1536 data points from a single 613-617 nm scan (ring-down time: $\sim 15 \mu \mathrm{s}$ ). 
HCO radical formation was confirmed for the reaction of $\mathrm{O}\left({ }^{3} \mathrm{P}\right)+$ propargyl alcohol via CRDLAS and its absorption spectrum is shown in Figure 9. The $\tilde{A}^{2} A^{\prime \prime}(0,9,0) \leftarrow \tilde{X}^{2} A^{\prime}(0,0,0)$ transition of HCO radical was observed in the 613-617 nm range with $\mathrm{R}$ and $\mathrm{P}$ branch origins at $614.06 \mathrm{~nm}$ and $614.74 \mathrm{~nm}$, respectively. While ground state HCO radical has a bent geometry, the first excited state of HCO radical takes on a linear conformation, resulting in the observed $\mathrm{P}$ and R branches. The collected spectrum for HCO radical is in good agreement with Buettner's studies and the literature, ${ }^{26}$ confirming the collected spectrum as a signature of $\mathrm{HCO}$ radical.

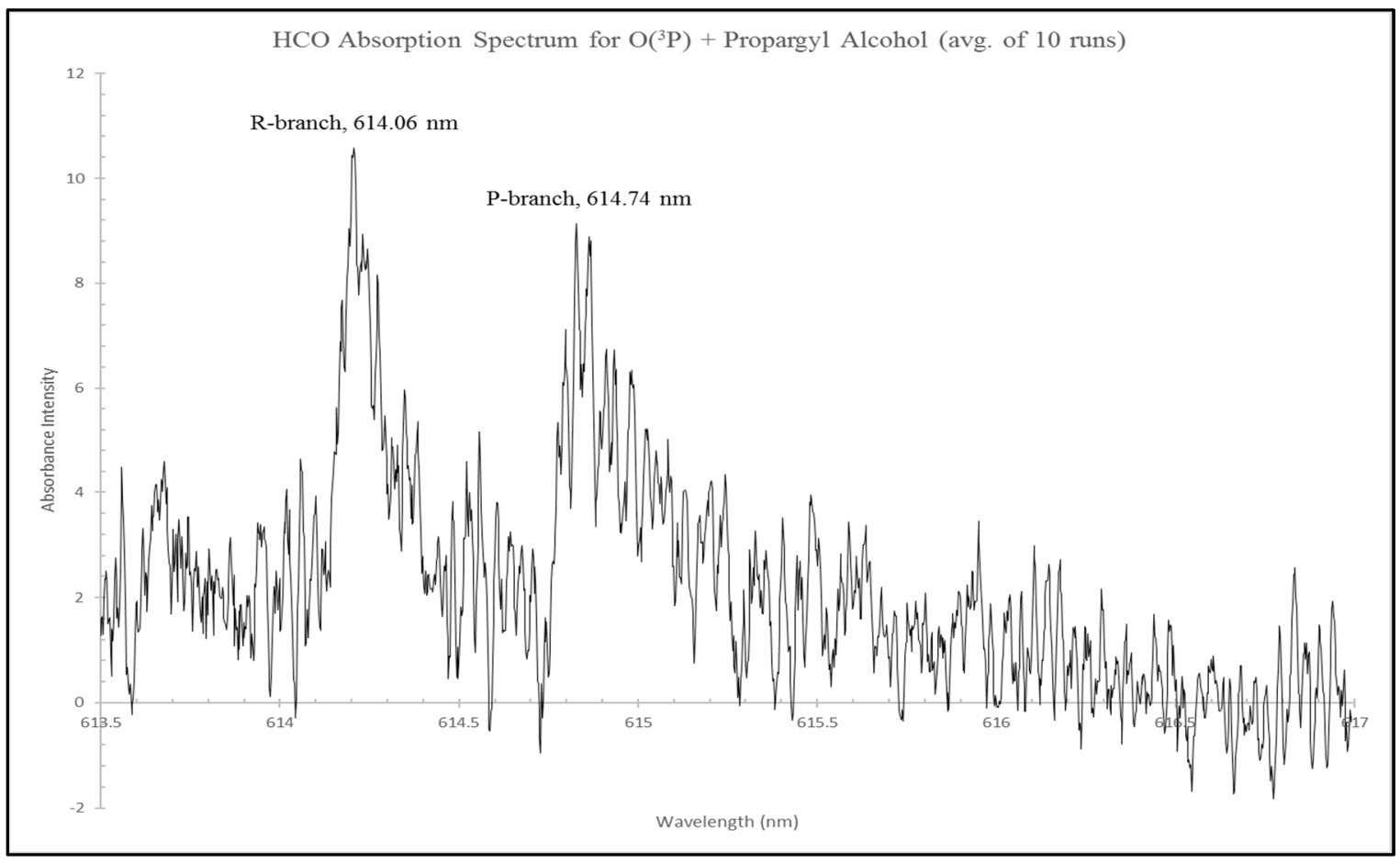

Figure 9. Absorption spectrum for $\mathrm{HCO}$ in the reaction of $\mathrm{O}\left({ }^{3} \mathrm{P}\right)+$ propargyl alcohol. This spectrum is an average of 10 individual runs. 
$\mathrm{HCO}$ radical absorption spectra were collected for other $\mathrm{O}\left({ }^{3} \mathrm{P}\right)+$ alkyne reactions as well to illustrate the varying degrees of intensity observed across different species. During data collection for this project, the $\mathrm{O}\left({ }^{3} \mathrm{P}\right)+$ butyne and $\mathrm{O}\left({ }^{3} \mathrm{P}\right)+$ pentyne reactions were also probed for HCO radical formation and the resultant spectra are shown in Figures 10 and 11. As seen in Figure 11, the $\mathrm{S} / \mathrm{N}$ ratio is too great to confirm the formation of $\mathrm{HCO}$ radical in the reaction of $\mathrm{O}\left({ }^{3} \mathrm{P}\right)+$ pentyne and further experimentation and/or computation is desirable to explain the buried $\mathrm{P}$ and $\mathrm{R}$ branches in the spectrum.

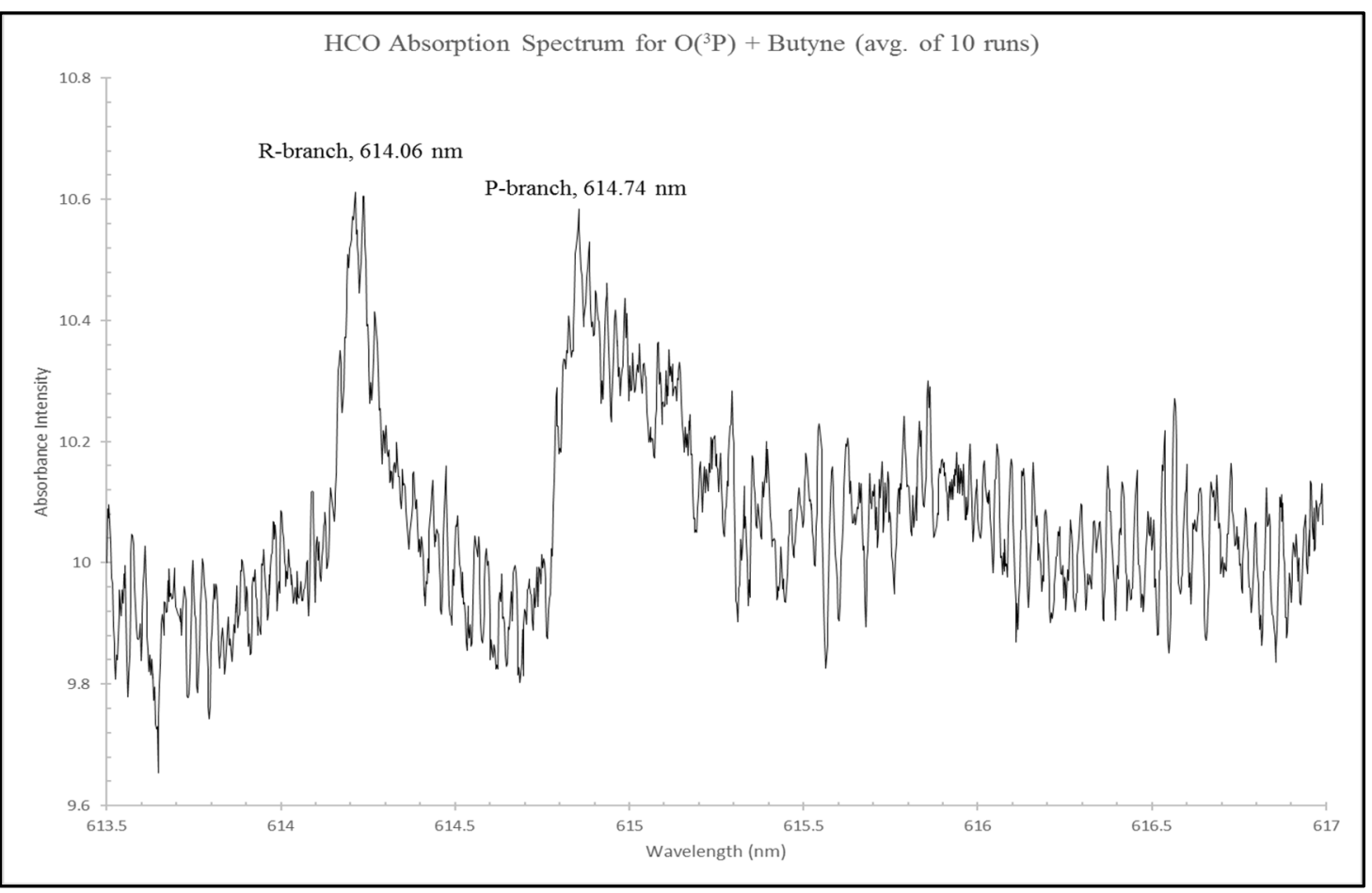

Figure 10. Absorption spectrum for $\mathrm{HCO}$ in the reaction of $\mathrm{O}\left({ }^{3} \mathrm{P}\right)+$ butyne. This spectrum is an average of 10 individual runs. 


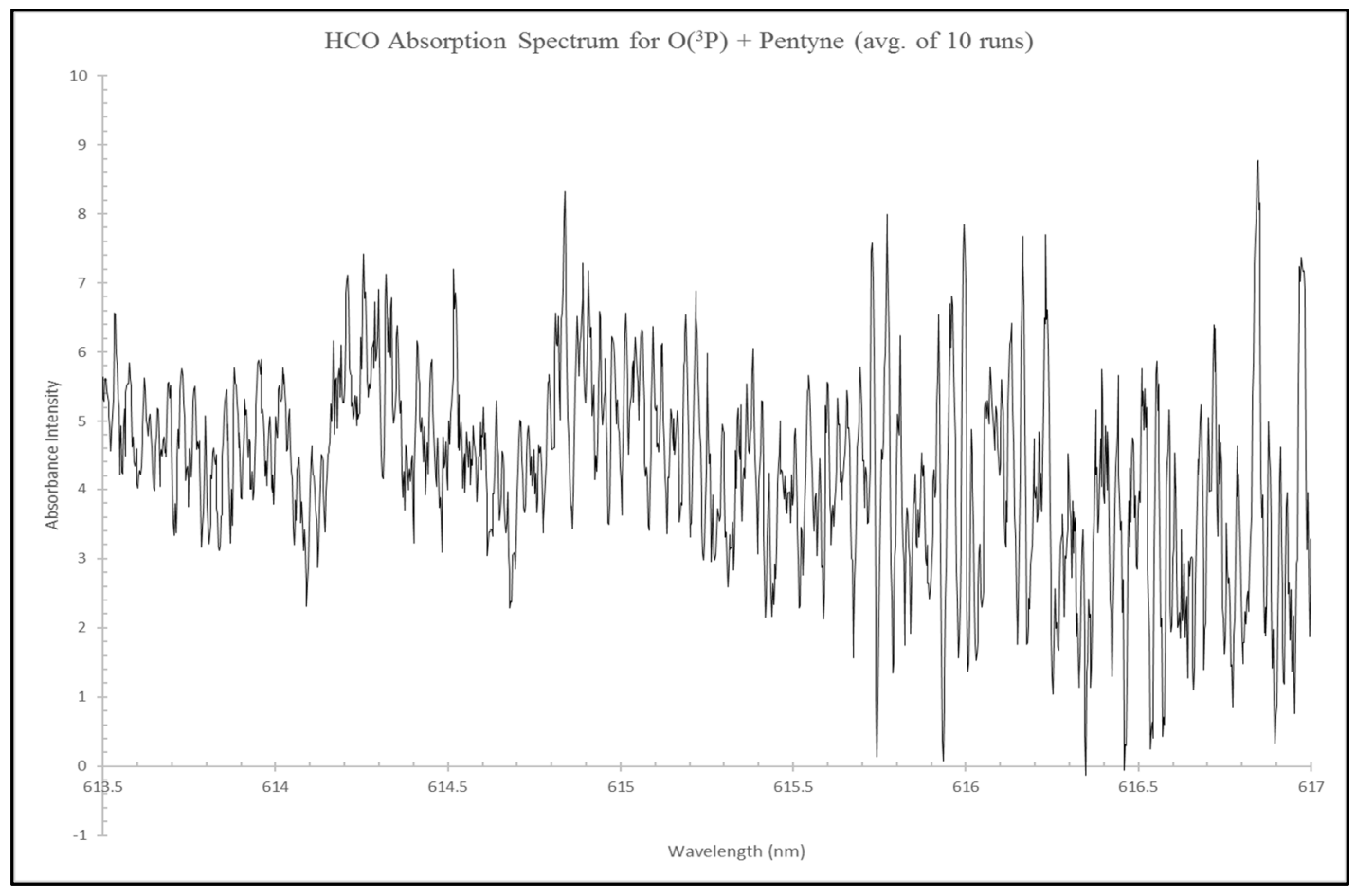

Figure 11. Absorption spectrum for $\mathrm{HCO}$ in the reaction of $\mathrm{O}\left({ }^{3} \mathrm{P}\right)+$ pentyne. This spectrum is an average of 10 individual runs. 
As seen in Figures 9-11, the intensity of HCO radical absorption varies greatly. These variations also were observed in the studies conducted by Buettner and are shown in Figures 12 and 13 .
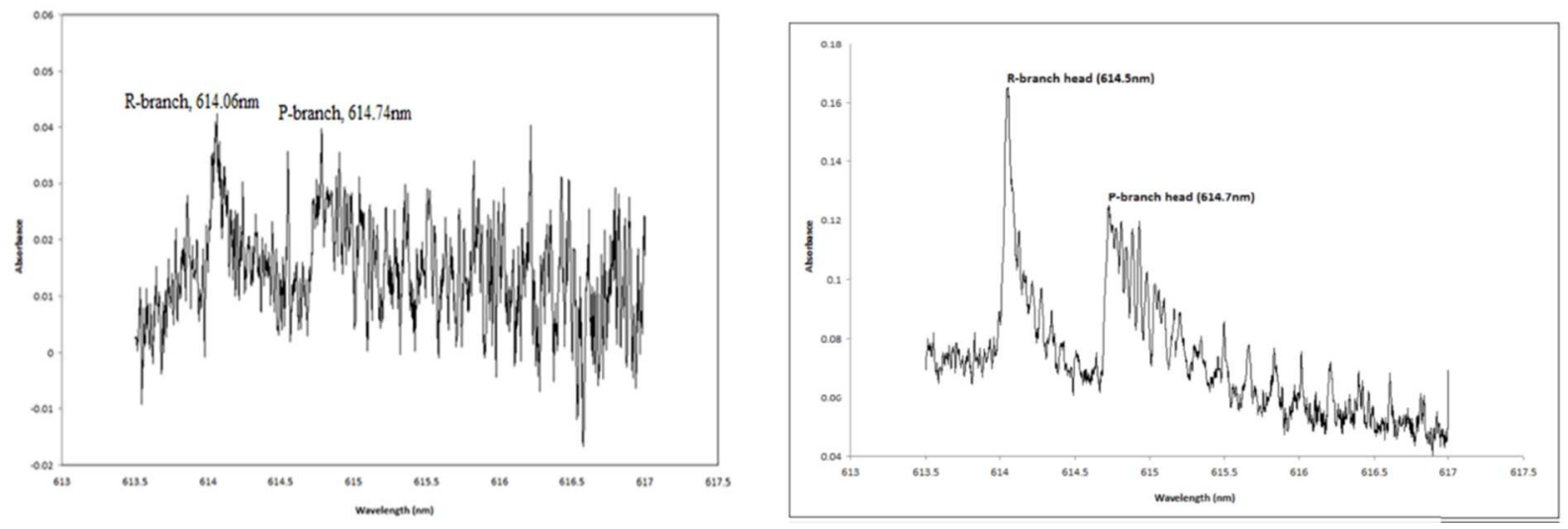

Figure 12. Comparison of absorption spectra for $\mathrm{HCO}$ in the reaction of $\mathrm{O}\left({ }^{3} \mathrm{P}\right)+$ propyne (left) and $\mathrm{O}\left({ }^{3} \mathrm{P}\right)+$ propargyl alcohol (right). Left spectrum is an average of 15 individual runs and the right spectrum is an average of 8 individual runs. ${ }^{28}$
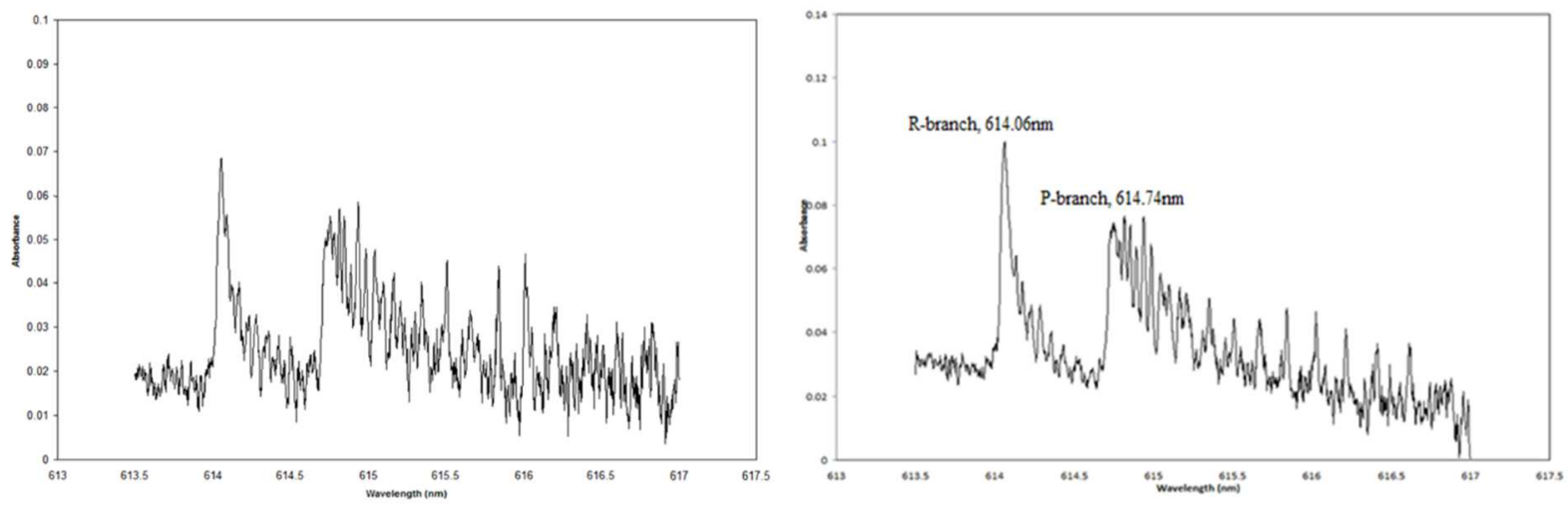

Figure 13. Comparison of absorption spectra for $\mathrm{HCO}$ in the reaction of $\mathrm{O}\left({ }^{3} \mathrm{P}\right)+$ butyne (left) and $\mathrm{O}\left({ }^{3} \mathrm{P}\right)+3$-butyn-1-ol (right). Left spectrum is an average of 10 individual spectra and the right spectrum is an average of 10 individual runs. ${ }^{28}$ 
Hydroxyl-substituted alkynes, when reacted with $\mathrm{O}\left({ }^{3} \mathrm{P}\right)$, produced the strongest absorption signals for HCO radical. The difference in HCO radical absorption intensity observed between $\mathrm{O}\left({ }^{3} \mathrm{P}\right)+$ propyne and $\mathrm{O}\left({ }^{3} \mathrm{P}\right)+$ propargyl alcohol, shown in Figure 12, is worth further discussion. The $\mathrm{HCO}$ radical spectrum for $\mathrm{O}\left({ }^{3} \mathrm{P}\right)+$ propyne was generated by averaging 15 individual runs together, while the $\mathrm{HCO}$ radical spectrum for $\mathrm{O}\left({ }^{3} \mathrm{P}\right)+$ propargyl alcohol is an average of just 8 individual runs. Despite holding the reagent gas ratios constant between each experimental run, the collected spectra for $\mathrm{O}\left({ }^{3} \mathrm{P}\right)+$ propargyl alcohol consistently showed the highest absorption intensity. While it is difficult to quantify the HCO radical product in these reactions, there seems to be a clear, observable difference in the concentration of HCO radical produced between propyne and propargyl alcohol. Up to this point, no plausible explanation for the variations of $\mathrm{HCO}$ radical absorption (shown above) has been proposed.

\section{Computational Results for $\mathbf{O}\left({ }^{3} \mathbf{P}\right)+$ Propargyl Alcohol}

While the results from the computational investigation of the singlet potential energy surface are reported here, only the results from the triplet potential energy surface will be thoroughly discussed as HCO radical is only formed on the triplet surface. The numbering system for $\mathrm{O}\left({ }^{3} \mathrm{P}\right)$ + propargyl alcohol reaction is shown in Figure 14. 


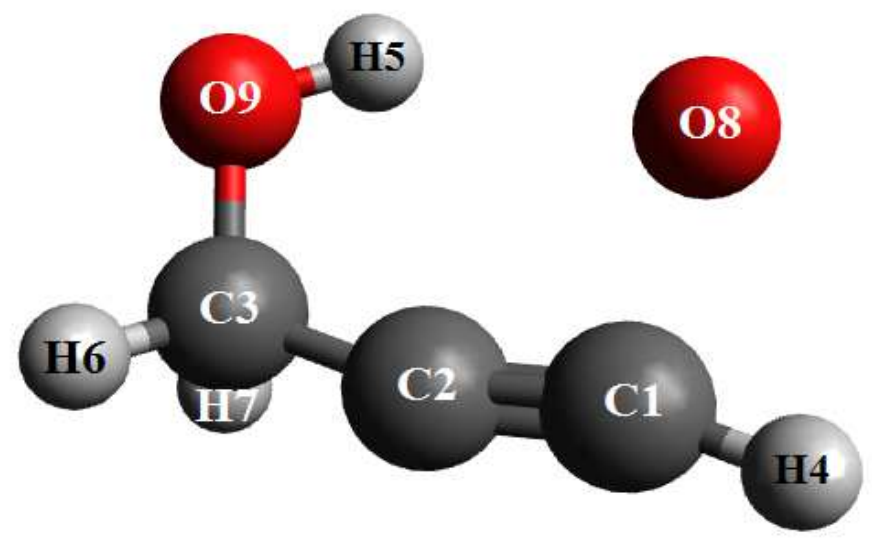

Figure 14. Numbering scheme for the $\mathrm{O}\left({ }^{3} \mathrm{P}\right)+$ propargyl alcohol reaction.

\section{Geometries: $\mathbf{O}\left({ }^{3} \mathbf{P}\right)+$ Propargyl Alcohol}

Relevant bond lengths and angles for each IM and TS on the $\mathrm{O}\left({ }^{3} \mathrm{P}\right)+$ propargyl alcohol triplet and singlet potential energy surfaces have been tabulated and are presented in tables 1-3. All geometries reported here are at the M06-2x/cc-pVTZ and MP2/6-311++G(d,p) levels of theory. Bond lengths agreed well across all optimized M06-2x and MP2 structures on both the triplet and singlet potential energy surfaces, with a maximum difference of just $0.085 \AA$. In general, there is good agreement between the M06-2x and MP2 bond angles with only a few deviations worth noting. First, the optimized structures for IM-5T show a difference of $8.7^{\circ}$ between the M06-2x and MP2 results for the C1-C2-C3 bond angle. Larger geometric deviations are observed on the singlet surface for a few species. TS-2S shows a $16.2^{\circ}$ difference between the M06-2x and MP2 structures for the C1-C2-C3 bond angle and a $15.4^{\circ}$ difference for the $\mathrm{C} 2-$ C1-O8 bond angle. In addition, TS-8S shows a $9^{\circ}$ difference between M06-2x and MP2 for the $\mathrm{C} 1-\mathrm{C} 2-\mathrm{C} 3$ bond angle and an $18.9^{\circ}$ difference for the $\mathrm{C} 2-\mathrm{C} 1-\mathrm{O} 8$ bond angle. 
Table 1. Selected bond lengths and angles for structures on the triplet potential energy surface of $\mathrm{O}\left({ }^{3} \mathrm{P}\right)+$ propargyl alcohol, calculated at the M06-2x/cc-pVTZ and MP2/6-311++G(d,p) levels of theory. The difference between the two levels, $\Delta$, is also reported. The numbering scheme is shown in Figure 14.

\begin{tabular}{|c|c|c|c|c|c|c|c|c|c|c|c|c|}
\hline Species & Level/Basis Set & $\begin{array}{c}\mathrm{C} 1-\mathrm{H} 4 \\
(\AA)\end{array}$ & $\begin{array}{c}\text { C1-C2 } \\
(\AA)\end{array}$ & $\begin{array}{c}\text { C2-C3 } \\
(\AA)\end{array}$ & $\begin{array}{c}\text { C1-O8 } \\
(\AA)\end{array}$ & \begin{tabular}{|c|} 
C3-09 \\
$(\AA)$
\end{tabular} & $\begin{array}{c}\text { O9-H5 } \\
(\AA)\end{array}$ & $\begin{array}{c}<\mathrm{C} 1-\mathrm{C} 2-\mathrm{C} 3 \\
\left({ }^{\circ}\right)\end{array}$ & $\begin{array}{c}<\text { C2-C1-O8 } \\
\left({ }^{\circ}\right)\end{array}$ & $\begin{array}{c}<\mathrm{H} 4-\mathrm{C} 1-\mathrm{O} 8 \\
\left({ }^{\circ}\right)\end{array}$ & $\begin{array}{c}<\mathrm{C} 2-\mathrm{C} 3-\mathrm{O} 9 \\
\left(^{\circ}\right)\end{array}$ & $\begin{array}{c}<\mathrm{C} 3-\mathrm{O} 9-\mathrm{H} 5 \\
\left({ }^{\circ}\right)\end{array}$ \\
\hline \multirow{3}{*}{$\mathrm{CHCCH} 2 \mathrm{OH}$} & \begin{tabular}{|c|} 
M06-2x/cc-pVTZ \\
\end{tabular} & 1.062 & 1.197 & 1.466 & - & 1.412 & \begin{tabular}{|l|}
0.960 \\
\end{tabular} & 178.000 & \begin{tabular}{|l|}
- \\
\end{tabular} & - & 113.000 & 108.300 \\
\hline & MP2/6-311++G(d,p) & 1.065 & 1.218 & 1.466 & - & 1.422 & 0.961 & 179.700 & - & - & 112.800 & 107.000 \\
\hline & \begin{tabular}{|c|}
$\Delta$ \\
\end{tabular} & 0.003 & 0.021 & 0.000 & - & 0.010 & 0.001 & 1.700 & - & - & -0.200 & -1.300 \\
\hline \multirow{3}{*}{ TS-1T } & \begin{tabular}{|l|} 
M06-2x/cc-pVTZ \\
\end{tabular} & 1.064 & 1.218 & 1.464 & 1.972 & 1.408 & 0.965 & 164.100 & 95.600 & 101.200 & 111.300 & 107.600 \\
\hline & MP2/6-311++G(d,p) & 1.066 & 1.211 & 1.463 & 1.847 & 1.416 & 0.965 & 165.500 & 98.400 & 101.300 & 111.600 & 106.500 \\
\hline & $\Delta$ & 0.002 & -0.007 & -0.001 & -0.125 & 0.008 & 0.000 & 1.400 & 2.800 & 0.100 & 0.300 & -1.100 \\
\hline \multirow{3}{*}{ IM-1T } & M06-2x/cc-pVTZ & 1.097 & 1.416 & 1.477 & 1.232 & 1.408 & 0.966 & 129.700 & 120.700 & 120.800 & 112.100 & 107.500 \\
\hline & MP2/6-311++G(d,p) & 1.105 & 1.461 & 1.480 & 1.199 & 1.417 & 0.966 & 125.700 & 120.900 & 122.400 & 111.200 & 105.900 \\
\hline & $\Delta$ & 0.008 & 0.045 & 0.003 & -0.033 & 0.009 & 0.000 & -4.000 & 0.200 & 1.600 & -0.900 & -1.600 \\
\hline \multirow{3}{*}{ TS-2T } & M06-2x/cc-pVTZ & 1.098 & 1.422 & 1.433 & 1.230 & 1.357 & 0.962 & 138.700 & 121.400 & 122.000 & 120.000 & 109.600 \\
\hline & MP2/6-311++G(d,p) & 1.109 & 1.457 & 1.445 & 1.198 & 1.366 & 0.963 & 138.500 & 124.300 & 122.700 & 118.900 & 107.900 \\
\hline & \begin{tabular}{|c|}
$\Delta$ \\
\end{tabular} & 0.011 & 0.035 & 0.012 & -0.032 & 0.009 & 0.001 & -0.200 & 2.900 & 0.700 & -1.100 & -1.700 \\
\hline \multirow{3}{*}{ IM-2T } & M06-2x/cc-pVTZ & 1.103 & 1.442 & 1.461 & 1.219 & 1.364 & 0.961 & 121.300 & 123.000 & 120.600 & 114.400 & 109.600 \\
\hline & MP2/6-311++G(d,p) & 1.112 & 1.481 & 1.465 & 1.190 & 1.370 & 0.962 & 120.800 & 124.100 & 121.800 & 113.500 & 108.400 \\
\hline & \begin{tabular}{|c|}
$\Delta$ \\
\end{tabular} & 0.009 & 0.039 & 0.004 & -0.029 & 0.006 & 0.001 & -0.500 & 1.100 & 1.200 & -0.900 & -1.200 \\
\hline \multirow{3}{*}{ TS-3T } & \begin{tabular}{|l|} 
M06-2x/cc-pVTZ \\
\end{tabular} & - & 1.378 & 1.500 & 1.212 & 1.405 & 0.964 & 132.100 & 135.700 & - & 111.400 & 109.400 \\
\hline & MP2/6-311++G(d,p) & - & 1.393 & 1.508 & 1.201 & 1.413 & 0.964 & 130.600 & 138.500 & - & 110.600 & 108.000 \\
\hline & $\Delta$ & - & 0.015 & 0.008 & -0.011 & 0.008 & 0.000 & -1.500 & 2.800 & - & -0.800 & -1.400 \\
\hline \multirow{3}{*}{ IM-3T } & M06-2x/cc-pVTZ & - & 1.435 & 1.492 & 1.194 & 1.397 & 0.964 & 123.200 & 128.300 & - & 115.800 & 109.300 \\
\hline & MP2/6-311++G(d,p) & - & 1.461 & 1.502 & 1.196 & 1.413 & 0.963 & 120.700 & 127.300 & - & 113.700 & 107.300 \\
\hline & \begin{tabular}{|c|}
$\Delta$ \\
\end{tabular} & - & 0.026 & 0.010 & 0.002 & 0.016 & -0.001 & -2.500 & -1.000 & - & -2.100 & -2.000 \\
\hline \multirow{3}{*}{ TS-4T } & \begin{tabular}{|l|} 
M06-2x/cc-pVTZ \\
\end{tabular} & 1.115 & - & 1.329 & 1.180 & 1.364 & 0.961 & - & - & 125.500 & 122.000 & 109.700 \\
\hline & MP2/6-311++G(d,p) & 1.116 & - & 1.327 & 1.199 & 1.354 & 0.962 & - & - & 125.800 & 121.900 & 108.700 \\
\hline & \begin{tabular}{|c|}
$\Delta$ \\
\end{tabular} & 0.001 & - & -0.002 & 0.019 & -0.010 & 0.001 & - & - & 0.300 & -0.100 & -1.000 \\
\hline \multirow{3}{*}{ TS-5T } & M06-2x/cc-pVTZ & - & - & 1.467 & 1.133 & 1.423 & 0.960 & - & - & - & 109.100 & 108.600 \\
\hline & MP2/6-311++G(d,p) & - & - & 1.477 & 1.148 & 1.431 & 0.961 & - & - & - & 108.700 & 107.000 \\
\hline & \begin{tabular}{|c|}
$\Delta$ \\
\end{tabular} & - & - & 0.010 & 0.015 & 0.008 & 0.001 & - & - & - & -0.400 & -1.600 \\
\hline \multirow{3}{*}{ TS-6T } & M06-2x/cc-pVTZ & - & 1.444 & 1.392 & 1.228 & 1.327 & 0.978 & 121.000 & 127.200 & - & 123.000 & 109.100 \\
\hline & \begin{tabular}{|l|} 
MP2/6-311++G(d,p) \\
\end{tabular} & - & 1.529 & 1.335 & 1.217 & 1.326 & 0.978 & 119.000 & 123.000 & - & 126.900 & 107.300 \\
\hline & $\Delta$ & - & 0.085 & -0.057 & -0.011 & -0.001 & 0.000 & -2.000 & -4.200 & - & 3.900 & -1.800 \\
\hline \multirow{3}{*}{ IM-4T } & M06-2x/cc-pVTZ & - & 1.518 & 1.491 & 1.173 & 1.360 & 0.960 & 114.100 & 128.000 & - & 113.300 & 110.100 \\
\hline & \begin{tabular}{|l|} 
MP2/6-311++G(d,p) \\
\end{tabular} & - & 1.521 & 1.495 & 1.185 & 1.367 & 0.961 & 114.500 & 128.300 & - & 112.800 & 108.700 \\
\hline & $\Delta$ & - & 0.003 & 0.004 & 0.012 & 0.007 & 0.001 & 0.400 & 0.300 & - & -0.500 & -1.400 \\
\hline \multirow{3}{*}{ TS-7T } & M06-2x/cc-pVTZ & - & - & 1.464 & 1.136 & 1.369 & 0.960 & - & - & - & 116.200 & 109.600 \\
\hline & \begin{tabular}{|l|} 
MP2/6-311++G(d,p) \\
\end{tabular} & - & - & 1.468 & 1.150 & 1.376 & 0.962 & - & - & - & 114.200 & 108.100 \\
\hline & \begin{tabular}{|c|}
$\Delta$ \\
\end{tabular} & - & - & 0.004 & 0.014 & 0.007 & 0.002 & - & - & - & -2.000 & -1.500 \\
\hline \multirow{3}{*}{ TS-8T } & M06-2x/cc-pVTZ & 1.085 & 1.373 & 1.500 & 1.307 & 1.362 & - & 123.400 & 122.600 & 115.100 & 99.000 & - \\
\hline & \begin{tabular}{|l|} 
MP2/6-311++G(d,p) \\
\end{tabular} & \multicolumn{11}{|c|}{ Did not converge } \\
\hline & $\Delta$ & \multicolumn{11}{|c|}{ 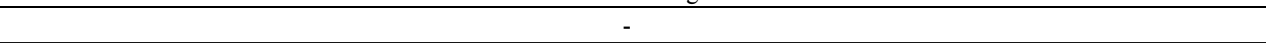 } \\
\hline \multirow{3}{*}{ IM-5T } & M06-2x/cc-pVTZ & 1.083 & 1.322 & 1.483 & 1.355 & 1.386 & - & 130.000 & 124.800 & 112.300 & 103.400 & - \\
\hline & \begin{tabular}{|l|} 
MP2/6-311++G(d,p) \\
\end{tabular} & 1.085 & 1.296 & 1.479 & 1.364 & 1.389 & - & 138.700 & 128.500 & 110.300 & 106.200 & - \\
\hline & $\Delta$ & 0.002 & -0.026 & -0.004 & 0.009 & 0.003 & - & 8.700 & 3.700 & -2.000 & 2.800 & - \\
\hline \multirow{3}{*}{ TS-9T } & M06-2x/cc-pVTZ & 1.081 & 1.375 & 1.450 & 1.320 & 1.275 & - & 124.300 & 117.400 & 116.400 & 117.400 & - \\
\hline & \begin{tabular}{|l|} 
MP2/6-311++G(d,p) \\
\end{tabular} & 1.083 & 1.381 & 1.460 & 1.325 & 1.280 & - & 124.200 & 120.000 & 116.100 & 117.400 & - \\
\hline & $\Delta$ & 0.002 & 0.006 & 0.010 & 0.005 & 0.005 & - & -0.100 & 2.600 & -0.300 & 0.000 & - \\
\hline \multirow{3}{*}{ IM-6T } & M06-2x/cc-pVTZ & 1.076 & 1.462 & 1.404 & 1.329 & 1.267 & - & 123.500 & 119.700 & 117.000 & 121.000 & - \\
\hline & \begin{tabular}{|l|}
$\mathrm{MP} 2 / 6-311++\mathrm{G}(\mathrm{d}, \mathrm{p})$ \\
\end{tabular} & 1.082 & 1.484 & 1.464 & 1.363 & 1.201 & - & 118.600 & 114.500 & 113.500 & 122.200 & - \\
\hline & $\Delta$ & 0.006 & 0.022 & 0.060 & 0.034 & -0.066 & - & -4.900 & -5.200 & -3.500 & 1.200 & - \\
\hline
\end{tabular}


Table 2. Continued bond lengths and angles for structures on the triplet potential energy surface of $\mathrm{O}\left({ }^{3} \mathrm{P}\right)+$ propargyl alcohol, calculated at the M06-2x/cc-pVTZ and MP2/6-311++G(d,p) levels of theory. The difference between the two levels, $\Delta$, is also reported. The numbering scheme is shown in Figure 14.

\begin{tabular}{|c|c|c|c|c|c|c|c|c|c|c|c|c|}
\hline Species & Leve/Basis Set & $\begin{array}{c}\text { C1-H4 } \\
(\AA)\end{array}$ & $\begin{array}{c}\mathrm{C} 1-\mathrm{C} 2 \\
(\AA)\end{array}$ & $\begin{array}{c}\mathrm{C} 2-\mathrm{C} 3 \\
(\AA)\end{array}$ & $\begin{array}{c}\mathrm{C} 1-08 \\
(\AA)\end{array}$ & \begin{tabular}{|c|}
$\begin{array}{c}\text { C3-O9 } \\
(\AA)\end{array}$ \\
\end{tabular} & \begin{tabular}{|c|} 
O9-H5 \\
$(\AA)$
\end{tabular} & $\begin{array}{c}<\mathrm{C} 1-\mathrm{C} 2-\mathrm{C} 3 \\
\left({ }^{\circ}\right)\end{array}$ & $\begin{array}{c}<\text { C2-C1-O8 } \\
\left({ }^{\circ}\right)\end{array}$ & \begin{tabular}{|c}
$<\mathrm{H} 4-\mathrm{Cl}-\mathrm{O} 8$ \\
$\left({ }^{\circ}\right)$
\end{tabular} & $\begin{array}{c}<\mathrm{C} 2-\mathrm{C} 3-\mathrm{O} 9 \\
\left({ }^{\circ}\right)\end{array}$ & $\begin{array}{c}<\mathrm{C} 3-\mathrm{O} 9-\mathrm{H} 5 \\
\left({ }^{\circ}\right)\end{array}$ \\
\hline \multirow{3}{*}{ TS-10T } & M06-2x/cc-pVTZ & 1.089 & 1.315 & - & 1.342 & 1.190 & \begin{tabular}{|l|}
- \\
\end{tabular} & - & 128.500 & \begin{tabular}{|l|}
111.900 \\
\end{tabular} & - & - \\
\hline & MP2/6-311++G(d,p) & 1.092 & 1.307 & - & 1.336 & 1.202 & - & - & 129.600 & 111.800 & - & - \\
\hline & $\Delta$ & 0.003 & -0.008 & - & -0.006 & 0.012 & - & - & 1.100 & -0.100 & - & - \\
\hline \multirow{3}{*}{ TS-11T } & M06-2x/cc-pVTZ & 1.102 & 1.444 & 1.458 & 1.219 & 1.335 & 0.964 & 120.700 & 123.500 & 121.600 & 125.500 & 111.200 \\
\hline & MP2/6-311++G(d,p) & 1.111 & 1.462 & 1.459 & 1.206 & 1.342 & 0.964 & 119.900 & 124.800 & 122.200 & 125.400 & 109.200 \\
\hline & $\Delta$ & 0.009 & 0.018 & 0.001 & -0.013 & 0.007 & 0.000 & -0.800 & 1.300 & 0.600 & -0.100 & -2.000 \\
\hline \multirow{3}{*}{ IM-7T } & M06-2x/cc-pVTZ & 1.106 & 1.506 & 1.478 & 1.197 & 1.338 & 0.965 & 112.900 & 124.500 & 120.900 & 124.200 & 111.300 \\
\hline & MP2/6-311++G(d,p) & 1.109 & 1.510 & 1.488 & 1.211 & 1.349 & 0.964 & 113.600 & 125.100 & 120.900 & 122.800 & 109.300 \\
\hline & $\Delta$ & 0.003 & 0.004 & 0.010 & 0.014 & 0.011 & -0.001 & 0.700 & 0.600 & 0.000 & -1.400 & -2.000 \\
\hline \multirow{3}{*}{ TS-12T } & M06-2x/cc-pVTZ & 1.100 & 1.441 & - & 1.223 & 1.270 & 0.985 & - & 123.300 & 120.300 & - & 113.000 \\
\hline & MP2/6-311++G(d,p) & 1.108 & 1.469 & - & 1.205 & 1.273 & 0.986 & - & 124.400 & 121.200 & - & 110.100 \\
\hline & $\Delta$ & 0.008 & 0.028 & - & -0.018 & 0.003 & 0.001 & - & 1.100 & 0.900 & - & -2.900 \\
\hline \multirow{3}{*}{ TS-13T } & M06-2x/cc-pVTZ & - & 1.471 & 1.495 & 1.265 & 1.260 & - & 110.500 & 121.300 & - & 120.000 & - \\
\hline & MP2/6-311++G(d,p) & \multicolumn{11}{|c|}{ Did not converge. } \\
\hline & $\Delta$ & \multicolumn{11}{|c|}{-} \\
\hline \multirow{3}{*}{$\mathrm{IM}-8 \mathrm{~T}$} & M06-2x/cc-pVTZ & - & 1.478 & 1.514 & 1.331 & 1.201 & - & 111.800 & 124.000 & - & 123.900 & - \\
\hline & MP2/6-311++G(d,p) & - & 1.489 & 1.517 & 1.340 & 1.214 & - & 112.200 & 123.600 & - & 124.100 & - \\
\hline & $\Delta$ & - & 0.011 & 0.003 & 0.009 & 0.013 & - & 0.400 & -0.400 & - & 0.200 & - \\
\hline \multirow{3}{*}{ TS-14T } & M06-2x/cc-pVTZ & - & - & 1.441 & 1.270 & 1.223 & - & - & - & - & 123.300 & - \\
\hline & MP2/6-311++G(d,p) & - & - & 1.469 & 1.273 & 1.205 & - & - & - & - & 124.400 & - \\
\hline & $\Delta$ & - & - & 0.028 & 0.003 & -0.018 & - & - & - & - & 1.100 & - \\
\hline \multirow{3}{*}{ TS-15T } & M06-2x/cc-pVTZ & - & 1.341 & - & 1.305 & 1.194 & - & - & 130.100 & - & - & - \\
\hline & MP2/6-311++G(d,p) & - & 1.339 & - & 1.285 & 1.222 & - & - & 129.900 & - & - & - \\
\hline & $\Delta$ & - & -0.002 & - & -0.020 & 0.028 & - & - & -0.200 & - & - & - \\
\hline \multirow{3}{*}{ TS-16T } & M06-2x/cc-pVTZ & - & 1.497 & 1.533 & 1.261 & 1.335 & 0.965 & 109.200 & 110.900 & - & 121.500 & 111.400 \\
\hline & MP2/6-311++G(d,p) & \multicolumn{11}{|c|}{ Did not converge. } \\
\hline & $\Delta$ & \\
\hline \multirow{3}{*}{ IM-9T } & M06-2x/cc-pVTZ & - & 1.528 & 1.489 & 1.289 & 1.339 & 0.965 & 117.700 & 111.900 & - & 123.700 & 111.300 \\
\hline & MP2/6-311++G(d,p) & - & 1.535 & 1.497 & 1.298 & 1.348 & 0.964 & 116.700 & 111.600 & - & 122.500 & 109.500 \\
\hline & $\Delta$ & - & 0.007 & 0.008 & 0.009 & 0.009 & -0.001 & -1.000 & -0.300 & - & -1.200 & -1.800 \\
\hline \multirow{3}{*}{ TS-17T-a } & M06-2x/cc-pVTZ & - & - & 1.357 & 1.250 & 1.345 & 0.964 & - & - & - & 127.700 & 110.100 \\
\hline & MP2/6-311++G(d,p) & - & - & 1.347 & 1.258 & 1.351 & 0.964 & - & - & - & 127.700 & 108.400 \\
\hline & $\Delta$ & - & - & -0.010 & 0.008 & 0.006 & 0.000 & - & - & - & 0.000 & -1.700 \\
\hline \multirow{3}{*}{ TS-17T-b } & M06-2x/cc-pVTZ & - & 1.361 & - & 1.269 & 1.285 & 0.972 & - & 126.800 & - & - & 113.300 \\
\hline & MP2/6-311++G(d,p) & - & 1.348 & - & 1.273 & 1.281 & 0.974 & - & 128.900 & - & - & 111.400 \\
\hline & $\Delta$ & - & -0.013 & - & 0.004 & -0.004 & 0.002 & - & 2.100 & - & - & -1.900 \\
\hline \multirow{3}{*}{ TS-18T } & M06-2x/cc-pVTZ & 1.095 & 1.480 & 1.350 & 1.253 & 1.347 & 0.960 & 130.200 & 118.900 & 123.200 & 121.400 & 109.900 \\
\hline & MP2/6-311++G(d,p) & & & & & & & id not converge & & & & \\
\hline & $\Delta$ & & & & & & & - & & & & \\
\hline & M06-2x/cc-pVTZ & 1.102 & 1.477 & 1.308 & 1.374 & 1.364 & 0.959 & 134.900 & 114.300 & 107.100 & 123.600 & 109.200 \\
\hline IM-10T & MP2/6-311++G(d,p) & 1.100 & 1.480 & 1.291 & 1.388 & 1.368 & 0.959 & 137.000 & 113.800 & 106.800 & 124.500 & 108.000 \\
\hline & $\Delta$ & -0.002 & 0.003 & -0.017 & 0.014 & 0.004 & 0.000 & 2.100 & -0.500 & -0.300 & 0.900 & -1.200 \\
\hline & M06-2x/cc-pVTZ & 1.103 & 1.497 & 1.331 & 1.373 & 1.344 & 0.961 & 126.600 & 117.500 & 111.700 & 125.900 & 108.200 \\
\hline TS-19T & MP2/6-311++G(d,p) & 1.103 & 1.496 & 1.331 & 1.383 & 1.358 & 0.961 & 131.600 & 116.200 & 111.700 & 123.700 & 106.100 \\
\hline & $\Delta$ & 0.000 & -0.001 & 0.000 & 0.010 & 0.014 & 0.000 & 5.000 & -1.300 & 0.000 & -2.200 & -2.100 \\
\hline & M06-2x/cc-pVTZ & 1.104 & 1.502 & 1.313 & 1.361 & 1.329 & 0.963 & 122.700 & 116.400 & 107.200 & 130.600 & 110.100 \\
\hline IM-11T & MP2/6-311++G(d,p) & 1.103 & 1.504 & 1.298 & 1.373 & 1.328 & 0.963 & 123.800 & 116.000 & 108.000 & 131.700 & 108.500 \\
\hline & $\Delta$ & -0.001 & 0.002 & -0.015 & 0.012 & -0.001 & 0.000 & 1.100 & -0.400 & 0.800 & 1.100 & -1.600 \\
\hline & M06-2x/cc-pVTZ & 1.101 & - & 1.311 & 1.215 & 1.327 & 0.965 & - & - & 121.400 & 128.600 & 109.900 \\
\hline TS-20T & MP2/6-311++G(d,p) & 1.109 & - & 1.327 & 1.205 & 1.329 & 0.965 & - & - & 121.900 & 128.100 & 108.300 \\
\hline & $\Delta$ & 0.008 & - & 0.016 & -0.010 & 0.002 & 0.000 & - & - & 0.500 & -0.500 & -1.600 \\
\hline
\end{tabular}


Table 3. Selected bond lengths and angles for structures on the singlet potential energy surface of $\mathrm{O}\left({ }^{3} \mathrm{P}\right)+$ propargyl alcohol, calculated at the M06-2x/cc-pVTZ and MP2/6-311++G(d,p) levels of theory. The difference between the two levels, $\Delta$, is also reported. The numbering scheme is shown in Figure 14.

\begin{tabular}{|c|c|c|c|c|c|c|c|c|c|c|c|c|}
\hline Species & Level/Basis Set & $\begin{array}{c}\mathrm{C} 1-\mathrm{H} 4 \\
(\AA) \\
\end{array}$ & $\begin{array}{c}\mathrm{C} 1-\mathrm{C} 2 \\
(\AA) \\
\end{array}$ & $\begin{array}{c}\mathrm{C} 2-\mathrm{C} 3 \\
(\AA)\end{array}$ & $\begin{array}{c}\text { C1-08 } \\
(\AA) \\
\end{array}$ & $\begin{array}{c}\text { C3-09 } \\
(\AA)\end{array}$ & $\begin{array}{c}\text { O9-H5 } \\
(\AA) \\
\end{array}$ & $\begin{array}{c}<\mathrm{C} 1-\mathrm{C} 2-\mathrm{C} 3 \\
\left({ }^{\circ}\right)\end{array}$ & $\begin{array}{c}<\mathrm{C} 2-\mathrm{C} 1-08 \\
\left({ }^{\circ}\right) \\
\end{array}$ & $\begin{array}{c}<\mathrm{H} 4-\mathrm{Cl}-\mathrm{O} 8 \\
\left({ }^{\circ}\right)\end{array}$ & $\begin{array}{c}<\mathrm{C} 2-\mathrm{C} 3-09 \\
\left({ }^{\circ}\right)\end{array}$ & $\begin{array}{c}<\mathrm{C} 3-09-\mathrm{H} 5 \\
\left({ }^{\circ}\right)\end{array}$ \\
\hline \multirow{3}{*}{$\mathrm{CHCCH} 2 \mathrm{OH}$} & M06-2x/cc-pVTZ & 1.062 & 1.197 & 1.466 & - & 1.412 & 0.960 & 178.000 & - & - & 113.000 & 108.300 \\
\hline & MP2/6-311++G(d,p) & 1.065 & 1.218 & 1.466 & - & 1.422 & 0.961 & 179.700 & - & - & 112.800 & 107.000 \\
\hline & $\Delta$ & 0.003 & 0.021 & 0.000 & - & 0.010 & 0.001 & 1.700 & - & - & -0.200 & -1.300 \\
\hline \multirow{3}{*}{ IM-1S } & M06-2x/cc-pVTZ & 1.101 & 1.423 & 1.448 & 1.223 & 1.404 & 0.964 & 117.700 & 115.000 & 123.300 & 120.100 & 108.100 \\
\hline & MP2/6-311++G(d,p) & 1.103 & 1.433 & 1.451 & 1.237 & 1.414 & 0.963 & 117.200 & 113.800 & 123.600 & 120.800 & 107.000 \\
\hline & \begin{tabular}{|l|}
$\Delta$ \\
\end{tabular} & 0.002 & 0.010 & 0.003 & 0.014 & 0.010 & -0.001 & -0.500 & -1.200 & 0.300 & 0.700 & -1.100 \\
\hline \multirow{3}{*}{ TS- $2 \mathrm{~S}$} & M06-2x/cc-pVTZ & 1.098 & 1.422 & 1.433 & 1.230 & 1.357 & 0.962 & 138.700 & 121.400 & 122.000 & 120.000 & 109.600 \\
\hline & MP2/6-311++G(d,p) & 1.139 & 1.411 & 1.447 & 1.220 & 1.411 & 0.962 & 122.500 & 136.800 & 122.500 & 122.400 & 108.800 \\
\hline & \begin{tabular}{|c|}
$\Delta$ \\
\end{tabular} & 0.041 & -0.011 & 0.014 & -0.010 & 0.054 & 0.000 & -16.200 & 15.400 & 0.500 & 2.400 & -0.800 \\
\hline \multirow{3}{*}{ IM-2S } & M06-2x/cc-pVTZ & 1.100 & 1.439 & 1.355 & 1.228 & 1.314 & 0.993 & 119.100 & 123.600 & 118.900 & 124.400 & 106.500 \\
\hline & MP2/6-311++G(d,p) & 1.102 & 1.443 & 1.366 & 1.241 & 1.323 & 0.992 & 119.400 & 123.300 & 119.100 & 124.200 & 105.200 \\
\hline & \begin{tabular}{|l|}
$\Delta$ \\
\end{tabular} & 0.002 & 0.004 & 0.011 & 0.013 & 0.009 & -0.001 & 0.300 & -0.300 & 0.200 & -0.200 & -1.300 \\
\hline \multirow{3}{*}{ TS-3S } & M06-2x/cc-pVTZ & 1.141 & 1.398 & 1.428 & 1.205 & 1.394 & 0.963 & 120.800 & 140.000 & 122.500 & 123.700 & 109.700 \\
\hline & \begin{tabular}{|l|} 
MP2/6-311++G(d,p) \\
\end{tabular} & 1.152 & 1.401 & 1.434 & 1.216 & 1.401 & 0.963 & 120.400 & 142.400 & 123.400 & 124.200 & 108.500 \\
\hline & $\Delta$ & 0.011 & 0.003 & 0.006 & 0.011 & 0.007 & 0.000 & -0.400 & 2.400 & 0.900 & 0.500 & -1.200 \\
\hline \multirow{3}{*}{ IM-3S } & M06-2x/cc-pVTZ & - & 1.311 & 1.508 & 1.154 & 1.414 & 0.960 & 121.400 & 178.800 & - & 114.200 & 108.700 \\
\hline & MP2/6-311++G(d,p) & - & 1.326 & 1.506 & 1.166 & 1.426 & 0.961 & 121.000 & 178.600 & - & 114.200 & 106.800 \\
\hline & $\Delta$ & - & 0.015 & -0.002 & 0.012 & 0.012 & 0.001 & -0.400 & -0.200 & - & 0.000 & -1.900 \\
\hline \multirow{3}{*}{ TS-6S } & M06-2x/cc-pVTZ & - & - & 1.405 & 1.126 & 1.384 & 0.961 & - & - & - & 123.000 & 109.900 \\
\hline & MP2/6-311++G(d,p) & - & - & 1.424 & 1.140 & 1.388 & 0.966 & - & - & - & 119.600 & 105.400 \\
\hline & \begin{tabular}{|c|}
$\Delta$ \\
\end{tabular} & - & - & 0.019 & 0.014 & 0.004 & 0.005 & - & - & - & -3.400 & -4.500 \\
\hline \multirow{3}{*}{ TS-8S } & M06-2x/cc-pVTZ & - & 1.400 & 1.428 & 1.163 & 1.377 & 0.961 & 107.400 & 160.900 & - & 117.700 & 110.500 \\
\hline & MP2/6-311++G(d,p) & - & 1.448 & 1.424 & 1.194 & 1.365 & 0.962 & 116.400 & 142.000 & - & 117.900 & 109.100 \\
\hline & \begin{tabular}{|l|}
$\Delta$ \\
\end{tabular} & - & 0.048 & -0.004 & 0.031 & -0.012 & 0.001 & 9.000 & -18.900 & - & 0.200 & -1.400 \\
\hline \multirow{3}{*}{ TS-19S } & M06-2x/cc-pVTZ & 1.147 & 1.389 & 1.433 & 1.202 & 1.392 & 0.963 & 122.600 & 141.500 & 122.600 & 118.800 & 107.900 \\
\hline & MP2/6-311++G(d,p) & \multicolumn{11}{|c|}{ Did not converge. } \\
\hline & \begin{tabular}{|l|}
$\Delta$ \\
\end{tabular} & \multicolumn{11}{|c|}{-} \\
\hline \multirow{3}{*}{ TS-10S } & M06-2x/cc-pVTZ & - & - & 1.235 & 1.137 & 1.319 & 0.962 & - & - & - & 153.500 & 110.900 \\
\hline & MP2/6-311++G(d,p) & - & - & 1.257 & 1.155 & 1.324 & 0.963 & - & - & - & 154.700 & 109.500 \\
\hline & \begin{tabular}{|l|}
$\Delta$ \\
\end{tabular} & - & - & 0.022 & 0.018 & 0.005 & 0.001 & - & - & - & 1.200 & -1.400 \\
\hline \multirow{3}{*}{ TS-11S } & M06-2x/cc-pVTZ & - & 1.436 & 1.480 & 1.159 & 1.397 & 0.960 & 87.500 & 167.600 & - & 115.500 & 108.400 \\
\hline & MP2/6-311++G(d,p) & - & 1.426 & 1.498 & 1.177 & 1.407 & 0.960 & 85.800 & 174.100 & - & 115.000 & 106.700 \\
\hline & $\Delta$ & - & -0.010 & 0.018 & 0.018 & 0.010 & 0.000 & -1.700 & 6.500 & - & -0.500 & -1.700 \\
\hline
\end{tabular}




\section{Potential Energy Profiles: $\mathbf{O}\left({ }^{3} \mathbf{P}\right)+$ Propargyl Alcohol}

All potential energies presented in this section are referenced relative to the reactants of the reaction, propargyl alcohol and $\mathrm{O}\left({ }^{3} \mathrm{P}\right)$. Computational work by Buettner has explored the potential energy profiles (PEPs) for the reactions of various alkynes and substituted alkynes with $\mathrm{O}\left({ }^{3} \mathrm{P}\right)$ at the MP2/6-311++G(d,p) level of theory. ${ }^{28}$ Dilday's investigation of $\mathrm{O}\left({ }^{3} \mathrm{P}\right)+$ propyne demonstrated that the M06-2x functional provides relative energies closer to the $\operatorname{CCSD}(\mathrm{T})$ single point results than does MP2. ${ }^{29}$ Triplet surface relative energies at the MP2(Full), M06-2x, and $\operatorname{CCSD}(\mathrm{T})$ single point levels of theory are reported in tables $1-5$, which confirms the previous observations that M06-2x energies agree better with $\operatorname{CCSD}(\mathrm{T})$ single-point energies than does MP2. For this reason, all single-point energy calculations at the $\operatorname{CCSD}(\mathrm{T}) / \mathrm{cc}-\mathrm{pVTZ}$ level of theory were carried out on the M06-2x/cc-pVTZ optimized geometries. All energies reported in this thesis are corrected for vibrational zero-point energies unless stated otherwise. 
Table 4. Relative energies for structures on the triplet potential energy surface, computed using the M06-2x, MP2, and CCSD(T)//M06-2x single point methods. Relative energy differences, $\Delta$, for M06-2x and MP2 results compared to $\operatorname{CCSD}(\mathrm{T})$ single-point results are also shown.

\begin{tabular}{|c|c|c|}
\hline Species & Level of Theory & Rel. E (kJ/mol) \\
\hline \multirow{5}{*}{ TS-1T } & M06-2x & -7.25 \\
\hline & MP2(Full) & 77.41 \\
\hline & CCSD(T) Single Point & 10.40 \\
\hline & $\Delta: \mathrm{M} 06-2 \mathrm{x}-\mathrm{CCSD}(\mathrm{T})$ & -17.65 \\
\hline & $\Delta: \mathrm{MP} 2(\mathrm{Full})-\mathrm{CCSD}(\mathrm{T})$ & 67.02 \\
\hline \multirow{5}{*}{ IM-1T } & M06-2x & -248.96 \\
\hline & MP2(Full) & -187.50 \\
\hline & CCSD(T) Single Point & -220.27 \\
\hline & $\Delta: \mathrm{M} 06-2 \mathrm{x}-\mathrm{CCSD}(\mathrm{T})$ & -28.69 \\
\hline & $\Delta:$ MP2(Full) - CCSD(T) & 32.77 \\
\hline \multirow{5}{*}{ TS-2T } & M06-2x & -63.33 \\
\hline & MP2(Full) & 9.66 \\
\hline & CCSD(T) Single Point & -30.60 \\
\hline & $\Delta: \mathrm{M} 06-2 \mathrm{x}-\mathrm{CCSD}(\mathrm{T})$ & -32.73 \\
\hline & $\Delta: \mathrm{MP} 2(\mathrm{Full})-\mathrm{CCSD}(\mathrm{T})$ & 40.25 \\
\hline \multirow{5}{*}{ IM-2T } & M06-2x & -278.32 \\
\hline & MP2(Full) & -222.21 \\
\hline & CCSD(T) Single Point & -255.02 \\
\hline & $\Delta: \mathrm{M} 06-2 \mathrm{x}-\mathrm{CCSD}(\mathrm{T})$ & -23.29 \\
\hline & $\Delta:$ MP2(Full) - CCSD(T) & 32.81 \\
\hline \multirow{5}{*}{ TS-3T } & M06-2x & -60.58 \\
\hline & MP2(Full) & -154.84 \\
\hline & CCSD(T) Single Point & -35.52 \\
\hline & $\Delta: \mathrm{M} 06-2 \mathrm{x}-\mathrm{CCSD}(\mathrm{T})$ & -25.06 \\
\hline & $\Delta:$ MP2(Full) - CCSD(T) & -119.32 \\
\hline \multirow{5}{*}{ IM-3T } & M06-2x & -309.97 \\
\hline & MP2(Full) & -280.51 \\
\hline & CCSD(T) Single Point & -288.26 \\
\hline & $\Delta: \mathrm{M} 06-2 \mathrm{x}-\mathrm{CCSD}(\mathrm{T})$ & -21.71 \\
\hline & $\Delta: \mathrm{MP} 2(\mathrm{Full})-\mathrm{CCSD}(\mathrm{T})$ & 7.75 \\
\hline \multirow{5}{*}{ TS-4T } & M06-2x & -93.85 \\
\hline & MP2(Full) & -41.89 \\
\hline & CCSD(T) Single Point & -72.06 \\
\hline & $\Delta: \mathrm{M} 06-2 \mathrm{x}-\mathrm{CCSD}(\mathrm{T})$ & -21.79 \\
\hline & $\Delta: \mathrm{MP} 2(\mathrm{Full})-\mathrm{CCSD}(\mathrm{T})$ & 30.17 \\
\hline \multirow{5}{*}{$\mathrm{CHOHCH}+\mathrm{HCO}$} & M06-2x & -115.64 \\
\hline & MP2(Full) & -75.98 \\
\hline & CCSD(T) Single Point & -103.56 \\
\hline & $\Delta: \mathrm{M} 06-2 \mathrm{x}-\mathrm{CCSD}(\mathrm{T})$ & -12.08 \\
\hline & $\Delta:$ MP2(Full) - CCSD(T) & 27.58 \\
\hline
\end{tabular}

\begin{tabular}{|c|c|c|}
\hline Species & Level of Theory & Rel. E (kJ/mol) \\
\hline \multirow{5}{*}{ TS-5T } & M06-2x & \begin{tabular}{|l|}
-199.48 \\
\end{tabular} \\
\hline & MP2(Full) & -171.58 \\
\hline & CCSD(T) Single Point & -176.31 \\
\hline & $\Delta: \mathrm{M} 06-2 \mathrm{x}-\mathrm{CCSD}(\mathrm{T})$ & -23.16 \\
\hline & $\Delta: \mathrm{MP} 2(\mathrm{Full})-\mathrm{CCSD}(\mathrm{T})$ & 4.73 \\
\hline \multirow{5}{*}{$\mathrm{CH} 2 \mathrm{OHCH}+\mathrm{CO}$} & M06-2x & -253.38 \\
\hline & MP2(Full) & -199.40 \\
\hline & CCSD(T) Single Point & -194.95 \\
\hline & $\Delta: \mathrm{M} 06-2 \mathrm{x}-\mathrm{CCSD}(\mathrm{T})$ & -58.43 \\
\hline & $\Delta:$ MP2(Full) - CCSD(T) & -4.46 \\
\hline \multirow{5}{*}{ TS-6T } & M06-2x & -193.64 \\
\hline & MP2(Full) & -113.19 \\
\hline & CCSD(T) Single Point & -162.97 \\
\hline & $\Delta: \mathrm{M} 06-2 \mathrm{x}-\mathrm{CCSD}(\mathrm{T})$ & -30.67 \\
\hline & $\Delta:$ MP2(Full) - CCSD(T) & 49.78 \\
\hline \multirow{5}{*}{ IM-4T } & M06-2x & -291.03 \\
\hline & MP2(Full) & -276.16 \\
\hline & CCSD(T) Single Point & -274.36 \\
\hline & $\Delta: \mathrm{M} 06-2 \mathrm{x}-\mathrm{CCSD}(\mathrm{T})$ & -16.67 \\
\hline & $\Delta:$ MP2(Full) - CCSD(T) & -1.79 \\
\hline \multirow{5}{*}{ TS-7T } & M06-2x & -229.52 \\
\hline & MP2(Full) & -206.37 \\
\hline & CCSD(T) Single Point & -214.25 \\
\hline & $\Delta: \mathrm{M} 06-2 \mathrm{x}-\operatorname{CCSD}(\mathrm{T})$ & -15.27 \\
\hline & $\Delta:$ MP2(Full) - CCSD(T) & 7.88 \\
\hline \multirow{5}{*}{$\mathrm{CHOHCH} 2+\mathrm{CO}$} & M06-2x & -249.12 \\
\hline & MP2(Full) & -245.39 \\
\hline & CCSD(T) Single Point & -242.59 \\
\hline & $\Delta: \mathrm{M} 06-2 \mathrm{x}-\mathrm{CCSD}(\mathrm{T})$ & -6.53 \\
\hline & $\Delta: \mathrm{MP} 2(\mathrm{Full})-\mathrm{CCSD}(\mathrm{T})$ & -2.80 \\
\hline \multirow{5}{*}{ TS-8T } & M06-2x & -116.43 \\
\hline & MP2(Full) & - \\
\hline & CCSD(T) Single Point & -81.84 \\
\hline & $\Delta: \mathrm{M} 06-2 \mathrm{x}-\mathrm{CCSD}(\mathrm{T})$ & -34.59 \\
\hline & $\Delta:$ MP2(Full) - CCSD(T) & - \\
\hline \multirow{5}{*}{ IM-5T } & M06-2x & -121.39 \\
\hline & MP2(Full) & -40.38 \\
\hline & CCSD(T) Single Point & -93.06 \\
\hline & $\Delta: \mathrm{M} 06-2 \mathrm{x}-\mathrm{CCSD}(\mathrm{T})$ & -28.33 \\
\hline & $\Delta: \mathrm{MP} 2(\mathrm{Full})-\mathrm{CCSD}(\mathrm{T})$ & 52.68 \\
\hline
\end{tabular}


Table 5. Continued relative energies for structures on the triplet potential energy surface, computed using the M06-2x, MP2, and CCSD(T)//M06-2x single point methods. Relative energy differences, $\Delta$, for M06-2x and MP2 results compared to CCSD(T) single-point results are also shown.

\begin{tabular}{|c|c|c|}
\hline Species & Level of Theory & Rel. E (kJ/mol) \\
\hline \multirow{5}{*}{ TS-9T } & M06-2x & -95.27 \\
\hline & MP2(Full) & -42.51 \\
\hline & CCSD(T) Single Point & -65.78 \\
\hline & $\Delta: \mathrm{M} 06-2 \mathrm{x}-\mathrm{CCSD}(\mathrm{T})$ & -29.48 \\
\hline & $\Delta:$ MP2(Full) - CCSD(T) & 23.28 \\
\hline \multirow{5}{*}{ IM-6T } & M06-2x & -285.86 \\
\hline & MP2(Full) & -220.53 \\
\hline & CCSD(T) Single Point & -258.92 \\
\hline & $\Delta: \mathrm{M} 06-2 \mathrm{x}-\mathrm{CCSD}(\mathrm{T})$ & -26.94 \\
\hline & $\Delta:$ MP2(Full) - CCSD(T) & 38.40 \\
\hline \multirow{5}{*}{ TS-10T } & M06-2x & -130.12 \\
\hline & MP2(Full) & -72.86 \\
\hline & CCSD(T) Single Point & -108.93 \\
\hline & $\Delta: \mathrm{M} 06-2 \mathrm{x}-\operatorname{CCSD}(\mathrm{T})$ & -21.20 \\
\hline & $\Delta: \mathrm{MP} 2$ (Full) - CCSD(T) & 36.07 \\
\hline \multirow{5}{*}{$\mathrm{HCO}+\mathrm{CHCHOH}$} & M06-2x & -140.74 \\
\hline & MP2(Full) & -101.43 \\
\hline & CCSD(T) Single Point & -120.69 \\
\hline & $\Delta: \mathrm{M} 06-2 \mathrm{x}-\mathrm{CCSD}(\mathrm{T})$ & -20.05 \\
\hline & $\Delta:$ MP2(Full) - CCSD(T) & 19.25 \\
\hline \multirow{5}{*}{ TS-11T } & M06-2x & -78.51 \\
\hline & MP2(Full) & -23.55 \\
\hline & CCSD(T) Single Point & -41.61 \\
\hline & $\Delta: \mathrm{M} 06-2 \mathrm{x}-\mathrm{CCSD}(\mathrm{T})$ & -36.90 \\
\hline & $\Delta:$ MP2(Full) - CCSD(T) & 18.05 \\
\hline \multirow{5}{*}{ IM-7T } & M06-2x & -226.40 \\
\hline & MP2(Full) & -202.24 \\
\hline & CCSD(T) Single Point & -201.51 \\
\hline & $\Delta: \mathrm{M} 06-2 \mathrm{x}-\mathrm{CCSD}(\mathrm{T})$ & -24.89 \\
\hline & $\Delta: \mathrm{MP} 2($ Full) $-\mathrm{CCSD}(\mathrm{T})$ & -0.74 \\
\hline \multirow{5}{*}{ TS-12T } & M06-2x & -89.46 \\
\hline & MP2(Full) & -25.74 \\
\hline & CCSD(T) Single Point & -69.48 \\
\hline & $\Delta: \mathrm{M} 06-2 \mathrm{x}-\mathrm{CCSD}(\mathrm{T})$ & -19.97 \\
\hline & $\Delta:$ MP2(Full) - CCSD(T) & 43.74 \\
\hline \multirow{5}{*}{$\mathrm{HOC}+\mathrm{CH} 2 \mathrm{CHO}$} & M06-2x & -120.66 \\
\hline & MP2(Full) & -61.60 \\
\hline & CCSD(T) Single Point & -104.83 \\
\hline & $\Delta: \mathrm{M} 06-2 \mathrm{x}-\mathrm{CCSD}(\mathrm{T})$ & -15.83 \\
\hline & $\Delta:$ MP2(Full) - CCSD(T) & 43.23 \\
\hline
\end{tabular}

\begin{tabular}{|c|c|c|}
\hline Species & Level of Theory & Rel. E (kJ/mol) \\
\hline \multirow{5}{*}{ TS-13T } & M06-2x & \begin{tabular}{|l|}
-185.31 \\
\end{tabular} \\
\hline & MP2(Full) & - \\
\hline & CCSD(T) Single Point & -157.42 \\
\hline & $\Delta: \mathrm{M} 06-2 \mathrm{x}-\mathrm{CCSD}(\mathrm{T})$ & -27.88 \\
\hline & $\Delta: \mathrm{MP} 2(\mathrm{Full})-\mathrm{CCSD}(\mathrm{T})$ & - \\
\hline \multirow{5}{*}{ IM-8T } & M06-2x & -241.30 \\
\hline & MP2(Full) & -215.83 \\
\hline & CCSD(T) Single Point & -215.88 \\
\hline & $\Delta: M 06-2 x-\operatorname{CCSD}(\mathrm{T})$ & -25.42 \\
\hline & $\Delta: \mathrm{MP} 2(\mathrm{Full})-\mathrm{CCSD}(\mathrm{T})$ & 0.05 \\
\hline \multirow{5}{*}{ TS-14T } & M06-2x & -89.46 \\
\hline & MP2(Full) & -25.74 \\
\hline & CCSD(T) Single Point & -69.48 \\
\hline & $\Delta: \mathrm{M} 06-2 \mathrm{x}-\mathrm{CCSD}(\mathrm{T})$ & -19.97 \\
\hline & $\Delta: \mathrm{MP} 2(\mathrm{Full})-\mathrm{CCSD}(\mathrm{T})$ & 43.74 \\
\hline \multirow{5}{*}{$\mathrm{HOC}+\mathrm{CH} 2 \mathrm{CHO}$} & M06-2x & -120.66 \\
\hline & MP2(Full) & -61.60 \\
\hline & CCSD(T) Single Point & -227.29 \\
\hline & $\Delta: \mathrm{M} 06-2 \mathrm{x}-\mathrm{CCSD}(\mathrm{T})$ & 106.62 \\
\hline & $\Delta: \mathrm{MP} 2(\mathrm{Full})-\mathrm{CCSD}(\mathrm{T})$ & 165.69 \\
\hline \multirow{5}{*}{ TS-15T } & M06-2x & -141.22 \\
\hline & MP2(Full) & -83.05 \\
\hline & CCSD(T) Single Point & -114.36 \\
\hline & $\Delta: \mathrm{M} 06-2 \mathrm{x}-\mathrm{CCSD}(\mathrm{T})$ & -26.87 \\
\hline & $\Delta: \mathrm{MP} 2(\mathrm{Full})-\mathrm{CCSD}(\mathrm{T})$ & 31.31 \\
\hline \multirow{5}{*}{$\mathrm{HCO}+\mathrm{CH} 2 \mathrm{COH}$} & M06-2x & -163.17 \\
\hline & MP2(Full) & -123.12 \\
\hline & CCSD(T) Single Point & -146.39 \\
\hline & $\Delta: \mathrm{M} 06-2 \mathrm{x}-\operatorname{CCSD}(\mathrm{T})$ & -16.79 \\
\hline & $\Delta: \mathrm{MP} 2(\mathrm{Full})-\mathrm{CCSD}(\mathrm{T})$ & 23.27 \\
\hline \multirow{5}{*}{ TS-16T } & M06-2x & -27.67 \\
\hline & MP2(Full) & - \\
\hline & CCSD(T) Single Point & -1.64 \\
\hline & $\Delta: \mathrm{M} 06-2 \mathrm{x}-\mathrm{CCSD}(\mathrm{T})$ & -26.04 \\
\hline & $\Delta: \mathrm{MP} 2(\mathrm{Full})-\mathrm{CCSD}(\mathrm{T})$ & - \\
\hline \multirow{5}{*}{ IM-9T } & M06-2x & -27.50 \\
\hline & MP2(Full) & 23.29 \\
\hline & CCSD(T) Single Point & -2.18 \\
\hline & $\Delta: \mathrm{M} 06-2 \mathrm{x}-\operatorname{CCSD}(\mathrm{T})$ & -25.32 \\
\hline & $\Delta: \mathrm{MP} 2(\mathrm{Full})-\mathrm{CCSD}(\mathrm{T})$ & 25.48 \\
\hline
\end{tabular}


Table 6. Continued relative energies for structures on the triplet potential energy surface, computed using the M06-2x, MP2, and CCSD(T)//M06-2x single point methods. Relative energy differences, $\Delta$, for M06-2x and MP2 results compared to CCSD(T) single-point results are also shown.

\begin{tabular}{|c|c|c|}
\hline Species & Level of Theory & Rel. E (kJ/mol) \\
\hline \multirow{5}{*}{ *TS-17A } & M06-2x & 35.25 \\
\hline & MP2(Full) & 122.04 \\
\hline & CCSD(T) Single Point & 62.42 \\
\hline & $\Delta: \mathrm{M} 06-2 \mathrm{x}-\mathrm{CCSD}(\mathrm{T})$ & -27.17 \\
\hline & $\Delta:$ MP2(Full) - CCSD(T) & 59.61 \\
\hline \multirow{5}{*}{ *TS-17B } & M06-2x & 30.78 \\
\hline & MP2(Full) & 88.42 \\
\hline & CCSD(T) Single Point & 56.00 \\
\hline & $\Delta: \mathrm{M} 06-2 \mathrm{x}-\mathrm{CCSD}(\mathrm{T})$ & -25.23 \\
\hline & $\Delta:$ MP2(Full) - CCSD(T) & 32.42 \\
\hline \multirow{5}{*}{ TS-18T } & M06-2x & -53.10 \\
\hline & MP2(Full) & -202.82 \\
\hline & CCSD(T) Single Point & -23.10 \\
\hline & $\Delta:$ M06-2x - CCSD(T) & -30.01 \\
\hline & $\Delta:$ MP2(Full) - CCSD(T) & -179.72 \\
\hline \multirow{5}{*}{ IM-10T } & M06-2x & -126.81 \\
\hline & MP2(Full) & -44.04 \\
\hline & CCSD(T) Single Point & -100.01 \\
\hline & $\Delta: \mathrm{M} 06-2 \mathrm{x}-\mathrm{CCSD}(\mathrm{T})$ & -26.80 \\
\hline & $\Delta: \mathrm{MP} 2($ Full $)-\mathrm{CCSD}(\mathrm{T})$ & 55.97 \\
\hline \multirow{5}{*}{ TS-19T } & M06-2x & 50.33 \\
\hline & MP2(Full) & 114.90 \\
\hline & CCSD(T) Single Point & 77.39 \\
\hline & $\Delta:$ M06-2x - CCSD(T) & -27.05 \\
\hline & $\Delta: \mathrm{MP} 2($ Full $)-\mathrm{CCSD}(\mathrm{T})$ & 37.51 \\
\hline \multirow{5}{*}{ IM-11T } & M06-2x & -142.16 \\
\hline & MP2(Full) & -64.85 \\
\hline & CCSD(T) Single Point & -113.30 \\
\hline & $\Delta: \mathrm{M} 06-2 \mathrm{x}-\mathrm{CCSD}(\mathrm{T})$ & -28.86 \\
\hline & $\Delta:$ MP2(Full) - CCSD(T) & 48.44 \\
\hline \multirow{5}{*}{ TS-20T } & M06-2x & -11.91 \\
\hline & MP2(Full) & 58.90 \\
\hline & CCSD(T) Single Point & 7.16 \\
\hline & $\Delta: \mathrm{M} 06-2 \mathrm{x}-\mathrm{CCSD}(\mathrm{T})$ & -19.07 \\
\hline & $\Delta:$ MP2(Full) - CCSD(T) & 51.74 \\
\hline \multirow{5}{*}{$\mathrm{CH} 2 \mathrm{O}+\mathrm{CHCOH}$} & M06-2x & -21.09 \\
\hline & MP2(Full) & -12.11 \\
\hline & CCSD(T) Single Point & -2.36 \\
\hline & $\Delta:$ M06-2x - CCSD(T) & -18.73 \\
\hline & $\Delta:$ MP2(Full) - CCSD(T) & -9.75 \\
\hline
\end{tabular}

*Two different HOC dissociations are possible here. 
CCSD(T) single-point energies were used to construct PEPs of higher accuracy for the triplet and singlet potential energy surfaces of the propargyl alcohol reaction. The PEPs representing the established pathways (those found by Buettner) ${ }^{28}$ for the propargyl alcohol reaction at the MP2/6-31++G(d,p), M06-2x/cc-pVTZ, and CCSD(T)/cc-pVTZ//M06-2x/cc-pVTZ levels of theory are shown in Figures 15-20. The importance of collecting $\operatorname{CCSD}(\mathrm{T})$ single-point energies is revealed with these PEPs. Figure 17 shows the established HCO radical formation pathway with energies computed at the M06-2x/cc-pVTZ level of theory. As seen in Figure 17, TS-1T violates the definition of a transition state as it is lower in energy than the starting material. Figure 15 shows the PEP for the established HCO radical pathway at the higher level of theory, $\operatorname{CCSD}(\mathrm{T})$, where TS- $1 \mathrm{~T}$ is $10.40 \mathrm{~kJ} / \mathrm{mol}$ higher than the reactants, satisfying the definition of a transition state. The PEP shown in Figure 15 was generated by rerunning Buettner's MP2/6$311++\mathrm{G}(\mathrm{d}, \mathrm{p})$ structures at the M06-2x/cc-pVTZ level, followed by single-point energy calculations at the $\operatorname{CCSD}(\mathrm{T}) / \mathrm{cc}-\mathrm{pVTZ}$ level of theory. Figure 16 shows the PEP of the established singlet surface, with single-point energies calculated at the CCSD(T)/cc-pVTZ level of theory. As mentioned previously, the singlet surface does not result in the formation of HCO radical. Structures of all intermediate, transition state, and product species are included in the Appendix. 


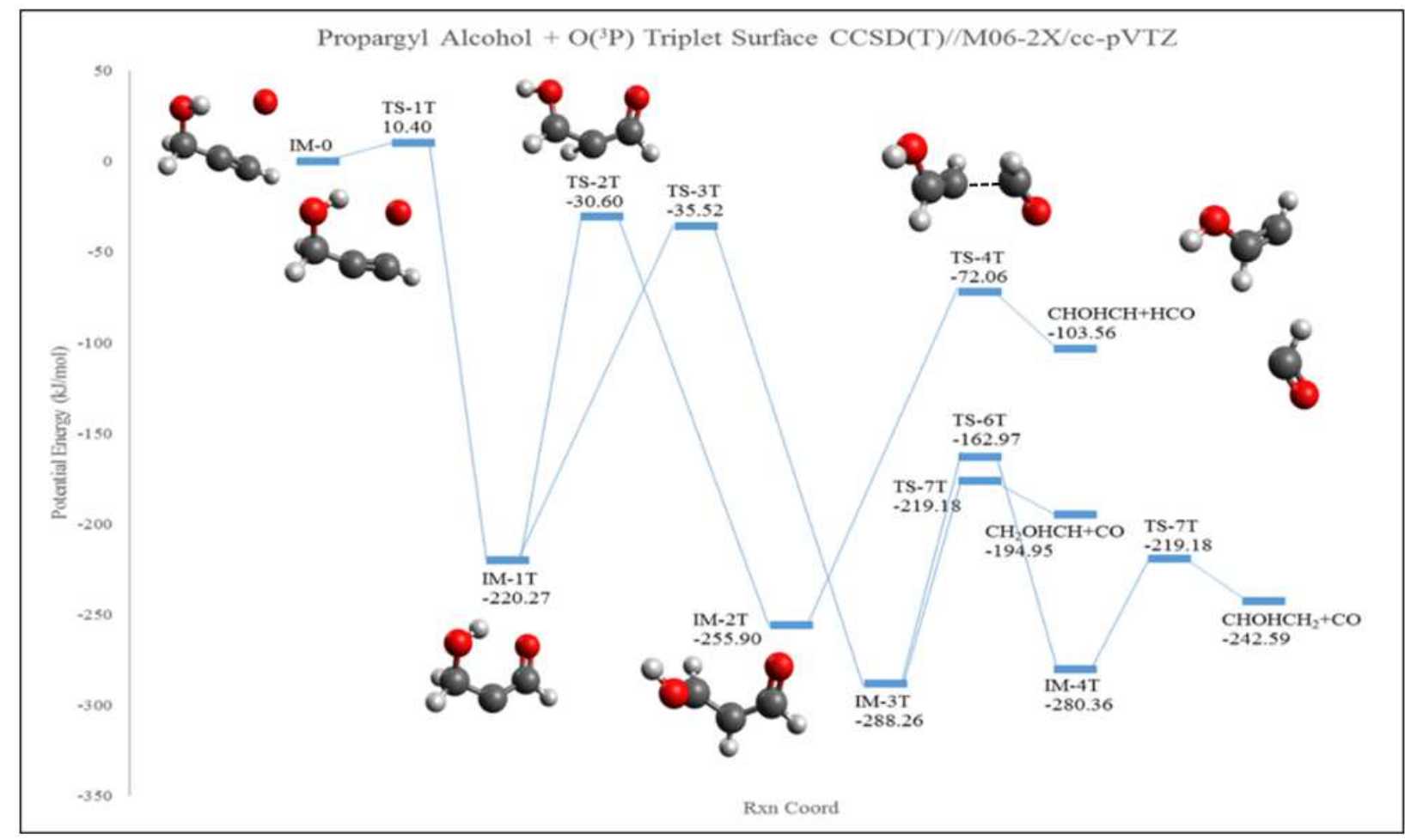

Figure 15. Established triplet surface pathways, CCSD(T)//M06-2x/cc-pVTZ level, key IM and TS structures shown.

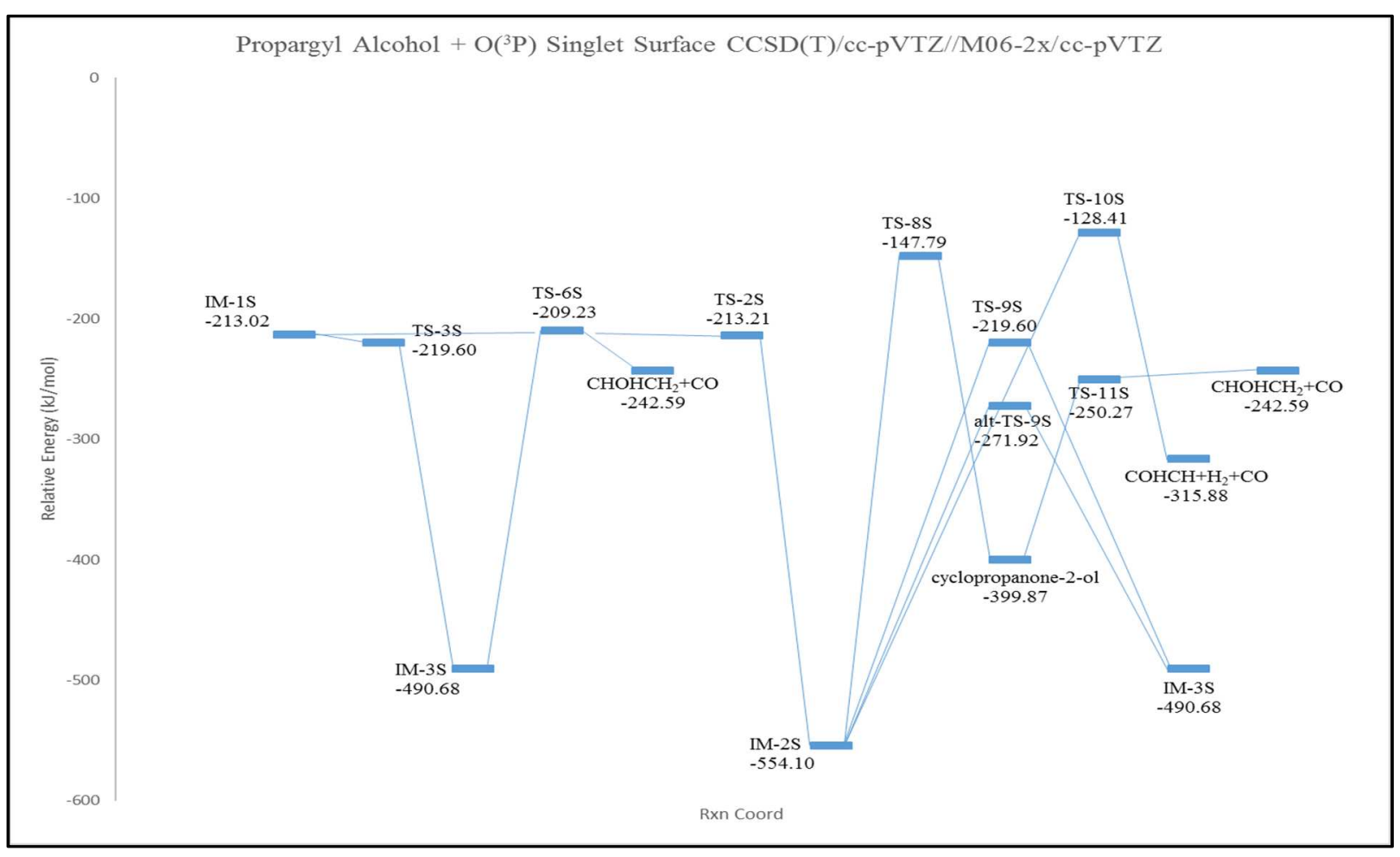

Figure 16. Established singlet surface pathways, CCSD(T)//M06-2x/cc-pVTZ level. 


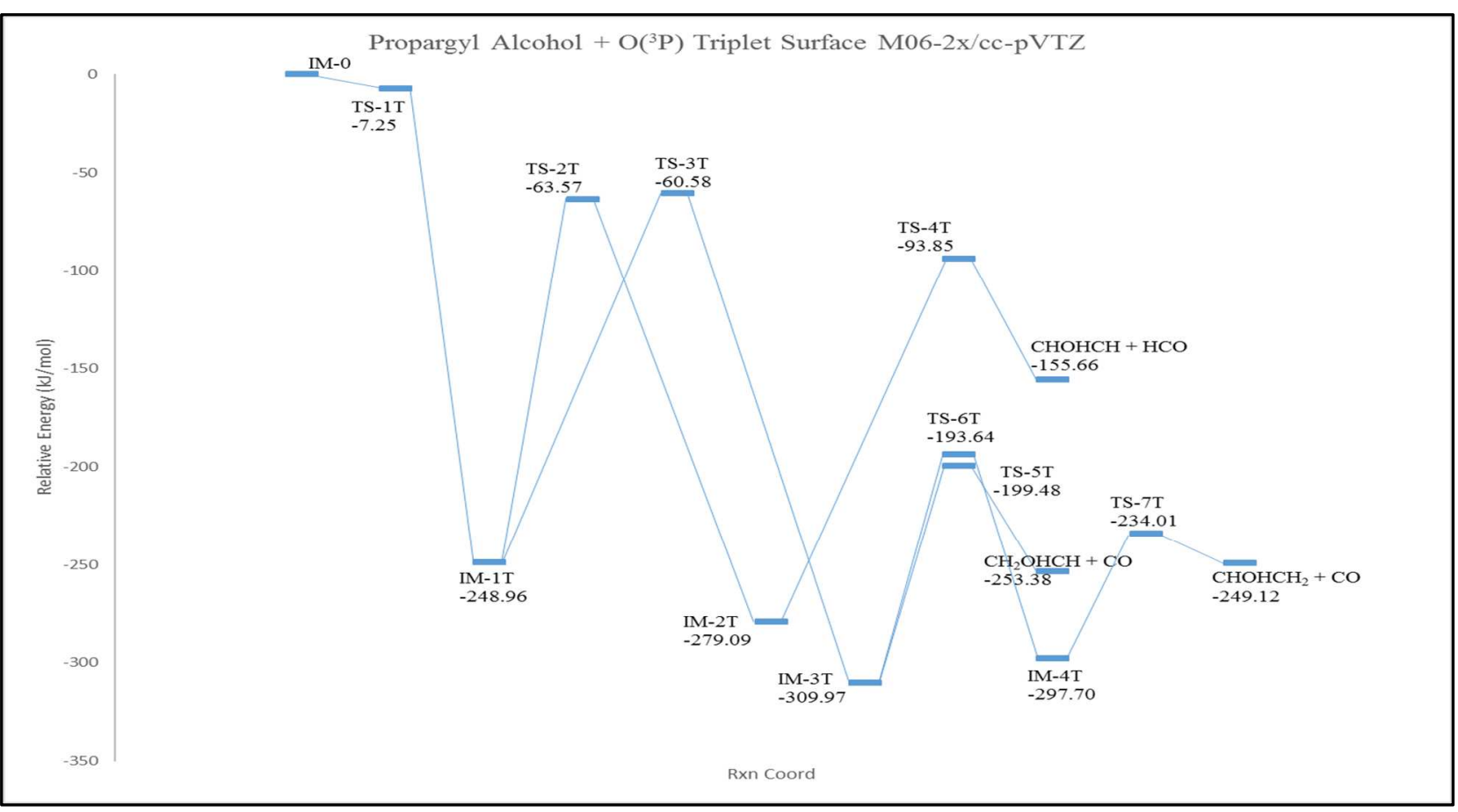

Figure 17. Established triplet surface pathways, M06-2x/cc-pVTZ level.

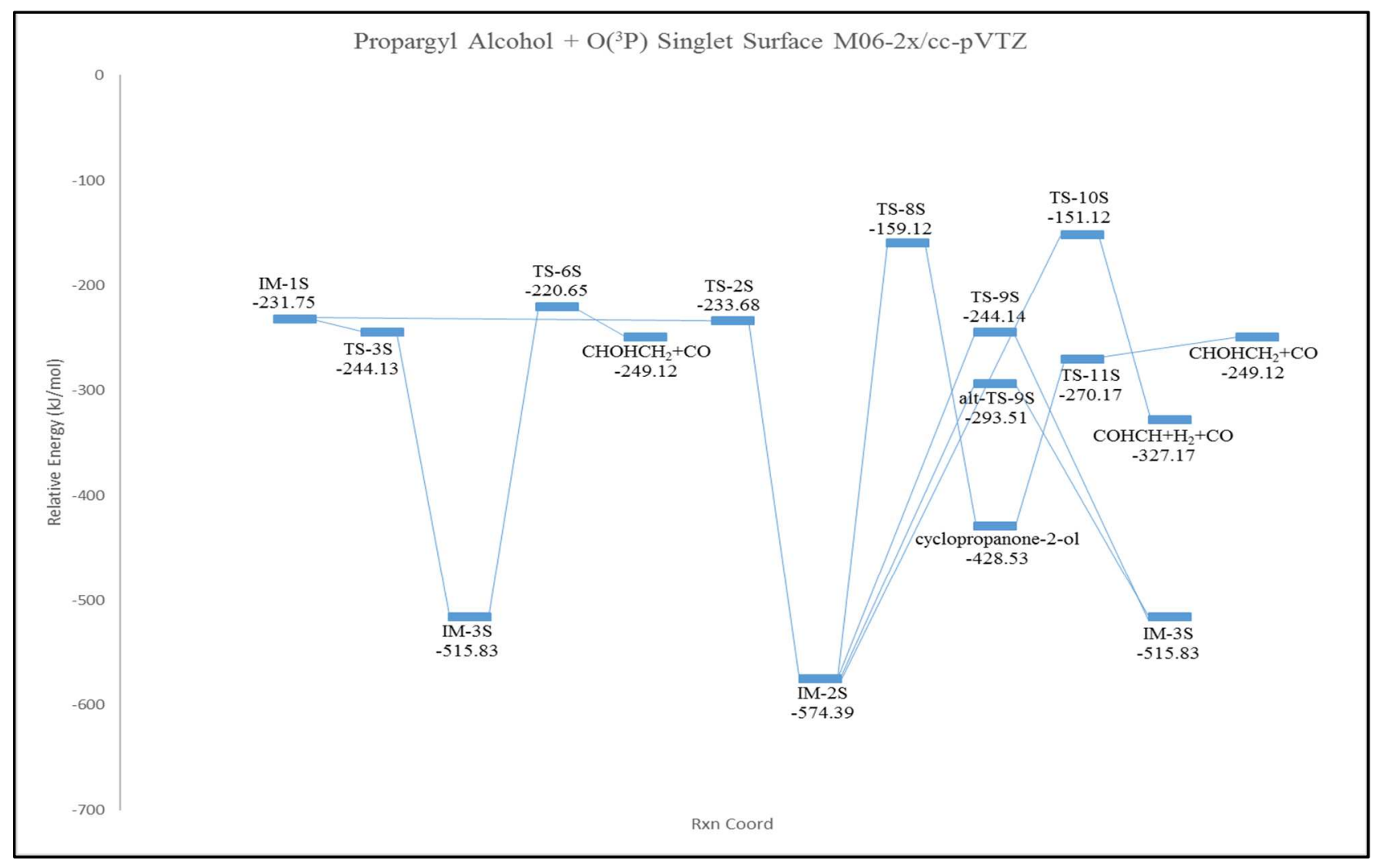

Figure 18. Established singlet surface pathways, M06-2x/cc-pVTZ level. 


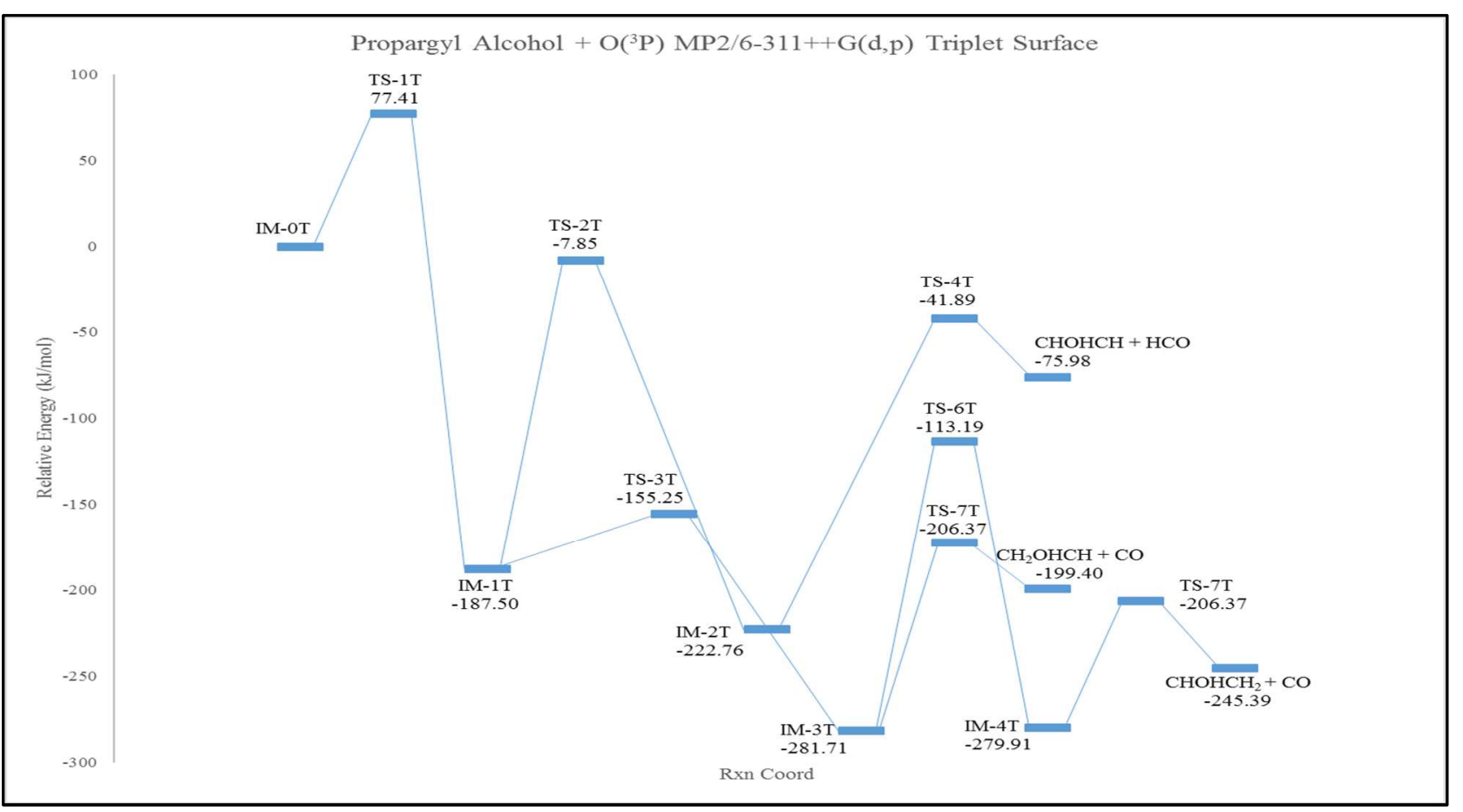

Figure 19. Established triplet surface pathways, MP2/6-311++G(d,p) level.

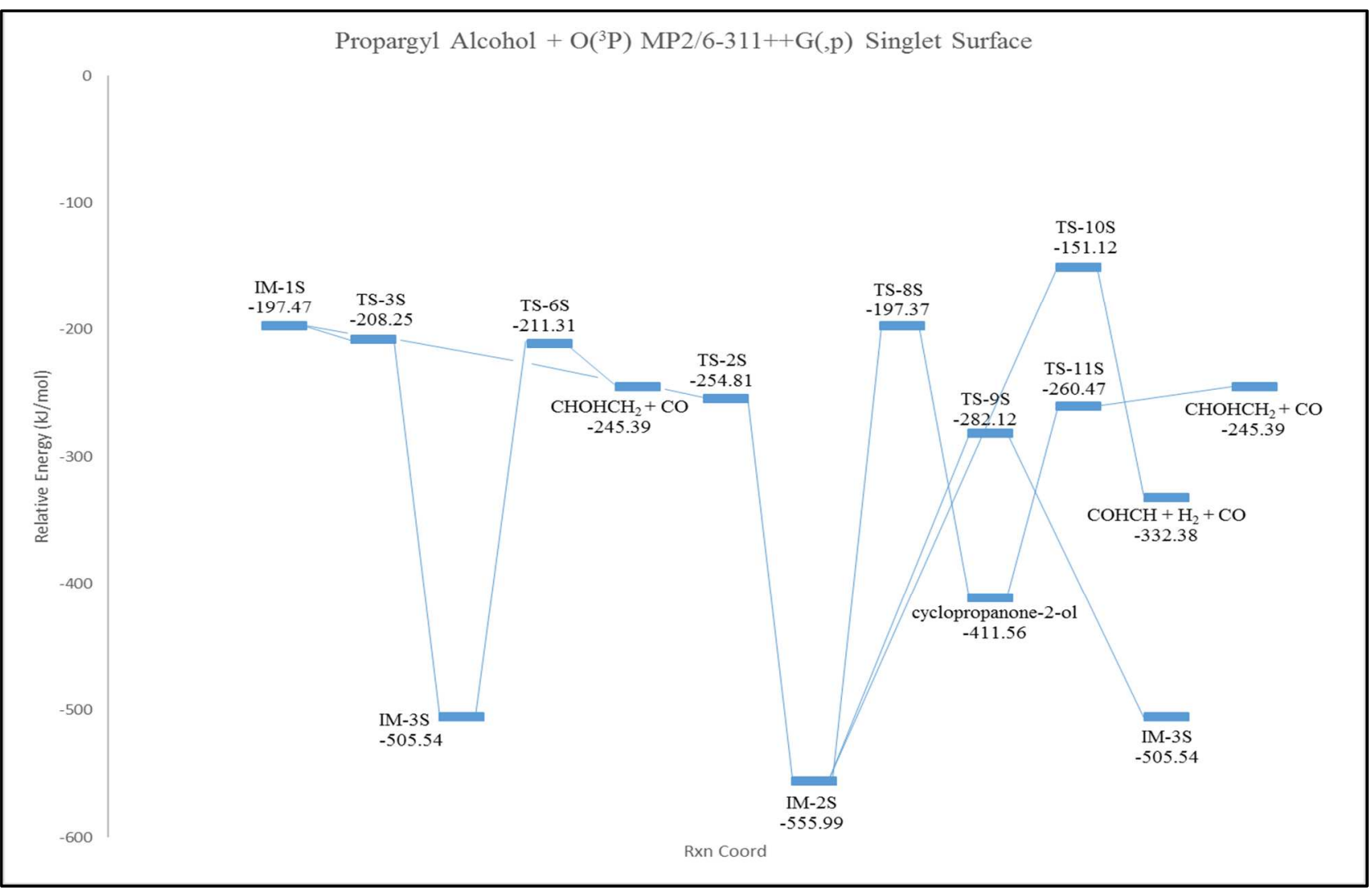

Figure 20. Established singlet surface pathways, MP2/6-311++G(d,p) level. 
Buettner found two pathways for $\mathrm{HCO}$ radical formation for the reaction of $\mathrm{O}\left({ }^{3} \mathrm{P}\right)$ with propargyl alcohol. ${ }^{28}$ This project expands upon Buettner's findings with three additional reaction pathways that result in $\mathrm{HCO}$ radical formation. The primary mechanism behind these novel $\mathrm{HCO}$ radical formation pathways is a hydrogen from the hydroxyl group, $\mathrm{O} 9 \mathrm{H} 5$, shifting to the other oxygen atom of the molecule, O8 (see Figure 14 for atom numbering). Another important mechanism at play is the isomerization of $\mathrm{HOC}$ to $\mathrm{HCO}$, making $\mathrm{HOC}$ radical formation pathways of interest. The PEP for the isomerization of the formyl radical is shown in Figure 21.

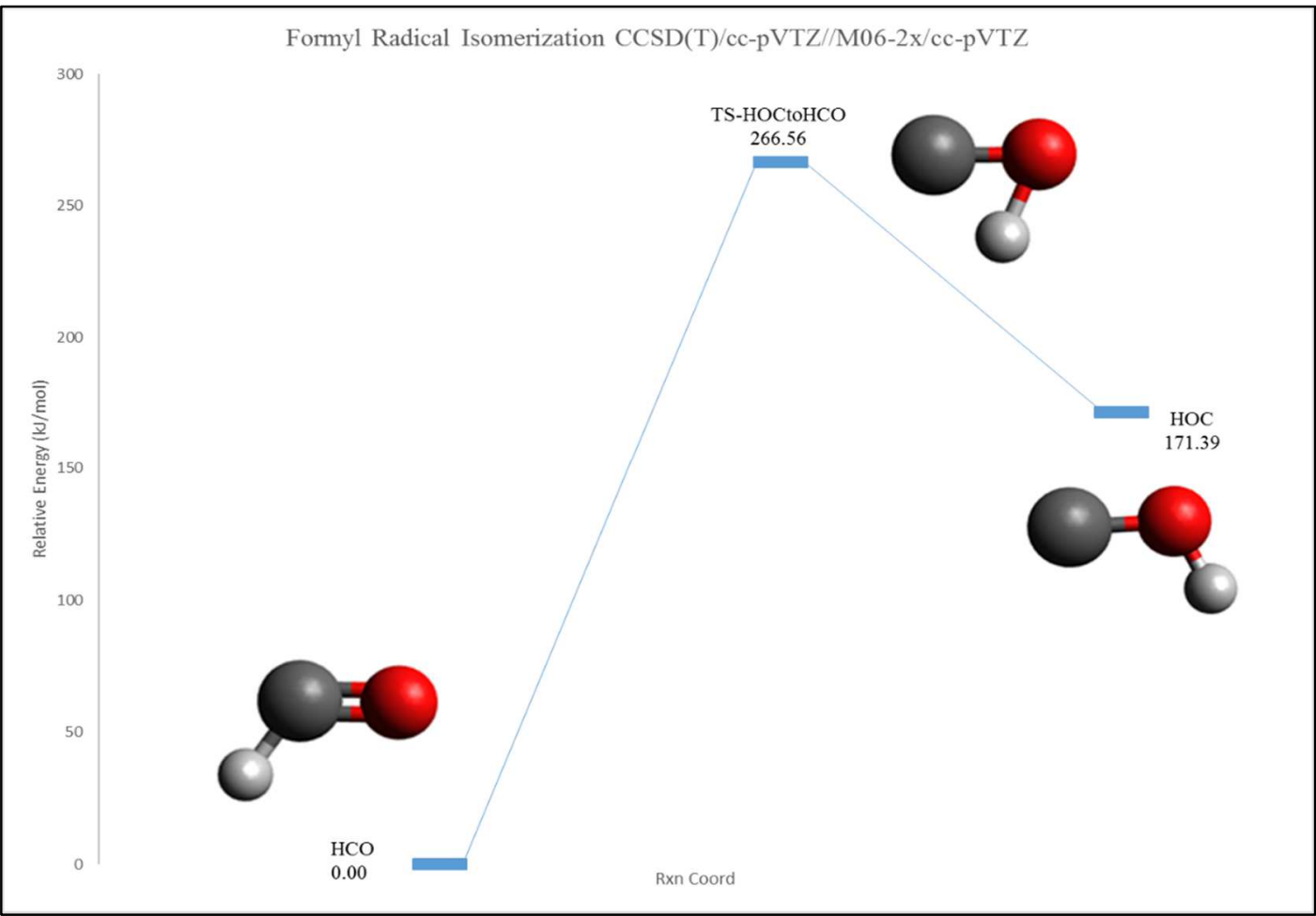

Figure 21. Isomerization of the formyl radical, computed at the $\operatorname{CCSD}(\mathrm{T}) / / \mathrm{M} 06-2 \mathrm{x} / \mathrm{cc}-\mathrm{pVTZ}$ level of theory. 
The PEPs for the novel HCO/HOC radical formation pathways are shown in Figures 22-24. At the MP2/6-311++G(d,p) level of theory, a stable structure for TS-8T could not be found, so the CCSD(T) single-point energy was used for constructing the PEP. It can also be seen that IM5T is actually higher in energy than both of its adjacent transition states (TS-8T and TS-9T). However, M06-2x performed well and yielded PEPs that were more comparable to the CCSD(T) single-point energy PEPs. The novel $\mathrm{HCO} / \mathrm{HOC}$ radical pathways presented here are predicted to be accessible due to the inherent conditions of a combustion reaction. Heats of combustion for alkyne compounds are an order of magnitude greater than the relative energy barriers present in all of the $\mathrm{HCO} / \mathrm{HOC}$ radical pathways presented here. In addition to this, a lot of excess energy from the photolysis of $\mathrm{SO}_{2}$ (the $\mathrm{O}\left({ }^{3} \mathrm{P}\right)$ source) provides the energy to overcome the relative energy barriers.

The first novel HCO radical formation pathway found in this study branches off from IM-1T in the $\mathrm{O}\left({ }^{3} \mathrm{P}\right)+$ propargyl alcohol established pathway. As seen in Figure 22, the pathway begins as $\mathrm{H} 5$ shifts from $\mathrm{O} 9$ to $\mathrm{O} 8$. The next step in the mechanism is a shift of H6 from C3 to C2. Finally, the $\mathrm{C} 2-\mathrm{C} 3$ bond breaks, resulting in the dissociation of the HCO fragment. It is worth noting that the novel HCO radical pathway that splits off from IM-1T has a lower initial relative energy (TS-8T, -81.84 kJ/mol) than the established HCO radical pathway (TS-2T, -30.60 $\mathrm{kJ} / \mathrm{mol}$ ), making the novel pathway a more thermodynamically viable pathway for HCO radical formation. The relative energies reported here are $\operatorname{CCSD}(\mathrm{T})$ single-point energies. 


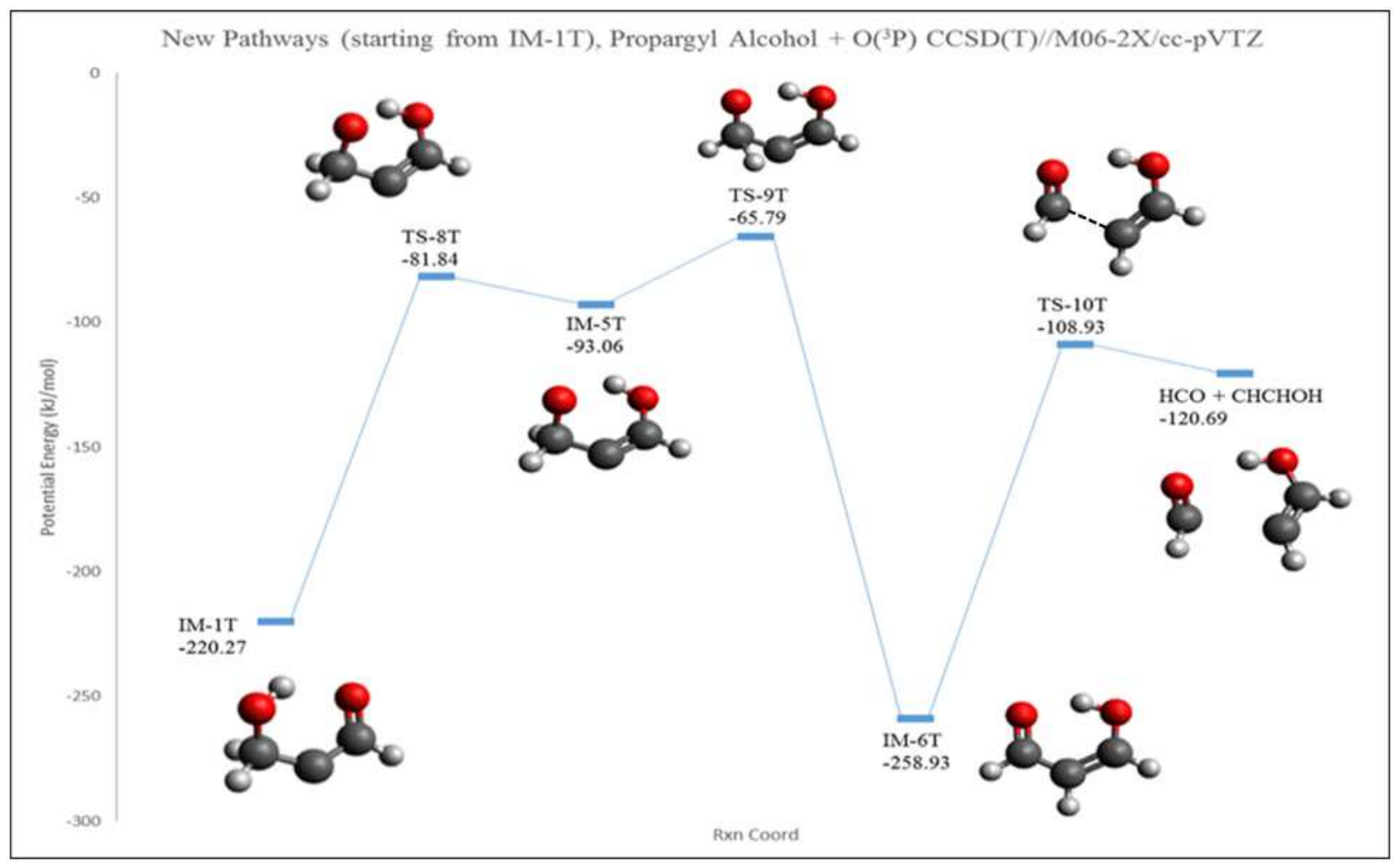

Figure 22. Novel HCO Pathway (branching off from IM-1T), CCSD(T)//M06-2x/cc-pVTZ level.

The second novel pathway for HCO radical production branches off from IM-2T. Figure 23 shows two novel pathways for the $\mathrm{O}\left({ }^{3} \mathrm{P}\right)+$ propargyl alcohol reaction: one formaldehyde formation pathway and one HOC formation pathway. The first step in the HOC pathway is an Hshift from $\mathrm{C} 3$ to $\mathrm{C} 2$. The relative energy for this transition state is $-44.51 \mathrm{~kJ} / \mathrm{mol}$ (TS-11T), which is higher in energy than the established pathway relative energy of $-72.06 \mathrm{~kJ} / \mathrm{mol}$ (TS-4T). The established pathway is predicted to be the more likely mechanism to occur once the reaction coordinate reaches IM-2T due to it being the more thermodynamically preferred pathway. 


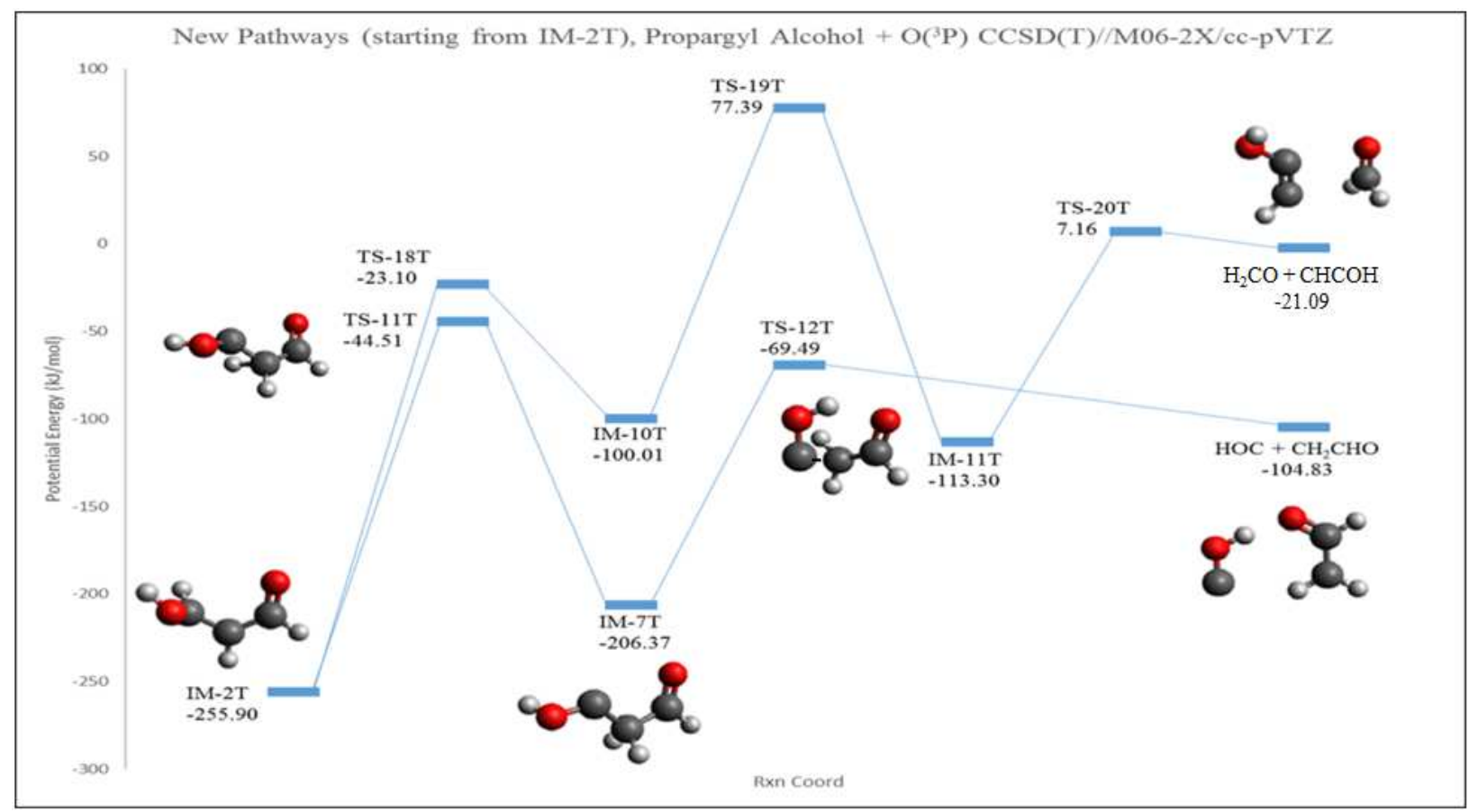

Figure 23. Novel HCO Pathway (branching off from IM-2T), CCSD(T)//M06-2x/cc-pVTZ level.

The third, fourth, and fifth novel pathways for $\mathrm{HCO} / \mathrm{HOC}$ radical production all branch off from IM-4T. Figure 24 shows the mechanisms behind the novel pathways starting from IM-4T. First, IM-4T may undergo a hydrogen shift from O9 to O8 with a relative energy of -157.42 $\mathrm{kJ} / \mathrm{mol}$ (TS-13T), which is higher in energy than TS-7T from the carbon monoxide formation channel $(-219.18 \mathrm{~kJ} / \mathrm{mol})$. However, it is worth noting that the established PEP does not show $\mathrm{HCO}$ radical formation from IM-4T and so this work provides an entirely new avenue for formation of the formyl radical. The next structure in the reaction coordinate following TS-13T is IM-8T. The dissociation of HCO radical also occurs from IM-8T via TS-14T with a relative energy of $-69.48 \mathrm{~kJ} / \mathrm{mol}$. In addition, the dissociation of HOC radical occurs at this point via TS$15 \mathrm{~T}$ with a lower relative energy of $-114.36 \mathrm{~kJ} / \mathrm{mol}$. The final novel pathway found is an HOC formation pathway that is significantly higher in energy than the other $\mathrm{HCO} / \mathrm{HOC}$ radical 
pathways. IM-4T may undergo an H-shift from $\mathrm{C} 2$ to $\mathrm{O} 8$ via TS-16T with a relative energy of $1.64 \mathrm{~kJ} / \mathrm{mol}$. The following intermediate species, IM-9T, presents two possibilities for HOC dissociation. Once one dissociation occurs, a subsequent dissociation is not possible due to the energy required to break the $\mathrm{C}=\mathrm{C}$ double bond. The relative energy for an $\mathrm{HOC}$ dissociation from IM-9T is $56.00 \mathrm{~kJ} / \mathrm{mol}$ (TS-17T). This mechanism is thermodynamically unfavorable compared to the lower relative energy barriers of other $\mathrm{HCO} / \mathrm{HOC}$ radical pathways in the $\mathrm{O}\left({ }^{3} \mathrm{P}\right)$ + propargyl alcohol reaction.

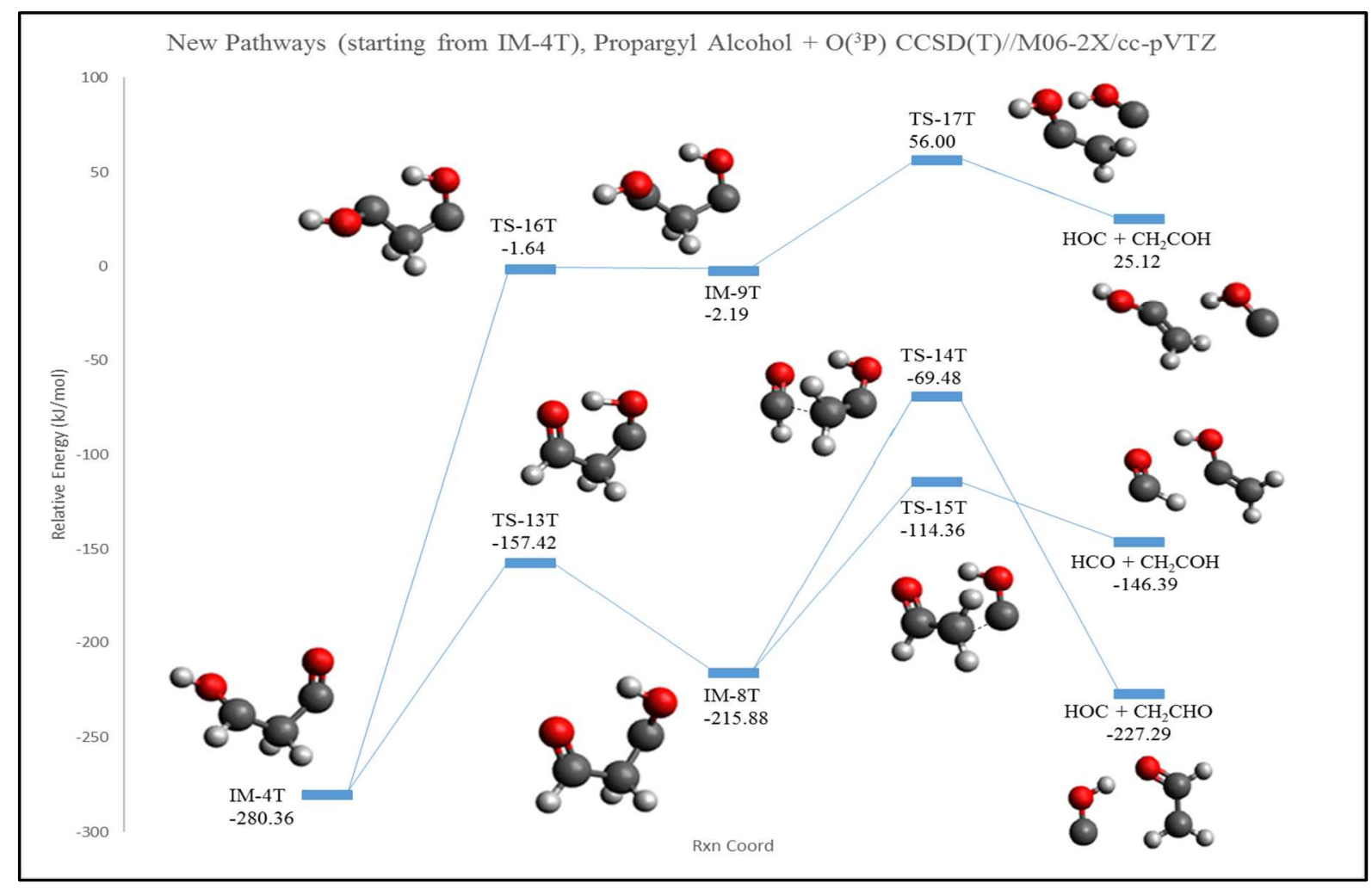

Figure 24. Novel HCO Pathway (branching off of IM-4T), CCSD(T)//M06-2x/cc-pVTZ level. 


\section{Minimum Energy Crossing Point}

Intersystem crossing occurs at the minimum energy crossing point (MECP), which was found using the MECP method by Harvey et al. ${ }^{34}$ at the M06-2x level of theory. Intersystem crossing is an important phenomenon in chemistry as it allows a system to cross from one multiplicity potential energy surface to another. The reaction of $\mathrm{O}\left({ }^{3} \mathrm{P}\right)+$ propargyl alcohol always begins on the triplet surface as the reaction is always initiated with a triplet oxygen atom attacking the triple bond of a singlet alkyne species. Similar to the $\mathrm{O}\left({ }^{3} \mathrm{P}\right)+$ propyne reaction, intersystem crossing for $\mathrm{O}\left({ }^{3} \mathrm{P}\right)+$ propargyl alcohol takes place near the first IM in the reaction pathway. HCO radical can only form on the triplet surface as shown in previous studies, ${ }^{18,28,29}$ but the singlet potential energy surface is still relevant as it is easily accessible due to a low energy crossing point structure between IM-1T and IM-1S with the potential energy difference between IM-1T and the MECP structure just $0.005 \%$. The MECP lies just above IM-1S in energy by about $13 \mathrm{~kJ} / \mathrm{mol}$, and slightly higher than IM-1T by about $37 \mathrm{~kJ} / \mathrm{mol}$. Results of the MECP method are shown in Figure 25 and table 7 . The results reveal that the geometry of the crossing-point structure is intermediate between IM-1T and IM-1S, with about half of the geometric parameters being closer to IM-1T and the other half being closer to IM-1S. 
IM-1T

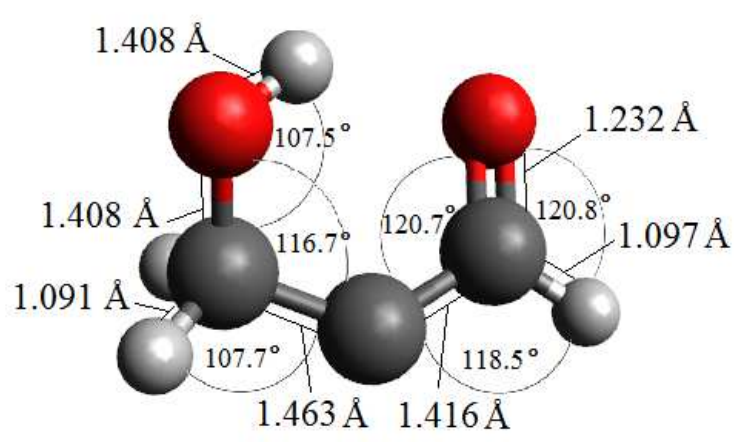

MECP

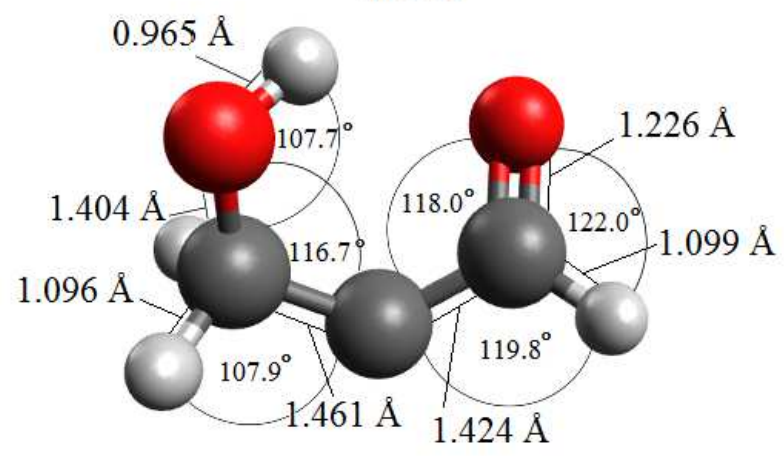

IM-1S

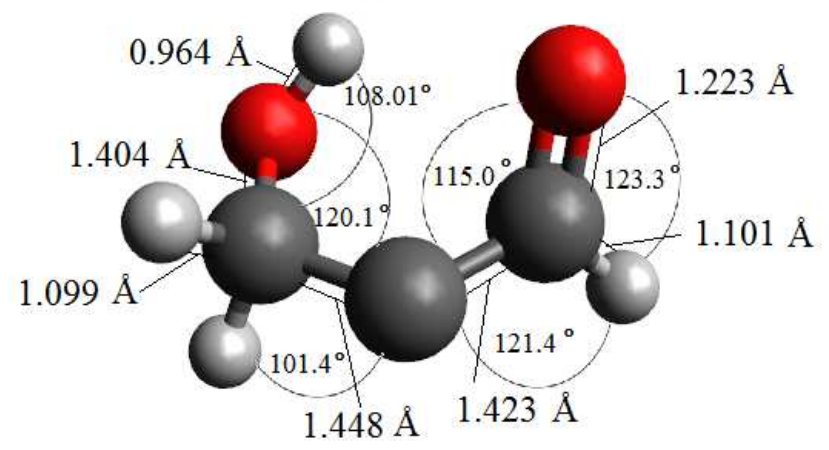

Figure 25. Structures for IM-1T (top left), MECP (top right), and IM-1S (bottom), calculated at the M06-2x/cc-pVTZ level.

Table 7. MECP results, calculated at the M06-2x/cc-pVTZ level of theory. Reported energies are not corrected for vibrational zero-point energies.

\begin{tabular}{|c|c|c|c|c|c|}
\hline \multicolumn{6}{|c|}{ MECP (M06-2x) } \\
\hline Parameter & Crossing Point Value & IM-1T & IM-1S & $\%$ Diff. (triplet) & \% Diff. (singlet) \\
\hline C1-H4 (Å) & 1.099 & 1.097 & 1.101 & -0.180 & 0.180 \\
\hline C1-C2 (Å) & 1.424 & 1.416 & 1.423 & -0.560 & -0.070 \\
\hline C1-08 (Å) & 1.226 & 1.232 & 1.223 & 0.490 & -0.240 \\
\hline C2-C3 (A) & 1.461 & 1.463 & 1.448 & 0.140 & -0.890 \\
\hline C3-09 (A) & 1.404 & 1.408 & 1.404 & 0.290 & 0.000 \\
\hline O9-H5 (Å) & 0.965 & 0.966 & 0.964 & 0.100 & -0.100 \\
\hline$<08-\mathrm{C} 1-\mathrm{H} 4\left(^{\circ}\right)$ & 122.0 & 120.8 & 123.3 & -1.0 & 1.1 \\
\hline$<08-\mathrm{C} 1-\mathrm{C} 2\left(^{\circ}\right)$ & 118.0 & 120.7 & 115.0 & 2.3 & -2.5 \\
\hline$<\mathrm{C} 1-\mathrm{C} 2-\mathrm{C} 3\left(^{\circ}\right)$ & 124.5 & 129.7 & 117.7 & 4.2 & -5.5 \\
\hline$<\mathrm{C2}-\mathrm{C3}-\mathrm{O} 9\left(^{\circ}\right)$ & 116.7 & 112.1 & 120.1 & -3.9 & 2.9 \\
\hline$<$ C3-O9-H5 $\left({ }^{\circ}\right)$ & 107.7 & 107.5 & \begin{tabular}{|l|}
108.1 \\
\end{tabular} & -0.2 & 0.4 \\
\hline Potential Energy (hartrees) & -267.0060 & -267.0200 & -267.0111 & 0.0052 & 0.0019 \\
\hline
\end{tabular}




\section{NRT Analysis: $\mathbf{O}\left({ }^{3} \mathbf{P}\right)+$ Propargyl Alcohol}

Natural Resonance Theory (NRT) $)^{36-40}$ analyses have been carried out on each stationary point in the $\mathrm{O}\left({ }^{3} \mathrm{P}\right)+$ propargyl alcohol triplet state PEP in order to better understand how the movement of electron density dictates the reaction mechanisms at play. Natural spin density (NSD) and natural bond order (NBO) data are presented in Figures 26-37. For the sake of clarity, only the $\mathrm{C}$ and $\mathrm{O}$ atoms of the reaction are shown on the NSD and NBO plots, as the $\mathrm{H}$ atoms carry relatively low NSD values in the system. Natural spin densities are calculated as the difference between $\alpha$ spin state and $\beta$ spin state populations on each atom. The NSD data for the established $\mathrm{O}\left({ }^{3} \mathrm{P}\right)+$ propargyl alcohol pathway is shown in Figure 26, where the HCO radical product is composed of $\mathrm{C} 1, \mathrm{O} 8$, and $\mathrm{H} 4$. 


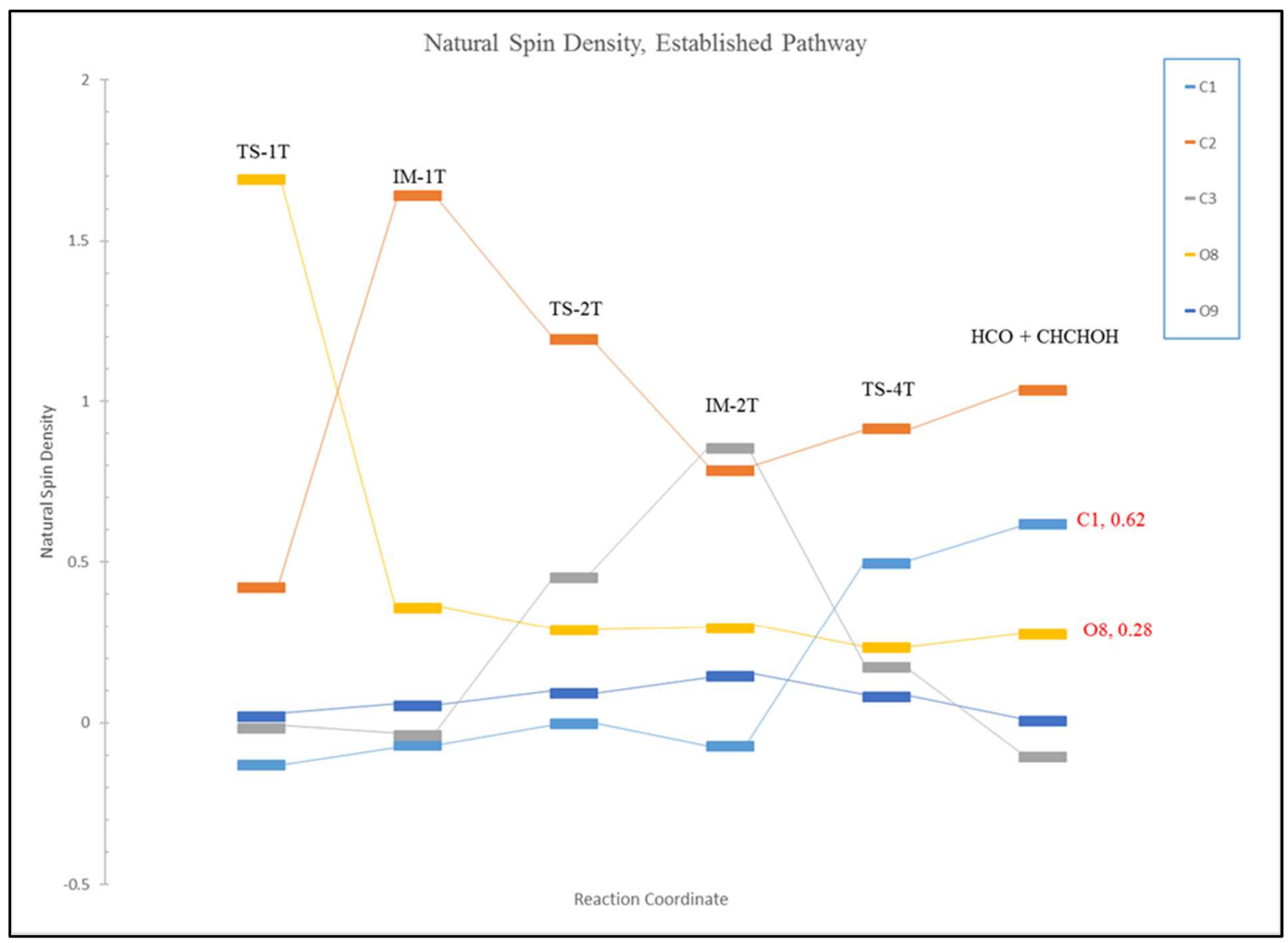

Figure 26. NSD Data for the Established HCO Pathway, calculated at the M06-2x/cc-pVTZ level of theory.

Figure 26 exemplifies the importance of conducting an NSD analysis as it reveals how the electron population shifts and confirms the dissociation of the radical species. For example, the $\mathrm{HCO} / \mathrm{HOC}$ radical product should have a total NSD of 1 as it is a radical species and bears a single unpaired electron. Figure 26 shows that $\mathrm{C} 1$ has a NSD of 0.62 and $\mathrm{O} 8$ has a NSD of 0.28 at the end of the reaction coordinate, where formyl radical dissociation occurs. The total NSD of $\mathrm{C} 1$ and $\mathrm{O} 8$ is 0.90 , with the remaining $0.10 \mathrm{NSD}$ on $\mathrm{H} 4$ giving a total NSD of 1.0 for H4 C1 O8. The NSD also shows why HCO dissociation does not occur directly from IM-1T: the spin distribution would not allow for the production of a radical at that point in the reaction pathway. 
The NBO analysis, shown in Figure 27, also reveals important information as the bond orders of the system change drastically as the reaction progresses. Figure 27 shows that the $\mathrm{C} 1-\mathrm{C} 2$ bond order starts at 1.5 at the beginning of the established HCO radical pathway and ends at 0 as it is the $\mathrm{C} 1-\mathrm{C} 2$ bond that breaks during HCO dissociation. Similar NSD and NBO patterns are observed across all novel $\mathrm{HCO} / \mathrm{HOC}$ radical pathways presented here.

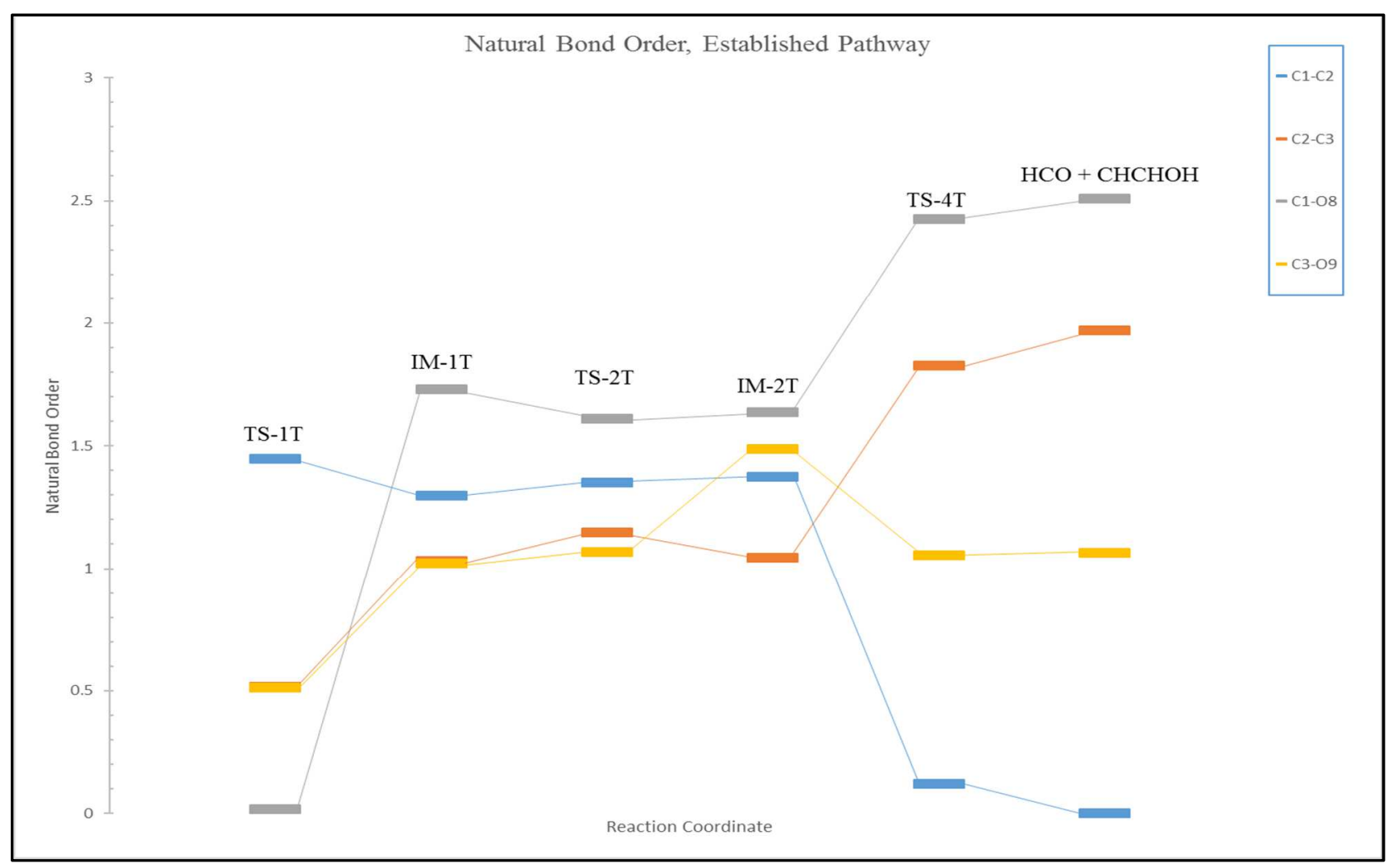

Figure 27. NBO Data for the Established HCO Pathway, calculated at the M06-2x/cc-pVTZ level of theory.

Figure 28 reveals a different shift in spin density in the $\mathrm{O}\left({ }^{3} \mathrm{P}\right)+$ propargyl alcohol system. For the first novel pathway (from IM-1T), NSD will build up on different atoms in the system 
which ultimately leads to $\mathrm{HCO}$ dissociation. For this pathway, $\mathrm{C} 3$ and $\mathrm{O} 9$ make up the $\mathrm{HCO}$ radical and have a total NSD of 0.87 , with the remaining 0.13 NSD on H7. This novel pathway involves breaking of the $\mathrm{C} 2-\mathrm{C} 3$ bond, a novel mechanism for the $\mathrm{O}\left({ }^{3} \mathrm{P}\right)+$ propargyl alcohol reaction shown in Figure 29. At the beginning of this pathway, IM-1T exhibits a bond order of 1 between $\mathrm{C} 2$ and $\mathrm{C} 3$ that ultimately falls to 0 in the dissociation step of the pathway.

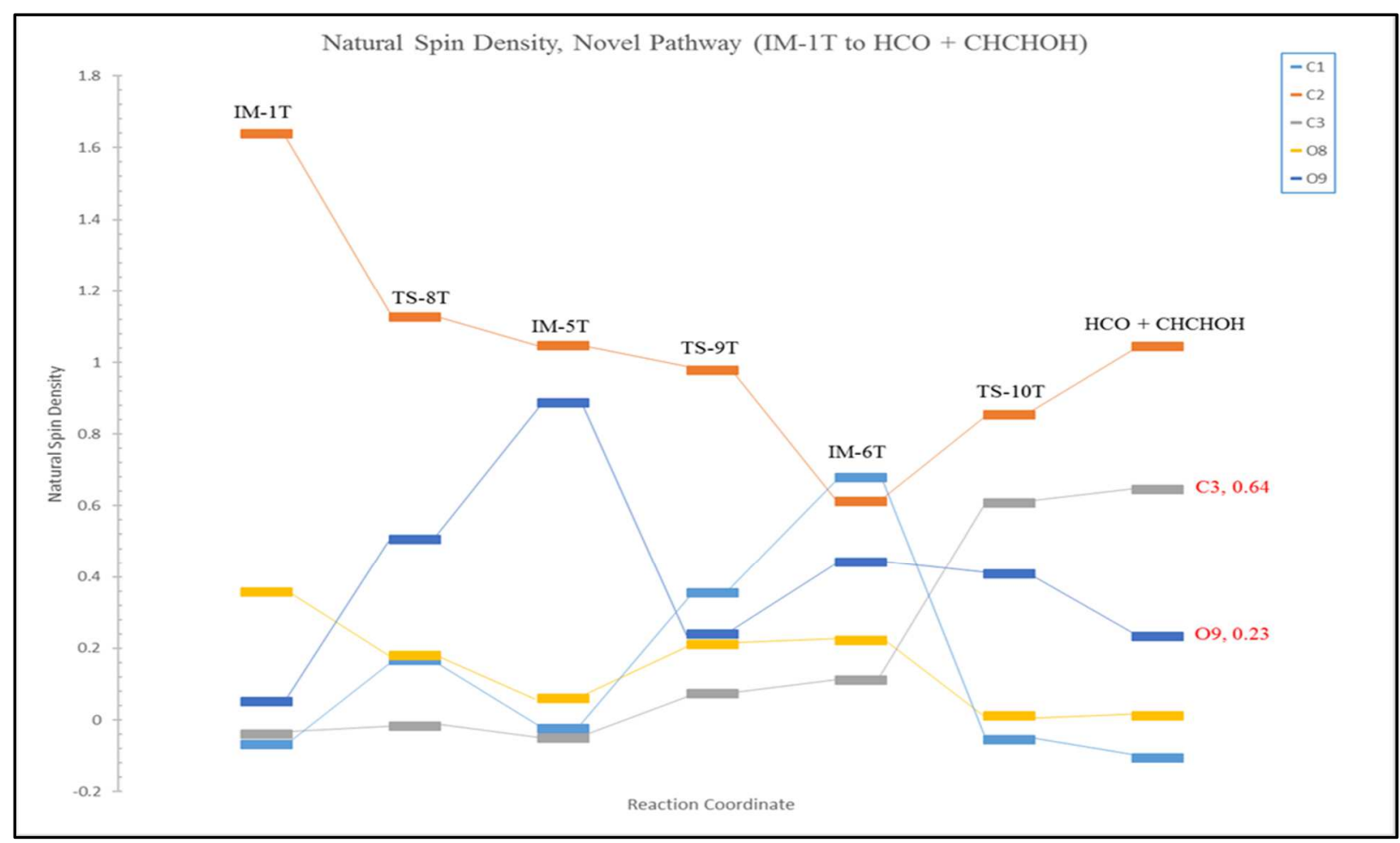

Figure 28. NSD Data for a novel HCO Pathway (IM-1T), calculated at the M06-2x/cc-pVTZ level of theory. 


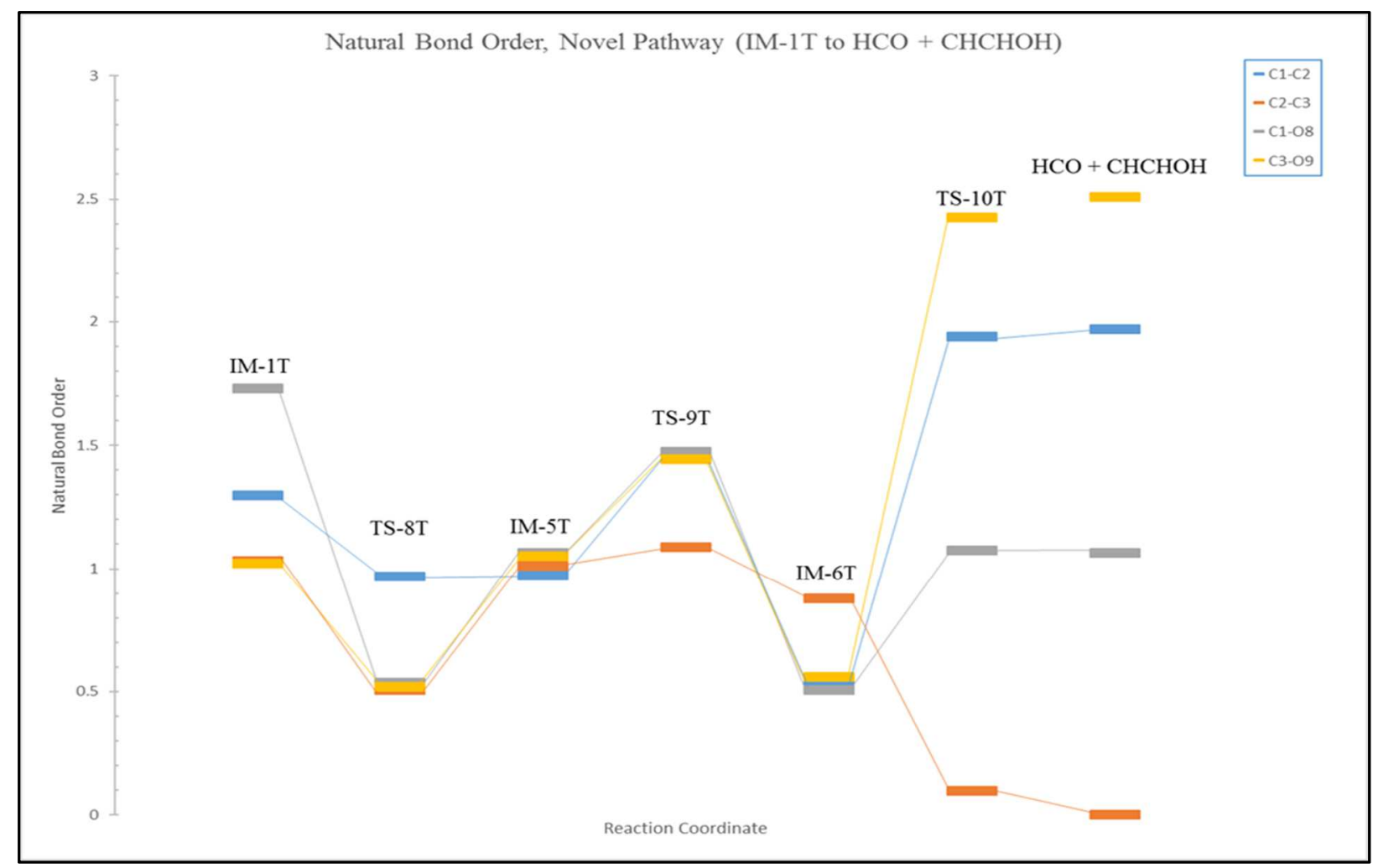

Figure 29. NBO Data for a novel HCO Pathway (IM-1T), calculated at the M06-2x/cc-pVTZ level of theory.

Different patterns in NSD are observed between HCO and HOC radical formation pathways. Generally, NSD is split across the $\mathrm{C}$ and $\mathrm{O}$ atoms of $\mathrm{HCO}$ radical in an approximately 1:3 (C:O) ratio. However, HOC formation pathways indicate something very different at play. The NSD distribution shows the unpaired electron is almost entirely located on the $\mathrm{C}$ atom in all HOC formation pathways as illustrated in Figure 30. 


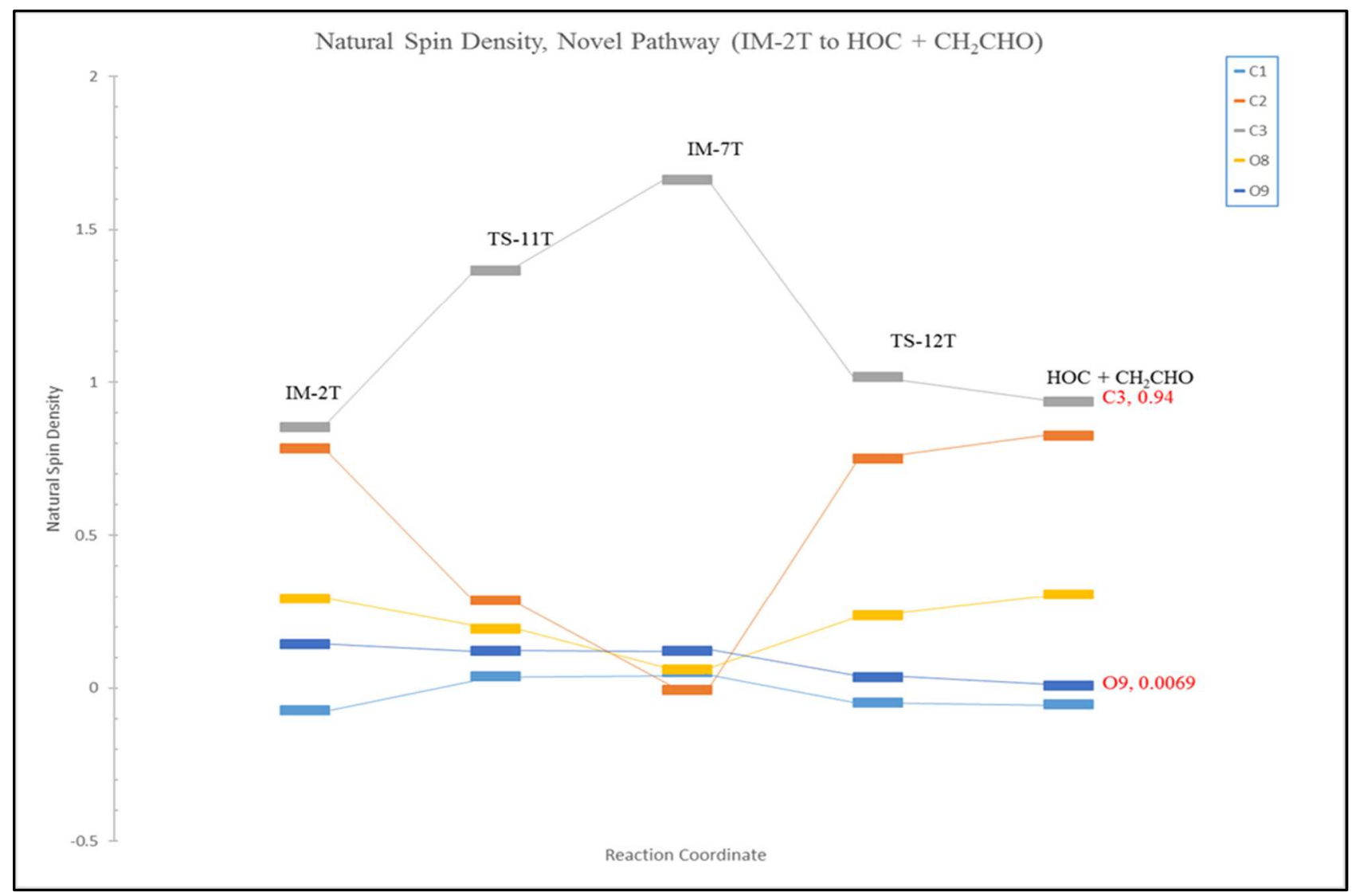

Figure 30. NSD Data for a novel HOC Pathway (IM-2T), calculated at the M06-2x/cc-pVTZ level of theory.

NBO data for the novel HOC pathway branching from IM-2T is shown in Figure 31. Again, the breaking of the $\mathrm{C} 2-\mathrm{C} 3$ bond is the key mechanism behind HOC dissociation in this pathway. NBO analysis for these novel pathways reveal that $\mathrm{HCO} / \mathrm{HOC}$ dissociation may occur from either side of the molecule, as the $\mathrm{HCO} / \mathrm{HOC}$ radical product may contain either the incoming $\mathrm{O}\left({ }^{3} \mathrm{P}\right)$ atom from the first step in the pathway or the $\mathrm{O}$ atom from the hydroxyl substituent of propargyl alcohol. 


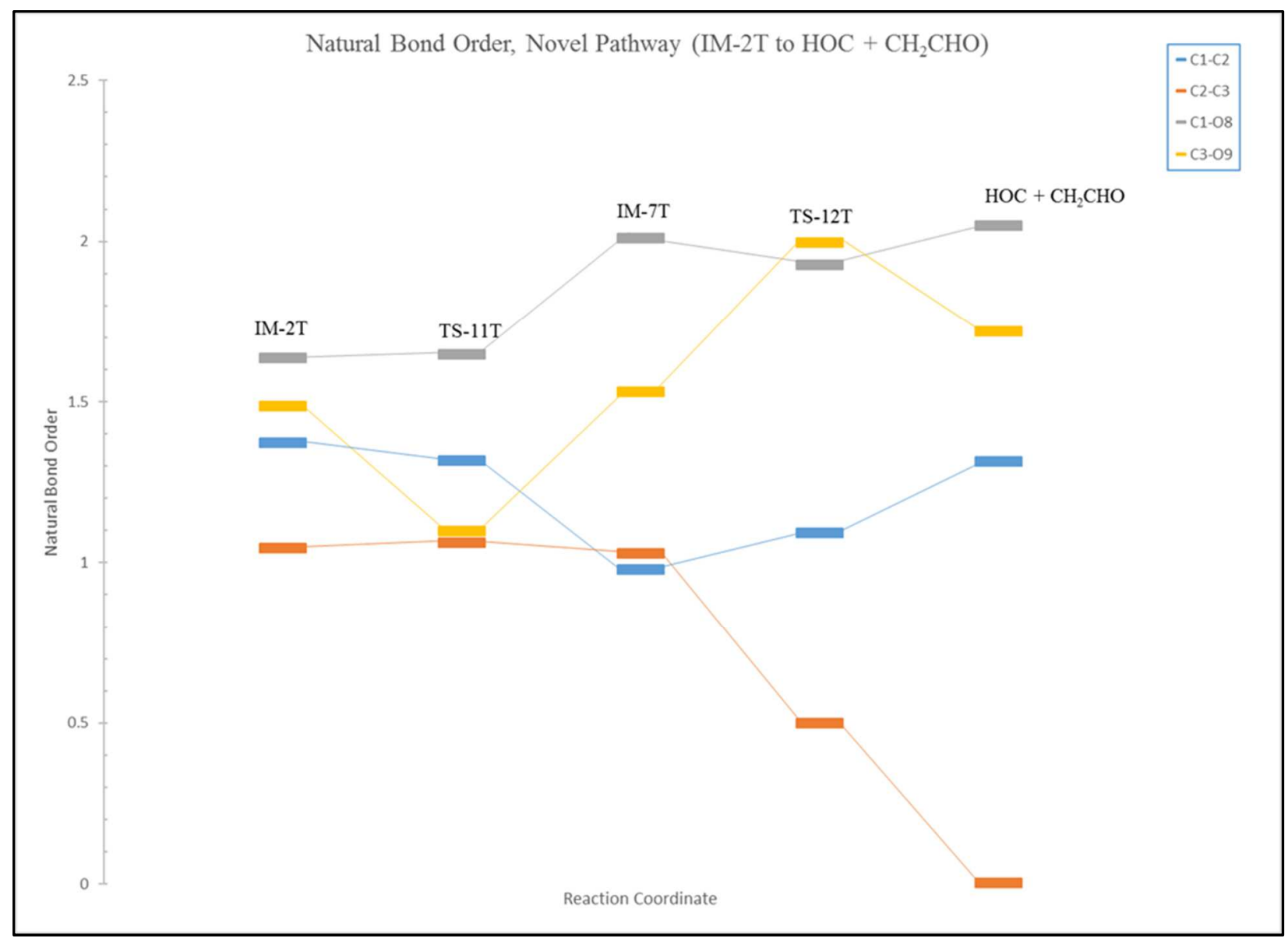

Figure 31. NBO Data for a novel HOC Pathway (IM-2T), calculated at the M06-2x/cc-pVTZ level of theory.

For completion, the NSD and NBO plots for the three novel $\mathrm{HCO} / \mathrm{HOC}$ radical pathways that branch off of IM-4T are shown in Figures 32-37. In summary, the NSD plots reveal similar patterns as to what has been presented in Figures 26-31 so far. HOC dissociations involve almost all of the NSD aggregating around the $\mathrm{C}$ atom of the HOC, whereas the HCO dissociation reveals a 1:3 ratio $(\mathrm{O}: \mathrm{C})$ for NSD. Again, the NBO analysis confirms bond breakage for the dissociation steps in the reaction pathway. 


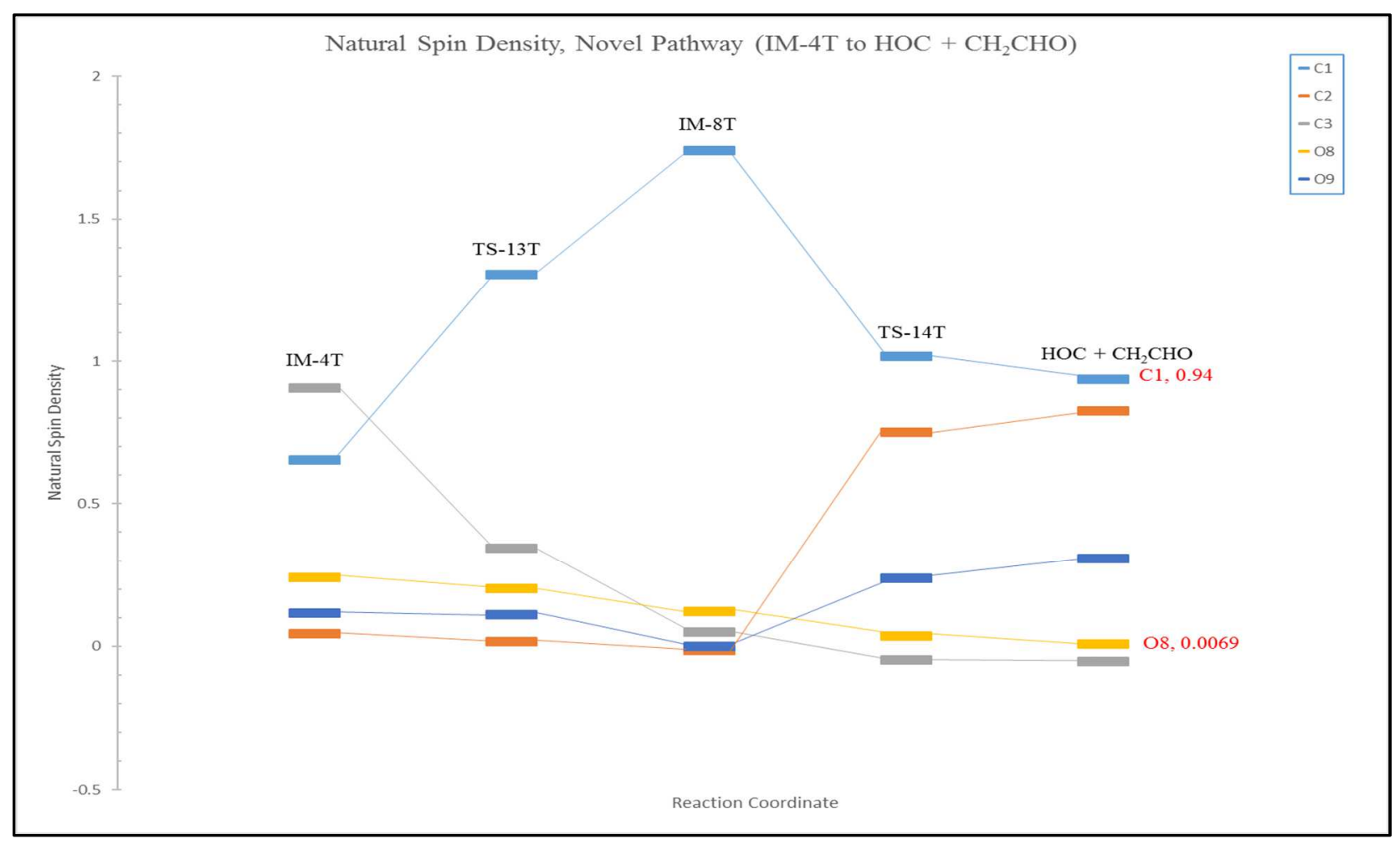

Figure 32. NSD Data for a novel HOC Pathway (IM-4T), calculated at the M06-2x/cc-pVTZ level of theory.

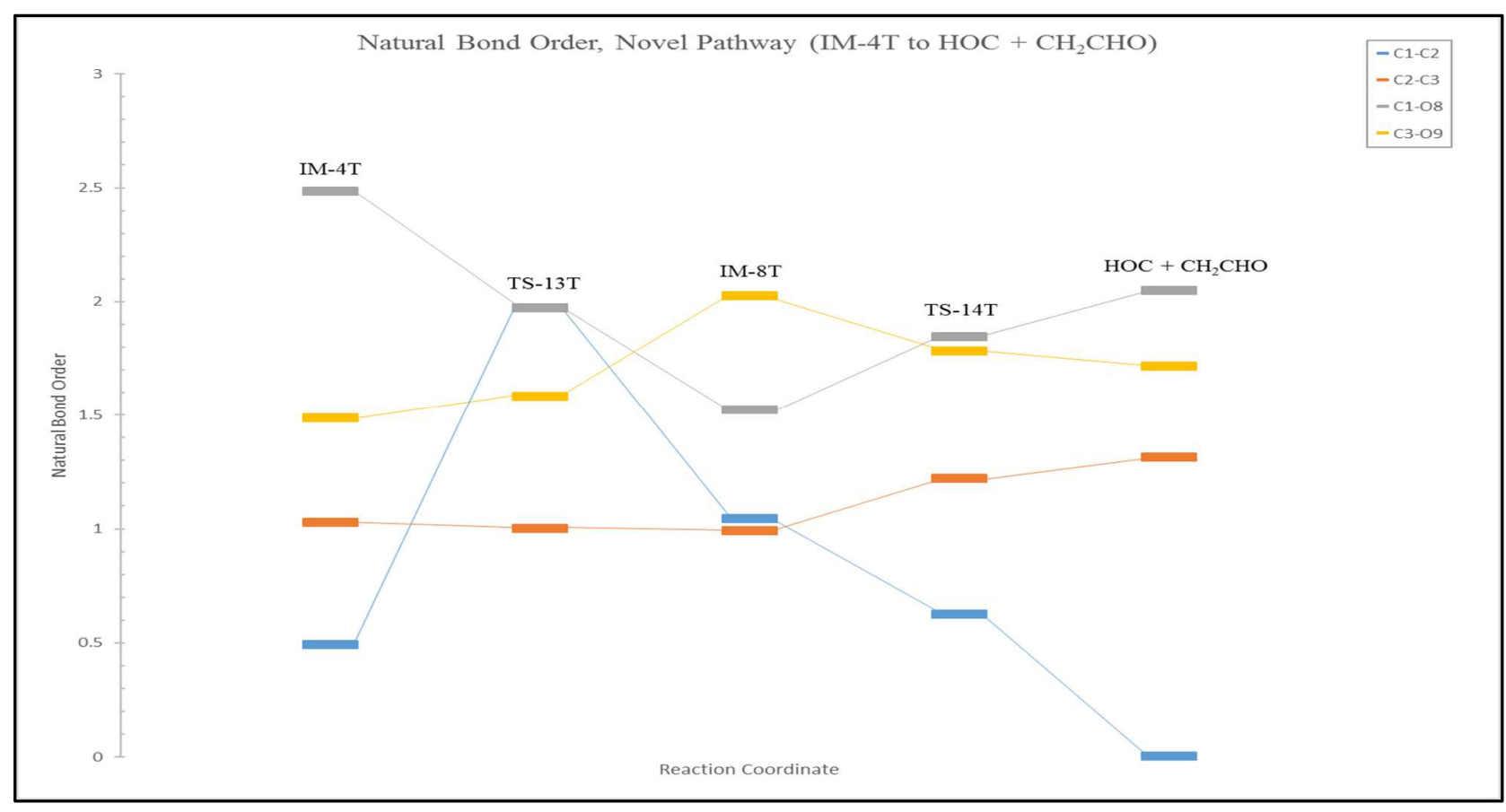

Figure 33. NBO Data for a novel HOC Pathway (IM-4T), calculated at the M06-2x/cc-pVTZ level of theory. 


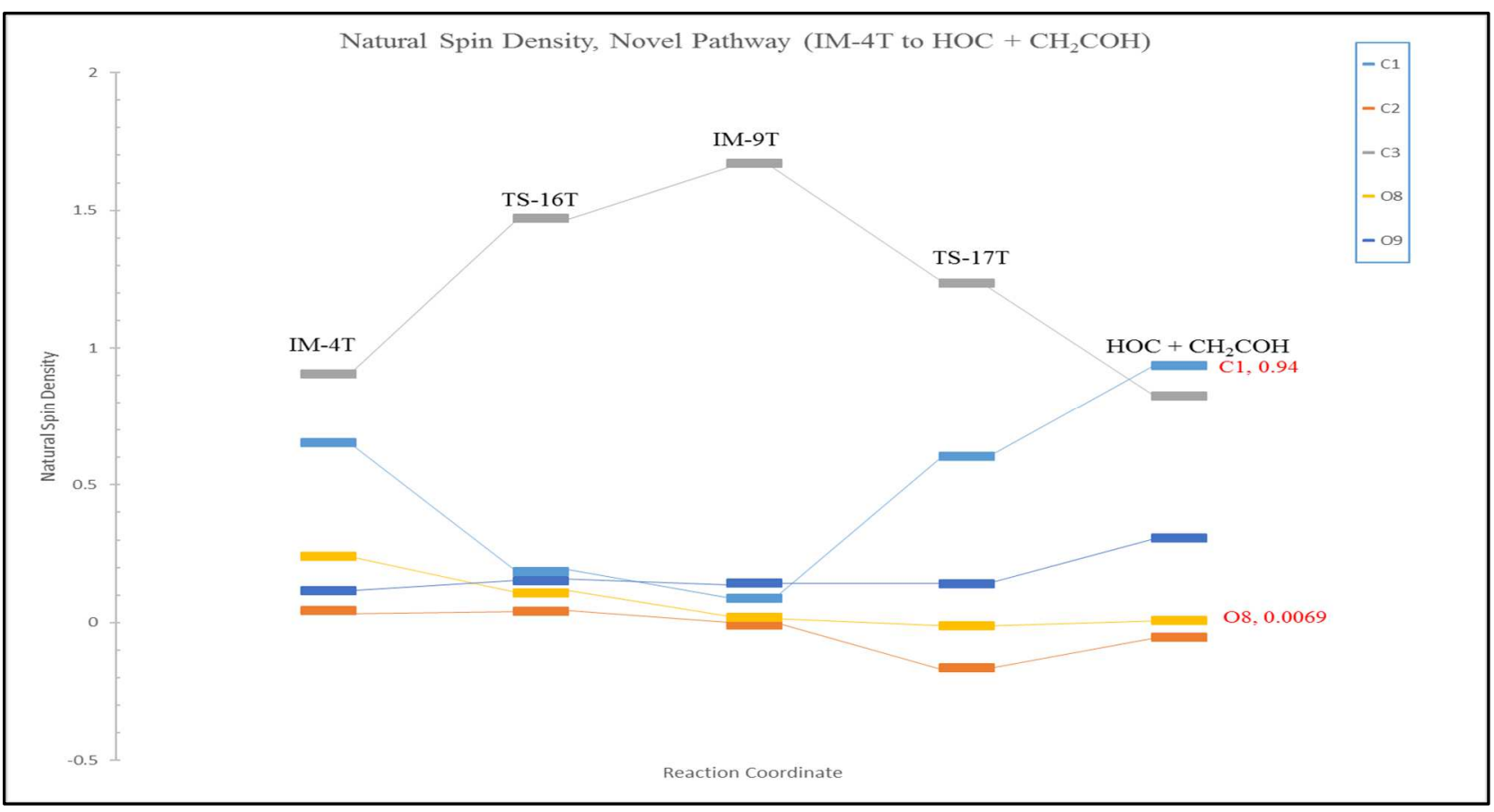

Figure 34. NSD Data for a novel HOC Pathway (IM-4T), calculated at the M06-2x/cc-pVTZ level of theory.

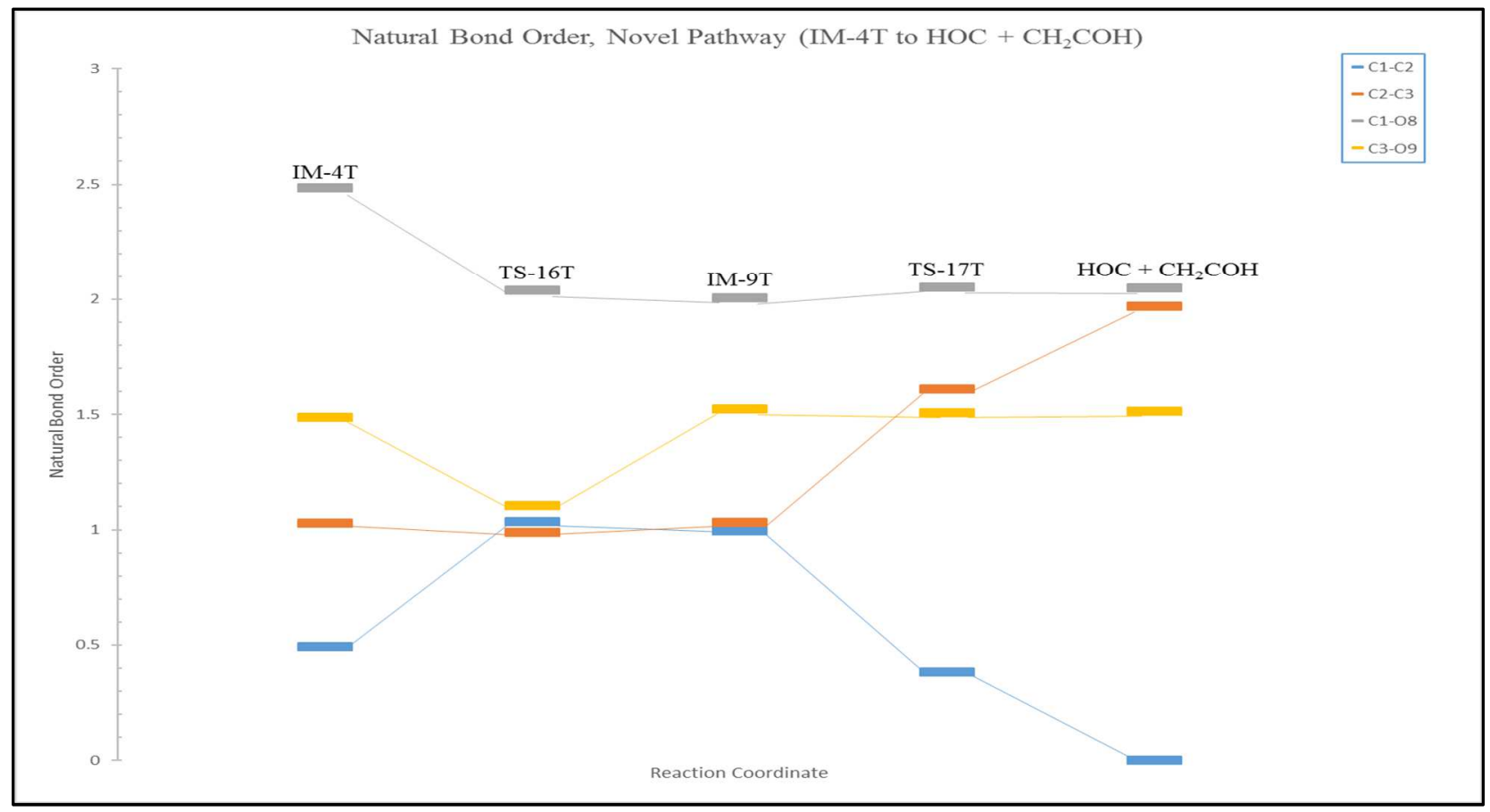

Figure 35. NBO Data for a novel HOC Pathway (IM-4T), calculated at the M06-2x/cc-pVTZ level of theory. 


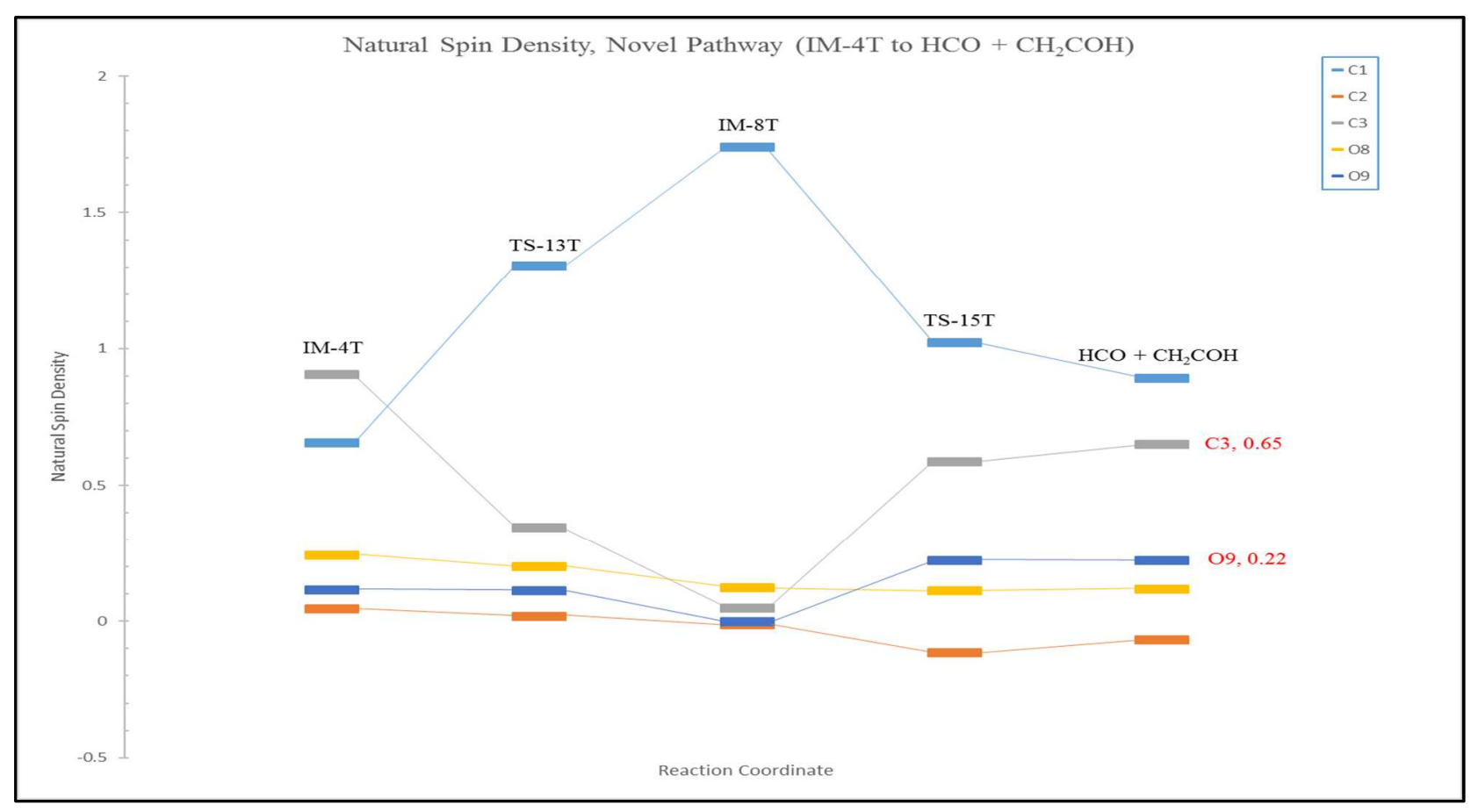

Figure 36. NSD Data for a novel HCO Pathway (IM-4T), calculated at the M06-2x/cc-pVTZ level of theory.

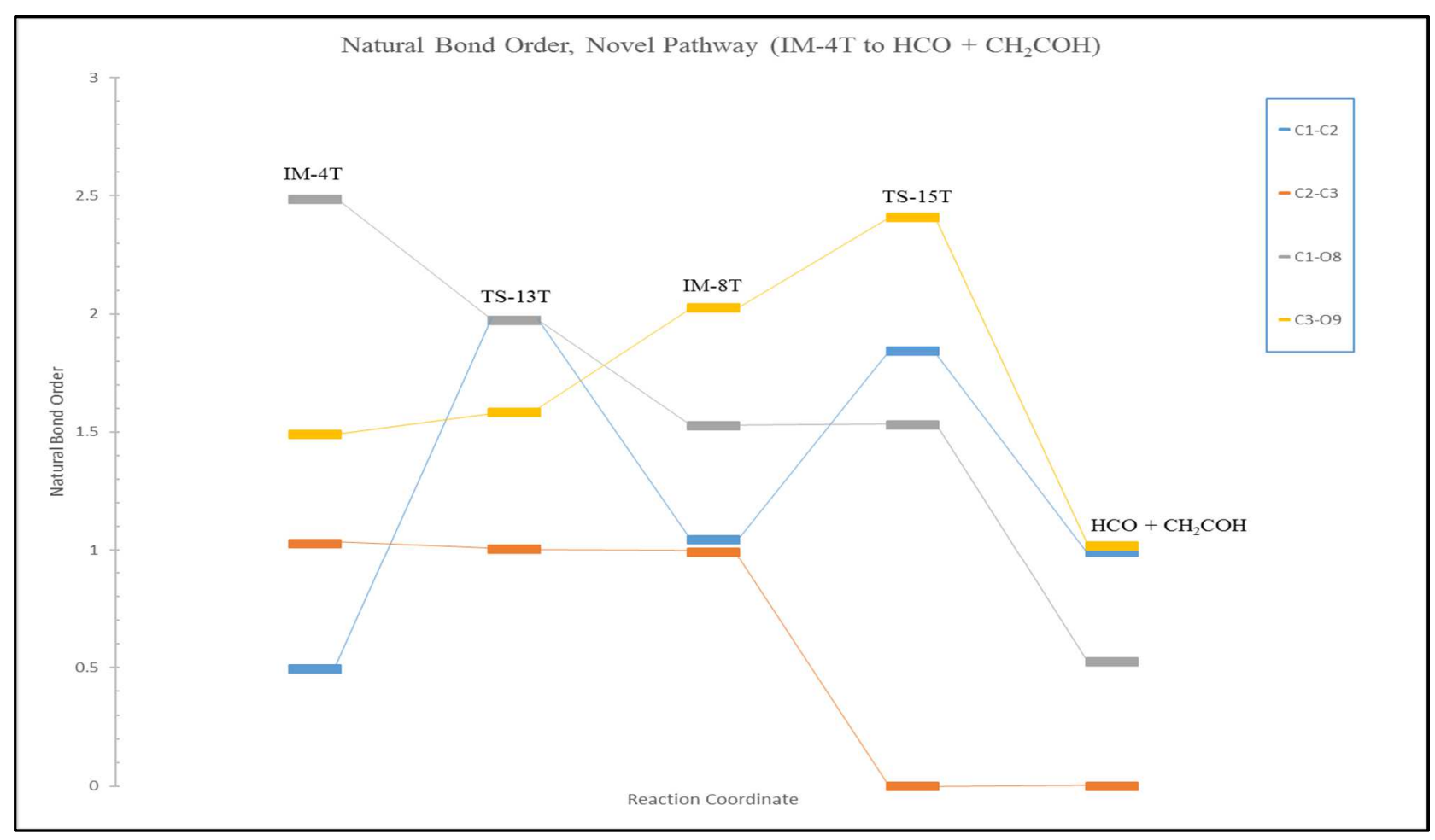

Figure 37. NBO Data for a novel HCO Pathway (IM-4T), calculated at the M06-2x/cc-pVTZ level of theory. 


\section{Computational Results for $\mathbf{O}\left({ }^{3} \mathbf{P}\right)+3-B u t y n-1-o l$}

The other alkyne combustion reaction that has been investigated and presented here is the reaction of $\mathrm{O}\left({ }^{3} \mathrm{P}\right)+3$-butyn-1-ol. The numbering system for $\mathrm{O}\left({ }^{3} \mathrm{P}\right)+3$-butyn-1-ol is shown in Figure 38.

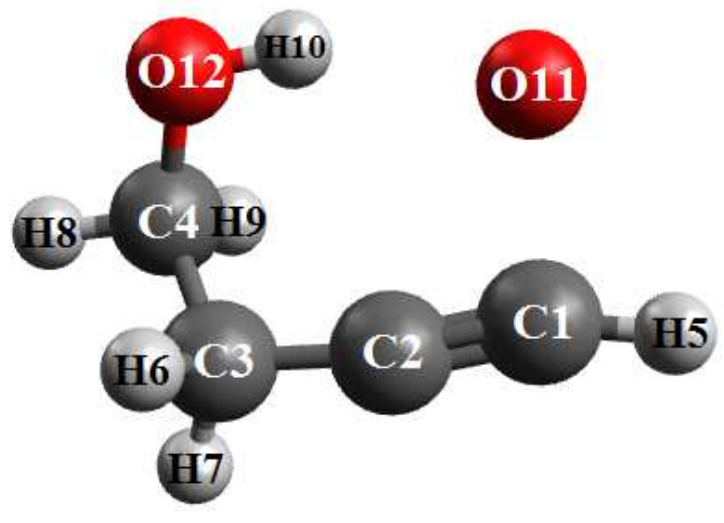

Figure 38. Numbering scheme for the $\mathrm{O}\left({ }^{3} \mathrm{P}\right)+3$-butyn-1-ol reaction.

\section{Geometries: $\mathbf{O}\left({ }^{3} \mathbf{P}\right)+3$-Butyn-1-ol}

Relevant bond lengths and angles for each IM and TS on the $\mathrm{O}\left({ }^{3} \mathrm{P}\right)+3$-butyn-1-ol triplet and singlet potential energy surfaces have been tabulated and are presented in tables 8 and 9 . All geometries reported here are at the M06-2x/cc-pVTZ and MP2/6-311++G(d,p) levels of theory. As with the propargyl alcohol reaction discussed earlier, there is good agreement between the M06-2x and MP2 geometries with only a few deviations worth noting. First, the optimized structures for TS-3T show a difference of $20.4^{\circ}$ between the M06-2x and MP2 results for the C2C3-C4 bond angle. TS-7T shows a $10.6^{\circ}$ difference between the M06-2x and MP2 results for the C1-C2-C3 bond angle. Again, the bond lengths for all structures across M06-2x and MP2 were in good agreement with minor differences on the order of 0.01 angstroms. 
Table 8. Selected bond lengths and angles for structures on the triplet potential energy surface of $\mathrm{O}\left({ }^{3} \mathrm{P}\right)+3$-butyn-1-ol, calculated at the M06-2x/cc-pVTZ and MP2/6-311++G(d,p) levels of theory. The difference between the two levels, $\Delta$, is also reported. The numbering scheme is shown in Figure 38.

\begin{tabular}{|c|c|c|c|c|c|c|c|c|c|c|}
\hline Species & Level/Basis Set & $\begin{array}{c}\text { C1-C2 } \\
(\AA)\end{array}$ & $\begin{array}{c}\mathrm{C} 2-\mathrm{C} 3 \\
(\AA)\end{array}$ & $\begin{array}{c}\text { C3-C4 } \\
(\AA)\end{array}$ & $\begin{array}{c}\text { C1-O11 } \\
(\AA)\end{array}$ & $\begin{array}{c}\text { C4-O12 } \\
(\AA)\end{array}$ & $\begin{array}{c}<\mathrm{C} 1-\mathrm{C} 2-\mathrm{C} 3 \\
\left({ }^{\circ}\right)\end{array}$ & $\begin{array}{c}<\mathrm{C} 2-\mathrm{C} 3-\mathrm{C} 4 \\
\left({ }^{\circ}\right)\end{array}$ & $\begin{array}{c}<011-\mathrm{C} 1-\mathrm{C} 2 \\
\left({ }^{\circ}\right)\end{array}$ & $\begin{array}{c}<\mathrm{C} 3-\mathrm{C} 4-\mathrm{O} 12 \\
\left({ }^{\circ}\right)\end{array}$ \\
\hline \multirow{3}{*}{ 3-Butyn-1-ol } & M06-2x/cc-pVTZ & 1.198 & 1.461 & 1.531 & - & 1.407 & 176.300 & 110.500 & - & 111.900 \\
\hline & MP2/6-311++G(d,p) & 1.220 & 1.463 & 1.532 & - & 1.416 & 176.000 & 110.700 & - & 111.900 \\
\hline & $\Delta$ & 0.022 & 0.002 & 0.001 & - & 0.009 & 0.300 & 0.200 & - & 0.000 \\
\hline \multirow{3}{*}{ TS-1T } & M06-2x/cc-pVTZ & 1.217 & 1.453 & 1.541 & 2.008 & 1.398 & 170.800 & 110.900 & 99.900 & 112.100 \\
\hline & MP2/6-311++G(d,p) & 1.213 & 1.454 & 1.541 & 1.860 & 1.405 & 170.200 & 112.200 & 101.600 & 112.200 \\
\hline & $\Delta$ & 0.004 & 0.001 & 0.000 & 0.148 & 0.007 & 0.600 & 1.300 & 1.700 & 0.100 \\
\hline \multirow{3}{*}{ IM-1T } & M06-2x/cc-pVTZ & 1.413 & 1.462 & 1.542 & 1.234 & 1.398 & 137.700 & 114.100 & 123.500 & 112.300 \\
\hline & MP2/6-311++G(d,p) & 1.452 & 1.474 & 1.543 & 1.205 & 1.405 & 134.800 & 114.600 & 124.000 & 112.800 \\
\hline & $\Delta$ & 0.039 & 0.012 & 0.001 & 0.029 & 0.007 & 2.900 & 0.500 & 0.500 & 0.500 \\
\hline \multirow{3}{*}{ TS-2T } & M06-2x/cc-pVTZ & 1.423 & 1.430 & 1.516 & 1.235 & 1.403 & 134.900 & 119.700 & 121.700 & 111.100 \\
\hline & MP2/6-311++G(d,p) & 1.450 & 1.441 & 1.518 & 1.216 & 1.411 & 132.500 & 119.800 & 123.000 & 111.500 \\
\hline & $\Delta$ & 0.027 & 0.011 & 0.002 & 0.019 & 0.008 & 2.400 & 0.100 & 1.300 & 0.400 \\
\hline \multirow{3}{*}{ IM-2T } & M06-2x/cc-pVTZ & 1.435 & 1.454 & 1.501 & 1.227 & 1.415 & 121.400 & 120.800 & 122.900 & 110.600 \\
\hline & $\mathrm{MP} 2 / 6-311+\mathrm{G}(\mathrm{d}, \mathrm{p})$ & 1.470 & 1.459 & 1.505 & 1.201 & 1.422 & 120.300 & 120.200 & 123.700 & 110.700 \\
\hline & $\Delta$ & 0.035 & 0.005 & 0.004 & 0.026 & 0.007 & 1.100 & 0.600 & 0.800 & 0.100 \\
\hline \multirow{3}{*}{ TS-3T } & M06-2x/cc-pVTZ & 1.447 & 1.448 & 1.485 & 1.227 & 1.407 & 123.300 & 112.200 & 124.800 & 112.200 \\
\hline & MP2/6-311++G(d,p) & 1.456 & 1.456 & 1.493 & 1.226 & 1.413 & 122.100 & 132.600 & 125.100 & 112.600 \\
\hline & $\Delta$ & 0.009 & 0.008 & 0.008 & 0.001 & 0.006 & 1.200 & 20.400 & 0.300 & 0.400 \\
\hline \multirow{3}{*}{ IM-3T } & M06-2x/cc-pVTZ & 1.517 & 1.467 & 1.474 & 1.200 & 1.418 & 114.600 & 130.800 & 124.800 & 111.400 \\
\hline & MP2/6-311++G(d,p) & 1.515 & 1.482 & 1.484 & 1.215 & 1.425 & 116.400 & 133.900 & 125.400 & 112.900 \\
\hline & $\Delta$ & 0.002 & 0.015 & 0.010 & 0.015 & 0.007 & 1.800 & 3.100 & 0.600 & 1.500 \\
\hline \multirow{3}{*}{ TS-4T } & M06-2x/cc-pVTZ & 2.184 & 1.332 & 1.481 & 1.185 & 1.412 & 90.600 & 133.100 & 116.300 & 111.500 \\
\hline & MP2/6-311++G(d,p) & 2.130 & 1.329 & 1.486 & 1.200 & 1.419 & 90.800 & 133.700 & 116.900 & 111.800 \\
\hline & $\Delta$ & 0.054 & 0.003 & 0.005 & 0.015 & 0.007 & 0.200 & 0.600 & 0.600 & 0.300 \\
\hline \multirow{3}{*}{$\begin{array}{c}\mathrm{HCO}+ \\
\mathrm{CH} 2 \mathrm{CCH} 2 \mathrm{OH}\end{array}$} & M06-2x/cc-pVTZ & - & 1.307 & 1.480 & 1.173 & 1.415 & - & 136.600 & - & 112.200 \\
\hline & MP2/6-311++G(d,p) & - & 1.292 & 1.482 & 1.186 & 1.421 & - & 138.700 & - & 112.800 \\
\hline & $\Delta$ & - & 0.015 & 0.002 & 0.013 & 0.006 & - & 2.100 & - & 0.600 \\
\hline \multirow{3}{*}{ TS-5T } & M06-2x/cc-pVTZ & 2.148 & 1.302 & 1.515 & 1.191 & 1.408 & 117.600 & 124.800 & 109.800 & 111.200 \\
\hline & MP2/6-311++G(d,p) & 2.029 & 1.284 & 1.516 & 1.194 & 1.415 & 117.500 & 126.200 & 115.200 & 111.300 \\
\hline & $\Delta$ & 0.119 & 0.018 & 0.001 & 0.003 & 0.007 & 0.100 & 1.400 & 5.400 & 0.100 \\
\hline \multirow{3}{*}{$\begin{array}{c}\mathrm{HCO}+ \\
\mathrm{CHCHCH} 2 \mathrm{OH}\end{array}$} & M06-2x/cc-pVTZ & - & 1.303 & 1.509 & 1.171 & 1.409 & - & 124.800 & - & 111.800 \\
\hline & MP2/6-311++G(d,p) & - & 1.284 & 1.516 & 1.194 & 1.415 & - & 126.200 & - & 111.300 \\
\hline & $\Delta$ & - & 0.019 & 0.007 & 0.023 & 0.006 & - & 1.400 & - & 0.500 \\
\hline \multirow{3}{*}{ TS-6T } & M06-2x/cc-pVTZ & 1.379 & 1.482 & 1.540 & 1.217 & 1.401 & 135.300 & 111.500 & 135.800 & 111.800 \\
\hline & MP2/6-311++G(d,p) & 1.393 & 1.487 & 1.543 & 1.214 & 1.406 & 134.100 & 111.500 & 137.400 & 112.200 \\
\hline & $\Delta$ & 0.014 & 0.005 & 0.003 & 0.003 & 0.005 & 1.200 & 0.000 & 1.600 & 0.400 \\
\hline \multirow{3}{*}{ IM-4T } & M06-2x/cc-pVTZ & 1.436 & 1.491 & 1.523 & 1.194 & 1.408 & 126.600 & 116.900 & 130.300 & 112.200 \\
\hline & MP2/6-311++G(d,p) & 1.456 & 1.497 & 1.526 & 1.198 & 1.416 & 126.600 & 117.300 & 130.100 & 112.500 \\
\hline & $\Delta$ & 0.020 & 0.006 & 0.003 & 0.004 & 0.008 & 0.000 & 0.400 & 0.200 & 0.300 \\
\hline \multirow{3}{*}{ TS-7T } & M06-2x/cc-pVTZ & 2.227 & 1.472 & 1.529 & 1.135 & 1.409 & 124.700 & 114.300 & 114.700 & 112.500 \\
\hline & MP2/6-311++G(d,p) & 2.143 & 1.481 & 1.534 & 1.149 & 1.418 & 114.100 & 113.200 & 113.400 & 112.300 \\
\hline & $\Delta$ & 0.084 & 0.009 & 0.005 & 0.014 & 0.009 & 10.600 & 1.100 & 1.300 & 0.200 \\
\hline \multirow{3}{*}{$\begin{array}{c}\mathrm{CO}+ \\
\mathrm{CHCH} 2 \mathrm{CH} 2 \mathrm{OH}\end{array}$} & M06-2x/cc-pVTZ & - & 1.468 & 1.530 & 1.123 & 1.409 & - & 111.600 & - & 112.200 \\
\hline & MP2/6-311++G(d,p) & - & 1.480 & 1.530 & 1.140 & 1.418 & - & 112.600 & - & 112.300 \\
\hline & $\Delta$ & - & 0.012 & 0.000 & 0.017 & 0.009 & - & 1.000 & - & 0.100 \\
\hline \multirow{3}{*}{ TS-8T } & M06-2x/cc-pVTZ & 1.438 & 1.481 & 1.499 & 1.196 & 1.413 & 123.900 & 121.700 & 130.300 & 112.000 \\
\hline & MP2/6-311++G(d,p) & 1.449 & 1.484 & 1.501 & 1.205 & 1.423 & 124.000 & 122.100 & 129.900 & 112.200 \\
\hline & $\Delta$ & 0.011 & 0.003 & 0.002 & 0.009 & 0.010 & 0.100 & 0.400 & 0.400 & 0.200 \\
\hline
\end{tabular}


Table 9. Continued selected bond lengths and angles for structures on the triplet potential energy surface of $\mathrm{O}\left({ }^{3} \mathrm{P}\right)+3$-butyn-1-ol, calculated at the M06-2x/cc-pVTZ and MP2/6$311++\mathrm{G}(\mathrm{d}, \mathrm{p})$ levels of theory. The difference between the two levels, $\Delta$, is also reported. The numbering scheme is shown in Figure 38.

\begin{tabular}{|c|c|c|c|c|c|c|c|c|c|c|}
\hline Species & Level/Basis Set & $\begin{array}{c}\text { C1-C2 } \\
(\AA)\end{array}$ & $\begin{array}{c}\mathrm{C} 2-\mathrm{C} 3 \\
(\AA)\end{array}$ & $\begin{array}{c}\text { C3-C4 } \\
(\AA)\end{array}$ & $\begin{array}{c}\text { C1-O11 } \\
(\AA)\end{array}$ & $\begin{array}{c}\text { C4-O12 } \\
(\AA)\end{array}$ & $\begin{array}{c}<\mathrm{C} 1-\mathrm{C} 2-\mathrm{C} 3 \\
\left({ }^{\circ}\right)\end{array}$ & $\begin{array}{c}<\mathrm{C} 2-\mathrm{C} 3-\mathrm{C} 4 \\
\left({ }^{\circ}\right)\end{array}$ & $\begin{array}{c}<\text { O11-C1-C2 } \\
\left({ }^{\circ}\right)\end{array}$ & $\begin{array}{c}<\mathrm{C} 3-\mathrm{C} 4-\mathrm{O} 12 \\
\left({ }^{\circ}\right)\end{array}$ \\
\hline \multirow{3}{*}{ IM-5T } & M06-2x/cc-pVTZ & 1.512 & 1.497 & 1.490 & 1.177 & 1.421 & 114.400 & 122.200 & 127.900 & 112.400 \\
\hline & MP2/6-311++G(d,p) & 1.516 & 1.501 & 1.495 & 1.189 & 1.429 & 114.500 & 121.800 & 127.900 & 112.800 \\
\hline & $\Delta$ & 0.004 & 0.004 & 0.005 & 0.012 & 0.008 & 0.100 & 0.400 & 0.000 & 0.400 \\
\hline \multirow{3}{*}{ TS-9T } & M06-2x/cc-pVTZ & 2.142 & 1.458 & 1.492 & 1.137 & 1.412 & 109.200 & 123.400 & 113.500 & 112.400 \\
\hline & MP2/6-311++G(d,p) & 2.100 & 1.469 & 1.496 & 1.152 & 1.422 & 110.600 & 123.300 & 114.700 & 112.200 \\
\hline & $\Delta$ & 0.042 & 0.011 & 0.004 & 0.015 & 0.010 & 1.400 & 0.100 & 1.200 & 0.200 \\
\hline \multirow{3}{*}{$\begin{array}{c}\mathrm{CO}+ \\
\mathrm{CH} 2 \mathrm{CHCH} 2 \mathrm{OH}\end{array}$} & M06-2x/cc-pVTZ & - & 1.451 & 1.493 & 1.122 & 1.413 & - & 121.800 & - & 112.800 \\
\hline & MP2/6-311++G(d,p) & - & 1.460 & 1.498 & 1.139 & 1.422 & - & 121.500 & - & 112.700 \\
\hline & $\Delta$ & - & 0.009 & 0.005 & 0.017 & 0.009 & - & 0.300 & - & 0.100 \\
\hline \multirow{3}{*}{ TS-10T } & M06-2x/cc-pVTZ & 1.522 & 1.501 & 1.477 & 1.175 & 1.368 & 112.300 & 119.400 & 126.300 & 120.200 \\
\hline & MP2/6-311++G(d,p) & 1.524 & 1.504 & 1.482 & 1.187 & 1.376 & 113.300 & 119.100 & 126.800 & 119.800 \\
\hline & $\Delta$ & 0.002 & 0.003 & 0.005 & 0.012 & 0.008 & 1.000 & 0.300 & 0.500 & 0.400 \\
\hline \multirow{3}{*}{ IM-6T } & M06-2x/cc-pVTZ & 1.511 & 1.536 & 1.494 & 1.178 & 1.356 & 114.400 & 114.900 & 128.800 & 120.600 \\
\hline & MP2/6-311++G(d,p) & 1.514 & 1.538 & 1.501 & 1.191 & 1.364 & 115.000 & 115.100 & 128.800 & 120.200 \\
\hline & $\Delta$ & 0.003 & 0.002 & 0.007 & 0.013 & 0.008 & 0.600 & 0.200 & 0.000 & 0.400 \\
\hline \multirow{3}{*}{ TS-11T } & M06-2x/cc-pVTZ & 2.173 & 1.503 & 1.490 & 1.141 & 1.360 & 107.500 & 115.100 & 112.700 & 120.700 \\
\hline & MP2/6-311++G(d,p) & 2.128 & 1.508 & 1.497 & 1.155 & 1.367 & 109.900 & 115.500 & 114.600 & 120.400 \\
\hline & $\Delta$ & 0.045 & 0.005 & 0.007 & 0.014 & 0.007 & 2.400 & 0.400 & 1.900 & 0.300 \\
\hline \multirow{3}{*}{$\begin{array}{c}\mathrm{CO}+ \\
\mathrm{CH} 2 \mathrm{CH} 2 \mathrm{CHOH}\end{array}$} & M06-2x/cc-pVTZ & \multicolumn{9}{|c|}{ M06-2x did not converge. } \\
\hline & MP2/6-311++G(d,p) & - & 1.496 & 1.499 & 1.140 & 1.370 & - & 112.900 & - & 118.000 \\
\hline & $\Delta$ & \multicolumn{9}{|c|}{ M06-2x did not converge. } \\
\hline \multirow{3}{*}{ TS-12T } & M06-2x/cc-pVTZ & 1.431 & 1.449 & 1.496 & 1.226 & 1.364 & 121.000 & 118.500 & 123.100 & 120.900 \\
\hline & MP2/6-311++G(d,p) & 1.470 & 1.451 & 1.499 & 1.197 & 1.373 & 119.400 & 118.200 & 124.100 & 120.100 \\
\hline & $\Delta$ & 0.039 & 0.002 & 0.003 & 0.029 & 0.009 & 1.600 & 0.300 & 1.000 & 0.800 \\
\hline \multirow{3}{*}{ IM-7T } & M06-2x/cc-pVTZ & 1.425 & 1.491 & 1.507 & 1.231 & 1.350 & 121.900 & 114.400 & 123.100 & 119.100 \\
\hline & MP2/6-311++G(d,p) & 1.456 & 1.493 & 1.514 & 1.207 & 1.355 & 121.000 & 114.700 & 123.800 & 119.000 \\
\hline & $\Delta$ & 0.031 & 0.002 & 0.007 & 0.024 & 0.005 & 0.900 & 0.300 & 0.700 & 0.100 \\
\hline \multirow{3}{*}{ IM-8T } & M06-2x/cc-pVTZ & 1.513 & 1.490 & 1.460 & 1.203 & 1.362 & 113.800 & 120.900 & 124.800 & 119.300 \\
\hline & MP2/6-311++G(d,p) & 1.516 & 1.495 & 1.468 & 1.217 & 1.370 & 113.800 & 121.000 & 124.900 & 118.500 \\
\hline & \begin{tabular}{|c|}
$\Delta$ \\
\end{tabular} & 0.003 & 0.005 & 0.008 & 0.014 & 0.008 & 0.000 & 0.100 & 0.100 & 0.800 \\
\hline \multirow[b]{2}{*}{ TS-14T } & M06-2x/cc-pVTZ & 2.238 & 1.354 & 1.470 & 1.175 & 1.365 & 104.100 & 122.300 & 119.300 & 118.500 \\
\hline & $\frac{\mathrm{MP} 2 / 6-311++\mathrm{G}(\mathrm{d}, \mathrm{p})}{4 \Delta}$ & \multicolumn{9}{|c|}{ MP2 did not converge. } \\
\hline \multirow{3}{*}{$\begin{array}{c}\mathrm{HCO}+ \\
\mathrm{CH} 2 \mathrm{CHCHOH}\end{array}$} & M06-2x/cc-pVTZ & - & 1.375 & 1.382 & 1.171 & 1.343 & - & 126.300 & - & 125.400 \\
\hline & MP2/6-311++G(d,p) & - & 1.381 & 1.377 & 1.184 & 1.348 & - & 126.400 & - & 126.000 \\
\hline & $\Delta$ & - & 0.006 & 0.005 & 0.013 & 0.005 & - & 0.100 & - & 0.600 \\
\hline \multirow[b]{2}{*}{ TS-15T } & M06-2x/cc-pVTZ & 1.552 & 1.476 & 1.486 & 1.274 & 1.396 & 119.500 & 120.300 & 116.300 & 111.700 \\
\hline & MP2/6-311++G(d,p) & \multicolumn{9}{|c|}{ MP2 did not converge. } \\
\hline \multirow{3}{*}{ IM-9T } & $\begin{array}{c}\Delta \\
\text { M06-2x/cc-pVTZ }\end{array}$ & 1.560 & 1.478 & 1.483 & 1.289 & 1.384 & 119.100 & 122.300 & 113.100 & 114.800 \\
\hline & MP2/6-311++G(d,p) & \multirow{2}{*}{\multicolumn{9}{|c|}{ MP2 did not converge. }} \\
\hline & $\Delta$ & & & & & & & & & \\
\hline \multirow{3}{*}{ TS-16T } & M06-2x/cc-pVTZ & 1.512 & 1.503 & 1.436 & 1.202 & 1.342 & 111.200 & 119.600 & 124.400 & 132.600 \\
\hline & MP2/6-311++G(d,p) & 1.517 & 1.506 & 1.441 & 1.217 & 1.352 & 111.200 & 120.300 & 124.400 & 132.400 \\
\hline & \begin{tabular}{|l|}
$\Delta$ \\
\end{tabular} & 0.005 & 0.003 & 0.005 & 0.015 & 0.010 & 0.000 & 0.700 & 0.000 & 0.200 \\
\hline & M06-2x/cc-pVTZ & 1.499 & 1.532 & 1.487 & 1.205 & 1.330 & 114.600 & 113.600 & 125.200 & 126.000 \\
\hline IM-10T & MP2/6-311++G(d,p) & 1.504 & 1.534 & 1.496 & 1.219 & 1.339 & 114.900 & 114.000 & 125.200 & 125.400 \\
\hline & $\Delta$ & 0.005 & 0.002 & 0.009 & 0.014 & 0.009 & 0.300 & 0.400 & 0.000 & 0.600 \\
\hline
\end{tabular}




\section{Potential Energy Profiles: $O\left({ }^{3} \mathbf{P}\right)+3$-Butyn-1-ol}

All potential energies presented in this section are referenced relative to the reactants of the reaction, 3-butyn-1-ol and $\mathrm{O}\left({ }^{3} \mathrm{P}\right)$. This reaction has not been investigated computationally prior to this project. All of the PEPs presented here are determined at the M06-2x/cc-pVTZ and $\operatorname{CCSD}(\mathrm{T}) / \mathrm{cc}-\mathrm{pVTZ} / / \mathrm{M} 06-2 \mathrm{x} / \mathrm{cc}-\mathrm{pVTZ}$ levels of theory because, as discussed earlier, the MP2/6$311++\mathrm{G}(\mathrm{d}, \mathrm{p})$ level of theory gave potential energies that were consistently further off from the higher level CCSD(T) results than were the M06-2x results. Again, all single-point energies presented here were calculated at the $\operatorname{CCSD}(\mathrm{T}) / \mathrm{cc}-\mathrm{pVTZ}$ level of theory which were carried out on the M06-2x/cc-pVTZ optimized geometries. Figure 39 shows the PEP for two HCO radical formation pathways at the M06-2x/cc-pVTZ level of theory which, again, demonstrates the importance of collecting single-point energies via $\mathrm{CCSD}(\mathrm{T})$ as $\mathrm{TS}-1 \mathrm{~T}$ violates the definition of a transition state by being lower in energy than IM- 0 at the M06-2x/cc-pVTZ level. 


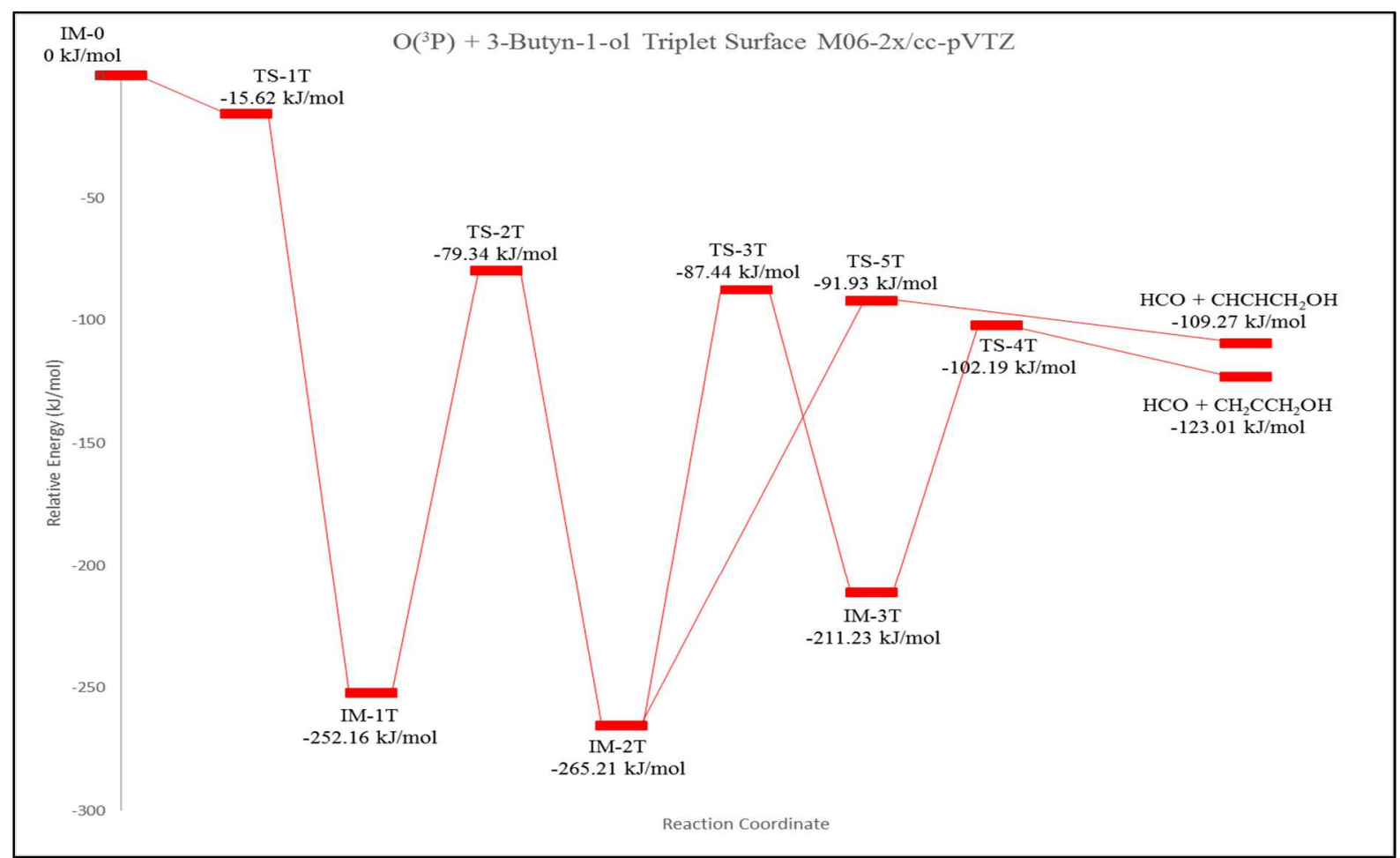

Figure 39. Two HCO formation pathways for $\mathrm{O}\left({ }^{3} \mathrm{P}\right)+3$-butyn-1-ol, M06-2x/cc-pVTZ level.

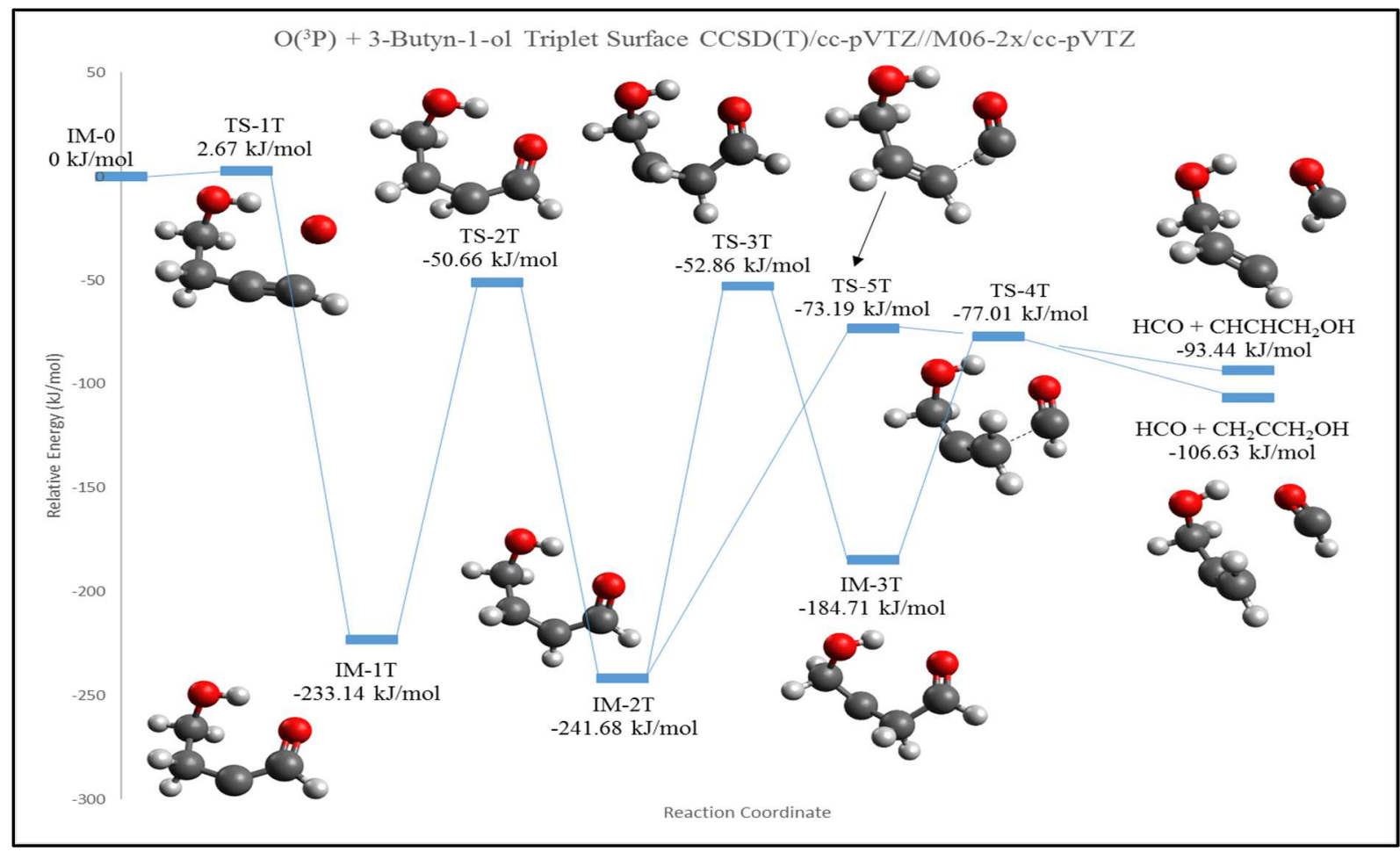

Figure 40. Two HCO formation pathways for $\mathrm{O}\left({ }^{3} \mathrm{P}\right)+3$-butyn-1-ol, $\mathrm{CCSD}(\mathrm{T}) / \mathrm{M} 06-2 \mathrm{x} / \mathrm{cc}-$ pVTZ level. 
As seen in Figure 40, the lowest energy pathway for HCO dissociation involves two Hshifts taking place. After the formation of the adduct (IM-1T), a hydrogen shift takes place from C3 to C2, forming IM-2T. From IM-2T, HCO dissociation occurs via TS-5T at a relative energy of $-73.19 \mathrm{~kJ} / \mathrm{mol}$. However, IM-2T may undergo a second hydrogen shift from C3 to C2 via TS$3 \mathrm{~T}$ which then results in $\mathrm{HCO}$ dissociation at a relative energy of $-106.63 \mathrm{~kJ} / \mathrm{mol}$. Figure 41 shows another PEP for two additional pathways: one HCO radical pathway and one HOC radical pathway. It is worth noting that TS-13T did not converge at the M06-2x/cc-pVTZ level of theory, and so the MP2/6-311++G(d,p) structure and relative energy were used to construct this PEP.

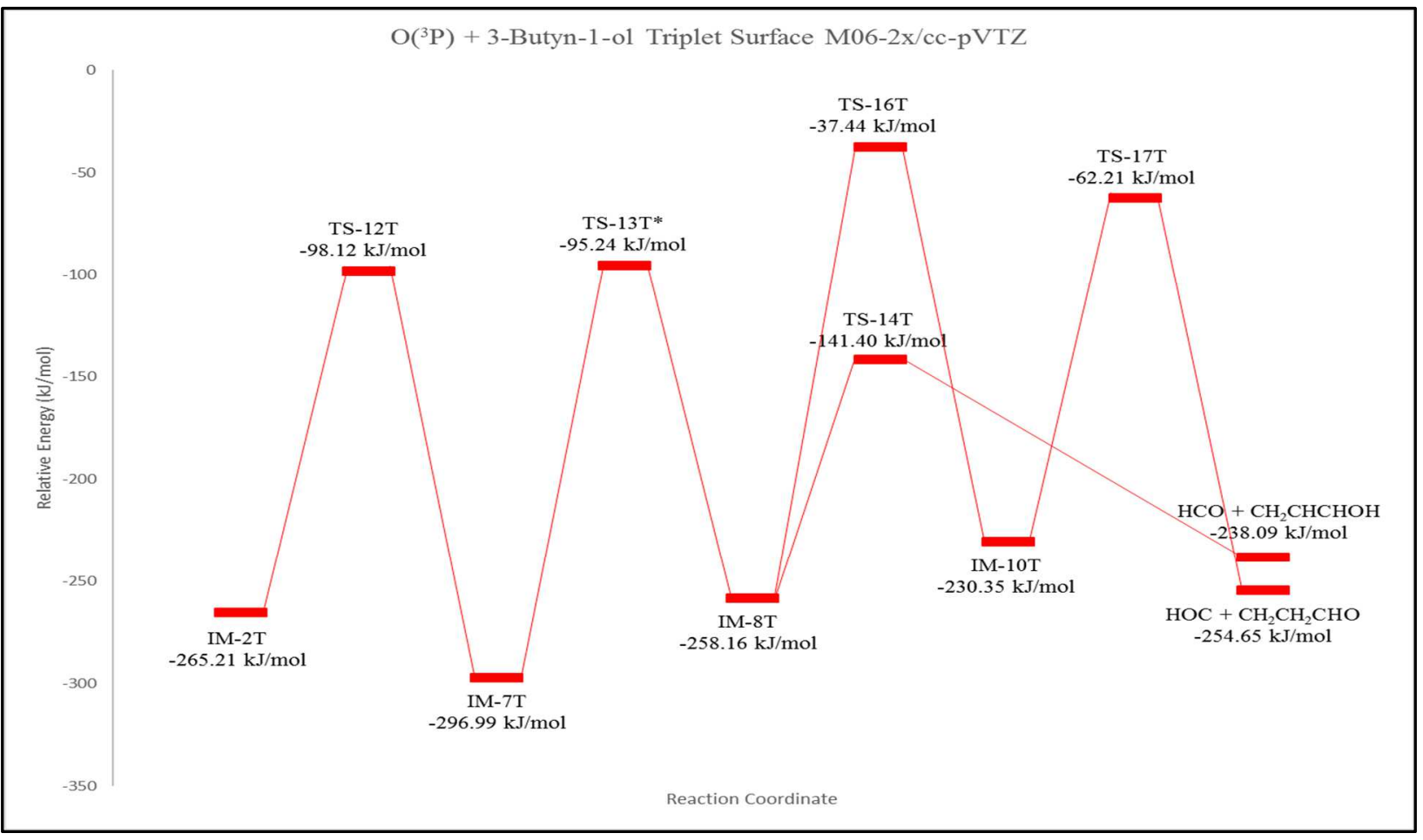

Figure 41. Additional $\mathrm{HCO} / \mathrm{HOC}$ formation pathways for $\mathrm{O}\left({ }^{3} \mathrm{P}\right)+3$-butyn-1-ol, $\mathrm{M} 06-2 \mathrm{x} / \mathrm{cc}-$ pVTZ level. 
Figure 42 shows the CCSD(T)//M06-2x/cc-pVTZ energies of the HCO and HOC radical production pathways shown in Figure 41. Figure 42 reveals the two lowest energy pathways that result in $\mathrm{HCO}$ radical formation, based upon the isomerization of HOC as discussed earlier. Both pathways split off from IM-2T (Figure 40). Similar mechanisms are observed for the $\mathrm{O}\left({ }^{3} \mathrm{P}\right)+3-$ butyn-1-ol reaction as were obtained for the $\mathrm{O}\left({ }^{3} \mathrm{P}\right)+$ propargyl alcohol reaction, namely the role of the hydroxyl substituent engaging in the reaction mechanism. As seen in Figure 41, the hydroxyl group on $\mathrm{C} 4$ eventually forms the HOC product via TS-17T. The final pathway presented here is another HOC dissociation pathway and is shown in Figures 43 and 44.

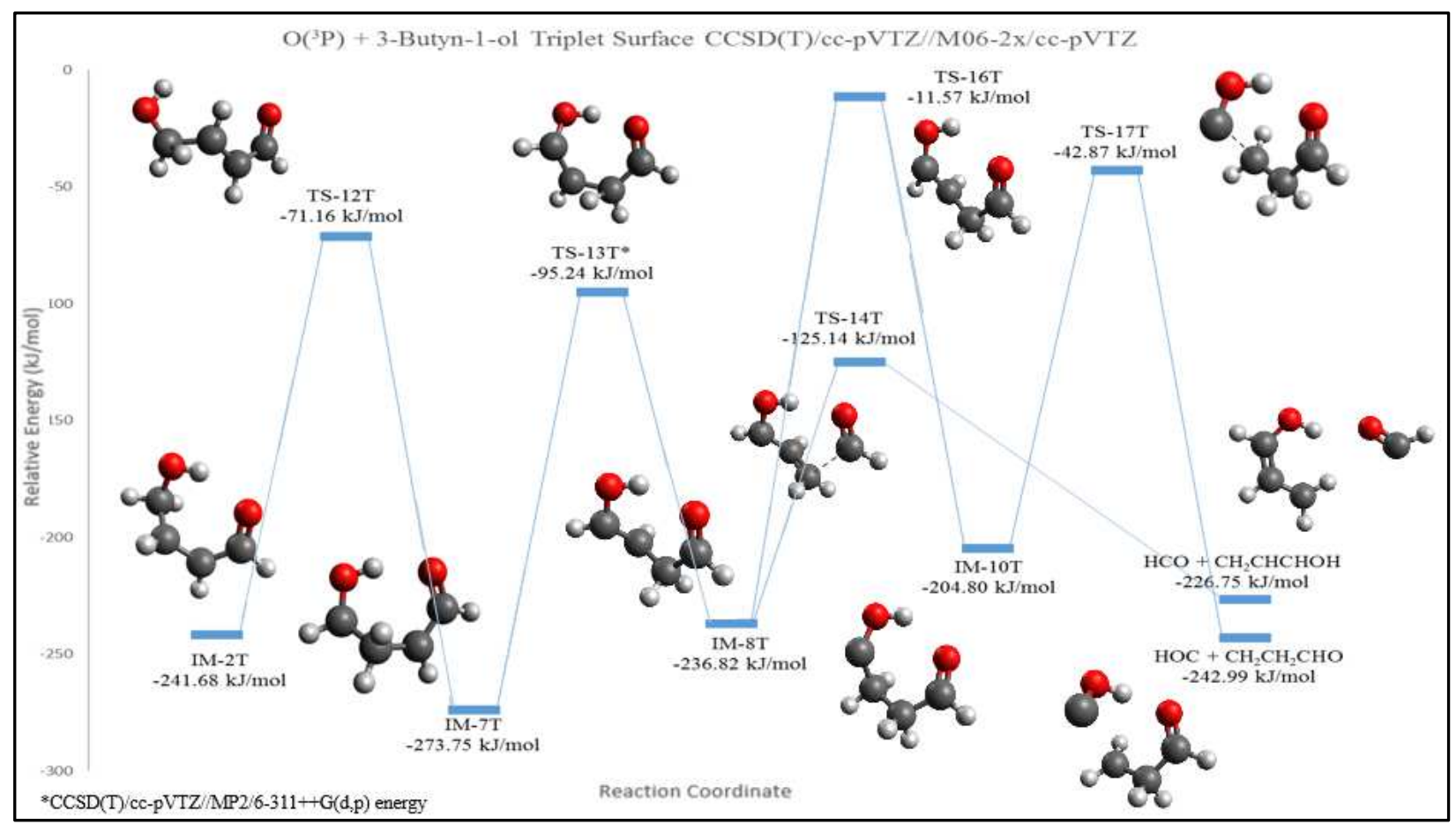

Figure 42. Additional $\mathrm{HCO} / \mathrm{HOC}$ formation pathways for $\mathrm{O}\left({ }^{3} \mathrm{P}\right)+3$-butyn-1-ol, $\mathrm{CCSD}(\mathrm{T}) / / \mathrm{M} 06-2 \mathrm{x} / \mathrm{cc}-\mathrm{pVTZ}$ level. 


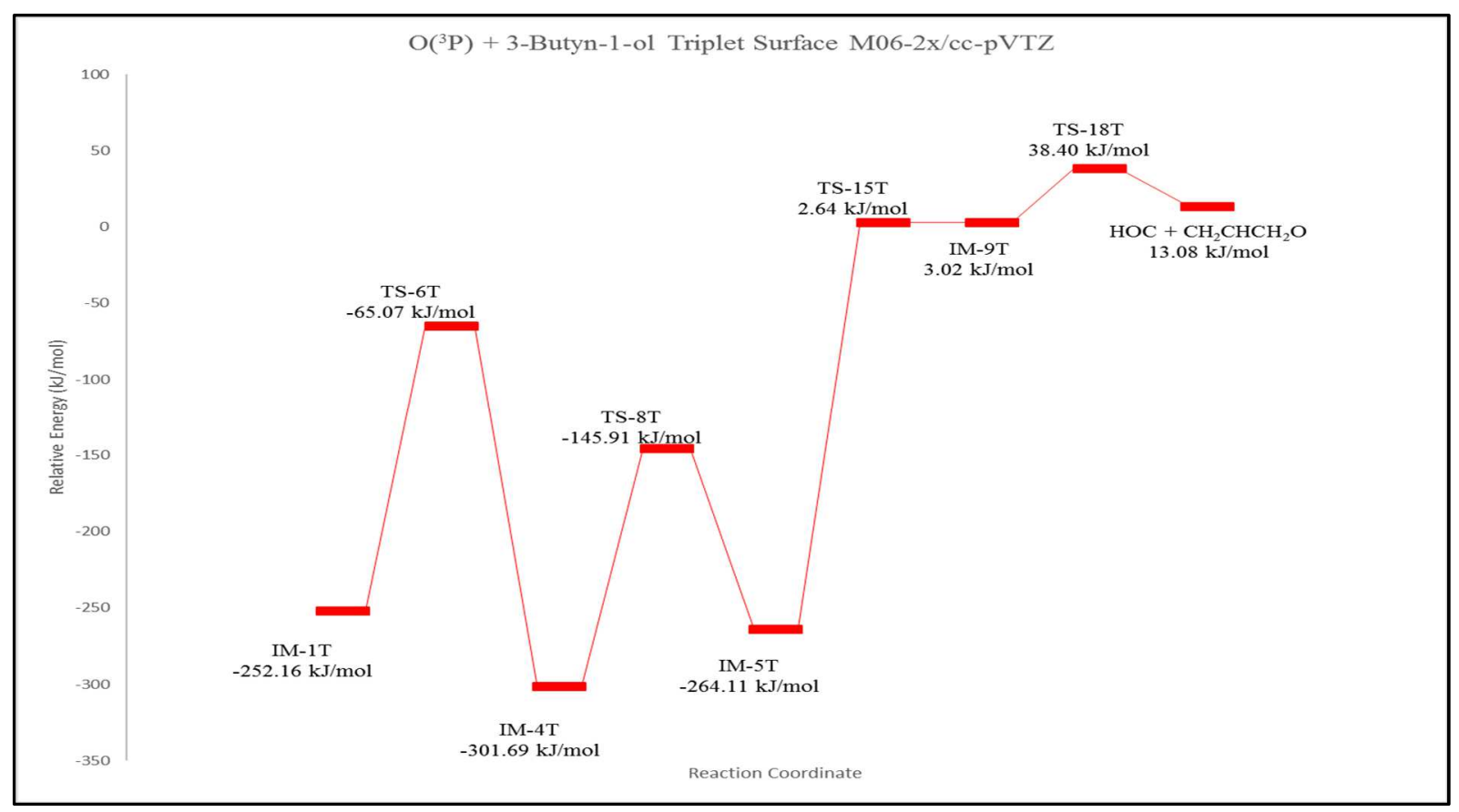

Figure 43. Final HOC dissociation pathway found for $\mathrm{O}\left({ }^{3} \mathrm{P}\right)+3$-butyn-1-ol, M06-2x/cc-pVTZ level. 
Figure 44 reveals that the hydroxyl group plays an important role in the formation of HOC. Similar to a couple of the novel pathways for the propargyl alcohol reaction, a hydrogen atom shifts from $\mathrm{O} 12$ to $\mathrm{O} 11$ via TS-15T, which is followed by the dissociation of HOC via TS$18 \mathrm{~T}$.

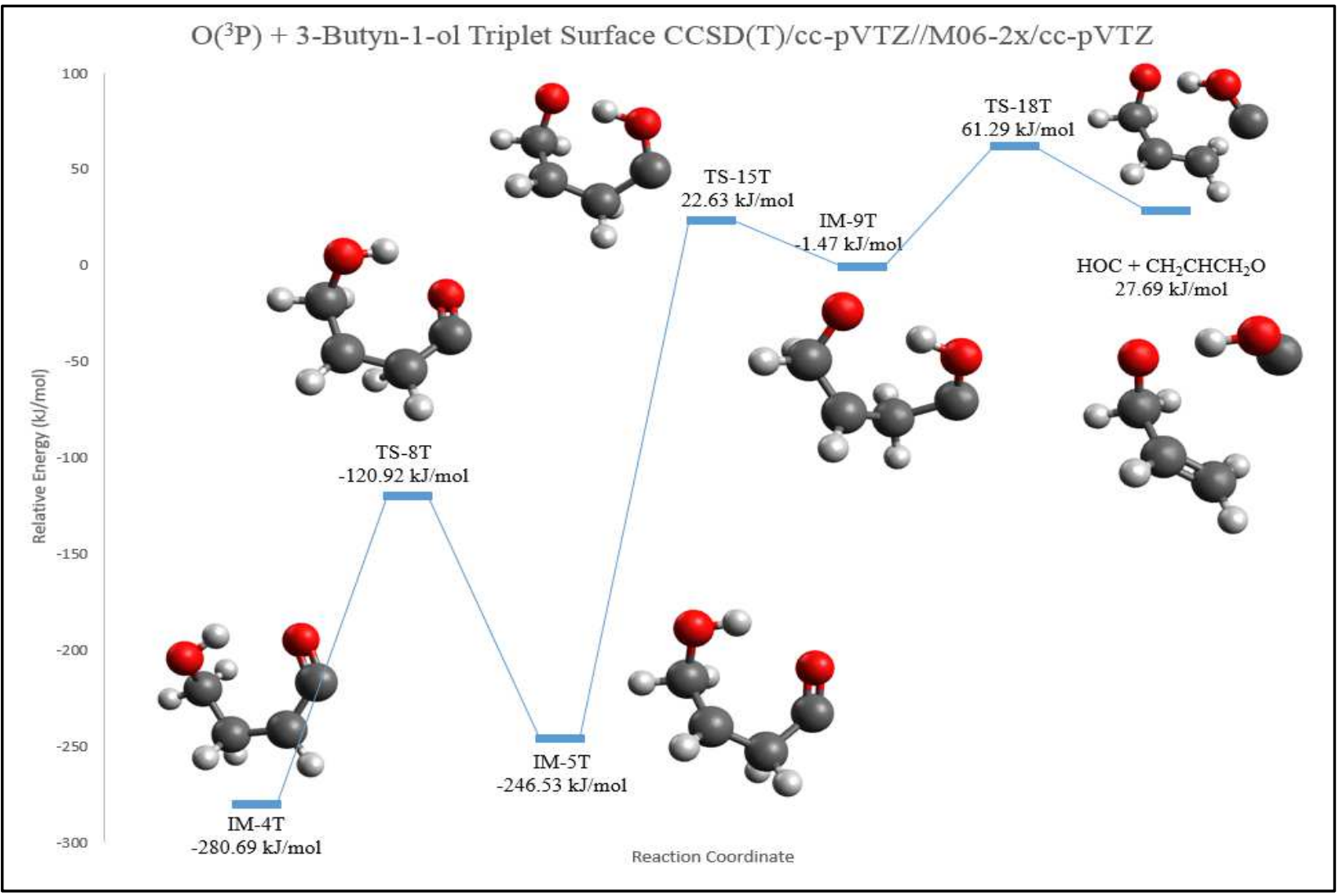

Figure 44. Final HOC dissociation pathway found for $\mathrm{O}\left({ }^{3} \mathrm{P}\right)+3$-butyn-1-ol, $\mathrm{CCSD}(\mathrm{T}) / / \mathrm{M} 06-$ 2x/cc-pVTZ. 
NRT Analysis: $O\left({ }^{3} \mathbf{P}\right)+3-B u t y n-1-o l$

NSD and NBO data for the reaction of $\mathrm{O}\left({ }^{3} \mathrm{P}\right)+3$-butyn-1-ol is shown in Figures 45-54. Again, only the $\mathrm{C}$ and $\mathrm{O}$ atoms are shown on the plots to keep them from being too cluttered. NSD and NBO plots for the first HCO dissociation pathway (Figure 39) are shown in Figures 45 and 46, respectively. Each individual pathway for $\mathrm{HCO} / \mathrm{HOC}$ radical has its own NSD and NBO plots.

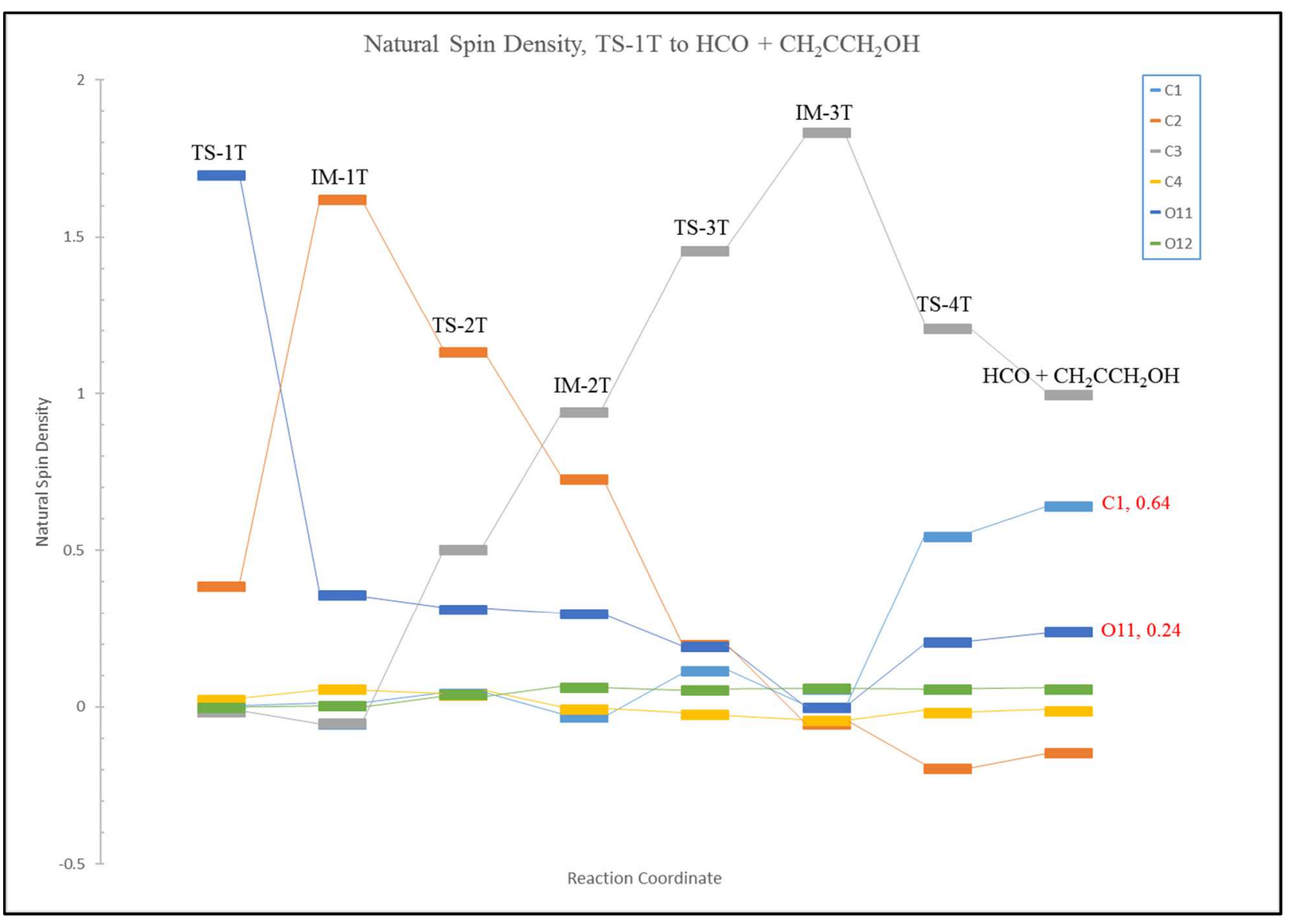

Figure 45. NSD Data for the HCO pathway shown in Figure 40, calculated at the M06-2x/ccpVTZ level of theory. 
As seen in Figure 45, similar trends are observed for the HCO dissociation from 3-butyn1-ol and from propargyl alcohol. The reaction begins with almost all of the NSD on the incoming triplet oxygen atom, $\mathrm{O} 11$, which then quickly transfers to $\mathrm{C} 2$ of the system after the formation of the adduct (IM-1T). After the dissociation step for HCO radical formation takes place, NSD is distributed in a similar 1:3 (O:C) ratio giving the HCO radical a total NSD of 1 (including the NSD of H5, not shown here). Figure 46 confirms the bond orders expected for HCO radical, with a C1-O11 NBO of about 2.5.

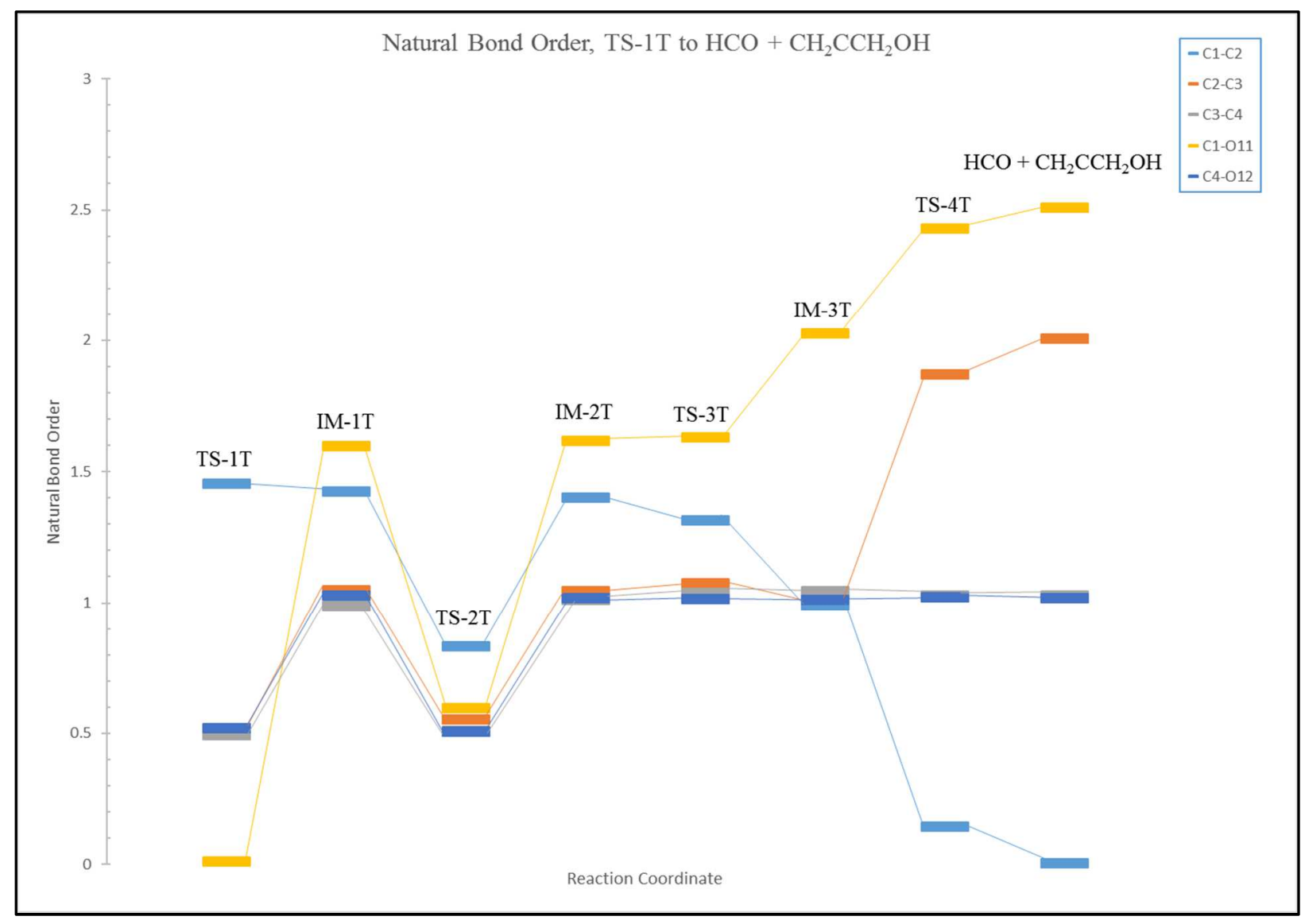

Figure 46. NBO Data for the HCO pathway shown in Figure 40, calculated at the M06-2x/ccpVTZ level of theory. 
The NRT results of all the other $\mathrm{O}\left({ }^{3} \mathrm{P}\right)+3$-butyn-1-ol reaction pathways are presented in figures 47-54. Similar trends are observed in these figures as were observed in the NRT results for the $\mathrm{O}\left({ }^{3} \mathrm{P}\right)+$ propargyl alcohol, namely the 1:3 (O:C) NSD distribution ratio on the HCO radical product and an NSD of $\sim 1$ on the carbon atom of $\mathrm{HOC}$ radical products.

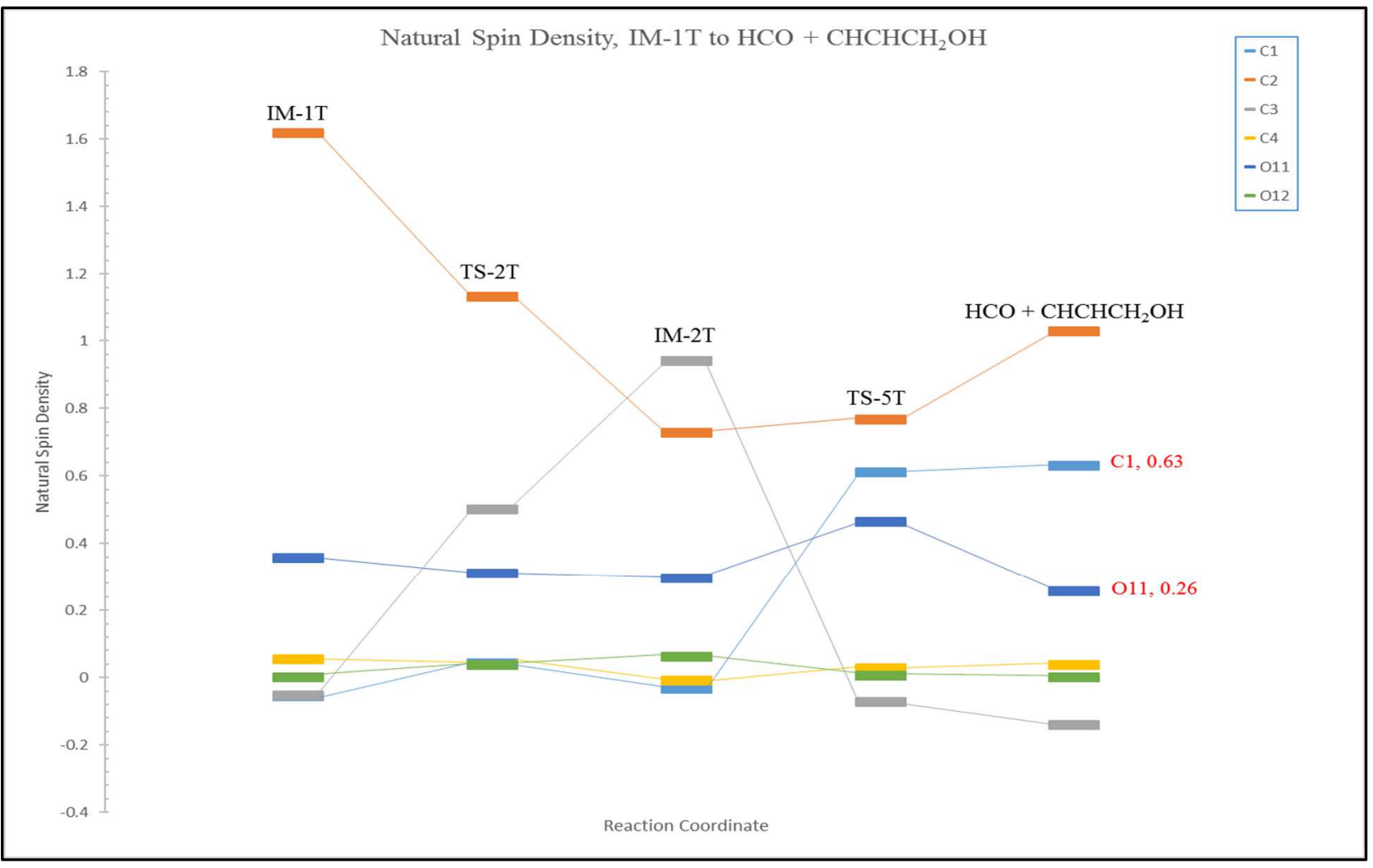

Figure 47. NSD Data for the other HCO pathway shown in Figure 40, calculated at the M062x/cc-pVTZ level of theory. 


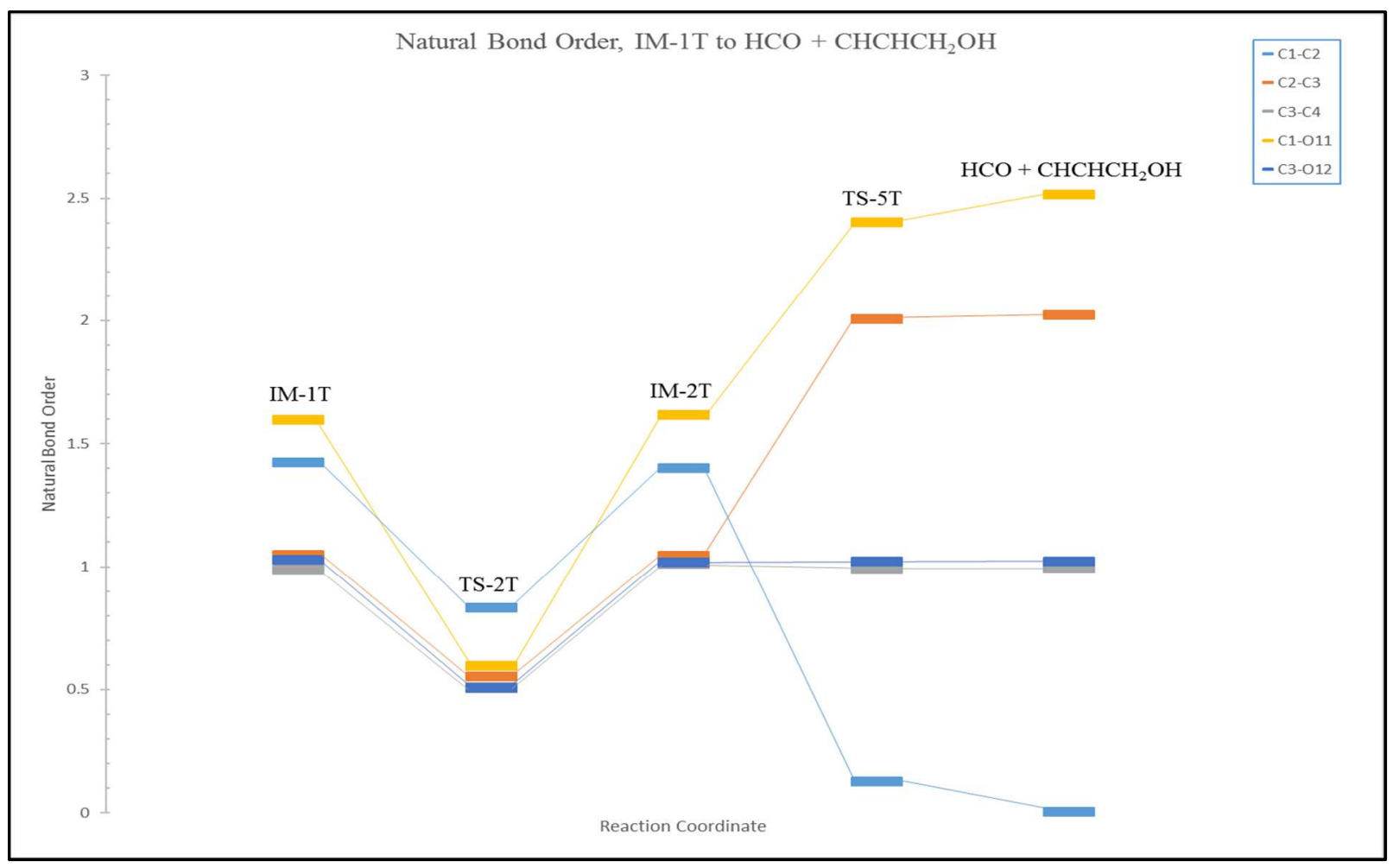

Figure 48. NBO Data for the other HCO pathway shown in Figure 40, calculated at the M06$2 x / c c-p V T Z$ level of theory.

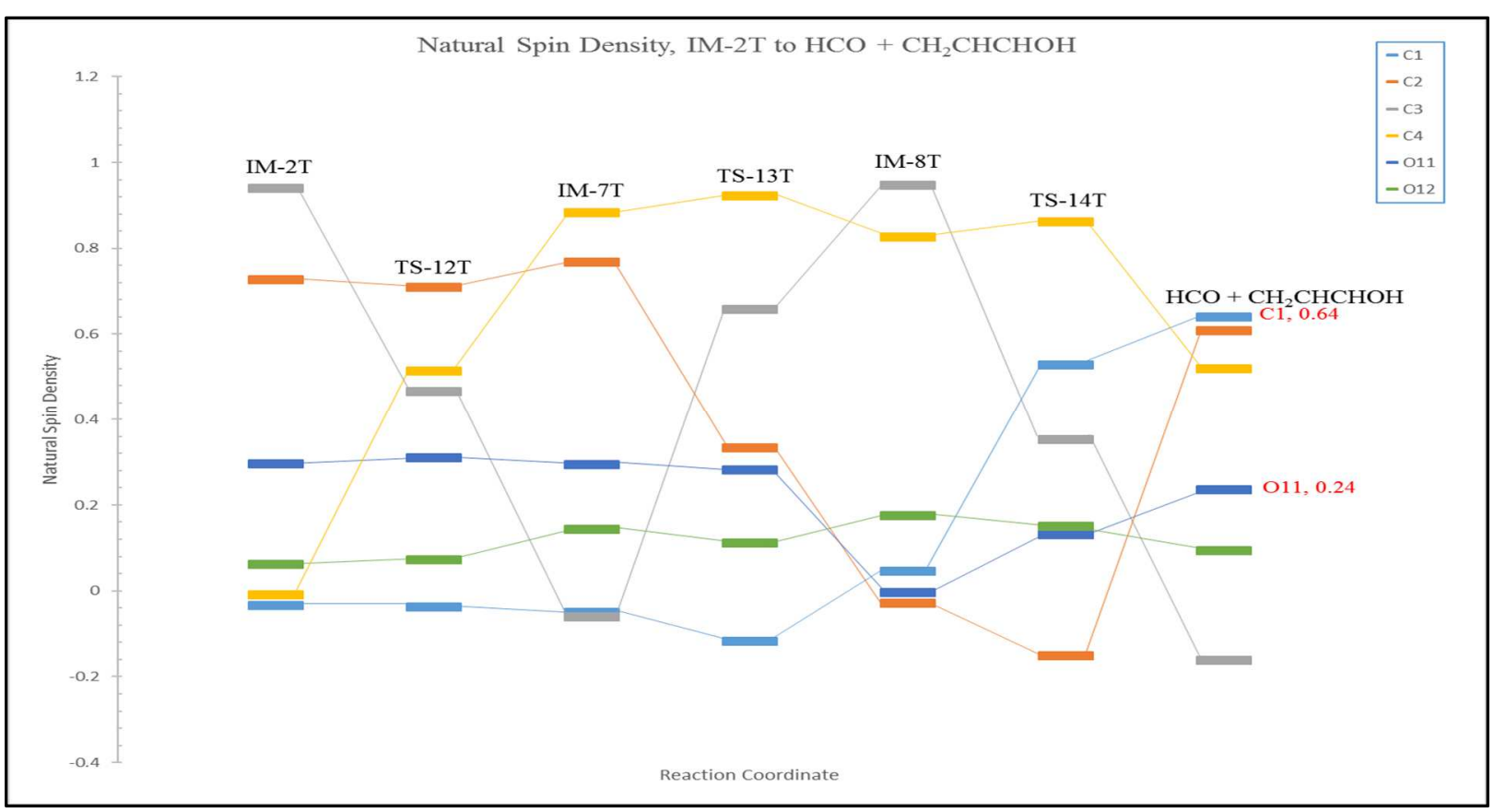

Figure 49. NSD Data for the HCO pathway shown in Figure 42, calculated at the M06-2x/ccpVTZ level of theory. 


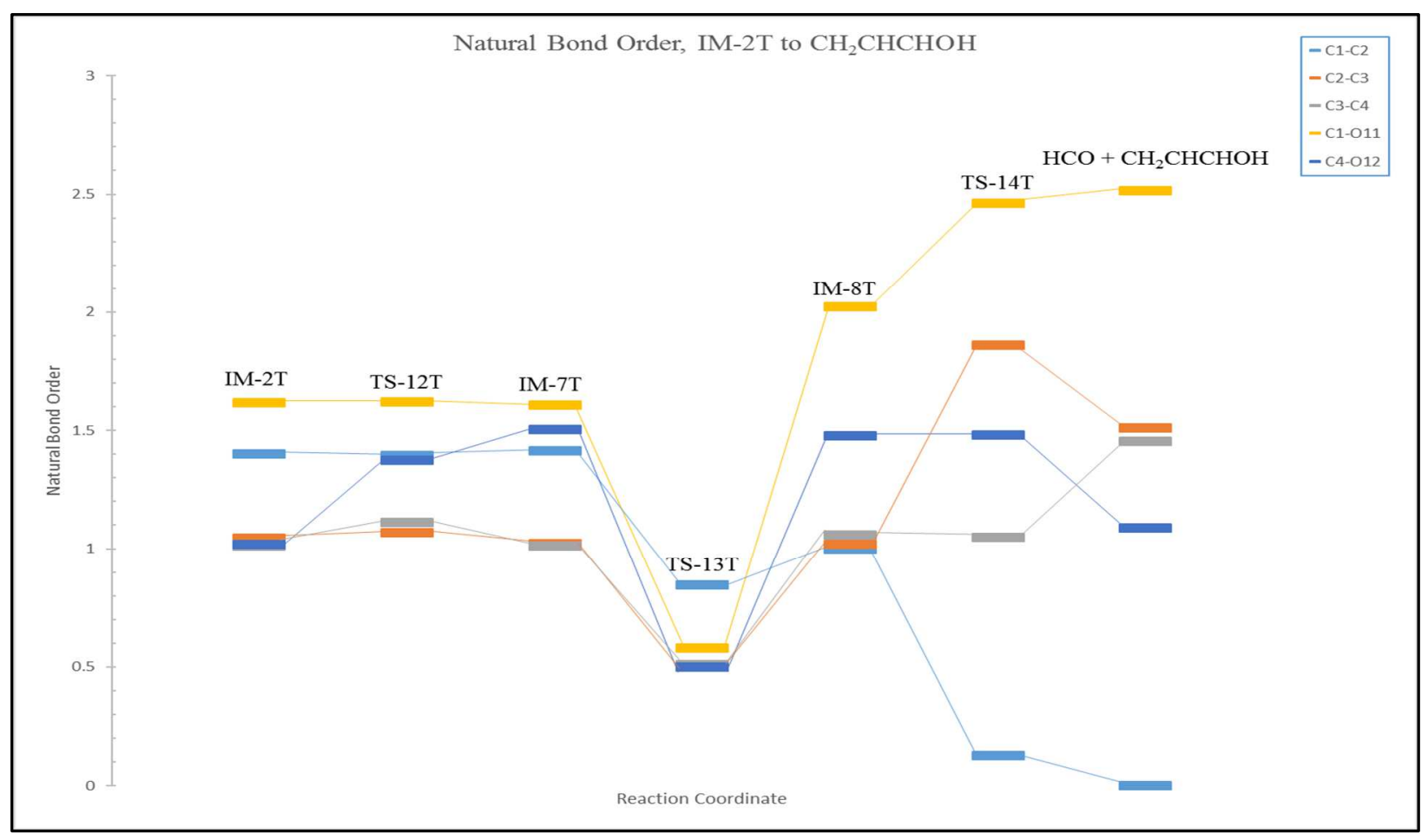

Figure 50. NBO Data for the HCO pathway shown in Figure 42, calculated at the M06-2x/ccpVTZ level of theory.

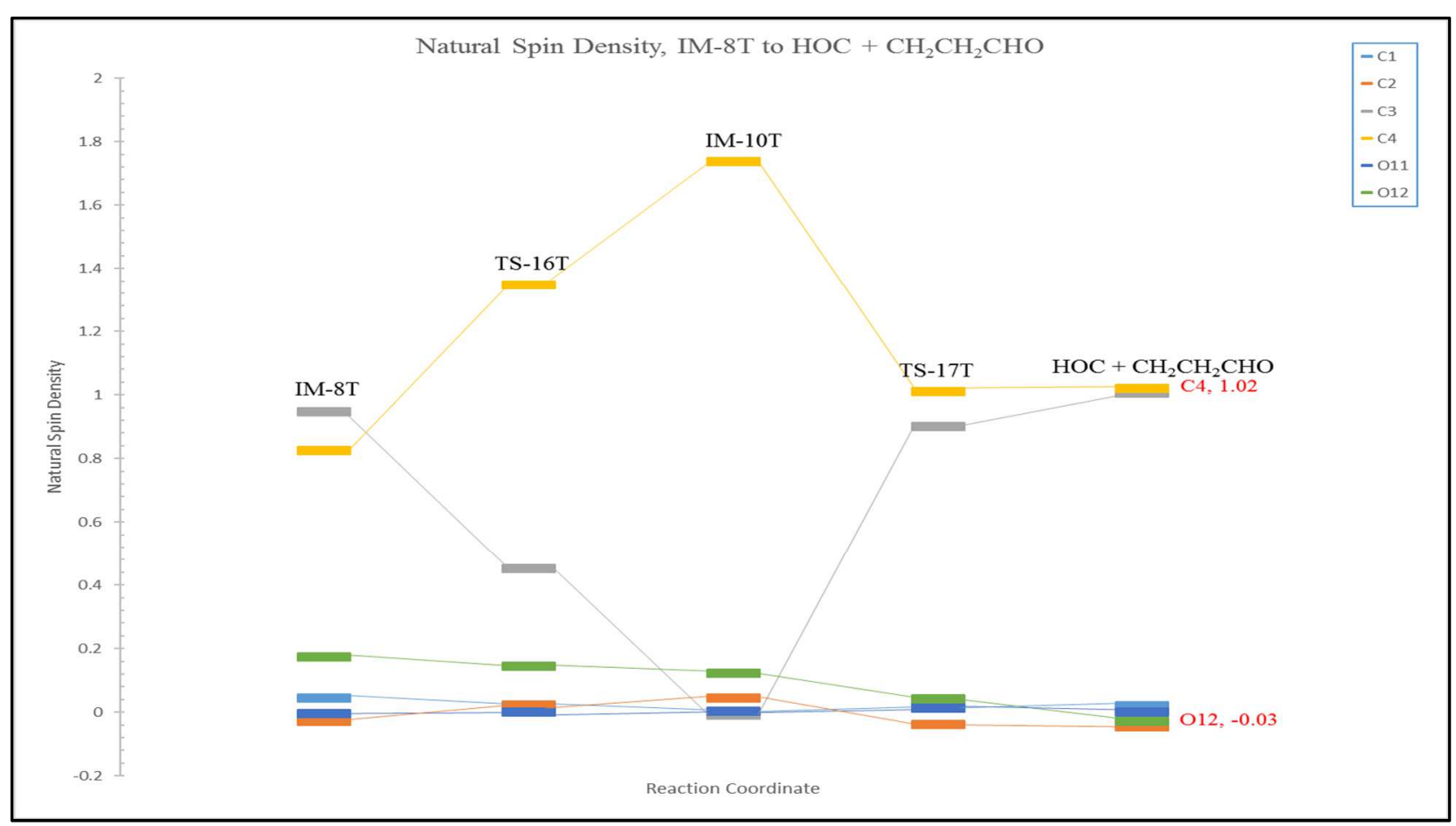

Figure 51. NSD Data for the HOC pathway shown in Figure 42, calculated at the M06-2x/ccpVTZ level of theory. 


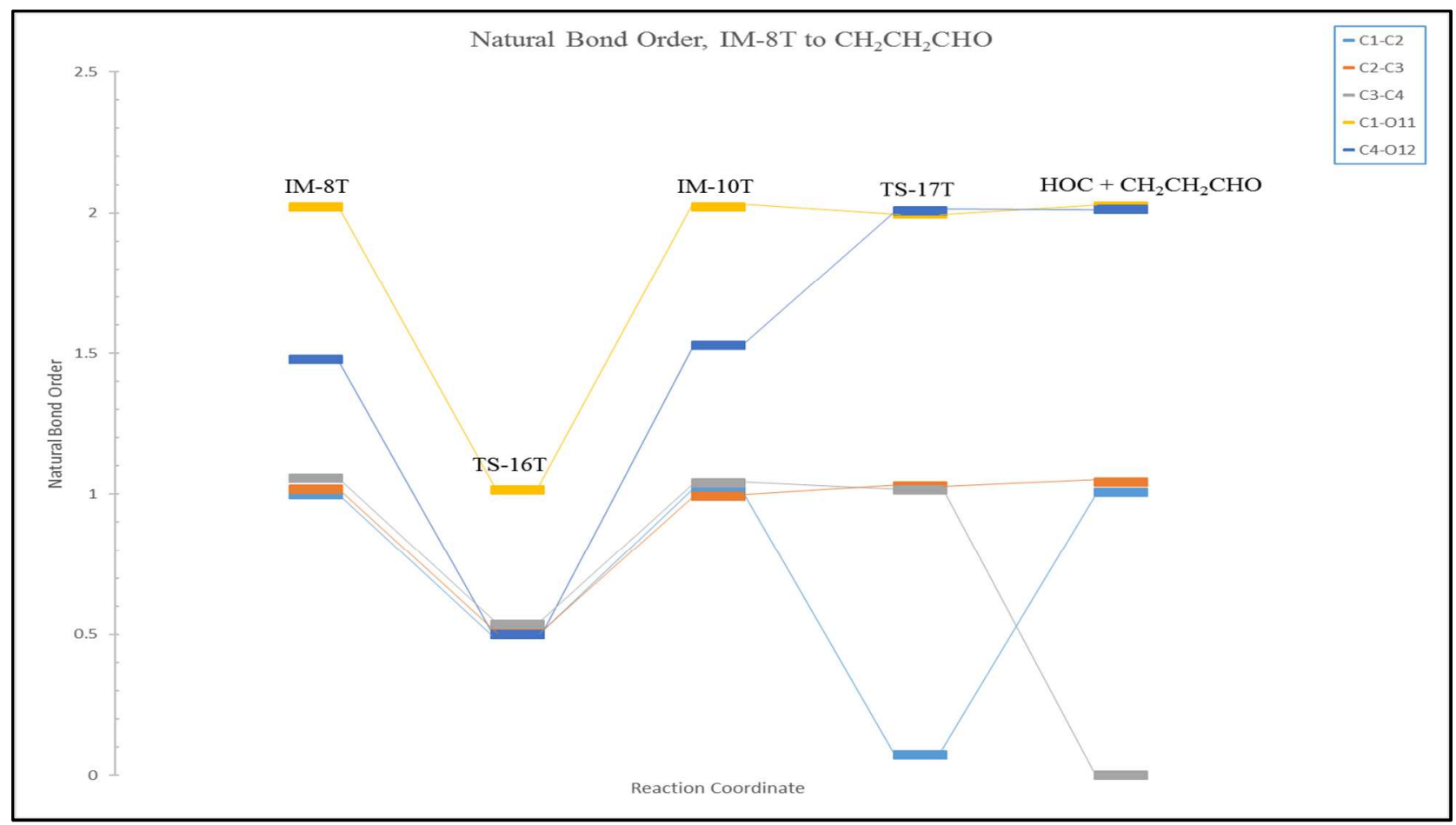

Figure 52. NBO Data for the HOC pathway shown in Figure 42, calculated at the M06-2x/ccpVTZ level of theory.

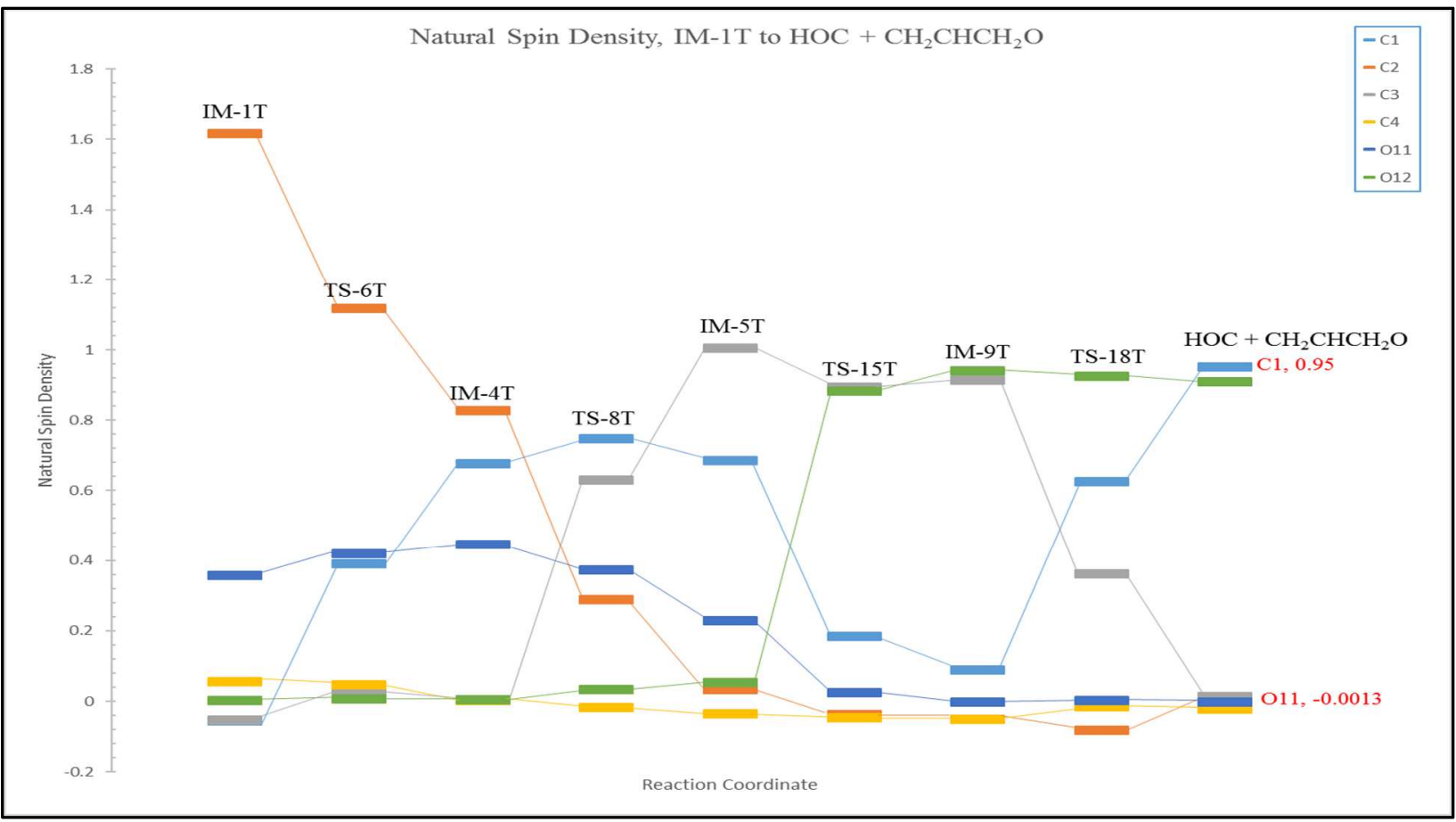

Figure 53. NSD Data for the HOC pathway shown in Figure 44, calculated at the M06-2x/ccpVTZ level of theory. 


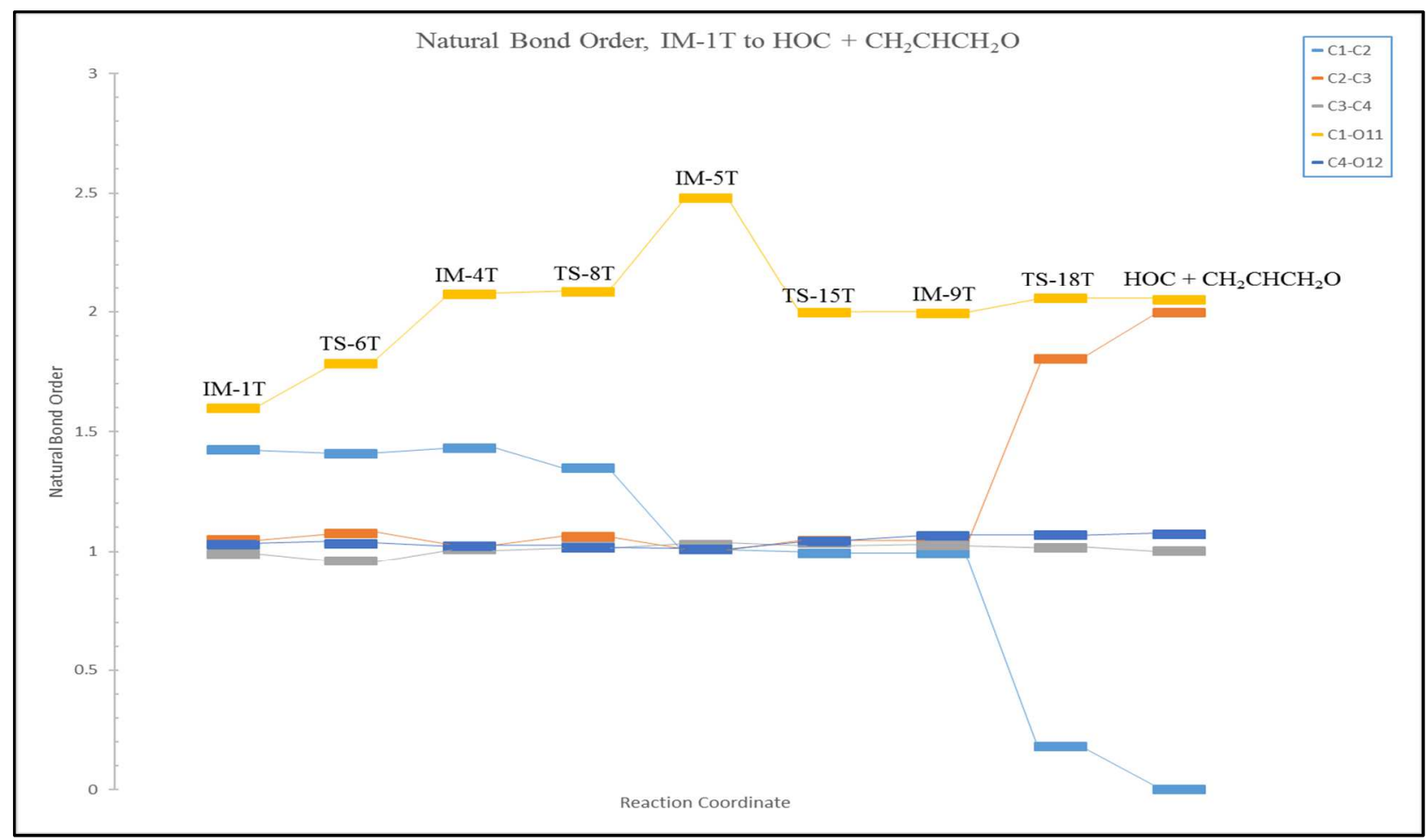

Figure 54. NBO Data for the HOC pathway shown in Figure 44, calculated at the M06-2x/ccpVTZ level of theory.

Following the trends observed in the propargyl alcohol reaction, the HOC formation pathways for $\mathrm{O}\left({ }^{3} \mathrm{P}\right)+3$-butyn-1-ol reaction reveal that nearly all of the NSD is on the $\mathrm{C}$ and $\mathrm{H}$ atoms of the HOC product, with almost none present on the $\mathrm{O}$ atom. The bond orders in $\mathrm{HOC}$ are also consistently lower than they are for $\mathrm{HCO}$, with an $\mathrm{NBO}$ of about 2 between the $\mathrm{C}$ and $\mathrm{O}$ atoms in $\mathrm{HOC}$ and an $\mathrm{NBO}$ of about 2.5 between the $\mathrm{C}$ and $\mathrm{O}$ atoms in $\mathrm{HCO}$. 


\section{Subsequent Pathways}

The final set of results for this project revolves around the formation of additional $\mathrm{HCO} / \mathrm{HOC}$ radical products after one dissociation of $\mathrm{HCO} / \mathrm{HOC}$ radical has already occurred. Of the $\mathrm{O}\left({ }^{3} \mathrm{P}\right)+$ alkyne reactions studied to date, these "subsequent" pathways are totally unique to the reaction of $\mathrm{O}\left({ }^{3} \mathrm{P}\right)+3$-butyn-1-ol and may also aid in explaining the variations of $\mathrm{HCO}$ radical absorption intensity observed in the CRDLAS data. The PEPs shown here were generated by obtaining optimized structures at the M06-2x/cc-pVTZ level and then running single-point energy calculations at the CCSD(T)/cc-pVTZ level. All energies shown here are measured relative to the reactant; i.e., the fragment radical from which $\mathrm{HCO} / \mathrm{HOC}$ radical subsequently dissociates. The first subsequent pathway revealed here branches off from the first PEP shown for $\mathrm{O}\left({ }^{3} \mathrm{P}\right)+3$-butyn-1-ol (Figure 39). After $\mathrm{HCO}$ dissociates, a fragment $\mathrm{CH}_{2} \mathrm{CCH}_{2} \mathrm{OH}$ radical is left over. The reaction pathway for $\mathrm{CH}_{2} \mathrm{CCH}_{2} \mathrm{OH}$ radical to form $\mathrm{HOC}+$ ethylene is shown in Figure 55. The next subsequent pathway branches off from the other fragment product shown in Figure 39. $\mathrm{CHCHCH}_{2} \mathrm{OH}$ radical may also follow a reaction pathway that results in the formation of $\mathrm{HOC}+$ ethylene, which is shown in Figure 56. The final subsequent pathway branches off from the product fragment in Figure 41. The $\mathrm{CH}_{2} \mathrm{CH}_{2} \mathrm{CHO}$ radical may follow a one-step reaction pathway to form $\mathrm{HCO}+$ ethylene, as shown in Figure 57. 


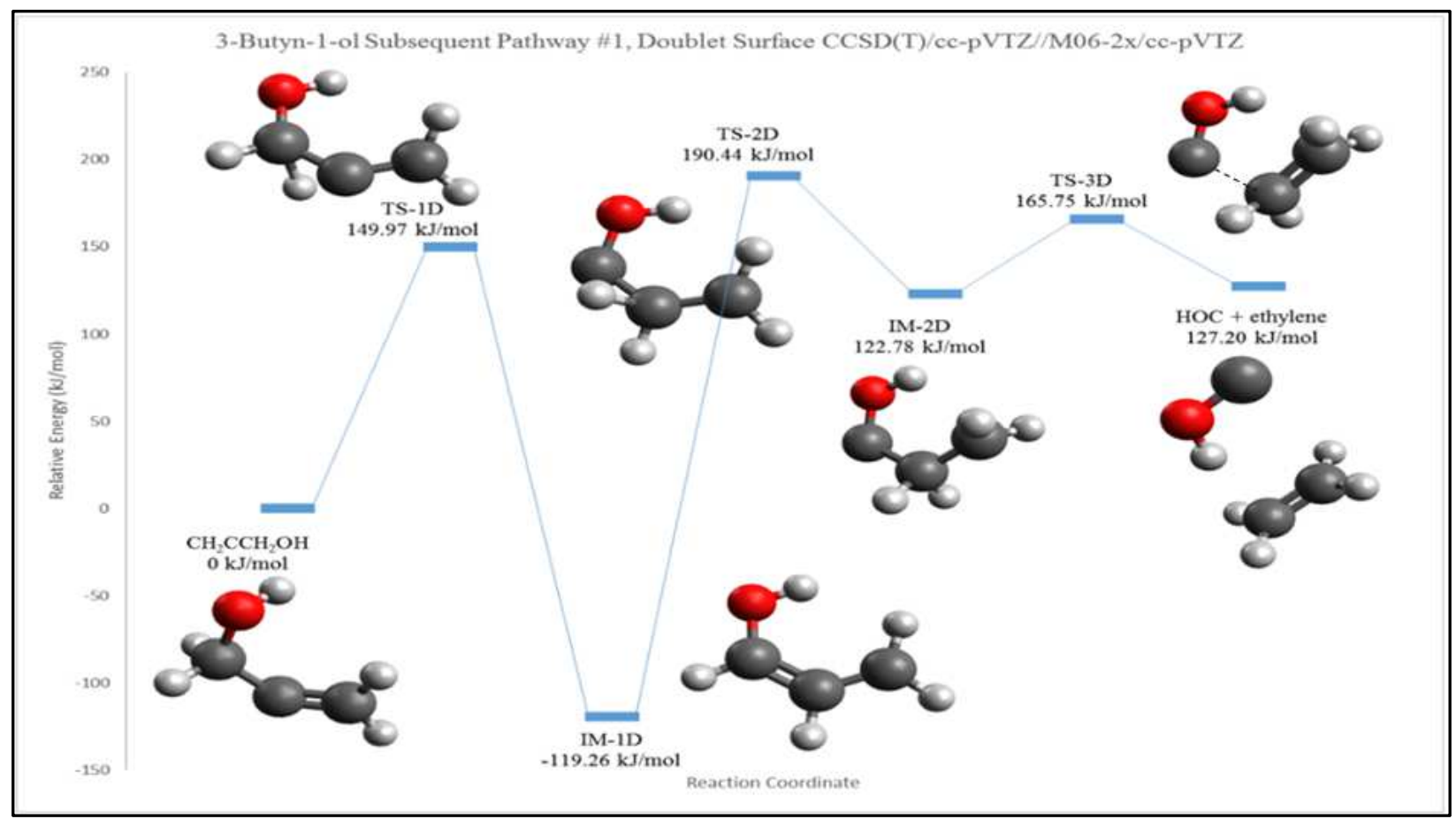

Figure 55. Subsequent dissociation pathway for HOC, CCSD(T)//M06-2x/cc-pVTZ level.

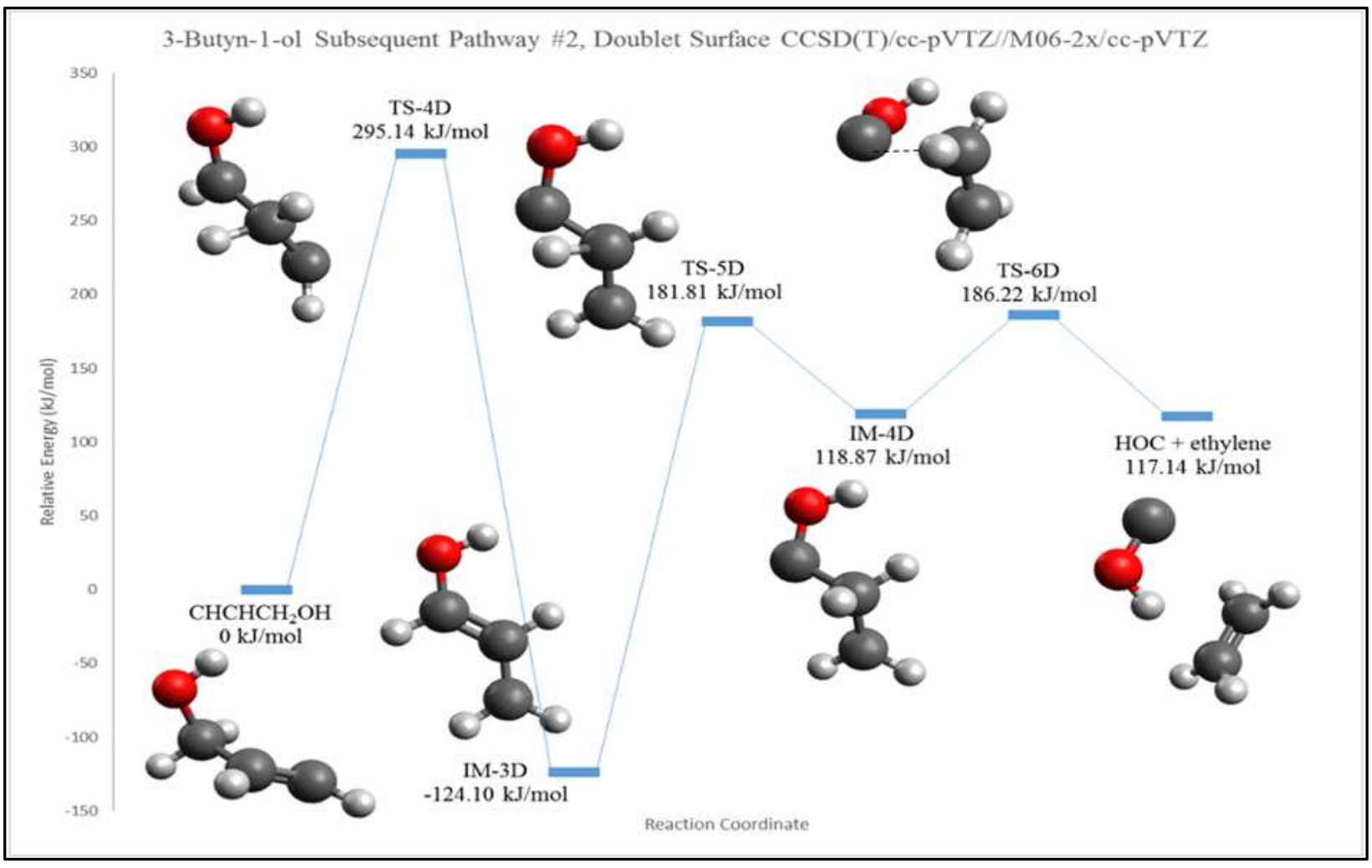

Figure 56. Subsequent dissociation pathway for HOC, CCSD(T)//M06-2x/cc-pVTZ level. 


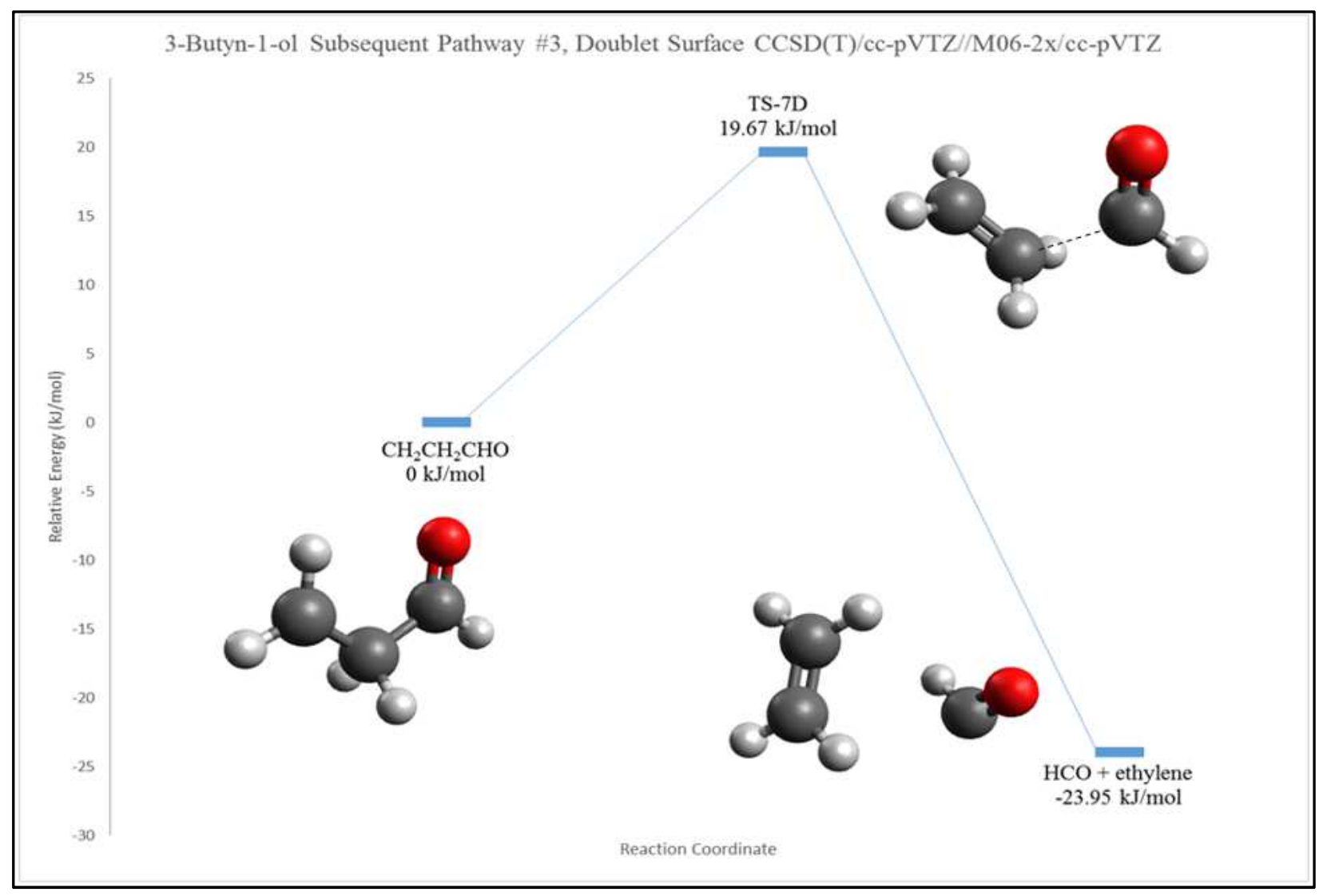

Figure 57. Subsequent dissociation pathway for HCO, CCSD(T)//M06-2x/cc-pVTZ level.

Shown in Figure 57, the subsequent dissociation of $\mathrm{HCO}$ radical from $\mathrm{CH}_{2} \mathrm{CH}_{2} \mathrm{CHO}$ is thermodynamically favorable as the reaction has to overcome a small relative energy barrier of $19.67 \mathrm{~kJ} / \mathrm{mol}$ and results in products that are $23.95 \mathrm{~kJ} / \mathrm{mol}$ lower than the reactant. This subsequent pathway is predicted to be favorable as it has the fewest number of steps, lowest relative energy barrier, and products that are lower in energy than the fragment radical reactant. The subsequent dissociation pathway shown in Figure 57 may very well contribute to the strong HCO radical absorption signal detected for $\mathrm{O}\left({ }^{3} \mathrm{P}\right)+3$-butyn-1-ol during the CRDLAS studies conducted by Buettner. ${ }^{28}$ 


\section{CHAPTER V}

\section{CONCLUSION}

There are many consistencies worth discussing between the reactions of $\mathrm{O}\left({ }^{3} \mathrm{P}\right)+$ propargyl alcohol and $\mathrm{O}\left({ }^{3} \mathrm{P}\right)+3$-butyn-1-ol. With regards to the PEPs presented in this thesis, similar mechanisms are at play in both reactions involving the hydroxyl substituent. The presence of two oxygen atoms in these systems allows for more avenues of formyl radical production, as illustrated in the PEPs. The hydroxyl group has proven to take part in reaction mechanisms that lead to $\mathrm{HCO}$ and $\mathrm{HOC}$ radical formation for both the incoming $\mathrm{O}\left({ }^{3} \mathrm{P}\right)$ atom (O8 for propargyl alcohol and $\mathrm{O} 11$ for 3-butyn-1-ol) and for the hydroxyl oxygen itself (O9 for propargyl alcohol and $\mathrm{O} 12$ for 3-butyn-1-ol). Both formyl radical and isoformyl radical formation may occur via bond breakage at either end of the molecule because of the involvement of the hydroxyl group in these mechanisms.

In addition to the unique $\mathrm{HCO} / \mathrm{HOC}$ radical formation pathways for hydroxyl-substituted alkynes, subsequent $\mathrm{HCO} / \mathrm{HOC}$ dissociations have been demonstrated for the reaction of $\mathrm{O}\left({ }^{3} \mathrm{P}\right)+$ 3-butyn-1-ol. These subsequent dissociations have also been investigated for $\mathrm{O}\left({ }^{3} \mathrm{P}\right)+$ propargyl alcohol, but were never found. The first steps of a possible subsequent $\mathrm{HCO} / \mathrm{HOC}$ dissociation pathway for $\mathrm{O}\left({ }^{3} \mathrm{P}\right)+$ propargyl alcohol were found, but the relative energy barrier for the dissociation step was too great, therefore making subsequent $\mathrm{HCO} / \mathrm{HOC}$ radical formation unviable.

The reaction mechanisms between $\mathrm{O}\left({ }^{3} \mathrm{P}\right)+$ propargyl alcohol and $\mathrm{O}\left({ }^{3} \mathrm{P}\right)+3$-butyn-1-ol are proven to be similar through NRT analysis. For both reactions, NSD is distributed at a 1:3 (O:C) ratio for $\mathrm{HCO}$ radical formation and accumulates almost entirely on the $\mathrm{C}$ atom for $\mathrm{HOC}$ 
formation. In addition, the $\mathrm{NBO}$ between $\mathrm{C}$ and $\mathrm{O}$ for $\mathrm{HCO}$ radical is about 2.5, whereas the NBO between $\mathrm{C}$ and $\mathrm{O}$ for $\mathrm{HOC}$ is around 2.

Future work on the topic of substituted alkyne combustion includes further studies into the subsequent dissociation pathways of $\mathrm{HCO} / \mathrm{HOC}$ radical from all intermediate products formed after the initial dissociation in the reaction of $\mathrm{O}\left({ }^{3} \mathrm{P}\right)+3$-butyn-1-ol. In addition, a full NRT analysis of the subsequent dissociation pathways in the $\mathrm{O}\left({ }^{3} \mathrm{P}\right)+3$-butyn-1-ol reaction is also desirable to confirm the presence of an unpaired electron on the dissociated radical as well as bond breakage during the subsequent dissociation step.

The computational data presented in this project may offer some insight into the variations observed during the CRLAS studies. First, the reaction of $\mathrm{O}\left({ }^{3} \mathrm{P}\right)+$ propyne showed a relatively low intensity for the signature of $\mathrm{HCO}$ radical, whereas $\mathrm{O}\left({ }^{3} \mathrm{P}\right)+$ propargyl alcohol showed a strong signal. Buettner described two pathways for $\mathrm{HCO}$ radical formation in the $\mathrm{O}\left({ }^{3} \mathrm{P}\right)+$ propyne reaction, ${ }^{28}$ whereas this project reveals six pathways for $\mathrm{HCO} / \mathrm{HOC}$ radical formation in the $\mathrm{O}\left({ }^{3} \mathrm{P}\right)+$ propargyl alcohol reaction. The hypothesis given here may be described as a "statistical" explanation for the stronger HCO radical signal observed in the propargyl alcohol reaction. More possibilities for $\mathrm{HCO}$ radical formation in the propargyl alcohol reaction means that $\mathrm{HCO}$ radical is forming in higher abundance than in the propyne reaction. Second, the reaction of $\mathrm{O}\left({ }^{3} \mathrm{P}\right)+$ butyne showed a signal for $\mathrm{HCO}$ radical that was stronger than propyne, yet weaker than 3-butyn-1-ol. Buettner described two HCO radical formation pathways for the reaction of $\mathrm{O}\left({ }^{3} \mathrm{P}\right)+$ butyne, whereas this project shows six $\mathrm{HCO} / \mathrm{HOC}$ radical pathways for the reaction of $\mathrm{O}\left({ }^{3} \mathrm{P}\right)+3$-butyn-1-ol (including the viable subsequent $\mathrm{HCO}$ pathway). The combination of more possibilities for $\mathrm{HCO}$ dissociation and the presence of a viable subsequent HCO radical formation pathway may explain the stronger HCO radical signal observed for 3- 
butyn-1-ol over butyne during the CRDLAS experiments. Building upon the work conducted by Buettner and Dilday has revealed new insights into the formation of HCO radical from the combustion of hydroxyl-substituted alkynes. 


\section{REFERENCES}

1) U.S. Energy Information Administration / Primary Energy Consumption by Source and Sector, http://www.eia.gov/totalenergy/data/monthly/pdf/flow/primary_energy.pdf (accessed 10/9/16).

2) Total Energy Data Browser, https://www.eia.gov/totalenergy/data/browser/\#/?f=A\&start=1949\&end=2016\&charted= $\underline{4-6-7-14}($ accessed 10/9/16).

3) NASA Climate Change, http://climate.nasa.gov/ (accessed 10/9/16).

4) Andresen, P.; Luntz, A. C. The chemical dynamics of the reactions of $\mathrm{O}\left({ }^{3} \mathrm{P}\right)$ with saturated hydrocarbons. I. Experiment. J. Chem. Phys. 72, 5842-5850 (1980).

5) Herron, J. T. Evaluated Chemical Kinetic Data for the Reactions of Atomic Oxygen $\mathrm{O}\left({ }^{3} \mathrm{P}\right)$ with Saturated Organic Compounds in the Gas Phase. J. Phys. Chem. 17, 967-1026 (1988).

6) Ausfelder, F.; McKendrick, K. G. The dynamics of reactions of $\mathrm{O}\left({ }^{3} \mathrm{P}\right)$ atoms with saturated hydrocarbons and related compounds. Science Reviews 25, 299-370 (2000).

7) Fu, B.; Han, Y.; Bowman, J. M.; Leonori, F.; Balucani, N.; Angelucci, L.; Occhiogrosso, A.; Petrucci, R.; Casavecchia, P. Experimental and theoretical studies of the $\mathrm{O}\left({ }^{3} \mathrm{P}\right)+$ $\mathrm{C}_{2} \mathrm{H}_{4}$ reaction dynamics: collision energy dependence of branching ratios and extent of intersystem crossing. J. Chem. Phys. 137, 22A532 (2012).

8) West, A. C.; Kretchmer, J. S.; Sellner, B.; Park, K.; Hase, W. L.; Lischka, H.; Windus, T. L. $\mathrm{O}\left({ }^{3} \mathrm{P}\right)+\mathrm{C}_{2} \mathrm{H}_{4}$ potential energy surface: study at the multireference level. J. Phys. Chem. A 113, 12663-12674 (2009). 
9) Saito, T.; Nishihara, S.; Kataoka, Y.; Nakanishi, Y.; Kitagawa, Y.; Kawakami, T.; Yamanaka, S.; Okumura, M.; Yamaguchi, K. Reinvestigation of the reaction of ethylene and singlet oxygen by the approximate spin projection model. Comparison with multireference coupled-cluster calculations. J. Phys. Chem. A 114, 7967-7974 (2010).

10) Leonori, F.; Balucani, N.; Nevrly, V.; Bergeat, A.; Falcinelli, S.; Vanuzzo, G.; Casavecchia, P. Experimental and theoretical studies on the dynamics of the $\mathrm{O}\left({ }^{3} \mathrm{P}\right)+$ propene reaction: primary products, branching ratios, and role of intersystem crossing. $J$. Phys. Chem. A 119, 14632-14652 (2015).

11) Cavallotti, C. Relevance of the channel leading to formaldehyde + triplet ethylidene in the $\mathrm{O}\left({ }^{3} \mathrm{P}\right)+$ propene reaction under combustion conditions. J. Phys. Chem. A 5, 42134218 (2014).

12) Zhang, W.; Du, B.; Feng, C. An ab initio dynamics study on the reaction of $\mathrm{O}\left({ }^{3} \mathrm{P}\right)$ with $\mathrm{CH}_{3} \mathrm{CH}=\mathrm{CH}_{2}\left({ }^{1} \mathrm{~A}^{\prime}\right)$. J. Mol. Struc.: THEOCHEM 806, 121-129 (2007).

13) Huang, X.; Xing, G.; Bersohn, R. Dynamics of the reaction of $\mathrm{O}\left({ }^{3} \mathrm{P}\right)$ atoms with acetylene. J. Phys. Chem. 101, 5818-5823 (1992).

14) Rajak, K.; Maiti, B. Communcations: direct dynamics study of the $\mathrm{O}\left({ }^{3} \mathrm{P}\right)+\mathrm{C}_{2} \mathrm{H}_{4}$ reaction: contribution from spin nonconserving route. J. Chem. Phys. 133, 011101 (2010).

15) Harding, L. B. Theoretical studies on the reaction of atomic oxygen $\left({ }^{3} \mathrm{P}\right)$ with acetylene. J. Phys. Chem. 85, 10-11 (1981).

16) Girard, Y.; Chaquin, $\mathrm{P}$. Addition reaction of ${ }^{1} \mathrm{D}$ and ${ }^{3} \mathrm{P}$ atomic oxygen with acetylene. Potential energy surfaces and stability of the primary products. Is oxirene only a triplet molecule? A theoretical study. J. Phys. Chem. 107, 10462-10470 (2003). 
17) Nguyen, T. L.; Vereechken, L.; Peeters, J. Quantum chemical and theoretical kinetics study of the $\mathrm{O}\left({ }^{3} \mathrm{P}\right)+\mathrm{C}_{2} \mathrm{H}_{4}$ reaction: a multistate process. J. Phys. Chem. 110, 6696-6706 (2006).

18) Zhao, S.; Wu, W.; Zhao, H.; Wang, H.; Yang, C.; Liu, K.; Su, H. Adiabatic and nonadiabatic reaction pathways of the $\mathrm{O}\left({ }^{3} \mathrm{P}\right)$ with propyne. J. Phys. Chem. A 113, 23-24 (2009).

19) Savee, J. D.; Borkar, S.; Welz, O.; Sztáray, B.; Taatjes, C.; Osborn, D. L. Multiplexed photoionization mass spectrometry investigation of the $\mathrm{O}\left({ }^{3} \mathrm{P}\right)+$ propyne reaction. J. Phys. Chem. A. 119, 7388-7403 (2015).

20) Vanuzzo, G.; Balucani, N.; Leonori, F.; Stranges, D.; Falcinelli, S.; Bergeat, A.; Casavecchia, P.; Gimondi, I.; Cavallotti, C. Isomer-specific chemistry in the proyne and allene reaction with oxygen atoms: $\mathrm{CH}_{3} \mathrm{CH}+\mathrm{CO}$ versus $\mathrm{CH}_{2} \mathrm{CH}_{2}+\mathrm{CO}$ products. $J$. Phys. Chem. Lett. 7, 1010-1015 (2016).

21) Singleton, D. L.; Cvetanović. Temperature dependence of the reactions of oxygen atoms with olefins. J. Am. Chem. Soc. 98, 6812-19 (1976).

22) Neeb, P.; Horie, O.; Moortgat, G. The ethane-ozone reaction in the gas phase. J. Phys. Chem. 102, 6778-6785 (1998).

23) Quandt, R.; Min, Z.; Wang, X.; Bersohn, R. Reactions of O ( $\left.{ }^{3} \mathrm{P}\right)$ with alkenes: H, $\mathrm{CH}_{2} \mathrm{CHO}, \mathrm{CO}$, and $\mathrm{OH}$ channels. J. Phys. Chem. A 102, 60-64 (1998).

24) Vaidya, W. M. Spectrum of the flame of ethylene. Proc. R. Soc. London, Ser. A 147, 513521 (1934).

25) Min, Z.; Wong, T.; Quandt, R.; Bersohn R. The reactions of O $\left({ }^{3} \mathrm{P}\right)$ with alkenes: The formyl radical channel. J. Phys. Chem. 103, 10451-10453 (1999). 
26) Serrano-Andrés, L.; Forsberg, N.; Malmqvist, P. Vibronic structure in triatomic molecules: the hydrocarbon flame bands of the formyl radical (HCO). A theoretical study. J. Chem. Phys. 108, 7202-16 (1998).

27) Kinsey, J. L. Laser-induced fluorescence. Ann. Rev. Phys. Chem. 28, 349-372 (1977).

28) Buettner, A. D. M.S. Thesis, Illinois State University, 2010.

29) Dilday, B. J. M.S. Thesis, Illinois State University, 2014.

30) Casavecchia, P.; Leonori, F.; Balucani, N. Reaction dynamics of oxygen atoms with unsaturated hydrocarbons from crossed molecular beam studies: primary products, branching ratios and role of intersystem crossing. Int. Rev. Phys. Chem. 34, 161-204 (2015).

31) Scherer, J. J.; Paul, J. B.; O’Keefe, A.; Saykally, R. J. Cavity ringdown laser absorption spectroscopy: history, development, and application to pulsed molecular beams. Chem. Rev. 97, 25-51 (1997).

32)R, Statistical Language Program, https://www.r-project.org (accessed 10/25/16).

33) Gaussian 09, Frisch, M. J.; Trucks, G. W.; Schlegel, H. B.; Scuseria, G. E.; Robb, M. A.; Cheeseman, J. R.; Scalmani, G.; Barone, V.; Mennucci, B.; Petersson, G. A.; Nakatsuji, H.; Caricato, M.; Li, X.; Hratchian, H. P.; Izmaylov, A. F.; Bloino, J.; Zheng, G.; Sonnenberg, J. L.; Hada, M.; Ehara, M.; Toyota, K.; Fukuda, R.; Hasegawa, J.; Ishida, M.; Nakajima, T.; Honda, Y.; Kitao, O.; Nakai, H.; Vreven, T.; Montgomery, J. A., Jr.; Peralta, J. E.; Ogliaro, F.; Bearpark, M.; Heyd, J. J.; Brothers, E.; Kudin, K. N.; Staroverov, V. N.; Kobayashi, R.; Normand, J.; Raghavachari, K.; Rendell, A.; Burant, J. C.; Iyengar, S. S.; Tomasi, J.; Cossi, M.; Rega, N.; Millam, N. J.; Klene, M.; Knox, J. E.; Cross, J. B.; Bakken, V.; Adamo, C.; Jaramillo, J.; Gomperts, R.; Stratmann, R. E.; 
Yazyev, O.; Austin, A. J.; Cammi, R.; Pomelli, C.; Ochterski, J. W.; Martin, R. L.; Morokuma, K.; Zakrzewski, V. G.; Voth, G. A.; Salvador, P.; Dannenberg, J. J.;

Dapprich, S.; Daniels, A. D.; Farkas, Ö.; Foresman, J. B.; Ortiz, J. V.; Cioslowski, J.;

Fox, D. J. Gaussian, Inc., Wallingford CT, 2009.

34) Harvey, J. N.; Aschi, M.; Schwarz, H.; Koch, W. The singlet and triplet states of phenyl cation. A hybrid approach for locating minimum energy crossing points between noninteracting potential energy surfaces. Theor. Chem. Acc. 99, 95-99 (1998).

35) Chachiyo, T.; Rodriguez, J. H. A direct method for locating minimum-energy crossing points (MECPs) in spin-forbidden transistions and nonadiabatic reactions. J. Chem. Phys. 123, 094711 (2005).

36) Foster, J. P.; Weinhold, F. Natural hybrid orbitals. J. Am. Chem. Soc. 102, 7211-7218 (1980).

37) Reed, A. E.; Weinhold, F. Natural bond orbital analysis of near-Hartree-Fock water dimer. J. Chem. Phys. 78, 4066-4073 (1983).

38) Reed, A. E.; Weinstock, R. B.; Weinhold, F. Natural population analysis. J. Chem. Phys. 83, 735-746 (1985).

39) Glendening, E. D.; Weinhold, F. Natural resonance theory: I. general formalism. $J$. Comp. Chem. 19, 593-609 (1998).

40) Carpenter, J. E.; Weinhold, F. Analysis of the geometry of the hydroxymethyl radical by the "different hybrids for different spins" natural bond orbital procedure. J. Mol. Struc.:THEOCHEM. 169, 41-62 (1988).

41) Kerr, J. A. Bond dissociation energies by kinetic methods. Chem. Rev. 66, 465-500 (1966). 
42) Quandt, R.; Min, Z.; Wang, X.; Bersohn, R. Reactions of $\mathrm{O}\left({ }^{3} \mathrm{P}\right)$ with alkenes: $\mathrm{H}$, $\mathrm{CH}_{2} \mathrm{CHO}, \mathrm{CO}$, and $\mathrm{OH}$ channels. J. Phys. Chem. 102, 60-64 (1998).

43) Xing G.; Huang, X.; Wang, X. Bersohn, R. Reactions of $\mathrm{O}\left({ }^{3} \mathrm{P}\right)$ with alkynes: the $\mathrm{CO}$ and H atom channels. J. Chem. Phys. 105, 488-95 (1996). 


\title{
APPENDIX A: ALL OPTIMIZED STRUCTURES FOR O $\left({ }^{3} \mathrm{P}\right)+$ PROPARGYL ALCOHOL AND O $\left({ }^{3} \mathrm{P}\right)+3$-BUTYN-1-OL AT THE M06-2X/CC-PVTZ LEVEL
}

\begin{abstract}
All structures from the $\mathrm{O}\left({ }^{3} \mathrm{P}\right)+$ propargyl alcohol and $\mathrm{O}\left({ }^{3} \mathrm{P}\right)+3$-butyn-1-ol reactions are presented here with potential energies listed in hartrees. All structures presented here were optimized at the M06-2x/cc-pVTZ level of theory. Energies listed here were collected via singlepoint calculations at the $\operatorname{CCSD}(\mathrm{T}) / \mathrm{cc}-\mathrm{pVTZ}$ level of theory, corrected with vibrational zero-point energies found at the M06-2x/cc-pVTZ level. Several species have multiple conformers listed; the lowest energy conformer was chosen for constructing the PEP and was kept consistent throughout the pathway. For example, IM-1T from the established HCO radical pathway for $\mathrm{O}\left({ }^{3} \mathrm{P}\right)+$ propargyl alcohol has 5 different conformers, with "cis-b" being the lowest in energy. So, isomer cis-b was chosen for IM-1T and each following species in the pathway has the "cis-b" conformation. Each panel in Appendix A shows a transition state in the center with reactants, products, or intermediates on either side to which the transition state connects.
\end{abstract}


M06-2x/cc-pVTZ Structures for the Triplet State Surface of $O\left({ }^{3} P\right)+$ Propargyl Alcohol

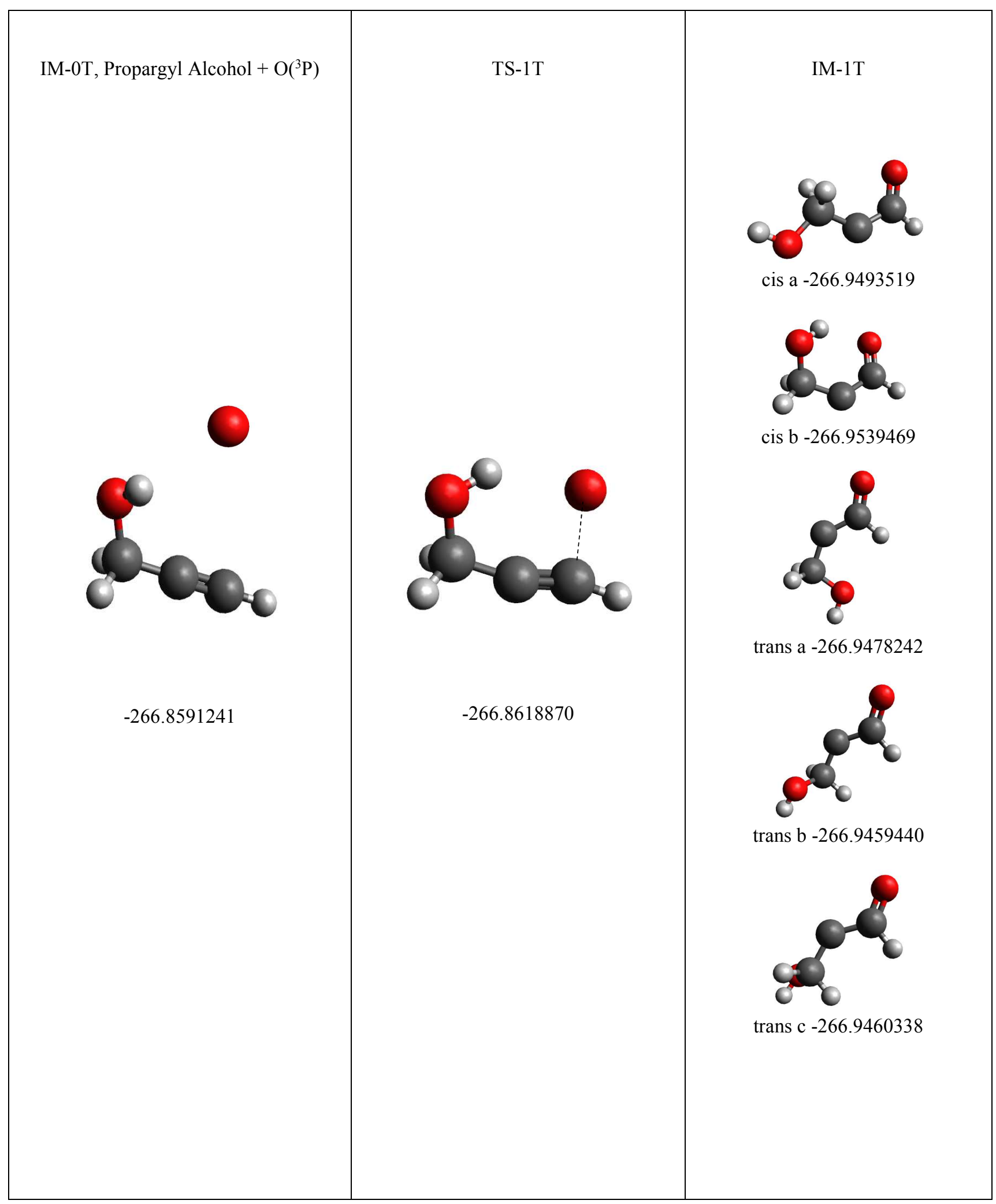




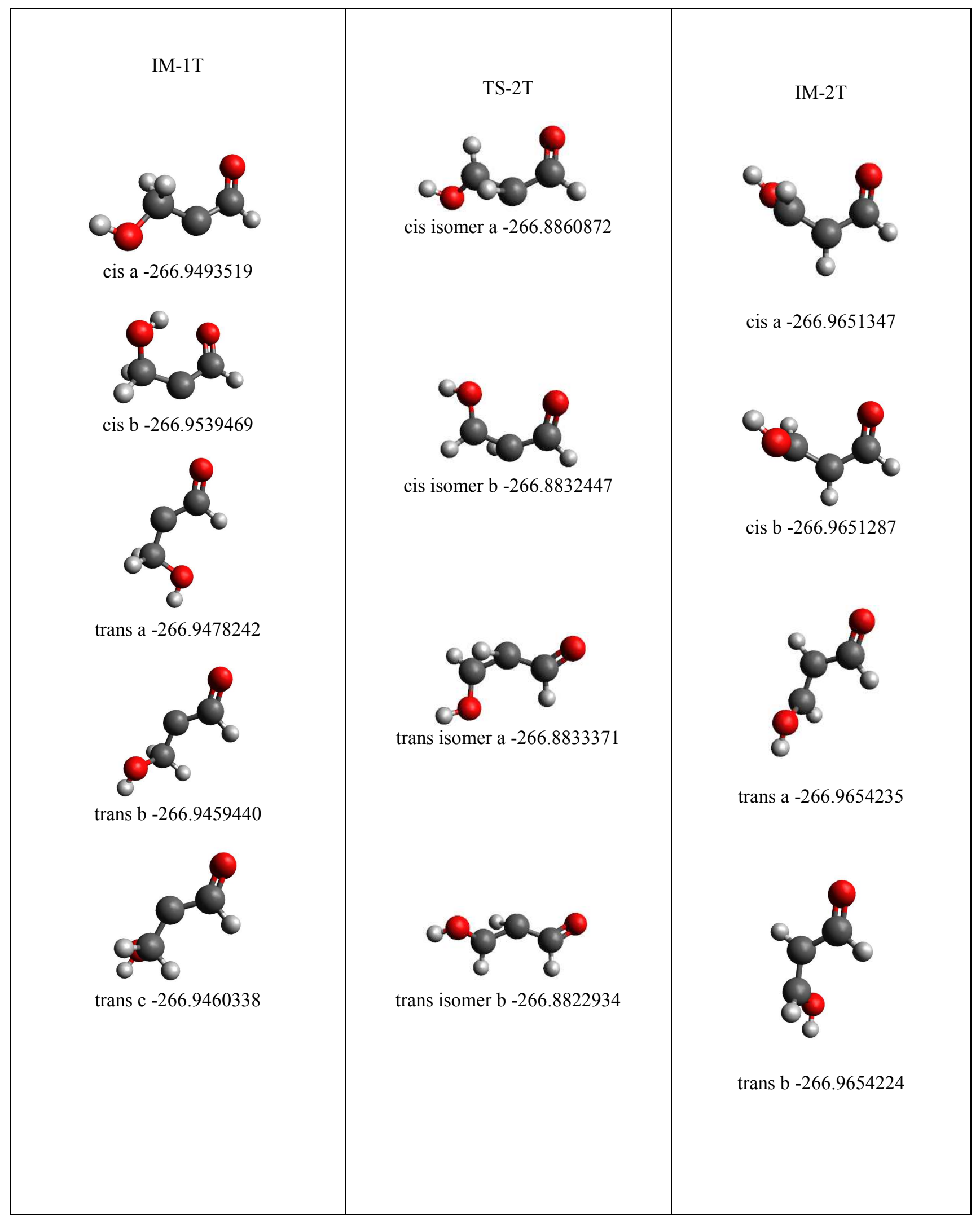




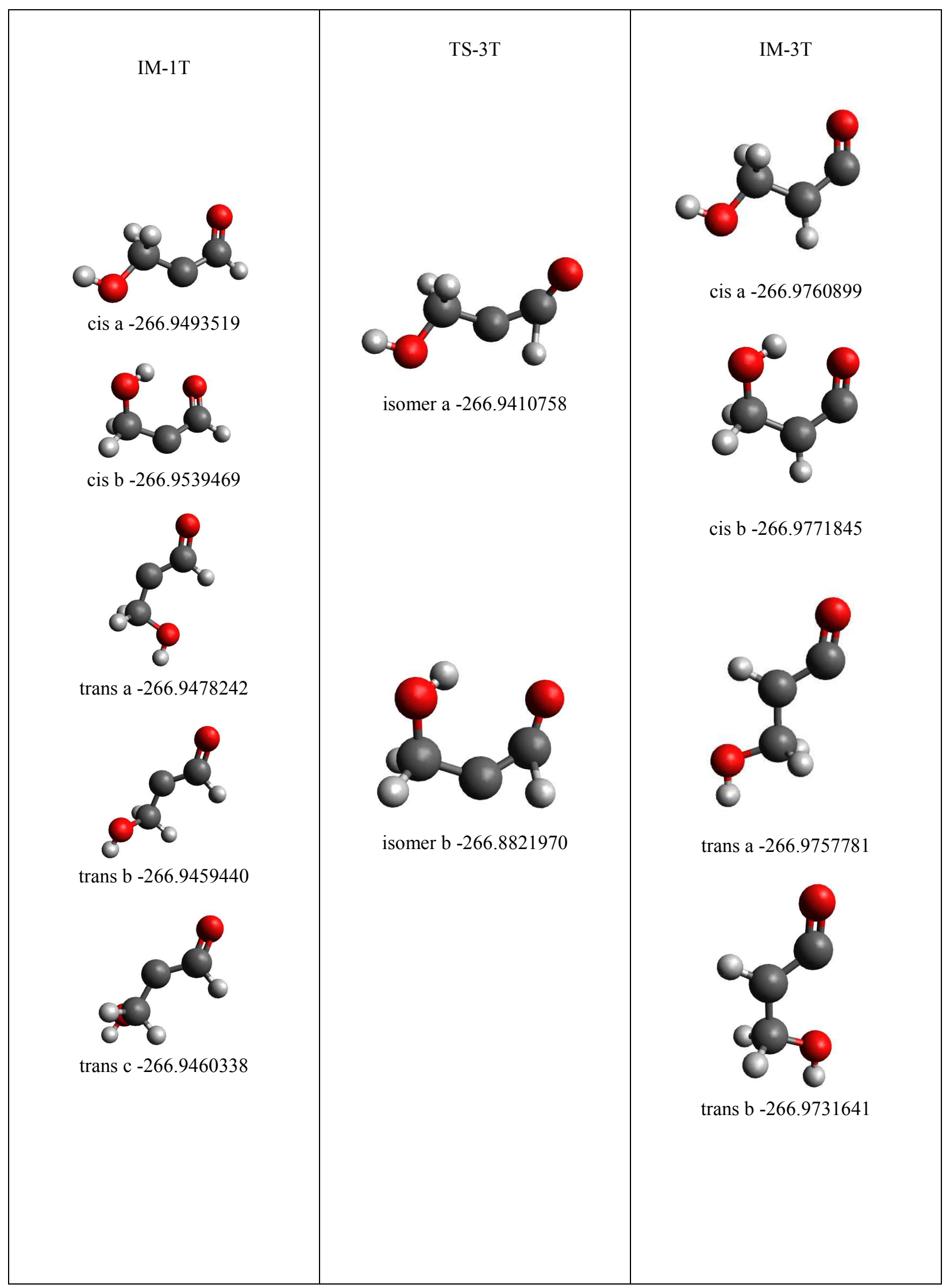




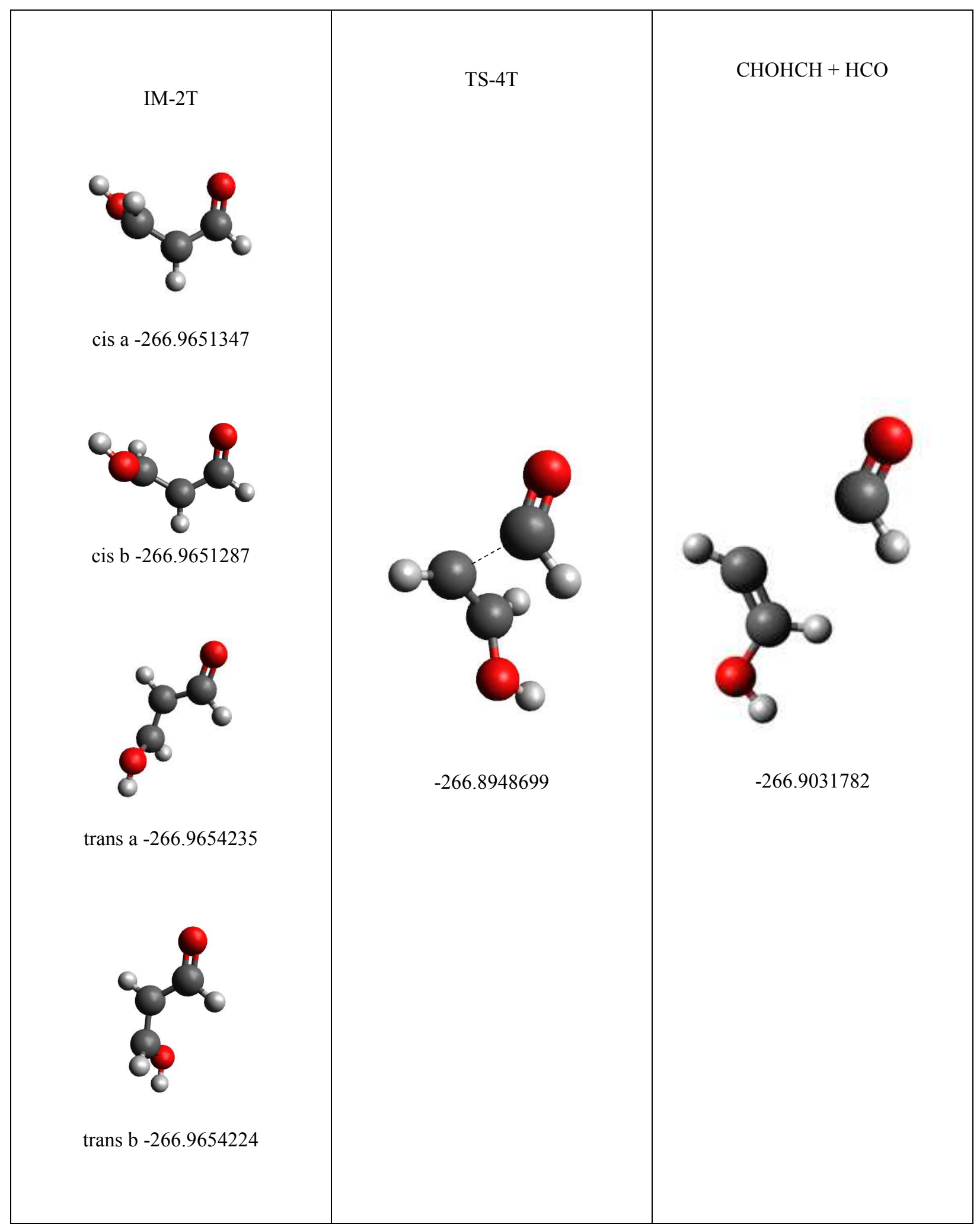




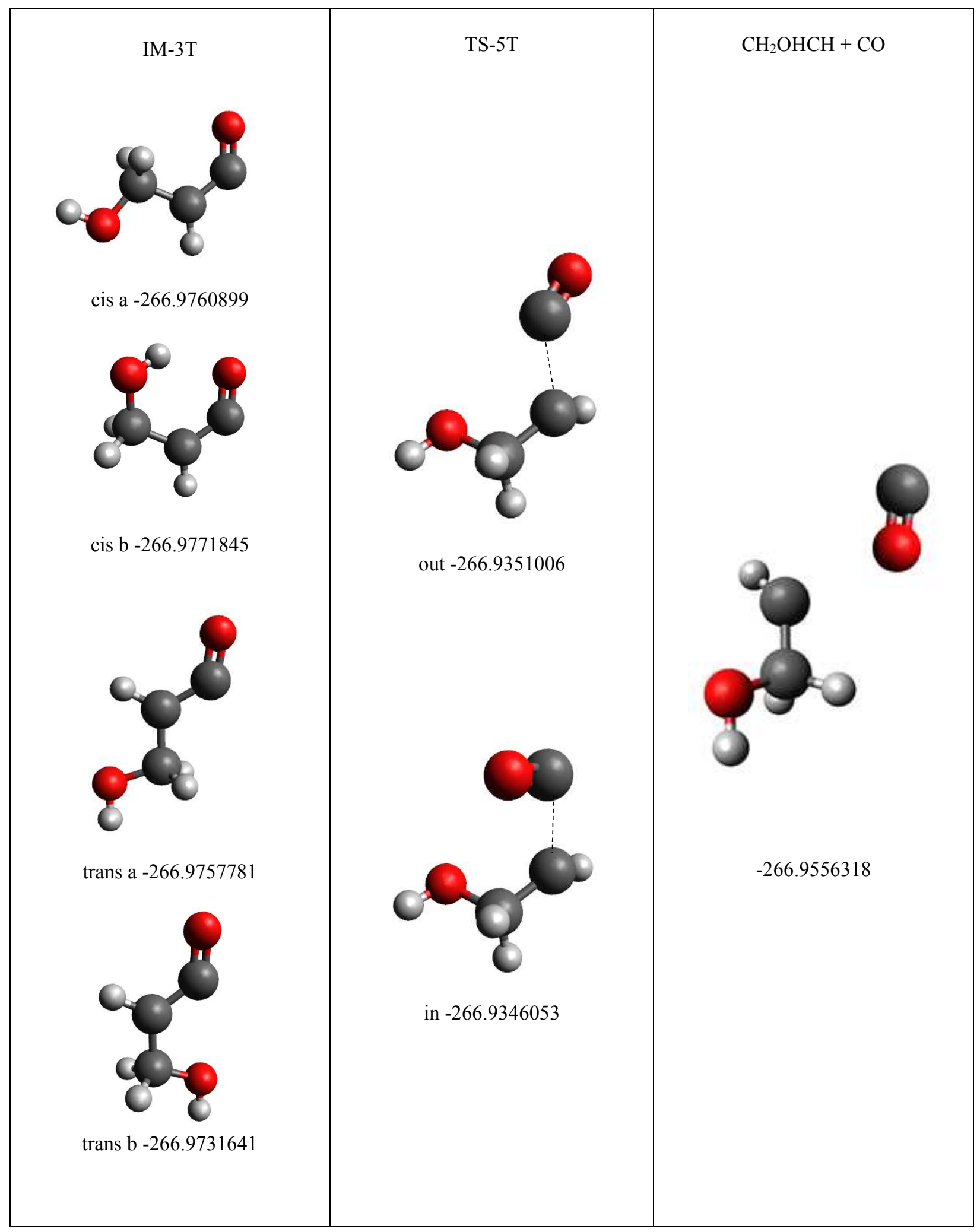




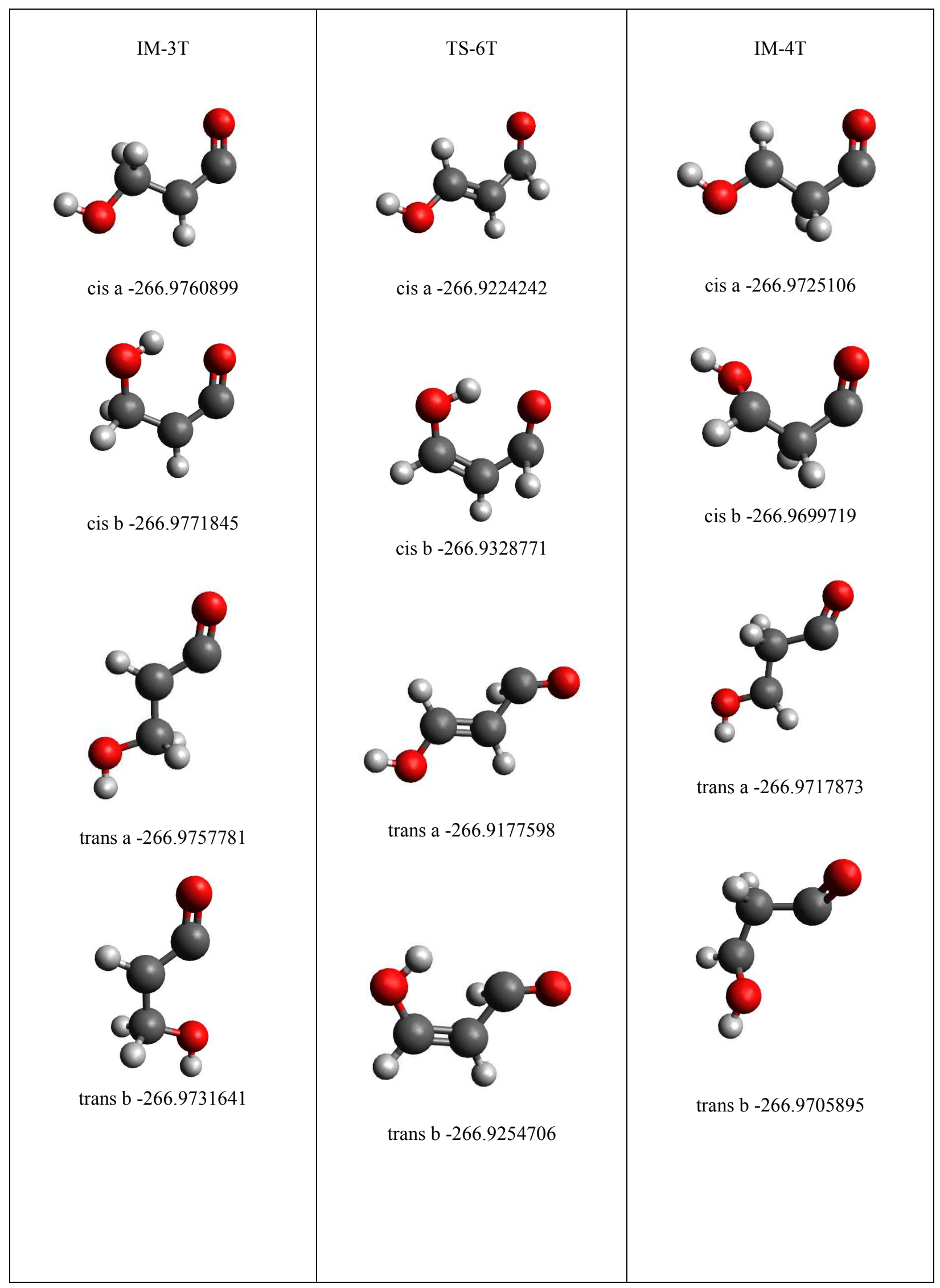




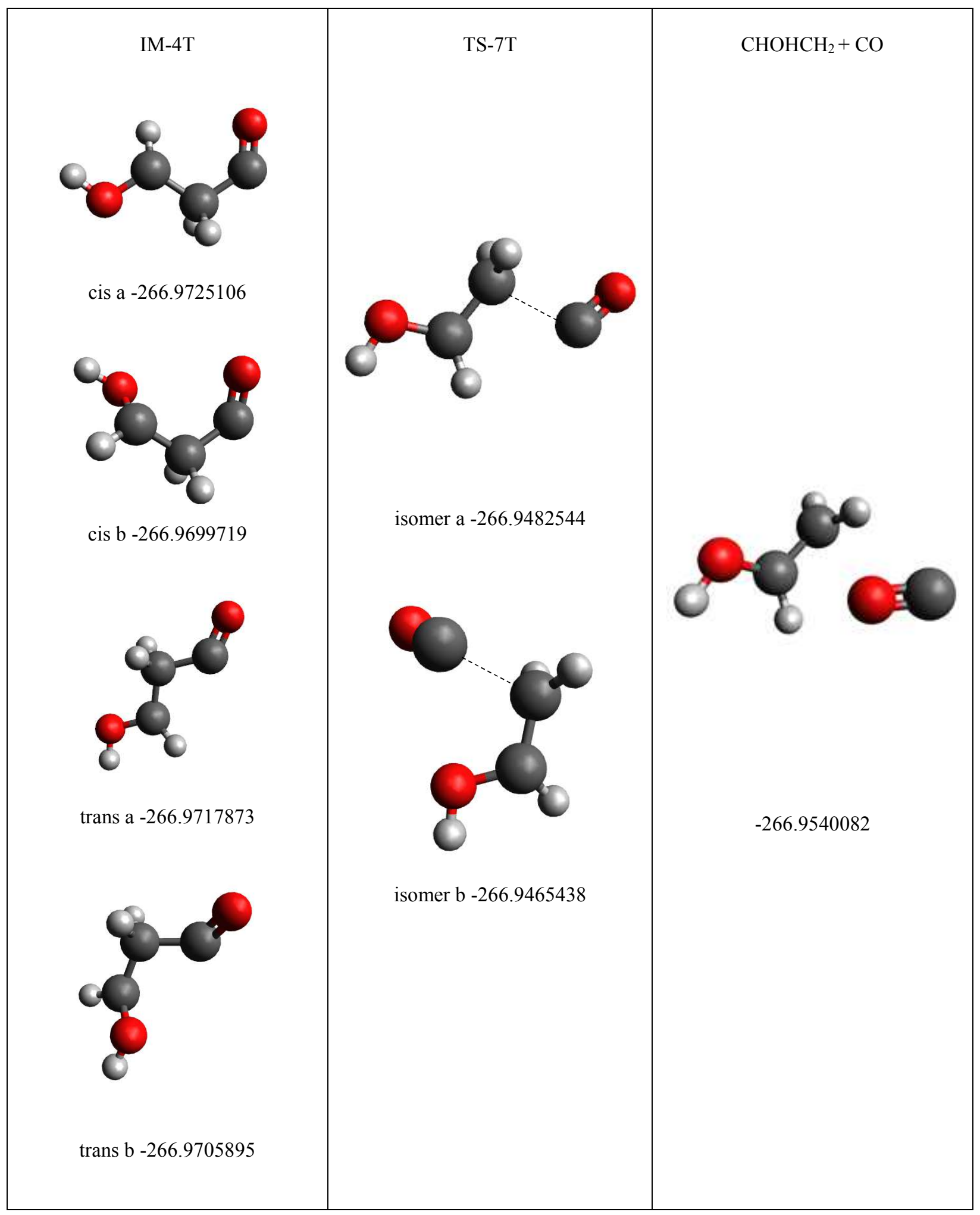


M06-2x/cc-pVTZ Structures for the Singlet State Surface of O $\left({ }^{3} P\right)+$ Propargyl Alcohol

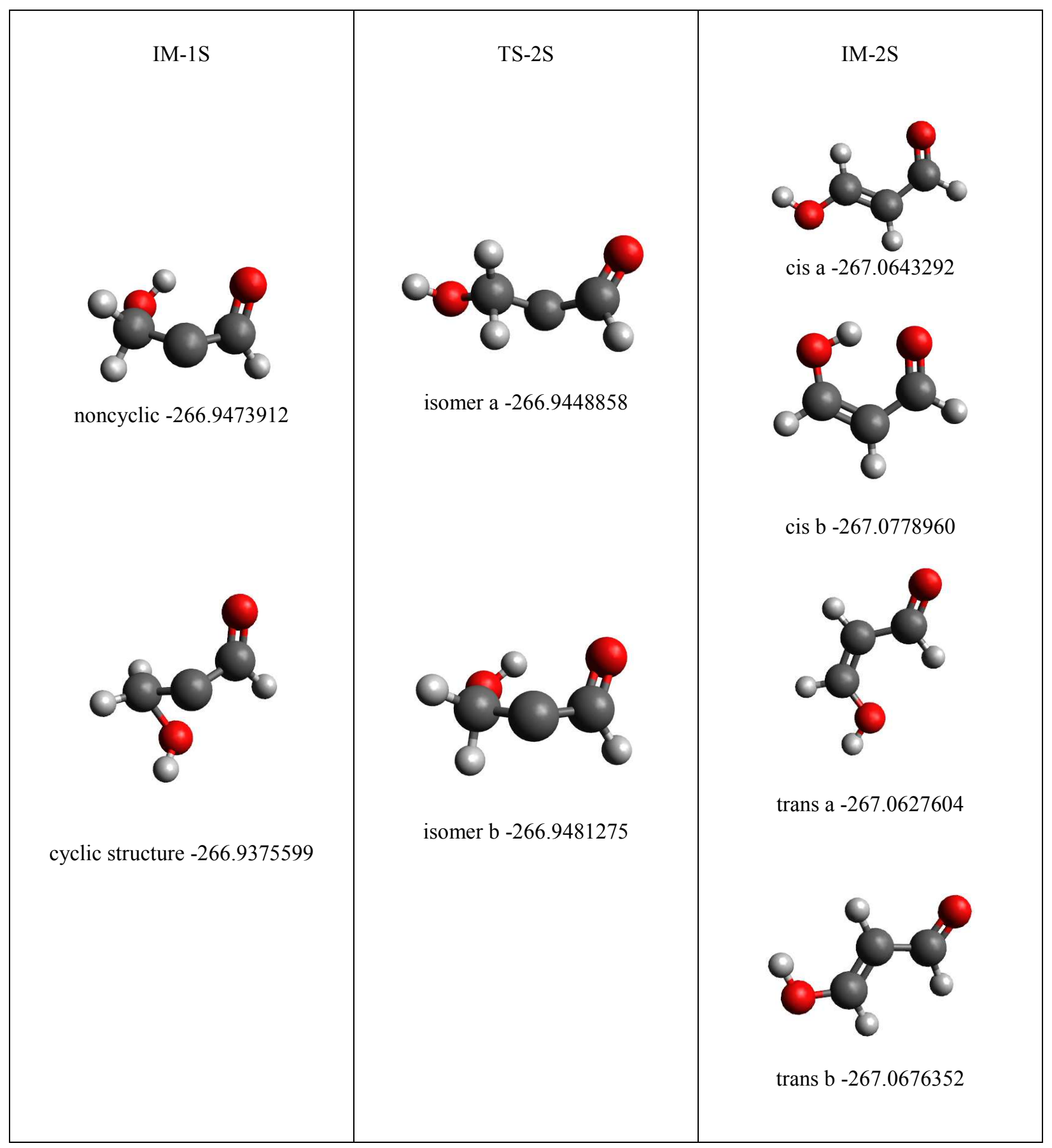




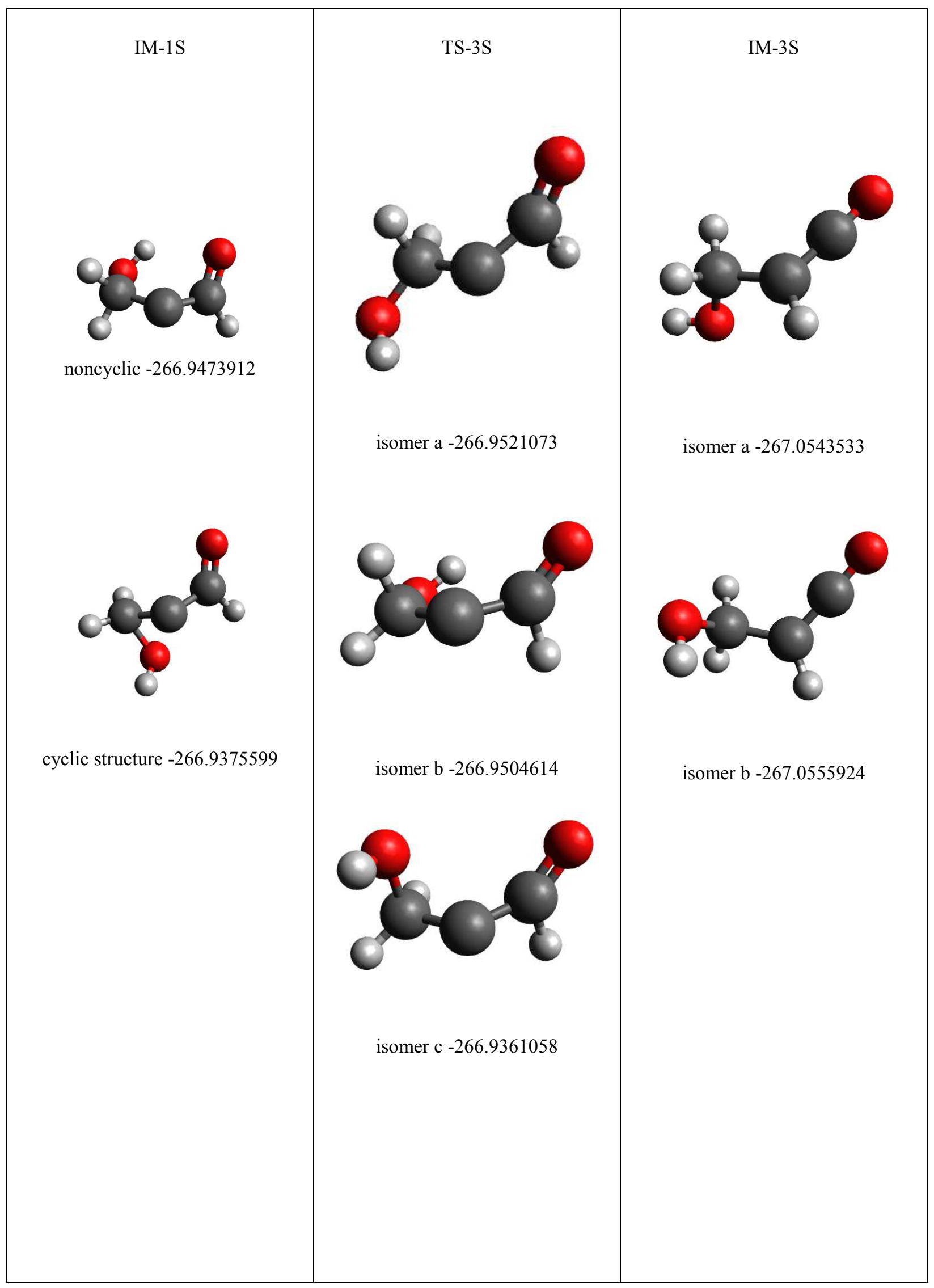




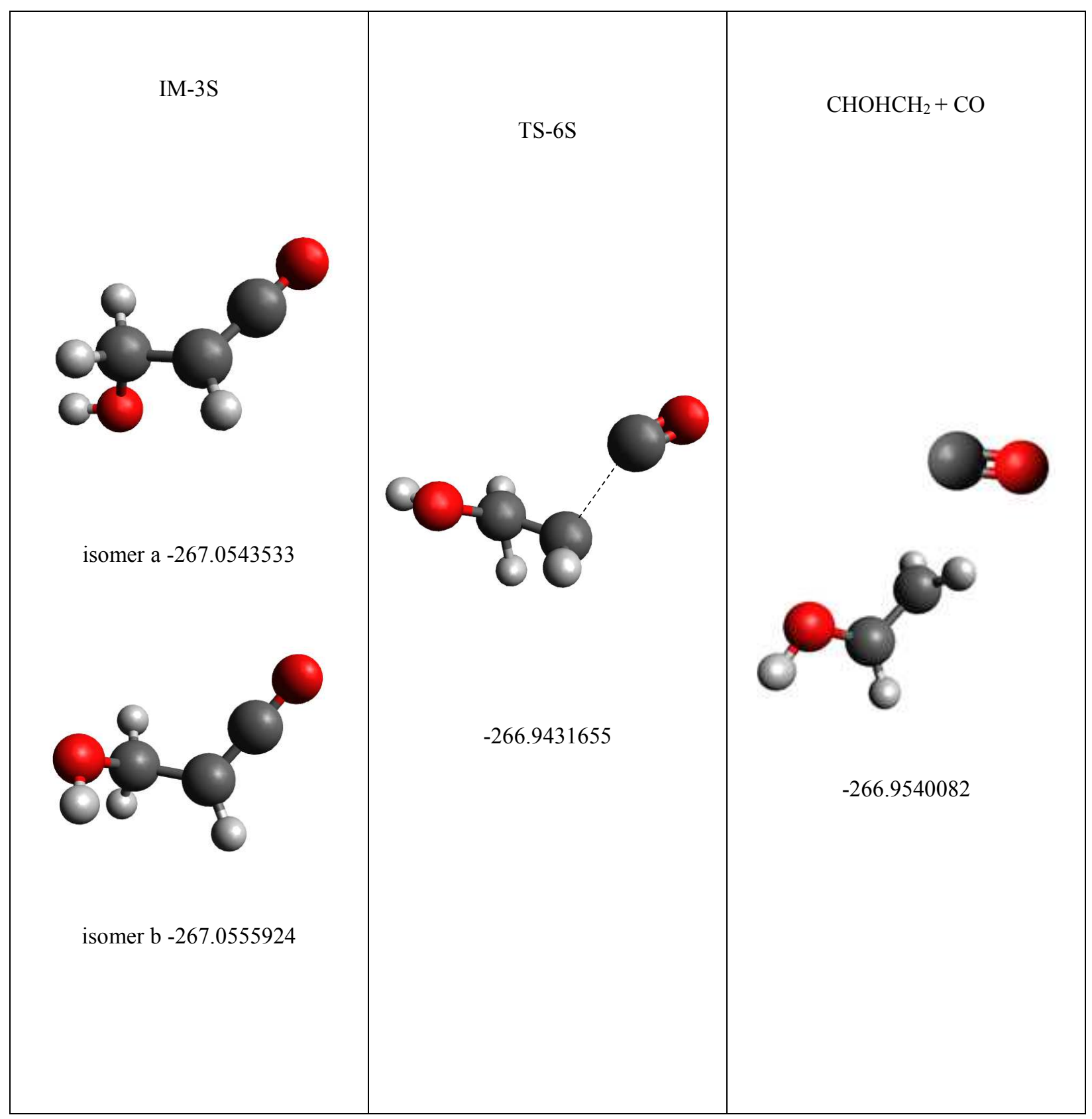




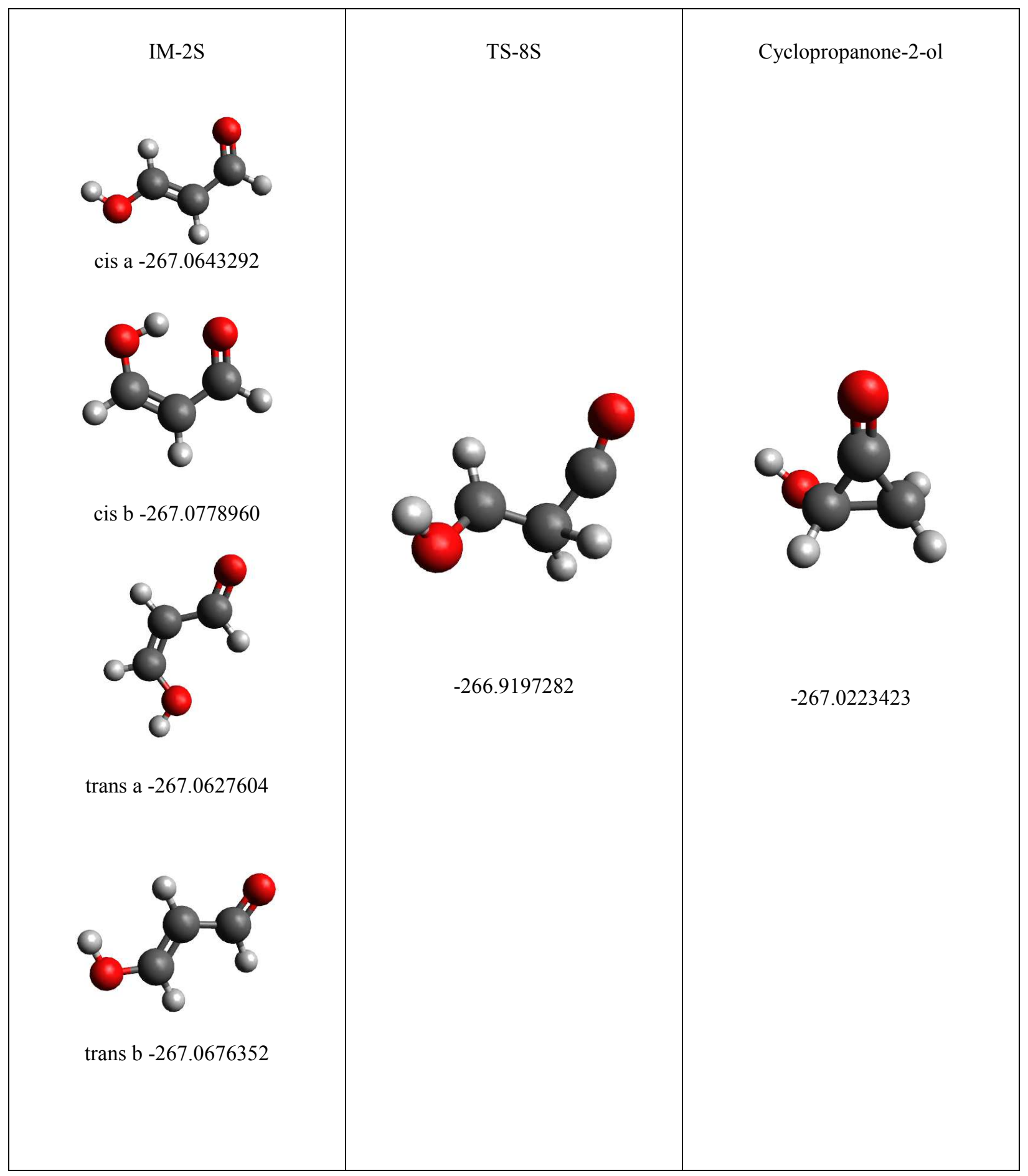




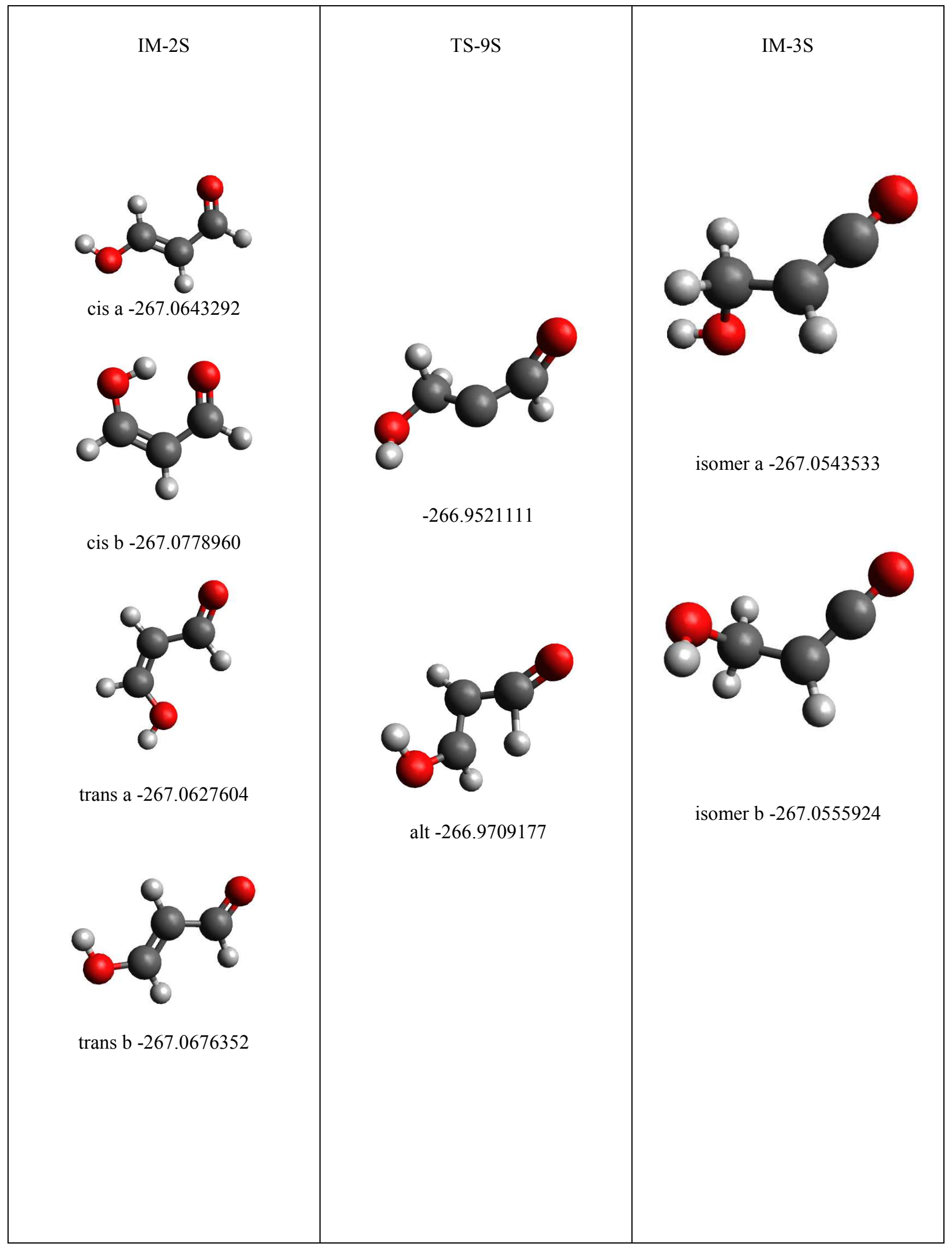




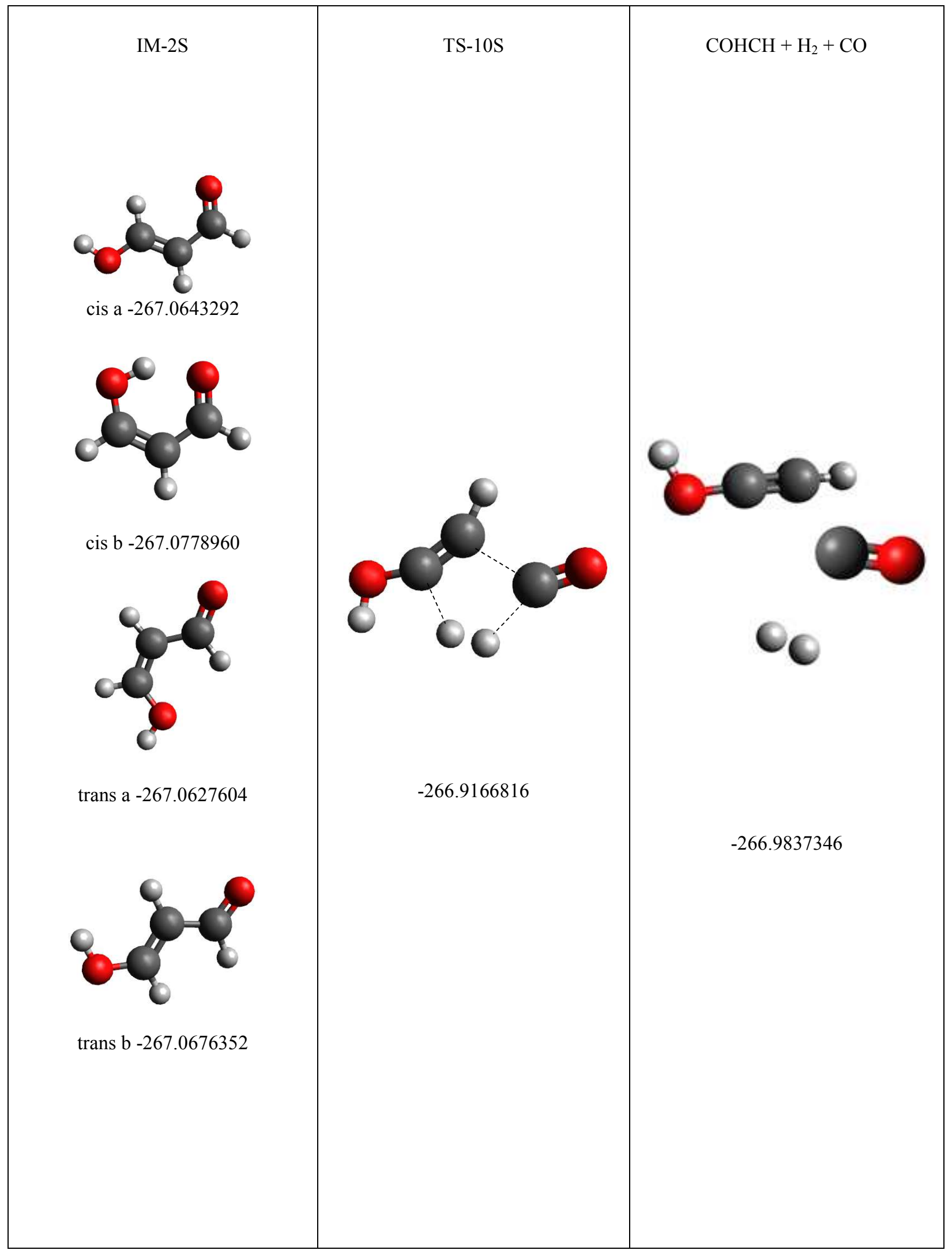




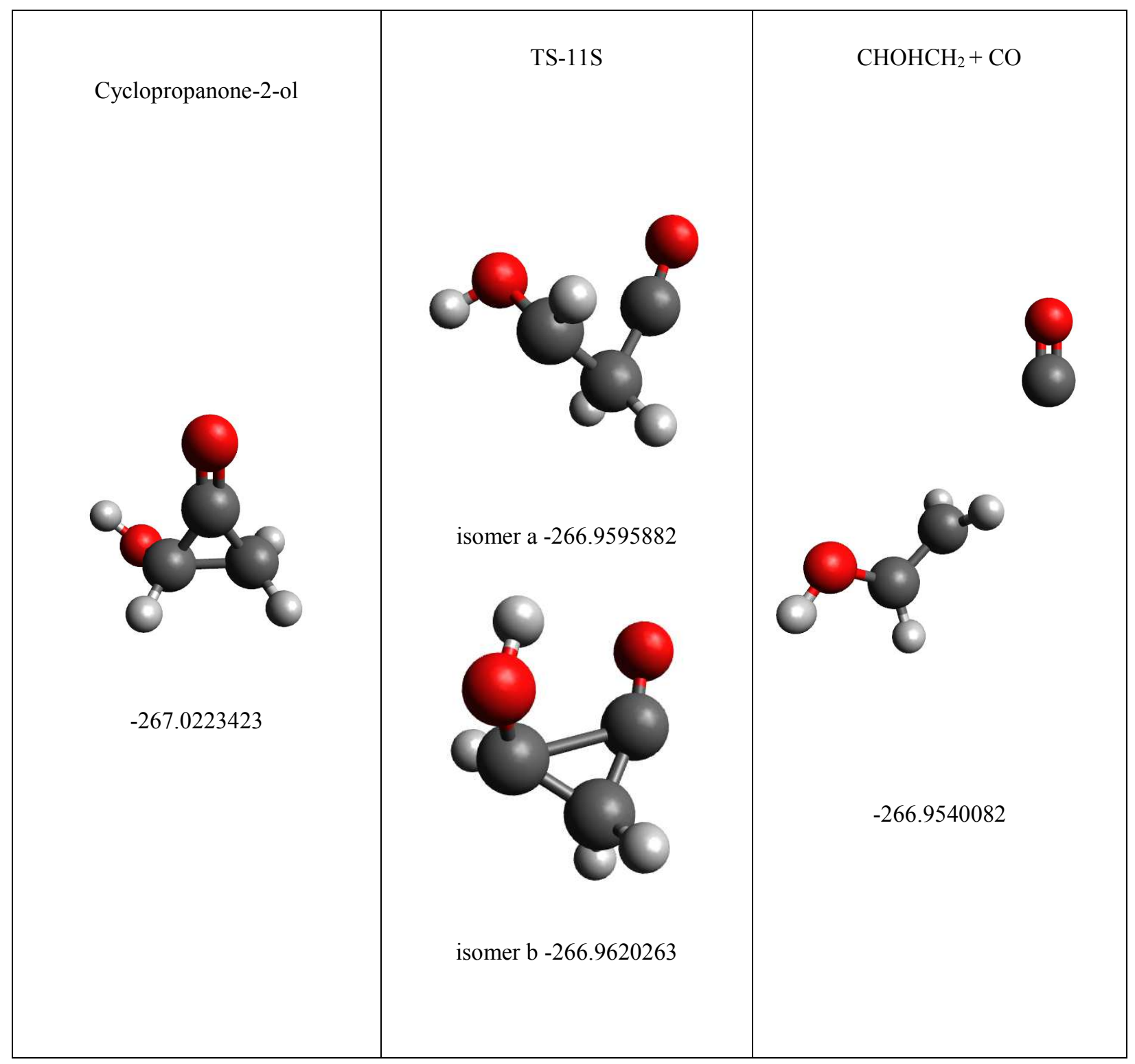


M06-2x/cc-pVTZ Structures for the Novel HCO/HOC Pathways, Triplet Surface of $\mathrm{O}\left({ }^{3} \mathrm{P}\right)+$ Propargyl Alcohol

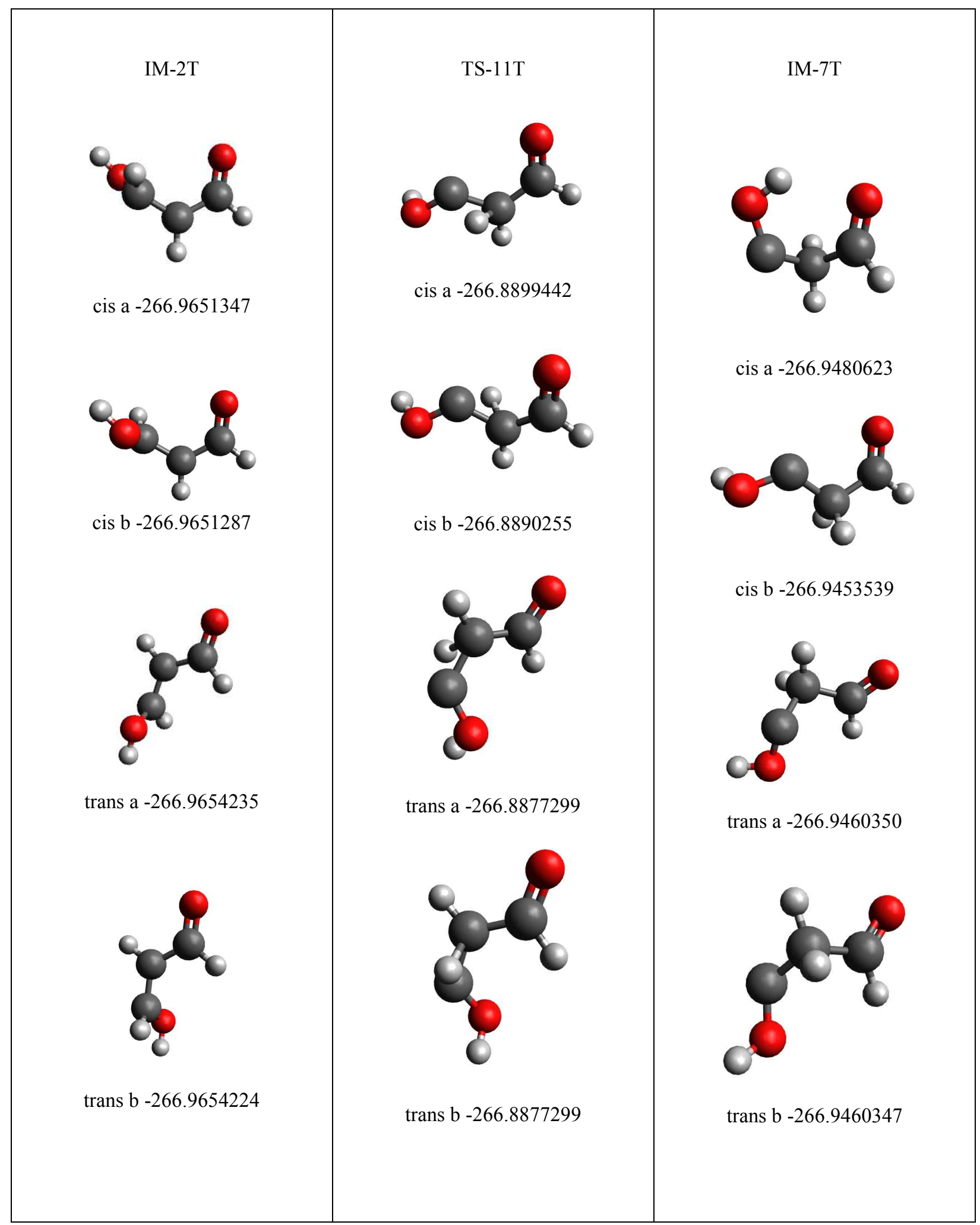




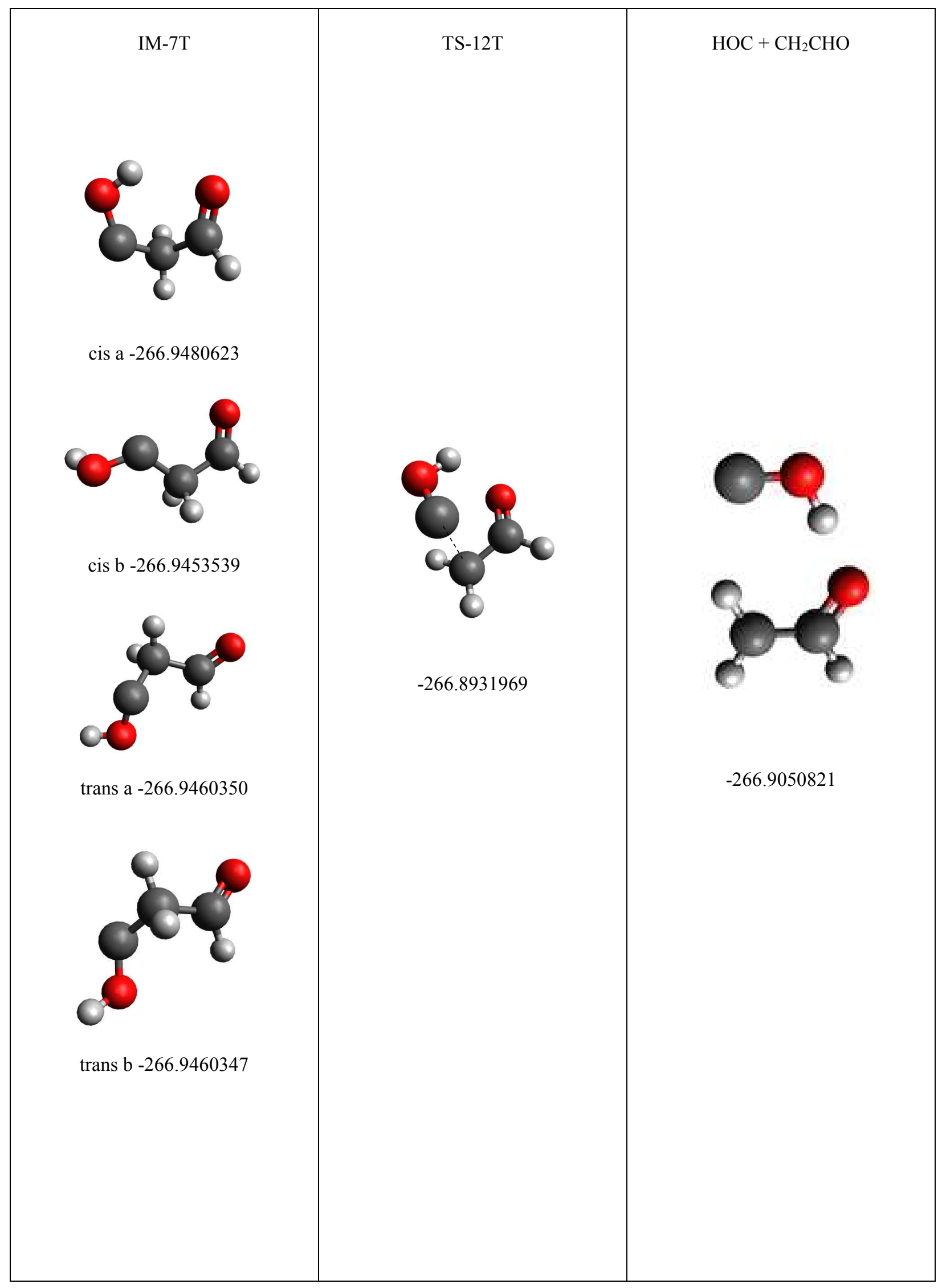




\begin{tabular}{|l|l|l|l|}
\hline HCO & TS-HCOtoHOC & HOC \\
\hline-113.8340068 & -113.7337104 & \\
\hline
\end{tabular}




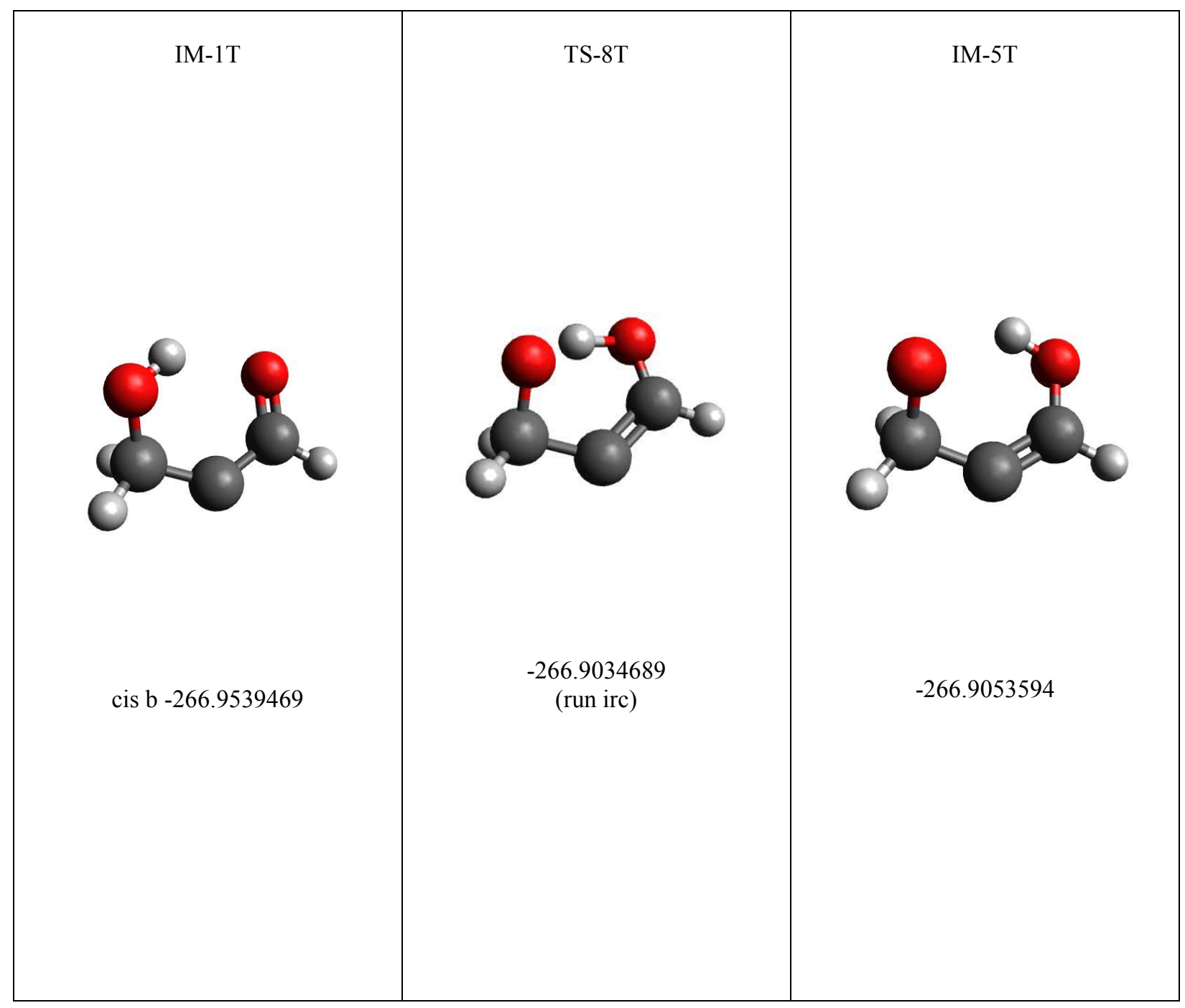




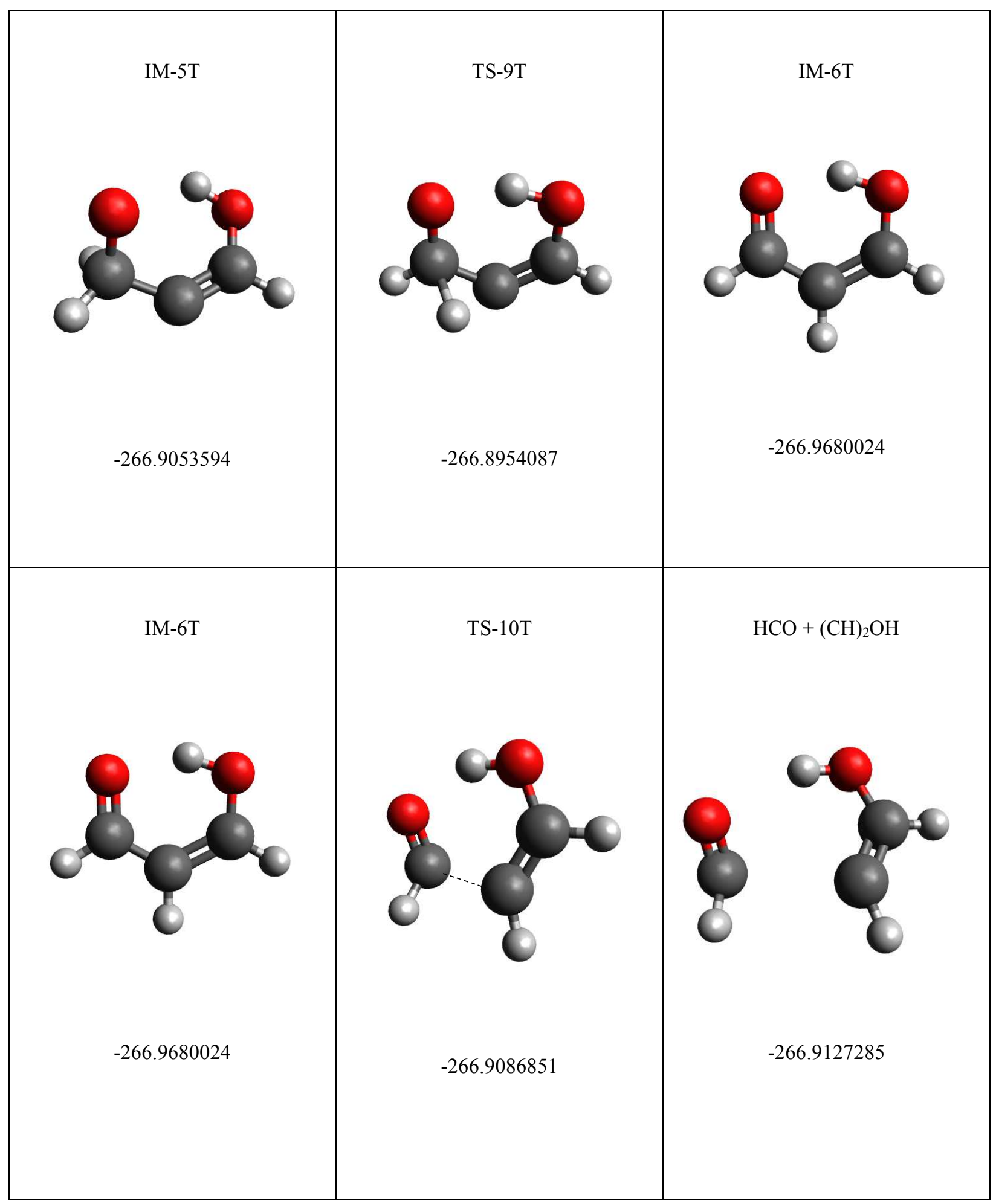




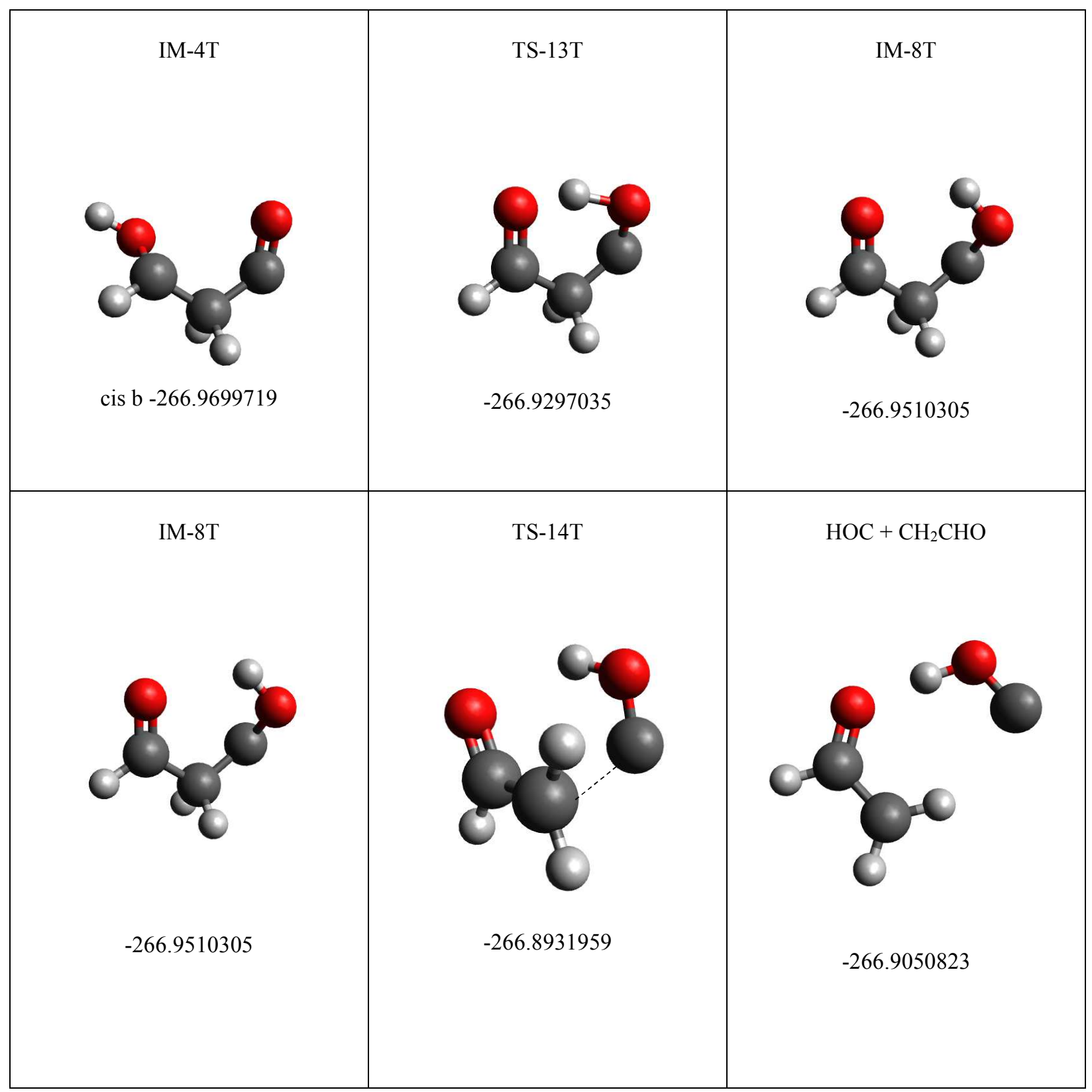




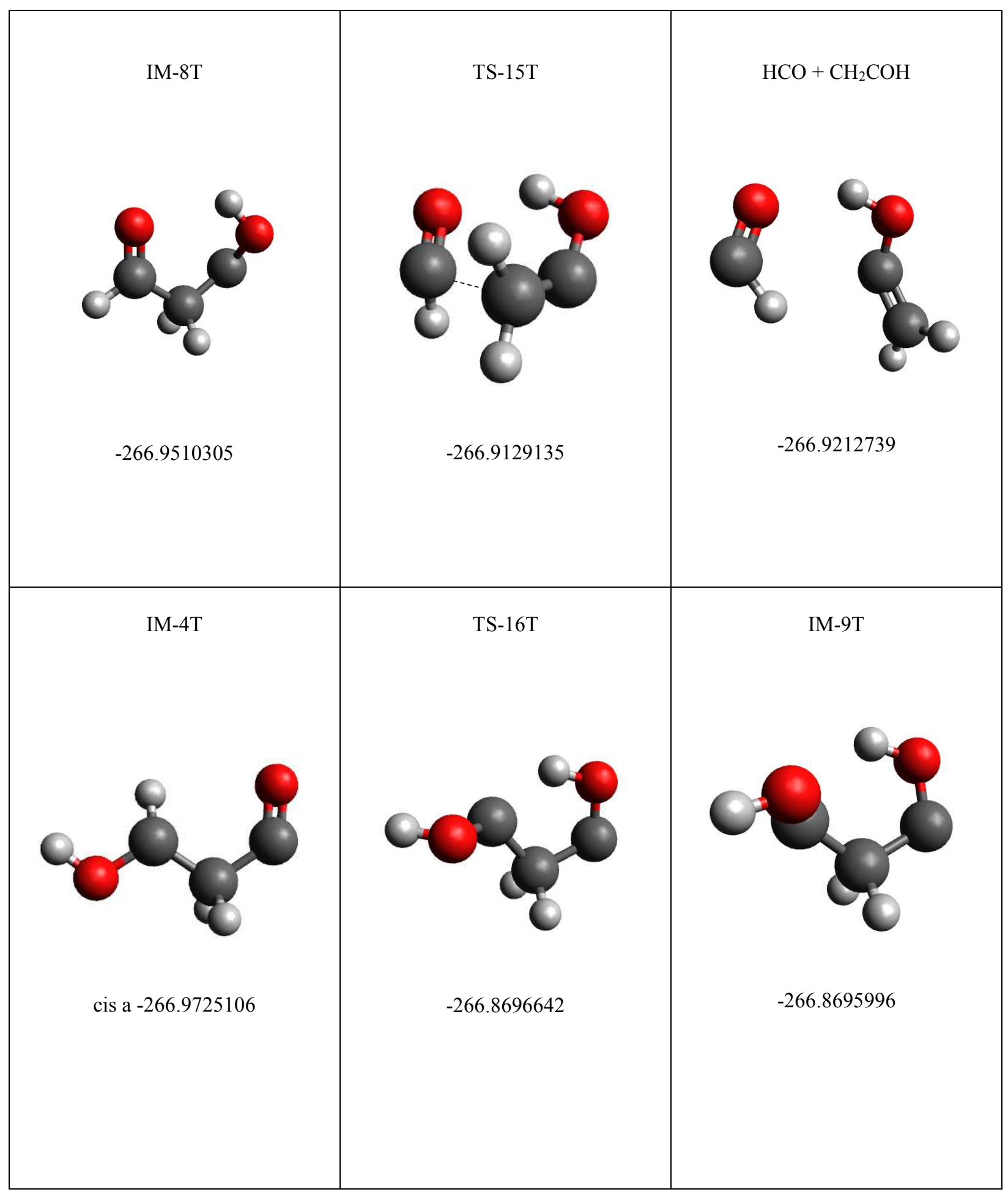




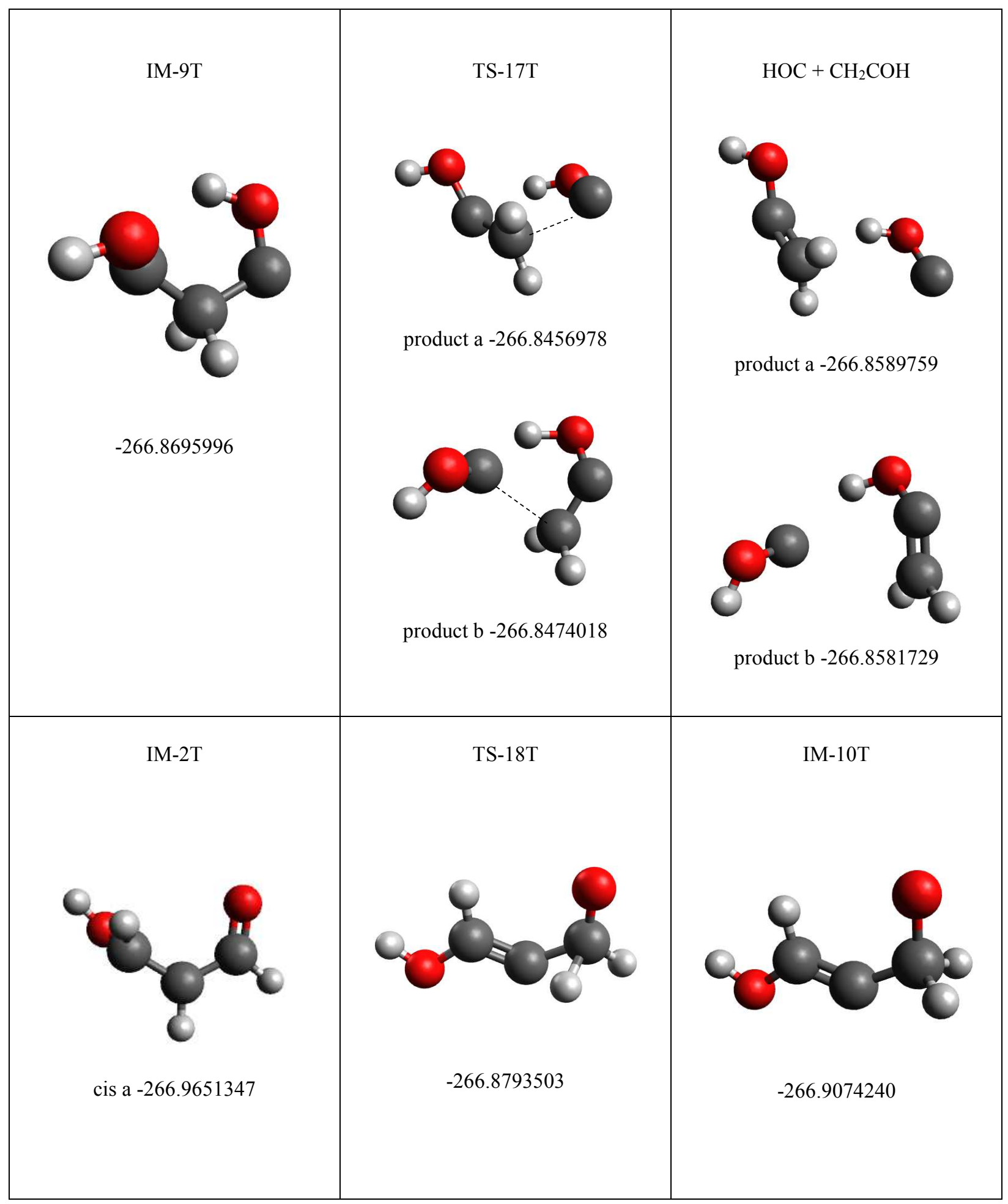




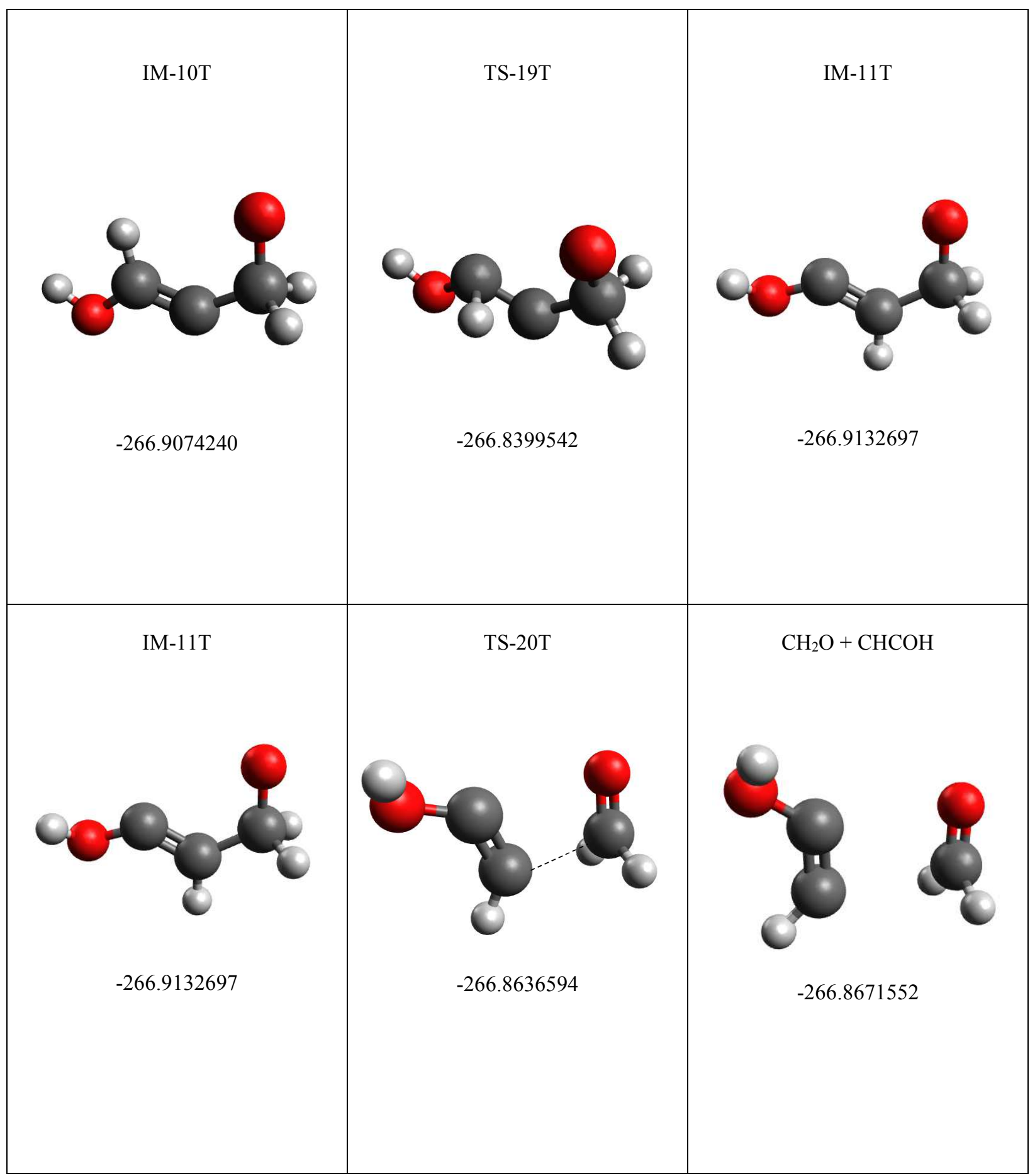




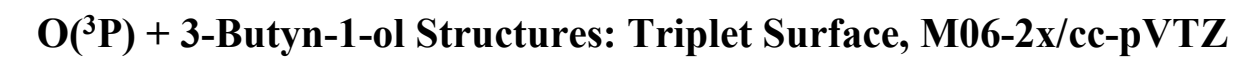

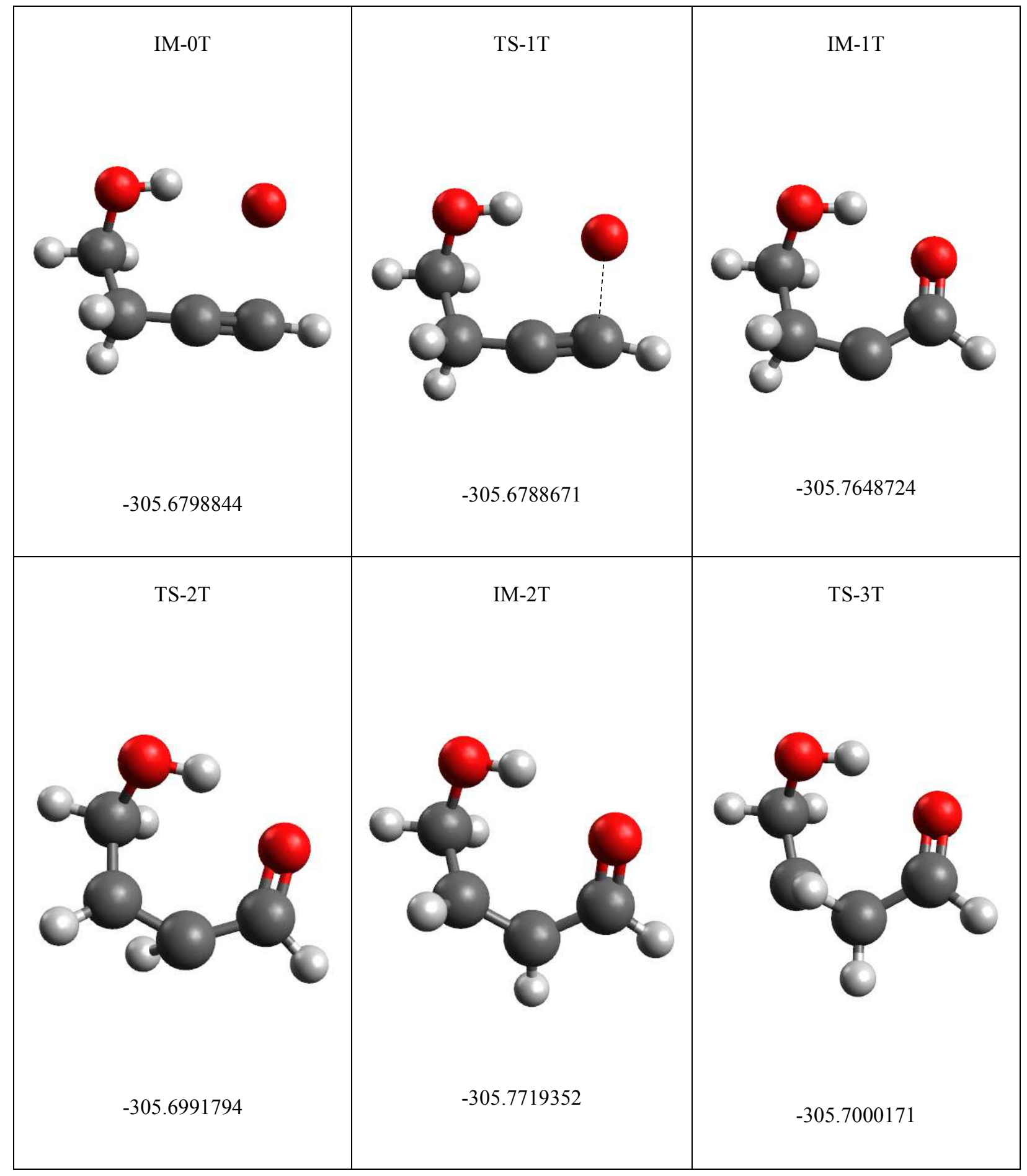




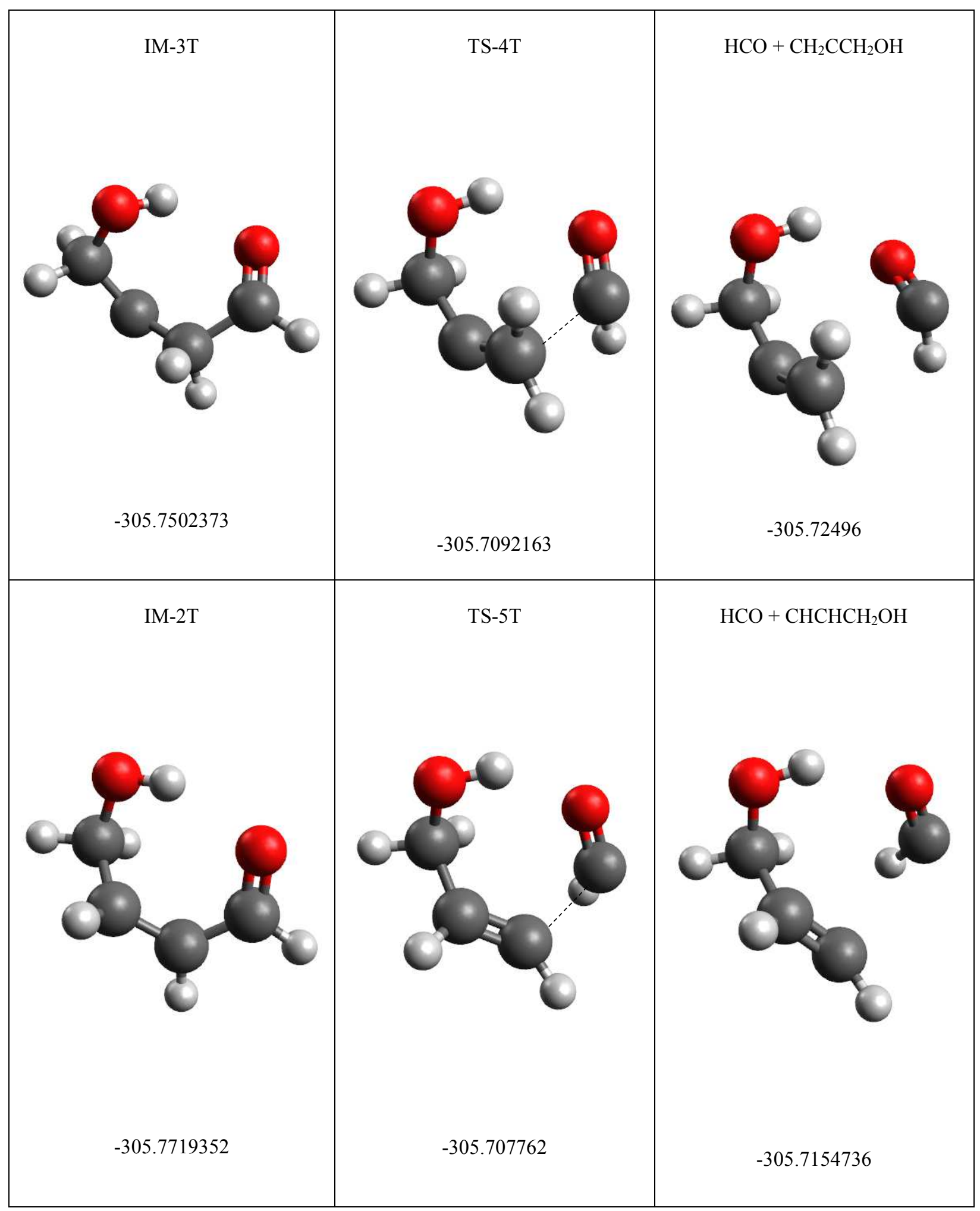




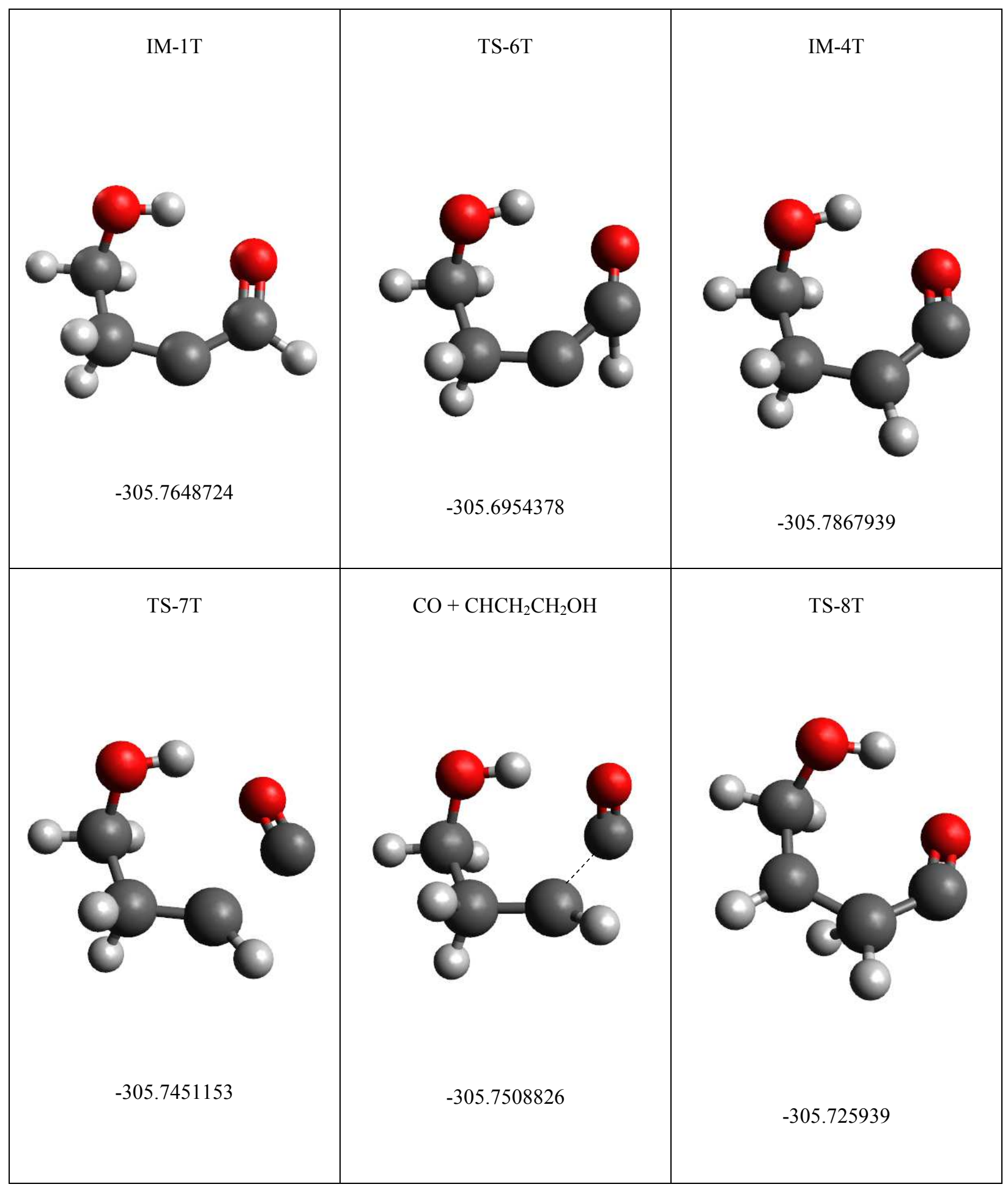




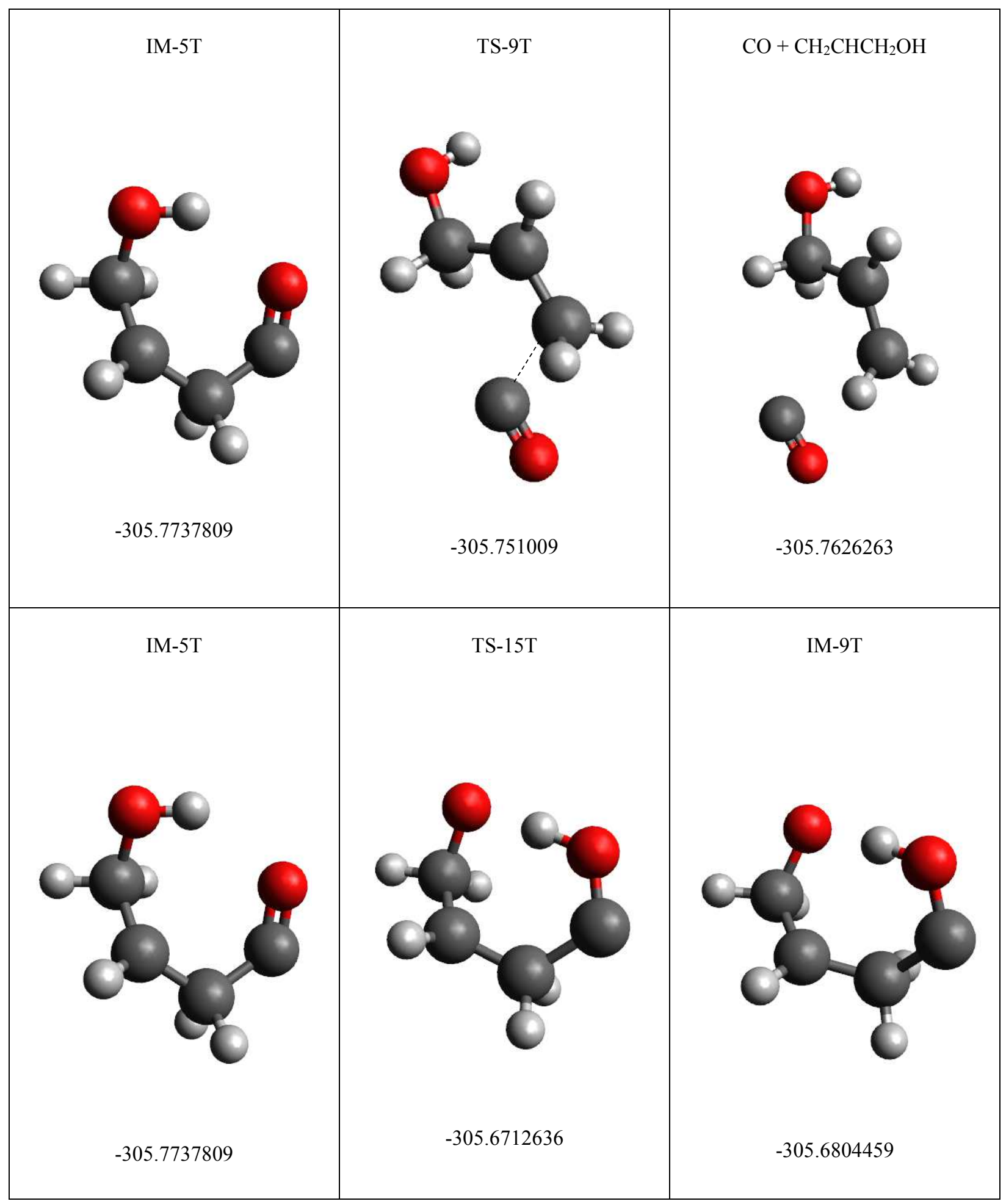




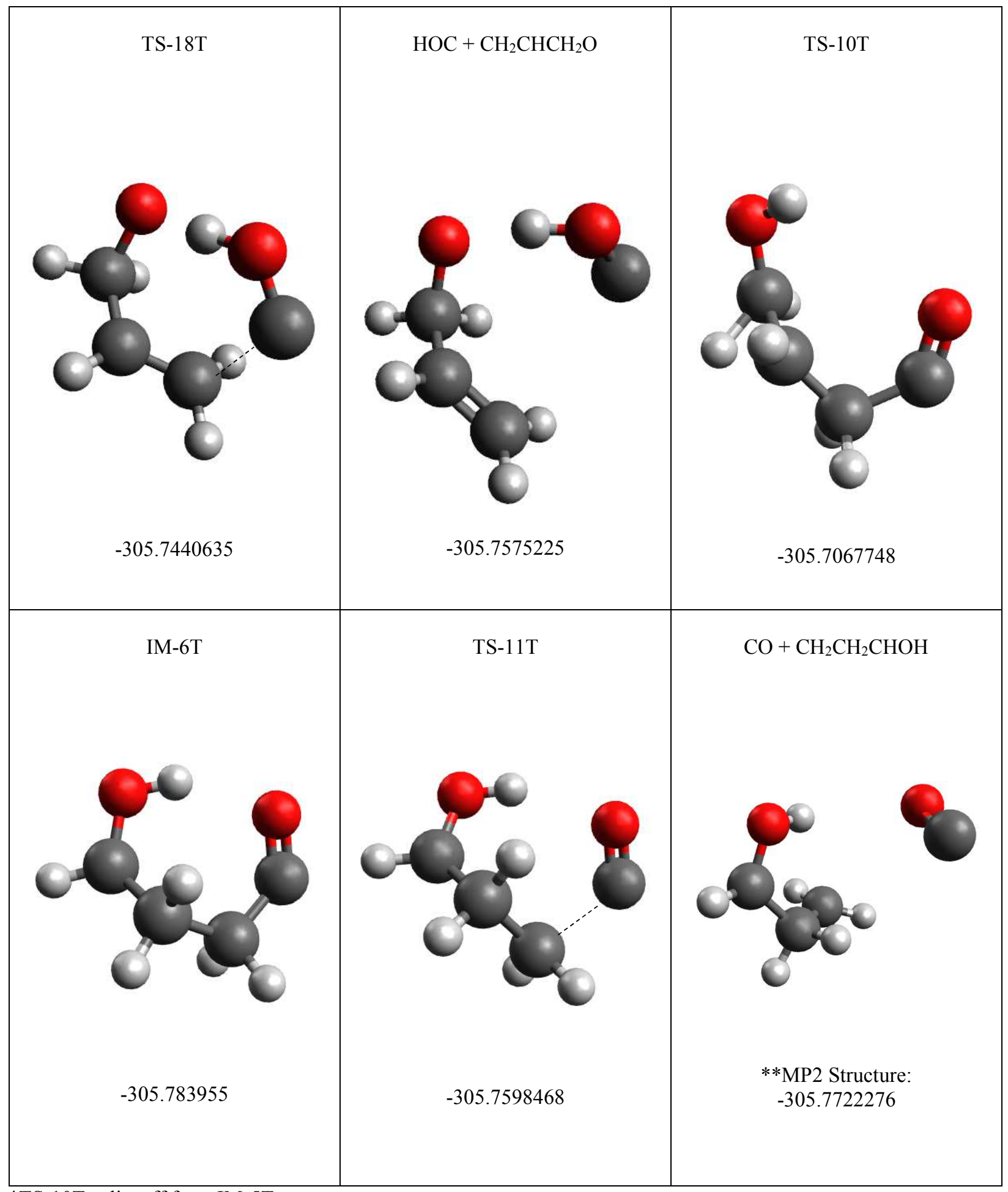

*TS-10T splits off from IM-5T

**M06-2x structure did not converge, and so MP2/6-311++G(d,p) was employed here. 


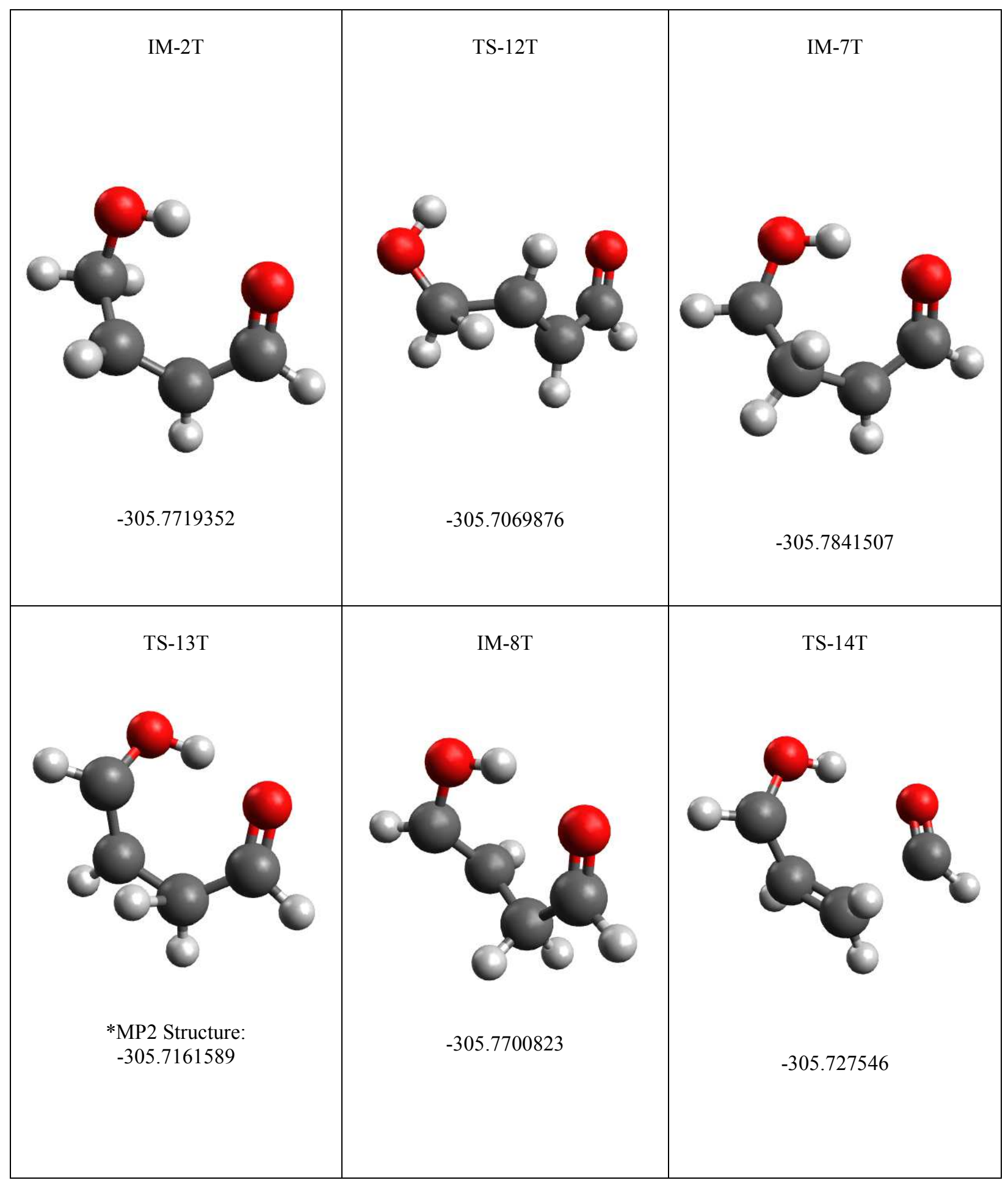




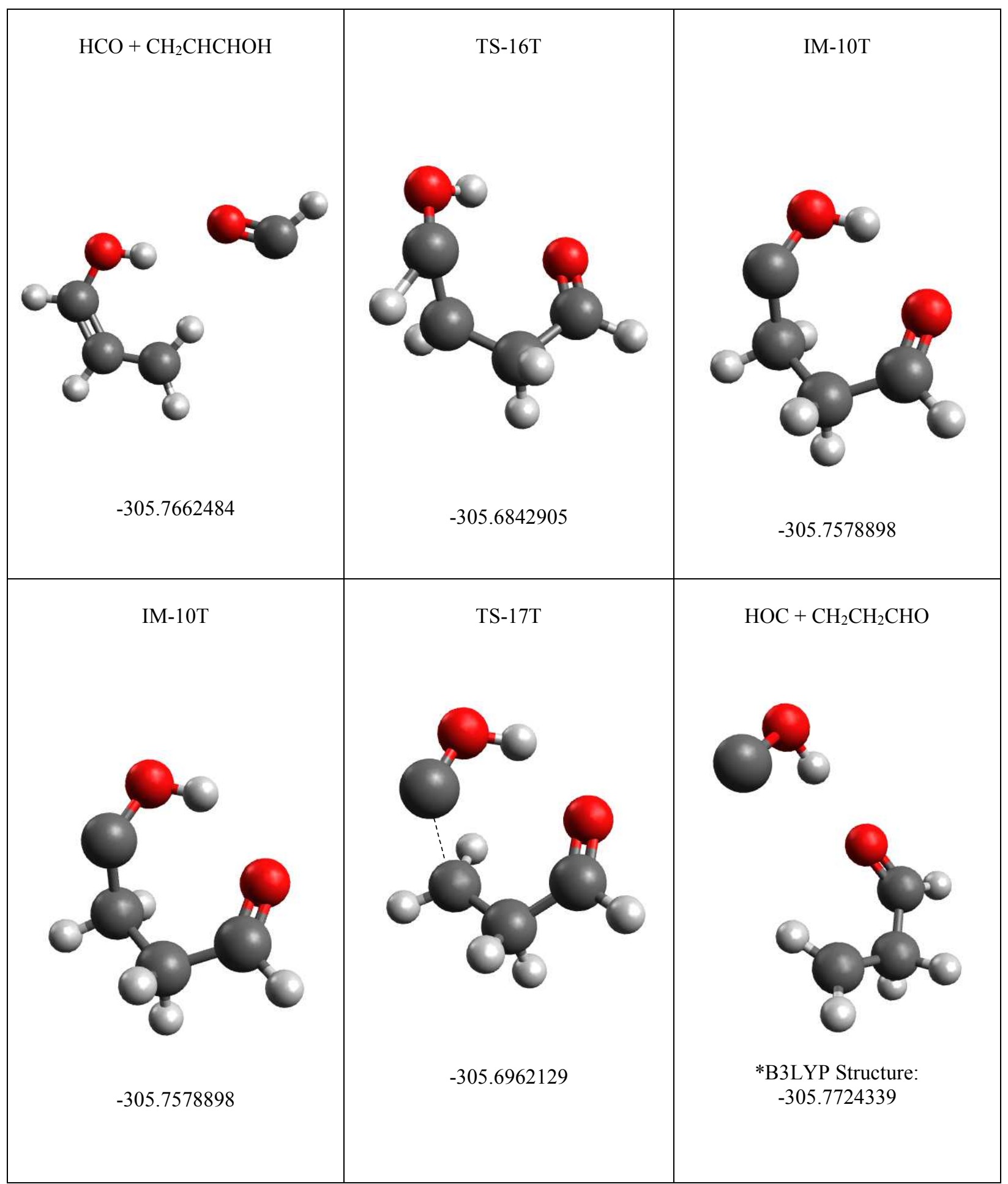

*HOC $+\mathrm{CH}_{2} \mathrm{CH}_{2} \mathrm{CHO}$ structure did not converge with M06-2x and so B3LYP, another DFT method, was employed. 
Subsequent Dissociation Pathway Structures: Doublet Surface, M06-2x/cc-pVTZ

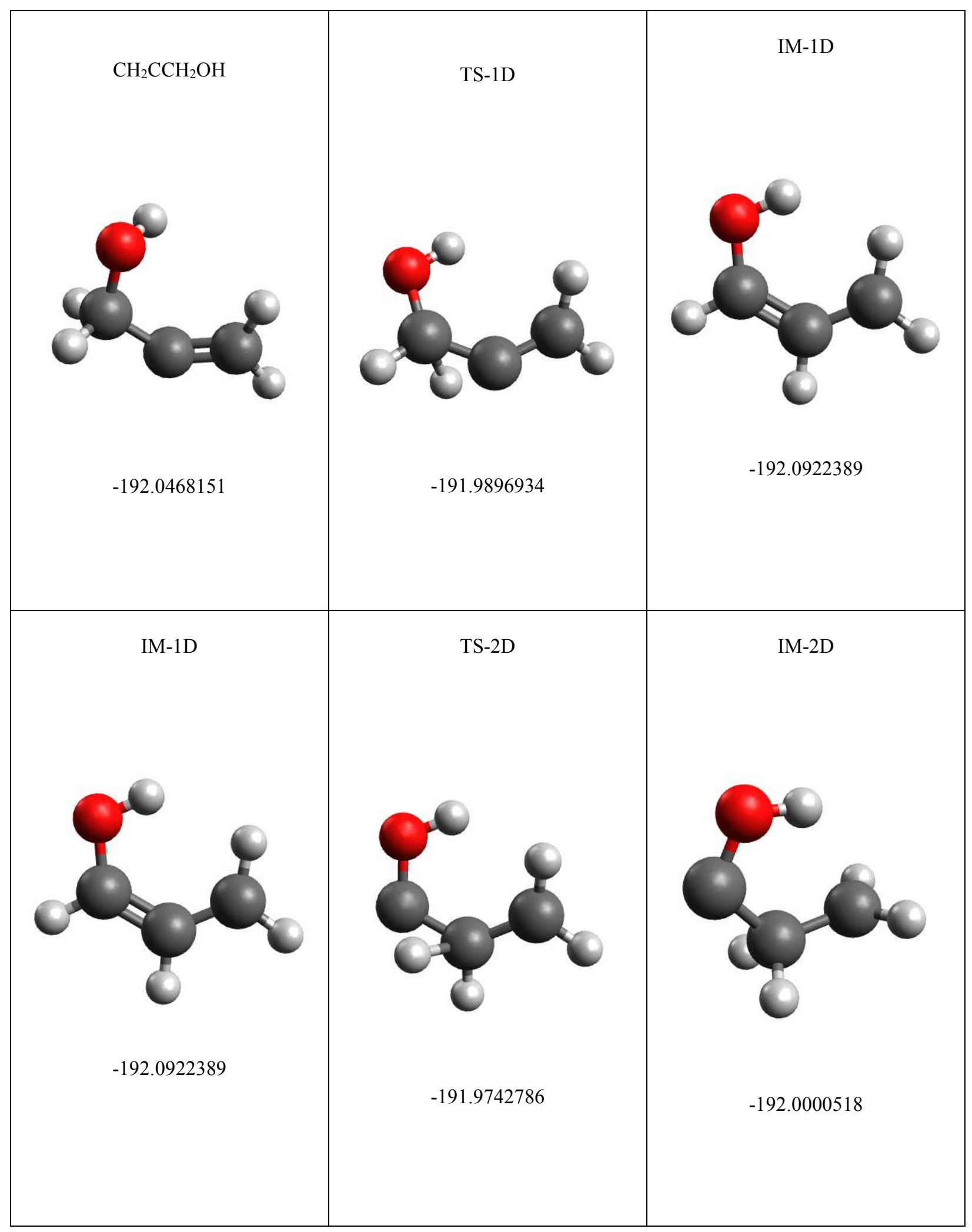




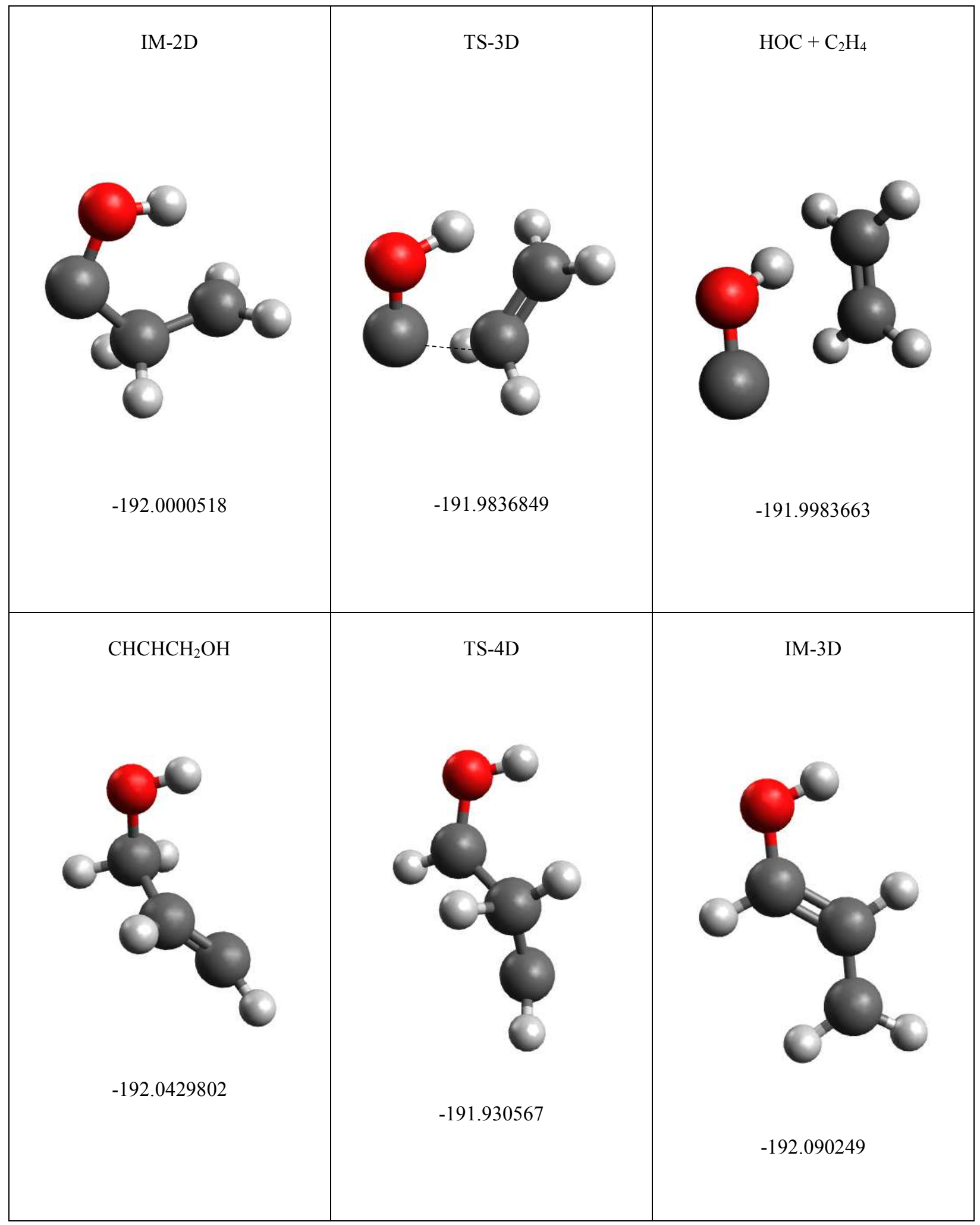




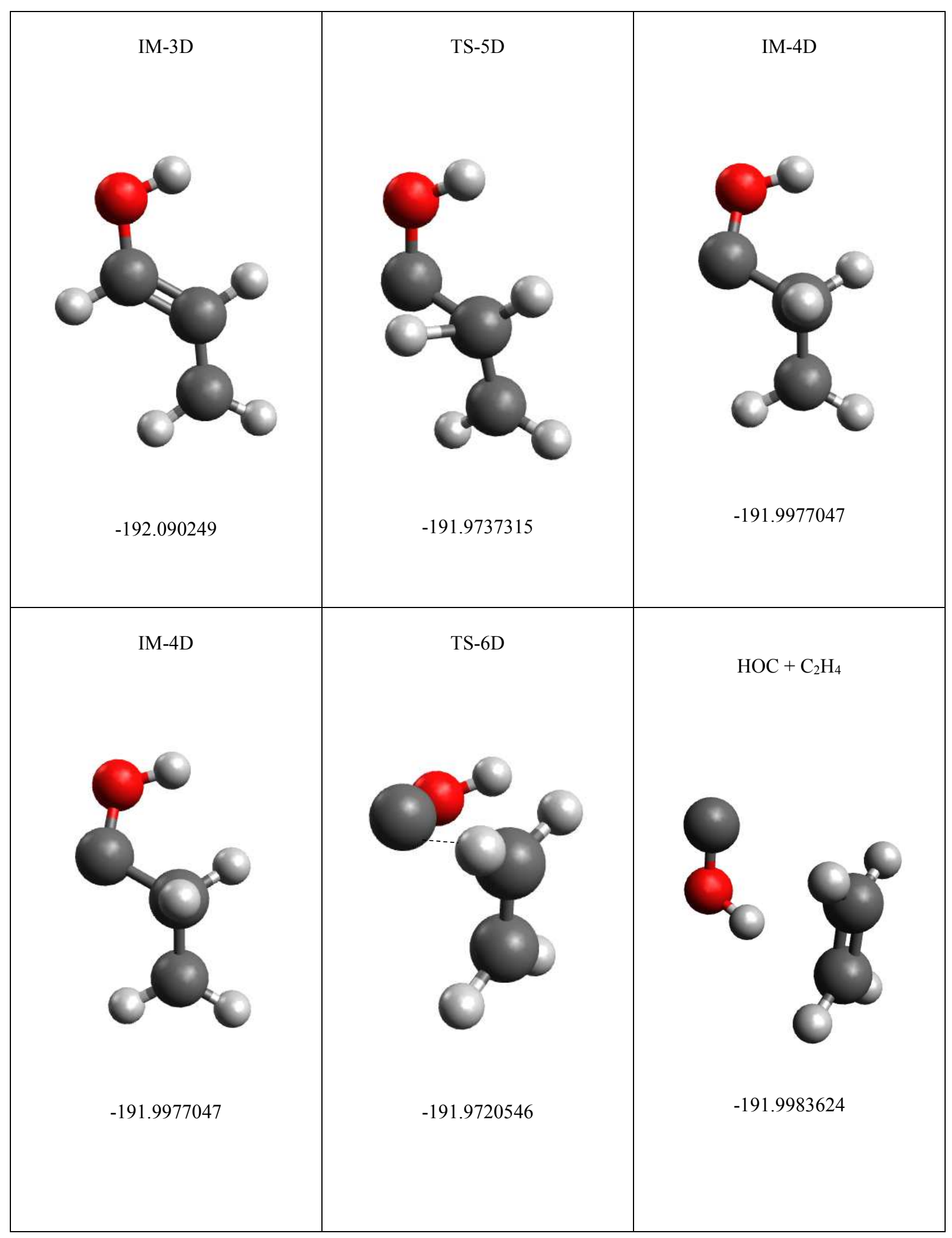




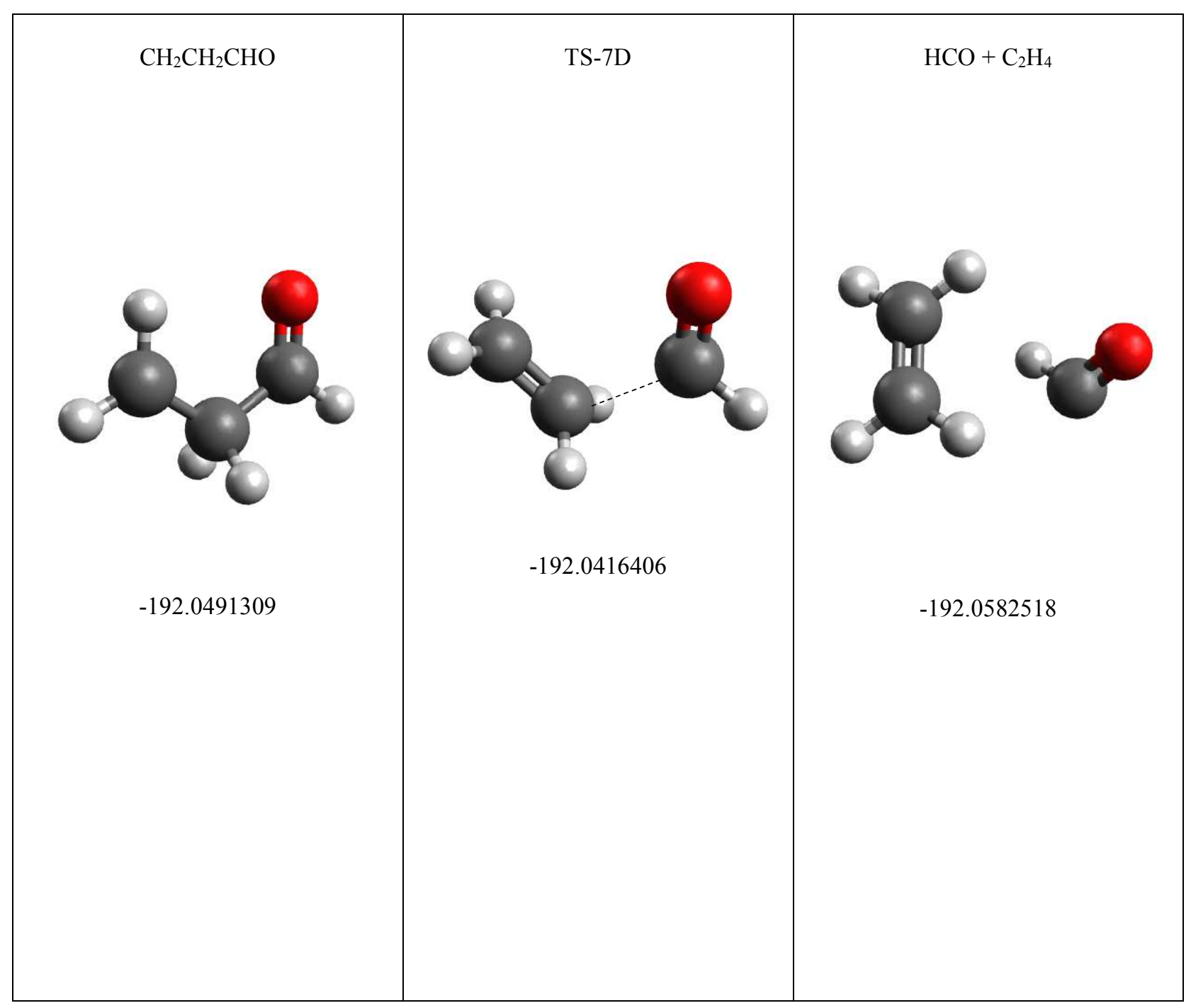


APPENDIX B: CARTESIAN COORDINATES FOR ALL OPTIMIZED STRUCTURES FOR $\mathrm{O}\left({ }^{3} \mathrm{P}\right)+$ PROPARGYL ALCOHOL AND O $\left({ }^{3} \mathrm{P}\right)+3$-BUTYN-1-OL AT THE M06-2X/CCPVTZ LEVEL

M06-2x/cc-pVTZ Cartesian Coordinates for the Triplet State Surface of $O\left({ }^{3} \mathrm{P}\right)+$ Propargyl Alcohol

\begin{tabular}{lccc}
\multicolumn{4}{c}{ Propargyl Alcohol } \\
C & -1.90771 & -0.21543 & -0.01289 \\
H & -2.91716 & -0.54622 & -0.02884 \\
C & -0.77209 & 0.16278 & -0.00509 \\
C & 0.63088 & 0.58771 & 0.03511 \\
H & 0.83074 & 1.26248 & -0.79642 \\
H & 0.80372 & 1.14552 & 0.96083 \\
O & 1.53624 & -0.48759 & -0.09609 \\
H & 1.28634 & -1.17141 & 0.53037
\end{tabular}

$\mathrm{IM}-1 \mathrm{~T}$ cis a

\begin{tabular}{lccc}
\multicolumn{4}{c}{ IM-1T cis a } \\
C & -1.50230 & -0.50759 & 0.00069 \\
H & -2.09972 & -1.42861 & 0.00038 \\
C & -0.09151 & -0.62572 & -0.02304 \\
C & 0.94898 & 0.41084 & -0.03028 \\
H & 0.82693 & 1.01416 & -0.94082 \\
H & 0.77015 & 1.08025 & 0.82260 \\
O & 2.21805 & -0.20156 & 0.03132 \\
H & 2.88872 & 0.48435 & 0.02596 \\
O & -2.03269 & 0.59964 & 0.01964
\end{tabular}

TS-1T

$\begin{array}{llll}\mathrm{C} & -1.37318 & -0.83850 & -0.10782 \\ \mathrm{H} & -2.39712 & -1.10119 & -0.22956 \\ \mathrm{C} & -0.15916 & -0.88809 & -0.01687 \\ \mathrm{C} & 1.25621 & -0.54661 & 0.14066 \\ \mathrm{H} & 1.55387 & -0.78051 & 1.16815 \\ \mathrm{H} & 1.86250 & -1.16090 & -0.52636 \\ \mathrm{O} & 1.49504 & 0.80493 & -0.17499 \\ \mathrm{H} & 0.69439 & 1.30076 & 0.03771 \\ \mathrm{O} & -1.50215 & 1.11771 & 0.10677\end{array}$

\begin{tabular}{lrrr}
\multicolumn{4}{c}{ IM-1T cis b } \\
C & -1.35867 & 0.39464 & 0.11736 \\
$\mathrm{H}$ & -2.24812 & 0.98135 & 0.37854 \\
$\mathrm{C}$ & -0.09764 & 1.03947 & 0.12634 \\
$\mathrm{C}$ & 1.23830 & 0.50370 & -0.20319 \\
$\mathrm{H}$ & 1.38753 & 0.60180 & -1.28879 \\
$\mathrm{H}$ & 2.01494 & 1.09949 & 0.27841 \\
$\mathrm{O}$ & 1.39754 & -0.82716 & 0.22911 \\
$\mathrm{H}$ & 0.59989 & -1.31104 & -0.02020 \\
$\mathrm{O}$ & -1.45331 & -0.79765 & -0.17799
\end{tabular}


IM-1T trans a

$\begin{array}{lrrr}\mathrm{C} & -1.17744 & -0.29718 & 0.00003 \\ \mathrm{H} & -0.81476 & -1.33989 & 0.00014 \\ \mathrm{C} & -0.14860 & 0.67771 & -0.00005 \\ \mathrm{C} & 1.31792 & 0.62283 & 0.00003 \\ \mathrm{H} & 1.69588 & 1.15435 & -0.88304 \\ \mathrm{H} & 1.69578 & 1.15418 & 0.88324 \\ \mathrm{O} & 1.72129 & -0.73555 & -0.00008 \\ \mathrm{H} & 2.68045 & -0.77545 & 0.00030 \\ \mathrm{O} & -2.37237 & -0.04112 & -0.00001\end{array}$

IM-1T trans c

$\begin{array}{lrrr}\mathrm{C} & 1.37109 & 0.18442 & 0.25945 \\ \mathrm{H} & 1.32239 & 0.84279 & 1.15032 \\ \mathrm{C} & 0.12579 & -0.00395 & -0.39843 \\ \mathrm{C} & -1.22597 & 0.51179 & -0.13930 \\ \mathrm{H} & -1.17237 & 1.23241 & 0.69107 \\ \mathrm{H} & -1.57535 & 1.05539 & -1.02564 \\ \mathrm{O} & -2.08081 & -0.57082 & 0.17002 \\ \mathrm{H} & -2.98404 & -0.24791 & 0.20787 \\ \mathrm{O} & 2.42880 & -0.30872 & -0.08927\end{array}$

IM-1T trans b

$\begin{array}{lrrr}\mathrm{C} & -1.36112 & 0.15316 & 0.27972 \\ \mathrm{H} & -1.29560 & 0.69664 & 1.24416 \\ \mathrm{C} & -0.12706 & 0.04897 & -0.41795 \\ \mathrm{C} & 1.23026 & 0.52469 & -0.11421 \\ \mathrm{H} & 1.60060 & 1.11193 & -0.96302 \\ \mathrm{H} & 1.17934 & 1.19622 & 0.75632 \\ \mathrm{O} & 2.06158 & -0.59021 & 0.14219 \\ \mathrm{H} & 2.97017 & -0.28735 & 0.20832 \\ \mathrm{O} & -2.42495 & -0.29458 & -0.10858\end{array}$

TS-2T cis a

$\begin{array}{llll}\mathrm{C} & -1.50086 & 0.45887 & -0.08185 \\ \mathrm{H} & -2.13547 & 1.35289 & -0.12765 \\ \mathrm{C} & -0.09405 & 0.66371 & 0.08144 \\ \mathrm{C} & 0.97694 & -0.28473 & 0.23059 \\ \mathrm{H} & 0.46543 & 0.57137 & 1.20632 \\ \mathrm{H} & 0.72265 & -1.31952 & 0.43752 \\ \mathrm{O} & 2.19292 & 0.03341 & -0.28601 \\ \mathrm{H} & 2.87057 & -0.50700 & 0.12900 \\ \mathrm{O} & -1.96984 & -0.67401 & -0.09228\end{array}$


TS-2T cis b

$\begin{array}{lrrr}\mathrm{C} & 1.44639 & 0.34150 & 0.02210 \\ \mathrm{H} & 2.28909 & 1.02354 & 0.19367 \\ \mathrm{C} & 0.15313 & 0.93052 & -0.02650 \\ \mathrm{C} & -1.21630 & 0.51747 & -0.11576 \\ \mathrm{H} & -0.63843 & 1.23883 & 0.91882 \\ \mathrm{H} & -1.91547 & 1.18330 & -0.61052 \\ \mathrm{O} & -1.54411 & -0.78887 & 0.05218 \\ \mathrm{H} & -2.47940 & -0.86288 & 0.26388 \\ \mathrm{O} & 1.59972 & -0.87609 & -0.05779\end{array}$

TS-2T trans b

$\begin{array}{llll}\mathrm{C} & -1.21427 & -0.32151 & 0.08366 \\ \mathrm{H} & -0.88004 & -1.36463 & 0.24043 \\ \mathrm{C} & -0.13511 & 0.60597 & 0.03360 \\ \mathrm{C} & 1.30019 & 0.56742 & 0.07879 \\ \mathrm{H} & 0.51644 & 1.04507 & -0.96015 \\ \mathrm{H} & 1.83556 & 1.38791 & 0.54398 \\ \mathrm{O} & 1.89916 & -0.65514 & -0.02712 \\ \mathrm{H} & 2.80460 & -0.55015 & -0.33360 \\ \mathrm{O} & -2.39684 & -0.04854 & -0.05625\end{array}$

TS-2T trans a

$\begin{array}{lrrr}\mathrm{C} & 1.39978 & 0.26451 & -0.19958 \\ \mathrm{H} & 1.36669 & 1.23903 & -0.72922 \\ \mathrm{C} & 0.10893 & -0.19345 & 0.22695 \\ \mathrm{C} & -1.21105 & 0.38588 & 0.19925 \\ \mathrm{H} & -0.52822 & -0.03257 & 1.31730 \\ \mathrm{H} & -1.29286 & 1.47199 & 0.17032 \\ \mathrm{O} & -2.23856 & -0.37213 & -0.27904 \\ \mathrm{H} & -3.07141 & -0.04297 & 0.07045 \\ \mathrm{O} & 2.45604 & -0.30002 & 0.00547\end{array}$

IM-2T cis a

$\begin{array}{llll}\mathrm{C} & -1.38868 & 0.25105 & -0.17666 \\ \mathrm{H} & -2.22879 & 0.83983 & -0.58102 \\ \mathrm{C} & -0.14188 & 0.95313 & 0.00379 \\ \mathrm{C} & 1.04698 & 0.27071 & 0.50973 \\ \mathrm{H} & -0.10848 & 2.00602 & -0.25506 \\ \mathrm{H} & 1.03989 & -0.13879 & 1.51256 \\ \mathrm{O} & 1.76242 & -0.39507 & -0.44109 \\ \mathrm{H} & 2.38539 & -0.99138 & -0.01805 \\ \mathrm{O} & -1.53574 & -0.92556 & 0.10615\end{array}$


IM- $2 \mathrm{~T}$ cis b

IM-2T trans a

$\begin{array}{llllllll}\mathrm{C} & 1.38849 & 0.25087 & -0.17671 & \mathrm{C} & -1.18739 & -0.27787 & 0.24099 \\ \mathrm{H} & 2.22871 & 0.83919 & -0.58151 & \mathrm{H} & -0.90893 & -1.08482 & 0.94083 \\ \mathrm{C} & 0.14188 & 0.95339 & 0.00356 & \mathrm{C} & -0.12069 & 0.60156 & -0.16561 \\ \mathrm{C} & -1.04722 & 0.27144 & 0.50954 & \mathrm{C} & 1.24733 & 0.45386 & 0.31639 \\ \mathrm{H} & 0.10877 & 2.00618 & -0.25570 & \mathrm{H} & -0.38882 & 1.38589 & -0.86664 \\ \mathrm{O} & -1.76150 & -0.39581 & -0.44110 & \mathrm{H} & 1.51441 & 0.70165 & 1.33657 \\ \mathrm{H} & -1.04016 & -0.13761 & 1.51259 & \mathrm{O} & 1.99650 & -0.48419 & -0.33245 \\ \mathrm{O} & 1.53521 & -0.92564 & 0.10662 & \mathrm{H} & 2.85693 & -0.55994 & 0.08863 \\ \mathrm{H} & -2.38594 & -0.99034 & -0.01781 & \mathrm{O} & -2.33514 & -0.15432 & -0.14880\end{array}$

IM-2T trans b

TS-3T isomer a

$\begin{array}{llllllll}\mathrm{C} & 1.18737 & -0.27786 & 0.24099 & \mathrm{C} & -1.46481 & 0.40483 & -0.14617 \\ \mathrm{H} & 0.90892 & -1.08478 & 0.94088 & \mathrm{H} & -0.89401 & 1.41361 & 0.45880 \\ \mathrm{C} & 0.12068 & 0.60160 & -0.16559 & \mathrm{C} & -0.09693 & 0.48594 & -0.28601 \\ \mathrm{C} & -1.24734 & 0.45387 & 0.31641 & \mathrm{C} & 1.03740 & -0.46583 & -0.17330 \\ \mathrm{H} & 0.38881 & 1.38592 & -0.86662 & \mathrm{H} & 0.74392 & -1.24320 & 0.54506 \\ \mathrm{O} & -1.99646 & -0.48419 & -0.33248 & \mathrm{H} & 1.16428 & -0.94683 & -1.15145 \\ \mathrm{H} & -1.51430 & 0.70139 & 1.33668 & \mathrm{O} & 2.19462 & 0.22373 & 0.23001 \\ \mathrm{O} & 2.33511 & -0.15434 & -0.14882 & \mathrm{H} & 2.92607 & -0.39658 & 0.26550 \\ \mathrm{H} & -2.85691 & -0.55993 & 0.08858 & \mathrm{O} & -2.29390 & -0.39581 & 0.20937\end{array}$


TS-3T isomer b

$\begin{array}{lrrr}\mathrm{C} & -1.29795 & 0.44752 & -0.03189 \\ \mathrm{H} & -1.09167 & 1.25182 & 0.97108 \\ \mathrm{C} & -0.04551 & 1.02172 & -0.00059 \\ \mathrm{C} & 1.33180 & 0.44062 & -0.12819 \\ \mathrm{H} & 2.02018 & 0.92880 & 0.55807 \\ \mathrm{O} & 1.34051 & -0.93189 & 0.17427 \\ \mathrm{H} & 1.67210 & 0.64559 & -1.15144 \\ \mathrm{O} & -1.73227 & -0.68337 & -0.06703 \\ \mathrm{H} & 0.60335 & -1.36333 & -0.27161\end{array}$

IM-3T cis b

$\begin{array}{llll}\mathrm{C} & 1.33249 & 0.44898 & -0.08385 \\ \mathrm{C} & 0.05221 & 1.09686 & -0.07674 \\ \mathrm{H} & 0.02896 & 2.17449 & -0.14756 \\ \mathrm{C} & -1.23266 & 0.36725 & 0.13215 \\ \mathrm{O} & -1.20682 & -1.00115 & -0.14736 \\ \mathrm{H} & -1.54708 & 0.56367 & 1.17202 \\ \mathrm{H} & -2.00041 & 0.81837 & -0.50272 \\ \mathrm{O} & 1.57739 & -0.70736 & 0.08766 \\ \mathrm{H} & -0.35824 & -1.36689 & 0.12641\end{array}$

IM-3T cis a

$\begin{array}{llll}\mathrm{C} & -1.52452 & 0.51327 & 0.00006 \\ \mathrm{C} & -0.10274 & 0.72741 & -0.00019 \\ \mathrm{H} & 0.29887 & 1.72927 & -0.00003 \\ \mathrm{C} & 0.82928 & -0.42730 & -0.00017 \\ \mathrm{H} & 0.61461 & -1.05035 & 0.87904 \\ \mathrm{H} & 0.61503 & -1.05013 & -0.87963 \\ \mathrm{O} & 2.15131 & 0.05731 & 0.00023 \\ \mathrm{H} & 2.75955 & -0.68448 & -0.00017 \\ \mathrm{O} & -2.08883 & -0.53538 & 0.00009\end{array}$

IM-3T trans a

$\begin{array}{lrrr}\mathrm{C} & 1.42026 & 0.33837 & -0.00023 \\ \mathrm{C} & 0.14010 & -0.30871 & 0.00007 \\ \mathrm{H} & 0.06912 & -1.39177 & 0.00059 \\ \mathrm{C} & -1.10143 & 0.49544 & 0.00011 \\ \mathrm{H} & -1.09759 & 1.15413 & 0.88020 \\ \mathrm{H} & -1.09737 & 1.15464 & -0.87960 \\ \mathrm{O} & -2.20542 & -0.37743 & -0.00027 \\ \mathrm{H} & -3.01512 & 0.13665 & 0.00081 \\ \mathrm{O} & 2.50385 & -0.14811 & 0.00006\end{array}$


IM-3T trans b

$\begin{array}{lrrrrrrr}\mathrm{C} & 1.11511 & -0.33586 & -0.00004 & \mathrm{C} & -1.35964 & -0.33297 & -0.39707 \\ \mathrm{C} & 0.16609 & 0.74334 & 0.00002 & \mathrm{H} & -0.79942 & -1.29055 & -0.50749 \\ \mathrm{H} & 0.54077 & 1.76266 & 0.00010 & \mathrm{C} & 0.23315 & 1.07562 & -0.14505 \\ \mathrm{C} & -1.29888 & 0.54177 & -0.00002 & \mathrm{C} & 1.10764 & 0.27624 & 0.45788 \\ \mathrm{O} & -1.60678 & -0.82757 & 0.00002 & \mathrm{H} & 0.16360 & 1.61636 & -1.07072 \\ \mathrm{H} & -1.71706 & 1.05408 & 0.87952 & \mathrm{H} & 1.10180 & 0.15099 & 1.53591 \\ \mathrm{H} & -1.71701 & 1.05401 & -0.87964 & \mathrm{O} & 1.97408 & -0.50963 & -0.24449 \\ \mathrm{O} & 2.30171 & -0.25155 & 0.00001 & \mathrm{H} & 2.41529 & -1.11951 & 0.35216 \\ \mathrm{H} & -2.56007 & -0.93324 & 0.00002 & \mathrm{O} & -2.32010 & -0.17419 & 0.26893\end{array}$

TS-4T

\section{TS-5T out}

$\begin{array}{llll}\mathrm{C} & -1.74903 & 0.31732 & -0.33890 \\ \mathrm{C} & 0.27315 & 1.22083 & -0.08705 \\ \mathrm{H} & 0.45164 & 2.10190 & -0.68081 \\ \mathrm{C} & 1.16658 & 0.21175 & 0.49239 \\ \mathrm{H} & 2.09973 & 0.68965 & 0.81961 \\ \mathrm{H} & 0.68800 & -0.22550 & 1.37638 \\ \mathrm{O} & 1.42988 & -0.79189 & -0.48174 \\ \mathrm{H} & 1.82865 & -1.54715 & -0.04285 \\ \mathrm{O} & -1.83141 & -0.64789 & 0.24788\end{array}$


TS-5T in

$\begin{array}{llll}\mathrm{C} & -1.55846 & -0.25750 & 0.48406 \\ \mathrm{C} & 0.16930 & 0.96005 & -0.22257 \\ \mathrm{H} & -0.03773 & 1.54574 & -1.10495 \\ \mathrm{C} & 1.40977 & 0.41020 & 0.33092 \\ \mathrm{H} & 2.22717 & 1.13077 & 0.19446 \\ \mathrm{H} & 1.27623 & 0.25175 & 1.40681 \\ \mathrm{O} & 1.70692 & -0.81768 & -0.32517 \\ \mathrm{H} & 2.46054 & -1.22144 & 0.11237 \\ \mathrm{O} & -2.46316 & -0.23024 & -0.19522\end{array}$

TS-6T cis a

$\begin{array}{lrrr}\mathrm{C} & -1.45907 & 0.43697 & -0.10812 \\ \mathrm{C} & -0.04226 & 0.69502 & -0.06557 \\ \mathrm{H} & -1.12651 & 1.04115 & 0.96230 \\ \mathrm{C} & 0.88471 & -0.33211 & 0.02664 \\ \mathrm{H} & 0.28819 & 1.71773 & -0.17539 \\ \mathrm{H} & 0.57424 & -1.36583 & 0.09027 \\ \mathrm{O} & 2.19968 & -0.04110 & 0.00565 \\ \mathrm{H} & 2.71992 & -0.84719 & -0.04266 \\ \mathrm{O} & -2.04420 & -0.62703 & 0.00032\end{array}$

$\mathrm{CH}_{2} \mathrm{OHCH}+\mathrm{CO}$

$\begin{array}{lrrr}\mathrm{C} & 1.31417 & -0.22309 & -0.14134 \\ \mathrm{H} & 1.89369 & -0.85557 & 0.51152 \\ \mathrm{C} & 0.05692 & 0.50493 & 0.03198 \\ \mathrm{H} & 0.06859 & 1.03441 & 0.99628 \\ \mathrm{H} & -0.03040 & 1.26404 & -0.75444 \\ \mathrm{O} & -1.03832 & -0.40400 & -0.02096 \\ \mathrm{H} & -1.85186 & 0.09805 & 0.07046 \\ \mathrm{C} & 0.00000 & 0.00000 & -0.64106 \\ \mathrm{O} & 0.00000 & 0.00000 & 0.48079\end{array}$

TS-6T cis b

$\begin{array}{llll}\mathrm{C} & -1.25243 & 0.40756 & -0.07351 \\ \mathrm{C} & 0.01862 & 1.09151 & -0.05043 \\ \mathrm{H} & -1.01627 & 0.99931 & 1.04390 \\ \mathrm{C} & 1.21458 & 0.37944 & -0.02498 \\ \mathrm{H} & 0.01995 & 2.16732 & -0.12186 \\ \mathrm{O} & 1.26586 & -0.94496 & 0.04226 \\ \mathrm{H} & 2.17769 & 0.86361 & -0.09716 \\ \mathrm{O} & -1.44345 & -0.80518 & -0.04016 \\ \mathrm{H} & 0.35474 & -1.30021 & 0.05192\end{array}$


TS- $6 \mathrm{~T}$ trans a

$\begin{array}{lrrr}\mathrm{C} & -1.35561 & 0.34167 & 0.03375 \\ \mathrm{C} & -0.06015 & -0.32139 & -0.00241 \\ \mathrm{H} & -0.84314 & 0.23045 & 1.19030 \\ \mathrm{C} & 1.11526 & 0.38894 & -0.07207 \\ \mathrm{H} & -0.02917 & -1.40899 & -0.00881 \\ \mathrm{H} & 1.12259 & 1.46943 & -0.13676 \\ \mathrm{O} & 2.29097 & -0.26447 & 0.01909 \\ \mathrm{H} & 3.02125 & 0.33686 & -0.14473 \\ \mathrm{O} & -2.47454 & -0.12091 & -0.10104\end{array}$

IM-4T cis a

$\begin{array}{lrrr}\mathrm{C} & 1.52920 & 0.43374 & -0.02488 \\ \mathrm{C} & 0.06328 & 0.79953 & 0.03179 \\ \mathrm{H} & -0.13518 & 1.51510 & -0.76998 \\ \mathrm{H} & -0.06963 & 1.36483 & 0.96487 \\ \mathrm{C} & -0.82780 & -0.38620 & -0.05412 \\ \mathrm{H} & -0.50558 & -1.34156 & 0.33845 \\ \mathrm{O} & 2.00428 & -0.64038 & -0.01391 \\ \mathrm{O} & -2.15433 & -0.07693 & -0.01601 \\ \mathrm{H} & -2.67732 & -0.88232 & -0.01077\end{array}$

TS-6T trans b

$\begin{array}{llll}\mathrm{C} & 1.02289 & -0.27406 & 0.04975 \\ \mathrm{C} & 0.03627 & 0.79941 & -0.01013 \\ \mathrm{H} & 0.59178 & 0.07335 & 1.20523 \\ \mathrm{C} & -1.31106 & 0.50521 & -0.04529 \\ \mathrm{H} & 0.37420 & 1.82819 & -0.07465 \\ \mathrm{O} & -1.77509 & -0.74398 & -0.01686 \\ \mathrm{H} & -2.07748 & 1.26379 & -0.07386 \\ \mathrm{O} & 2.23029 & -0.25566 & -0.10164 \\ \mathrm{H} & -1.01872 & -1.35151 & -0.07463\end{array}$

IM-4T cis b

$\begin{array}{llll}\mathrm{C} & 1.38181 & 0.22015 & 0.23544 \\ \mathrm{C} & 0.11424 & 1.05006 & 0.14223 \\ \mathrm{H} & 0.37077 & 1.92195 & -0.46078 \\ \mathrm{H} & -0.09161 & 1.42369 & 1.15291 \\ \mathrm{C} & -1.04912 & 0.32117 & -0.43850 \\ \mathrm{O} & -1.45332 & -0.73609 & 0.31593 \\ \mathrm{O} & 1.58699 & -0.83951 & -0.22307 \\ \mathrm{H} & -1.81098 & 0.86494 & -0.98586 \\ \mathrm{H} & -2.21921 & -1.15404 & -0.08415\end{array}$


IM-4T trans a

$\begin{array}{lrrrrrrr}\mathrm{C} & -1.38207 & 0.36983 & 0.04102 & \mathrm{C} & 1.14780 & -0.10661 & -0.39411 \\ \mathrm{C} & -0.09103 & -0.42306 & 0.10863 & \mathrm{C} & 0.09711 & 0.81619 & 0.21101 \\ \mathrm{H} & -0.12785 & -1.20589 & -0.65559 & \mathrm{H} & 0.35216 & 1.81700 & -0.14239 \\ \mathrm{H} & -0.10378 & -0.93305 & 1.08423 & \mathrm{H} & 0.22119 & 0.79529 & 1.30338 \\ \mathrm{C} & 1.09230 & 0.45226 & -0.05709 & \mathrm{C} & -1.27103 & 0.45829 & -0.23013 \\ \mathrm{H} & 1.07482 & 1.46614 & 0.32368 & \mathrm{O} & -1.64720 & -0.79585 & 0.14294 \\ \mathrm{O} & -2.47117 & -0.04399 & -0.09256 & \mathrm{O} & 2.16790 & -0.43583 & 0.08194 \\ \mathrm{O} & 2.27566 & -0.22111 & -0.05643 & \mathrm{H} & -2.04220 & 1.21210 & -0.32182 \\ \mathrm{H} & 3.00564 & 0.39942 & -0.11578 & \mathrm{H} & -2.54005 & -0.97813 & -0.15877\end{array}$

TS-7T isomer a

$\begin{array}{lrrr}\mathrm{C} & 1.67406 & -0.53124 & 0.03320 \\ \mathrm{C} & -0.12130 & 0.63754 & 0.02219 \\ \mathrm{H} & 0.11942 & 1.25364 & -0.83837 \\ \mathrm{H} & 0.02373 & 1.13183 & 0.98156 \\ \mathrm{C} & -1.16300 & -0.37693 & -0.10298 \\ \mathrm{H} & -0.97594 & -1.40189 & 0.19220 \\ \mathrm{O} & 2.63694 & 0.06870 & -0.02194 \\ \mathrm{O} & -2.44295 & 0.09206 & 0.01537 \\ \mathrm{H} & -3.05782 & -0.64590 & 0.00265\end{array}$

TS-7T isomer b

$\begin{array}{lrrr}\mathrm{C} & 1.35066 & -0.00792 & -0.54352 \\ \mathrm{C} & -0.29761 & 1.20242 & 0.06575 \\ \mathrm{H} & -0.19858 & 1.98057 & -0.68073 \\ \mathrm{H} & 0.06978 & 1.47741 & 1.05356 \\ \mathrm{C} & -1.44168 & 0.29546 & -0.03852 \\ \mathrm{O} & -1.15579 & -1.04289 & -0.00842 \\ \mathrm{O} & 2.00996 & -0.38713 & 0.30051 \\ \mathrm{H} & -2.39933 & 0.58843 & 0.38436 \\ \mathrm{H} & -1.97343 & -1.54610 & 0.00381\end{array}$




\begin{tabular}{lrrrrrrr}
\multicolumn{3}{c}{$\mathrm{CHOHCH}_{2}+\mathrm{CO}$} & & & \multicolumn{3}{c}{$\mathrm{HCO}$} \\
$\mathrm{C}$ & -1.27409 & -0.13819 & 0.01359 & $\mathrm{C}$ & 0.06197 & 0.58133 & 0.00000 \\
$\mathrm{H}$ & -1.84664 & -0.38202 & -0.87158 & $\mathrm{H}$ & -0.86759 & 1.20850 & 0.00000 \\
$\mathrm{H}$ & -1.67795 & -0.46029 & 0.96770 & $\mathrm{O}$ & 0.06197 & -0.58706 & 0.00000 \\
$\mathrm{C}$ & 0.03246 & 0.48565 & -0.08338 & & & & \\
$\mathrm{H}$ & 0.19311 & 1.50434 & 0.25545 & & & & \\
$\mathrm{O}$ & 1.10781 & -0.36241 & 0.00676 & & & & \\
$\mathrm{H}$ & 1.91876 & 0.15250 & 0.01313 & & & & \\
$\mathrm{C}$ & 0.00000 & 0.00000 & -0.64106 & & & & \\
$\mathrm{O}$ & 0.00000 & 0.00000 & 0.48079 & & & &
\end{tabular}

\section{M06-2x/cc-pVTZ Cartesian Coordinates for the Singlet State Surface of $O\left({ }^{3} P\right)+$ Propargyl} Alcohol

\begin{tabular}{lrrrrrrr} 
& \multicolumn{2}{c}{ IM-1S noncyclic } & & \multicolumn{3}{c}{ IM-1S cyclic } \\
C & -1.19396 & 0.46296 & -0.18245 & C & 1.08189 & -0.82586 & -0.09224 \\
C & 0.12305 & 1.06398 & -0.13546 & C & 0.15557 & 0.01112 & 0.65629 \\
H & -1.63696 & 0.64287 & -1.17150 & H & 1.84757 & -1.39050 & 0.42999 \\
H & -1.76583 & 1.13796 & 0.48051 & O & 1.43761 & 0.60338 & -0.24448 \\
O & -1.37879 & -0.86023 & 0.24905 & H & 0.83240 & -1.22662 & -1.07102 \\
C & 1.19081 & 0.27806 & 0.38247 & C & -1.05549 & 0.42985 & -0.01886 \\
H & 1.68008 & 0.53217 & 1.33592 & H & -1.06764 & 1.47849 & -0.37484 \\
O & 1.58880 & -0.60768 & -0.36157 & O & -2.03603 & -0.27842 & -0.09101 \\
H & -0.67682 & -1.39966 & -0.13215 & H & 2.08317 & 0.84822 & 0.42866
\end{tabular}


TS-2S a

$\begin{array}{llll}\mathrm{C} & -1.41891 & 0.39289 & 0.05914 \\ \mathrm{H} & -1.77250 & 1.35184 & 0.48899 \\ \mathrm{C} & -0.09395 & 0.40122 & -0.48699 \\ \mathrm{C} & 0.96898 & -0.00605 & 0.34569 \\ \mathrm{H} & 0.86705 & 1.02629 & 0.83244 \\ \mathrm{H} & 0.76213 & -0.73327 & 1.14007 \\ \mathrm{O} & 2.21232 & -0.16673 & -0.25211 \\ \mathrm{H} & 2.85076 & -0.43587 & 0.41264 \\ \mathrm{O} & -2.14284 & -0.57544 & -0.04555\end{array}$

IM-2S cis a

$\begin{array}{llll}\mathrm{C} & -1.45221 & 0.40185 & 0.00004 \\ \mathrm{H} & -2.13461 & 1.27030 & 0.00021 \\ \mathrm{C} & -0.02432 & 0.73154 & -0.00003 \\ \mathrm{C} & 0.86761 & -0.26011 & 0.00002 \\ \mathrm{H} & 0.28949 & 1.76575 & -0.00006 \\ \mathrm{H} & 0.52788 & -1.29098 & 0.00009 \\ \mathrm{O} & 2.19112 & -0.04387 & -0.00003 \\ \mathrm{H} & 2.66617 & -0.87787 & 0.00024 \\ \mathrm{O} & -1.90305 & -0.71948 & -0.00004\end{array}$

TS-2S b

$\begin{array}{lrrr}\mathrm{C} & 1.17245 & 0.29642 & 0.35685 \\ \mathrm{H} & 1.59968 & 0.60139 & 1.32737 \\ \mathrm{C} & 0.09330 & 1.04930 & -0.20419 \\ \mathrm{C} & -1.20569 & 0.45382 & -0.15662 \\ \mathrm{H} & -1.50063 & 1.13640 & 0.69246 \\ \mathrm{H} & -1.87600 & 0.75389 & -0.96545 \\ \mathrm{O} & -1.38910 & -0.88567 & 0.20176 \\ \mathrm{H} & -0.60935 & -1.38292 & -0.07160 \\ \mathrm{O} & 1.64235 & -0.60257 & -0.32164\end{array}$

IM- $2 \mathrm{~S}$ cis b

$\begin{array}{llll}\mathrm{C} & -1.23389 & 0.34362 & -0.00001 \\ \mathrm{H} & -2.17781 & 0.90860 & -0.00010 \\ \mathrm{C} & -0.00814 & 1.09757 & 0.00004 \\ \mathrm{C} & 1.17400 & 0.43513 & -0.00002 \\ \mathrm{H} & -0.02786 & 2.17478 & 0.00007 \\ \mathrm{O} & 1.29068 & -0.87331 & 0.00002 \\ \mathrm{H} & 2.12726 & 0.95280 & -0.00020 \\ \mathrm{O} & -1.27580 & -0.88354 & -0.00001 \\ \mathrm{H} & 0.36755 & -1.23928 & -0.00001\end{array}$


IM-2S trans a

$\begin{array}{llll}\mathrm{C} & -1.06865 & -0.34383 & 0.00020 \\ \mathrm{H} & -0.64956 & -1.36378 & 0.00048 \\ \mathrm{C} & -0.09325 & 0.74757 & 0.00031 \\ \mathrm{C} & 1.22494 & 0.54464 & -0.00011 \\ \mathrm{H} & -0.47146 & 1.75901 & 0.00014 \\ \mathrm{H} & 1.92227 & 1.37559 & -0.00054 \\ \mathrm{O} & 1.77350 & -0.68830 & 0.00003 \\ \mathrm{H} & 2.73068 & -0.63021 & -0.00058 \\ \mathrm{O} & -2.26228 & -0.16557 & -0.00026\end{array}$

TS-3S isomer a

$\begin{array}{lrrr}\mathrm{C} & 1.35826 & 0.25595 & 0.19060 \\ \mathrm{C} & 0.05078 & 0.47413 & -0.22507 \\ \mathrm{C} & -1.02585 & -0.40545 & 0.12053 \\ \mathrm{O} & -2.33367 & 0.05406 & -0.00593 \\ \mathrm{H} & -0.74860 & -1.12723 & -0.70283 \\ \mathrm{H} & -0.94709 & -0.98544 & 1.04158 \\ \mathrm{H} & 1.28466 & 0.90508 & 1.13304 \\ \mathrm{O} & 2.38811 & -0.24888 & -0.16869 \\ \mathrm{H} & -2.32363 & 0.81838 & -0.59123\end{array}$

IM-2S trans b

$\begin{array}{llll}\mathrm{C} & 1.08345 & -0.37142 & -0.00010 \\ \mathrm{C} & -0.03725 & 0.35423 & 0.00006 \\ \mathrm{O} & 2.34199 & 0.08259 & 0.00005 \\ \mathrm{H} & -0.03238 & 1.43757 & -0.00024 \\ \mathrm{H} & 1.05357 & -1.45505 & -0.00037 \\ \mathrm{C} & -1.32586 & -0.32819 & 0.00007 \\ \mathrm{H} & -1.26448 & -1.43473 & 0.00029 \\ \mathrm{O} & -2.39515 & 0.22722 & -0.00004 \\ \mathrm{H} & 2.34646 & 1.04608 & 0.00006\end{array}$

TS-3S isomer b

$\begin{array}{lrrr}\mathrm{C} & 1.14386 & 0.17735 & 0.29495 \\ \mathrm{C} & 0.05845 & 1.00825 & 0.00292 \\ \mathrm{C} & -1.25605 & 0.46864 & -0.14352 \\ \mathrm{H} & -2.07979 & 1.10058 & 0.18042 \\ \mathrm{H} & -1.16589 & 0.68949 & -1.25605 \\ \mathrm{O} & -1.58228 & -0.86016 & 0.12545 \\ \mathrm{H} & 1.12322 & 0.30105 & 1.42906 \\ \mathrm{O} & 1.99376 & -0.46299 & -0.27049 \\ \mathrm{H} & -0.84695 & -1.43143 & -0.11922\end{array}$


TS-3S isomer c

$\begin{array}{lrrr}\mathrm{C} & -1.18189 & -0.34437 & -0.06453 \\ \mathrm{C} & 0.09482 & -0.54934 & -0.59599 \\ \mathrm{C} & 1.25151 & -0.54447 & 0.33103 \\ \mathrm{H} & 2.10828 & -1.14718 & 0.04068 \\ \mathrm{O} & 1.41073 & 0.84428 & 0.00496 \\ \mathrm{H} & 1.06631 & -0.61142 & 1.40088 \\ \mathrm{H} & -1.43932 & -1.45894 & 0.03448 \\ \mathrm{O} & -1.97960 & 0.52227 & 0.16521 \\ \mathrm{H} & 1.82909 & 0.91422 & -0.86037\end{array}$

IM-3S isomer b

$\begin{array}{llll}\mathrm{C} & 1.33249 & 0.44898 & -0.08385 \\ \mathrm{C} & 0.05221 & 1.09686 & -0.07674 \\ \mathrm{H} & 0.02896 & 2.17449 & -0.14756 \\ \mathrm{C} & -1.23266 & 0.36725 & 0.13215 \\ \mathrm{O} & -1.20682 & -1.00115 & -0.14736 \\ \mathrm{H} & -1.54708 & 0.56367 & 1.17202 \\ \mathrm{H} & -2.00041 & 0.81837 & -0.50272 \\ \mathrm{O} & 1.57739 & -0.70736 & 0.08766 \\ \mathrm{H} & -0.35824 & -1.36689 & 0.12641\end{array}$

IM-3S isomer a

$\begin{array}{lrrr}\mathrm{C} & -1.52452 & 0.51327 & 0.00006 \\ \mathrm{C} & -0.10274 & 0.72741 & -0.00019 \\ \mathrm{H} & 0.29887 & 1.72927 & -0.00003 \\ \mathrm{C} & 0.82928 & -0.42730 & -0.00017 \\ \mathrm{H} & 0.61461 & -1.05035 & 0.87904 \\ \mathrm{H} & 0.61503 & -1.05013 & -0.87963 \\ \mathrm{O} & 2.15131 & 0.05731 & 0.00023 \\ \mathrm{H} & 2.75955 & -0.68448 & -0.00017 \\ \mathrm{O} & -2.08883 & -0.53538 & 0.00009\end{array}$

TS-6S

$\begin{array}{llll}\mathrm{C} & -1.25239 & 0.26655 & 0.41130 \\ \mathrm{H} & -1.53557 & 1.41618 & 0.26135 \\ \mathrm{O} & -2.07118 & -0.63763 & -0.24181 \\ \mathrm{H} & -1.23436 & 0.14693 & 1.49389 \\ \mathrm{C} & -0.18554 & 0.92772 & -0.21962 \\ \mathrm{H} & -0.34442 & 0.84989 & -1.30371 \\ \mathrm{C} & 1.63936 & -0.31435 & -0.27158 \\ \mathrm{O} & 2.65503 & -0.20651 & 0.20141 \\ \mathrm{H} & -2.76502 & -0.93942 & 0.35111\end{array}$




\begin{tabular}{lrrr} 
& \multicolumn{3}{c}{$\mathrm{CHOHCH}_{2}+\mathrm{CO}$} \\
$\mathrm{C}$ & -1.27409 & -0.13819 & 0.01359 \\
$\mathrm{H}$ & -1.84664 & -0.38202 & -0.87158 \\
$\mathrm{H}$ & -1.67795 & -0.46029 & 0.96770 \\
$\mathrm{C}$ & 0.03246 & 0.48565 & -0.08338 \\
$\mathrm{H}$ & 0.19311 & 1.50434 & 0.25545 \\
$\mathrm{O}$ & 1.10781 & -0.36241 & 0.00676 \\
$\mathrm{H}$ & 1.91876 & 0.15250 & 0.01313 \\
$\mathrm{C}$ & 0.00000 & 0.00000 & -0.64106 \\
$\mathrm{O}$ & 0.00000 & 0.00000 & 0.48079
\end{tabular}

TS-8S

$\begin{array}{lrrr}\text { C } & -1.25963 & 0.23199 & 0.07351 \\ \text { H } & -0.39817 & 0.90091 & 1.13110 \\ \text { C } & -0.03015 & 0.87821 & -0.10289 \\ \text { C } & 0.91981 & -0.11286 & -0.49744 \\ \text { H } & 0.55192 & -0.99673 & -0.99844 \\ \text { O } & 2.06295 & -0.24133 & 0.25978 \\ \text { O } & -2.07534 & -0.59651 & 0.07396 \\ \text { H } & 0.00206 & 1.96139 & -0.20773 \\ \text { H } & 2.16315 & -1.14693 & 0.56610\end{array}$

Cyclopropanone-2-ol

TS-10S

$\begin{array}{lrrrrrrr}\mathrm{C} & -0.76993 & -0.02738 & 0.07589 & \mathrm{C} & 1.35826 & 0.25587 & 0.19055 \\ \mathrm{C} & 0.63883 & 0.08619 & 0.48620 & \mathrm{H} & 1.28439 & 0.90514 & 1.13289 \\ \mathrm{C} & -0.11718 & 1.25337 & -0.20239 & \mathrm{C} & 0.05076 & 0.47377 & -0.22509 \\ \mathrm{H} & 0.86020 & 0.24834 & 1.53726 & \mathrm{O} & 2.38830 & -0.24868 & -0.16858 \\ \mathrm{O} & 1.63106 & -0.54331 & -0.25996 & \mathrm{C} & -1.02598 & -0.40560 & 0.12050 \\ \mathrm{H} & 0.13695 & 1.43826 & -1.23957 & \mathrm{O} & -2.33371 & 0.05416 & -0.00610 \\ \mathrm{H} & -0.32202 & 2.13072 & 0.39614 & \mathrm{H} & -0.94741 & -0.98580 & 1.04144 \\ \mathrm{O} & -1.71842 & -0.73115 & -0.07274 & \mathrm{H} & -0.74879 & -1.12725 & -0.70300 \\ \mathrm{H} & 1.51341 & -1.49469 & -0.19043 & \mathrm{H} & -2.32312 & 0.81982 & -0.58966\end{array}$


TS-11S

$\begin{array}{llll}\mathrm{C} & 1.47317 & -0.36098 & -0.00075 \\ \mathrm{C} & -0.11346 & 0.80854 & -0.00048 \\ \mathrm{C} & -1.12791 & 0.10414 & -0.00016 \\ \mathrm{H} & 0.30995 & -1.62579 & -0.00109 \\ \mathrm{H} & 0.39365 & 1.74670 & -0.00072 \\ \mathrm{H} & -0.49371 & -1.40207 & -0.00062 \\ \mathrm{O} & -2.43353 & -0.08592 & 0.00056 \\ \mathrm{O} & 2.56385 & -0.03959 & 0.00079 \\ \mathrm{H} & -2.64320 & -1.02504 & -0.00004\end{array}$

TS- $12 \mathrm{~S}$ isomer a

$\begin{array}{llll}\mathrm{C} & -0.99652 & 0.17775 & -0.13235 \\ \mathrm{H} & -0.30643 & 2.05597 & 0.40395 \\ \mathrm{C} & 0.00816 & 1.20171 & -0.19143 \\ \mathrm{C} & 0.91036 & 0.26787 & 0.51950 \\ \mathrm{O} & 1.33482 & -0.83928 & -0.21978 \\ \mathrm{H} & 0.71755 & 0.08028 & 1.56581 \\ \mathrm{O} & -1.63654 & -0.77073 & 0.05039 \\ \mathrm{H} & 0.26970 & 1.50568 & -1.20380 \\ \mathrm{H} & 2.20094 & -0.64573 & -0.58519\end{array}$

$\mathrm{COHCH}+\mathrm{H}_{2}+\mathrm{CO}$

$\begin{array}{llll}\text { C } & -1.31737 & 0.01676 & 0.00016\end{array}$

$\begin{array}{llll}\text { C } & -0.12357 & -0.01245 & -0.00037\end{array}$

$\begin{array}{llll}\mathrm{H} & -2.37721 & 0.04264 & 0.00015\end{array}$

$\begin{array}{llll}\text { O } & 1.18088 & -0.10529 & 0.00013\end{array}$

\begin{tabular}{llll}
$\mathrm{H}$ & 1.57574 & 0.77384 & 0.00008 \\
\hline
\end{tabular}

$\begin{array}{llll}\text { C } & 0.00000 & 0.00000 & -0.64106\end{array}$

\begin{tabular}{llll}
$\mathrm{O}$ & 0.00000 & 0.00000 & 0.48079 \\
\hline
\end{tabular}

$\begin{array}{llll}\mathrm{H} & 0.00000 & 0.00000 & 0.36915\end{array}$

$\begin{array}{llll}\mathrm{H} & 0.00000 & 0.00000 & -0.36915\end{array}$
TS- $12 \mathrm{~S}$ isomer b

$\begin{array}{llll}\text { C } & 0.97792 & 0.26526 & -0.06470\end{array}$

$\mathrm{H} \quad-0.27240 \quad 1.50277 \quad-1.24131$

$\begin{array}{llll}\text { C } & -0.08078 & 1.25869 & -0.19725\end{array}$

$\begin{array}{llll}\text { C } & -0.88742 & 0.24059 & 0.45645\end{array}$

$\mathrm{H} \quad \begin{array}{llll}-0.89860 & 0.18326 & 1.53495\end{array}$

$\begin{array}{llll}\mathrm{O} & -1.31434 & -0.83940 & -0.27620\end{array}$

$\begin{array}{llll}\text { O } & 1.52732 & -0.76404 & 0.05887\end{array}$

$\begin{array}{llll}\mathrm{H} & 0.12767 & 2.14586 & 0.39209\end{array}$

$\begin{array}{llll}\mathrm{H} & -0.71885 & -1.59163 & -0.11412\end{array}$ 


\begin{tabular}{lrrr}
\multicolumn{4}{c}{$\mathrm{CHOHCH}_{2}+\mathrm{CO}$} \\
$\mathrm{C}$ & -1.27409 & -0.13819 & 0.01359 \\
$\mathrm{H}$ & -1.84664 & -0.38202 & -0.87158 \\
$\mathrm{H}$ & -1.67795 & -0.46029 & 0.96770 \\
$\mathrm{C}$ & 0.03246 & 0.48565 & -0.08338 \\
$\mathrm{H}$ & 0.19311 & 1.50434 & 0.25545 \\
$\mathrm{O}$ & 1.10781 & -0.36241 & 0.00676 \\
$\mathrm{H}$ & 1.91876 & 0.15250 & 0.01313 \\
\hline $\mathrm{C}$ & 0.00000 & 0.00000 & -0.64106 \\
$\mathrm{O}$ & 0.00000 & 0.00000 & 0.48079
\end{tabular}

\section{M06-2x/cc-pVTZ Cartesian Coordinates for the Novel HCO/HOC Pathways, Triplet Surface of $\mathbf{O}\left({ }^{3} \mathrm{P}\right)+$ Propargyl Alcohol}

\begin{tabular}{cccc}
\multicolumn{4}{c}{ TS-HCO to HOC } \\
C & 0.06901 & 0.73038 & 0.00000 \\
H & -0.96612 & -0.09097 & 0.00000 \\
O & 0.06901 & -0.53641 & 0.00000 \\
& & & \\
& & HOC & \\
C & 0.05965 & 0.78801 & 0.00000 \\
O & 0.05965 & -0.48065 & 0.00000 \\
H & -0.83507 & -0.88287 & 0.00000
\end{tabular}

TS-8T

$\begin{array}{lrrr}\mathrm{C} & -1.13825 & -0.54938 & -0.15550 \\ \mathrm{H} & -2.00300 & -1.14778 & -0.42111 \\ \mathrm{C} & 0.12643 & -1.08244 & -0.10180 \\ \mathrm{C} & 1.34543 & -0.28287 & 0.25144 \\ \mathrm{H} & 1.45057 & -0.25457 & 1.34586 \\ \mathrm{H} & 2.24017 & -0.75620 & -0.17203 \\ \mathrm{O} & 1.00564 & 0.89532 & -0.34180 \\ \mathrm{H} & -0.49277 & 1.12392 & 0.17416 \\ \mathrm{O} & -1.40522 & 0.67003 & 0.23033\end{array}$


IM-5T

$\begin{array}{llll}\mathrm{C} & 1.16525 & 0.52955 & -0.21783 \\ \mathrm{H} & 1.97913 & 1.10675 & -0.63795 \\ \mathrm{C} & -0.06472 & 1.00091 & -0.10495 \\ \mathrm{C} & -1.30567 & 0.32737 & 0.34905 \\ \mathrm{H} & -1.25660 & 0.12548 & 1.42701 \\ \mathrm{H} & -2.17049 & 0.96854 & 0.13877 \\ \mathrm{O} & -1.33793 & -0.83100 & -0.41041 \\ \mathrm{H} & 0.79656 & -1.24388 & 0.35631 \\ \mathrm{O} & 1.57321 & -0.68198 & 0.23019\end{array}$

IM-6T

$\begin{array}{llll}\mathrm{C} & -1.23352 & 0.47743 & 0.00013 \\ \mathrm{H} & -2.14296 & 1.05289 & 0.00013 \\ \mathrm{C} & 0.09868 & 1.08083 & -0.00003 \\ \mathrm{C} & 1.28810 & 0.33497 & -0.00003 \\ \mathrm{H} & 2.24159 & 0.86678 & -0.00014 \\ \mathrm{O} & 1.26381 & -0.93221 & 0.00007 \\ \mathrm{H} & -0.43698 & -1.21736 & -0.00007 \\ \mathrm{O} & -1.35600 & -0.84564 & -0.00010 \\ \mathrm{H} & 0.15626 & 2.16116 & -0.00014\end{array}$

TS-9T

$\begin{array}{lrrr}\mathrm{C} & 1.22392 & 0.51043 & 0.03295 \\ \mathrm{H} & 2.15044 & 1.06807 & 0.03690 \\ \mathrm{C} & -0.01830 & 1.09873 & 0.08255 \\ \mathrm{C} & -1.27072 & 0.37065 & 0.03172 \\ \mathrm{H} & -2.19914 & 0.90484 & 0.24544 \\ \mathrm{H} & -0.90370 & 0.95115 & -1.08447 \\ \mathrm{O} & -1.20806 & -0.90298 & 0.02058 \\ \mathrm{H} & 0.37160 & -1.14852 & -0.01570 \\ \mathrm{O} & 1.32948 & -0.80382 & -0.02877\end{array}$

TS-10T

$\begin{array}{lrrr}\mathrm{C} & 1.38301 & 0.27265 & 0.05697 \\ \mathrm{H} & 2.40057 & 0.55252 & 0.32396 \\ \mathrm{C} & 0.47571 & 1.20902 & -0.11767 \\ \mathrm{C} & -1.59898 & 0.42475 & 0.24481 \\ \mathrm{H} & -1.92398 & 1.24938 & -0.43454 \\ \mathrm{O} & -1.58608 & -0.72258 & -0.07007 \\ \mathrm{H} & 0.30107 & -1.27226 & -0.16521 \\ \mathrm{O} & 1.23367 & -1.05877 & -0.01861 \\ \mathrm{H} & 0.48318 & 2.28268 & -0.11940\end{array}$




\begin{tabular}{lrrrrrrr} 
& \multicolumn{3}{c}{$\mathrm{CH}_{2} \mathrm{CH}_{2} \mathrm{OH}+\mathrm{HCO}$} & & \multicolumn{3}{c}{ TS-11T cis a } \\
$\mathrm{C}$ & -1.55016 & 0.12400 & -0.09486 & $\mathrm{C}$ & -0.93376 & -0.34099 & -0.09741 \\
$\mathrm{H}$ & -2.57170 & 0.18181 & -0.46395 & $\mathrm{H}$ & -0.27730 & 1.72535 & 0.14104 \\
$\mathrm{C}$ & -0.89817 & 1.21044 & 0.24511 & $\mathrm{C}$ & 0.08221 & 0.71468 & -0.01249 \\
$\mathrm{C}$ & 1.81210 & 0.41311 & -0.44726 & $\mathrm{C}$ & 1.48323 & 0.37835 & 0.07398 \\
$\mathrm{H}$ & 2.01160 & 1.47163 & -0.15017 & $\mathrm{O}$ & 1.90643 & -0.76236 & 0.02126 \\
$\mathrm{O}$ & 1.82625 & -0.49842 & 0.29170 & $\mathrm{H}$ & 2.16178 & 1.24320 & 0.15620 \\
$\mathrm{H}$ & -0.19613 & -1.17684 & 0.24147 & $\mathrm{O}$ & -2.25230 & -0.17375 & -0.00435 \\
$\mathrm{O}$ & -1.11388 & -1.15424 & -0.05697 & $\mathrm{H}$ & -0.36671 & 0.31165 & -1.12708 \\
$\mathrm{H}$ & -1.12529 & 2.25930 & 0.27690 & $\mathrm{H}$ & -2.54093 & -0.30363 & 0.91007
\end{tabular}

TS-11T cis b

TS-11T trans a

$\begin{array}{llllllll}\mathrm{C} & 1.48591 & 0.36947 & -0.07284 & \mathrm{C} & -1.07122 & -0.33852 & 0.13051 \\ \mathrm{H} & 2.17416 & 1.22593 & -0.15988 & \mathrm{H} & -0.61914 & -1.28776 & 0.46927 \\ \mathrm{C} & 0.09023 & 0.72238 & 0.03981 & \mathrm{C} & -0.14480 & 0.76696 & -0.02920 \\ \mathrm{C} & -0.93502 & -0.31180 & 0.11854 & \mathrm{C} & 1.30351 & 0.57986 & 0.00923 \\ \mathrm{H} & -0.25596 & 1.74010 & -0.10068 & \mathrm{H} & -0.52770 & 1.72073 & -0.36708 \\ \mathrm{O} & -2.21919 & -0.15570 & -0.20929 & \mathrm{H} & 0.51921 & 0.85678 & 1.06432 \\ \mathrm{H} & -0.37587 & 0.35554 & 1.16163 & \mathrm{O} & 1.94891 & -0.57465 & -0.18807 \\ \mathrm{O} & 1.89539 & -0.77741 & -0.02493 & \mathrm{H} & 2.58768 & -0.74595 & 0.51229 \\ \mathrm{H} & -2.79861 & -0.53701 & 0.45962 & \mathrm{O} & -2.25954 & -0.24956 & -0.10468\end{array}$


TS-11T trans b

$\begin{array}{llll}\mathrm{C} & 1.07122 & -0.33852 & 0.13049 \\ \mathrm{H} & 0.61913 & -1.28778 & 0.46920 \\ \mathrm{C} & 0.14480 & 0.76696 & -0.02920 \\ \mathrm{C} & -1.30351 & 0.57987 & 0.00920 \\ \mathrm{H} & 0.52770 & 1.72073 & -0.36704 \\ \mathrm{O} & -1.94891 & -0.57465 & -0.18806 \\ \mathrm{H} & -0.51923 & 0.85677 & 1.06431 \\ \mathrm{O} & 2.25954 & -0.24955 & -0.10466 \\ \mathrm{H} & -2.58766 & -0.74595 & 0.51232\end{array}$

IM-7T cis b

$\begin{array}{lrrr}\mathrm{C} & 1.48690 & 0.32301 & 0.01740 \\ \mathrm{H} & 2.23260 & 1.13973 & 0.04264 \\ \mathrm{C} & 0.05330 & 0.78299 & 0.00755 \\ \mathrm{C} & -0.90801 & -0.33483 & -0.09349 \\ \mathrm{H} & -0.06148 & 1.49707 & -0.82129 \\ \mathrm{O} & -2.23761 & -0.18832 & -0.06504 \\ \mathrm{H} & -0.10306 & 1.37732 & 0.92104 \\ \mathrm{O} & 1.83160 & -0.82349 & 0.00507 \\ \mathrm{H} & -2.61308 & -0.54667 & 0.74866\end{array}$

IM-7T cis a

$\begin{array}{llll}\mathrm{C} & -1.10670 & 0.50617 & -0.32528 \\ \mathrm{H} & 0.06667 & 1.06438 & 1.37521 \\ \mathrm{C} & 0.10862 & 1.10634 & 0.28182 \\ \mathrm{C} & 1.18163 & 0.17271 & -0.24323 \\ \mathrm{O} & 1.29337 & -0.95959 & 0.15725 \\ \mathrm{H} & 1.78519 & 0.53586 & -1.09250 \\ \mathrm{O} & -1.58561 & -0.67707 & 0.02835 \\ \mathrm{H} & 0.25967 & 2.12621 & -0.06112 \\ \mathrm{H} & -0.87491 & -1.34450 & 0.01371\end{array}$

IM-7T trans a

$\begin{array}{llll}\mathrm{C} & -1.09608 & -0.35385 & 0.18419 \\ \mathrm{H} & -0.70299 & -1.33111 & 0.52079 \\ \mathrm{C} & -0.08814 & 0.78366 & 0.21297 \\ \mathrm{C} & 1.16624 & 0.37723 & -0.46831 \\ \mathrm{H} & -0.53346 & 1.65053 & -0.27036 \\ \mathrm{H} & 0.12063 & 1.03189 & 1.26305 \\ \mathrm{O} & 2.02809 & -0.52783 & 0.00680 \\ \mathrm{H} & 2.80481 & -0.11028 & 0.40164 \\ \mathrm{O} & -2.22572 & -0.23257 & -0.19283\end{array}$


IM-7T trans b

$\begin{array}{lrrrrrrr}\mathrm{C} & 1.09608 & -0.35386 & 0.18419 & \mathrm{C} & -1.31748 & 0.59673 & -0.55715 \\ \mathrm{H} & 0.70298 & -1.33112 & 0.52078 & \mathrm{H} & 0.42350 & 1.09533 & 1.36469 \\ \mathrm{C} & 0.08814 & 0.78366 & 0.21297 & \mathrm{C} & 0.82306 & 1.14338 & 0.36266 \\ \mathrm{C} & -1.16625 & 0.37725 & -0.46830 & \mathrm{C} & 1.19754 & -0.07602 & -0.30716 \\ \mathrm{H} & 0.53347 & 1.65053 & -0.27036 & \mathrm{O} & 0.93930 & -1.18896 & 0.12965 \\ \mathrm{O} & -2.02809 & -0.52784 & 0.00678 & \mathrm{H} & 1.73053 & 0.03600 & -1.26289 \\ \mathrm{H} & -0.12061 & 1.03190 & 1.26304 & \mathrm{O} & -1.71376 & -0.31705 & 0.23020 \\ \mathrm{O} & 2.22572 & -0.23258 & -0.19283 & \mathrm{H} & 1.06079 & 2.10221 & -0.07066 \\ \mathrm{H} & -2.80473 & -0.11031 & 0.40179 & \mathrm{H} & -1.23788 & -1.16994 & 0.10002\end{array}$


IM-8T

$\begin{array}{llllllll}\mathrm{C} & -1.10461 & 0.50387 & -0.37458 & \mathrm{C} & -1.31766 & 0.59672 & -0.55699 \\ \mathrm{C} & 0.17077 & 1.11905 & 0.04889 & \mathrm{C} & 0.82305 & 1.14341 & 0.36267 \\ \mathrm{H} & 0.11647 & 1.55831 & 1.05798 & \mathrm{H} & 0.42361 & 1.09523 & 1.36475 \\ \mathrm{H} & 0.45871 & 1.93354 & -0.61865 & \mathrm{H} & 1.06018 & 2.10232 & -0.07079 \\ \mathrm{C} & 1.29695 & 0.10818 & 0.08596 & \mathrm{C} & 1.19765 & -0.07591 & -0.30722 \\ \mathrm{O} & 1.14979 & -1.06534 & -0.12335 & \mathrm{O} & 0.93972 & -1.18888 & 0.12968 \\ \mathrm{O} & -1.64393 & -0.56582 & 0.20473 & \mathrm{O} & -1.71394 & -0.31722 & 0.23017 \\ \mathrm{H} & 2.29356 & 0.51161 & 0.34443 & \mathrm{H} & 1.73032 & 0.03621 & -1.26312 \\ \mathrm{H} & -1.09428 & -1.34073 & 0.00361 & \mathrm{H} & -1.23850 & -1.17026 & 0.09950\end{array}$

\begin{tabular}{lrrrrrrr}
\multicolumn{5}{c}{$\mathrm{HOC}+\mathrm{CH}_{2} \mathrm{CHO}$} & & & \multicolumn{2}{c}{$\mathrm{TS}-15 \mathrm{~T}$} \\
$\mathrm{C}$ & 1.99787 & 0.86125 & 0.00066 & $\mathrm{C}$ & -1.15762 & 0.12897 & -0.35985 \\
$\mathrm{H}$ & -0.39298 & 1.52429 & 0.00018 & $\mathrm{C}$ & -0.69161 & 1.21457 & 0.27466 \\
$\mathrm{C}$ & -1.39709 & 1.11085 & -0.00020 & $\mathrm{H}$ & -0.51095 & 1.18009 & 1.34434 \\
$\mathrm{C}$ & -1.56859 & -0.29844 & -0.00002 & $\mathrm{H}$ & -0.75006 & 2.18362 & -0.19790 \\
$\mathrm{O}$ & -0.62907 & -1.09967 & 0.00040 & $\mathrm{C}$ & 1.32568 & 0.49921 & -0.20175 \\
$\mathrm{H}$ & -2.59155 & -0.69368 & -0.00024 & $\mathrm{O}$ & 1.52125 & -0.60454 & 0.20902 \\
$\mathrm{O}$ & 1.89387 & -0.39029 & -0.00063 & $\mathrm{O}$ & -1.12376 & -1.12820 & -0.01031 \\
$\mathrm{H}$ & -2.26079 & 1.75871 & -0.00060 & $\mathrm{H}$ & 1.42535 & 0.80288 & -1.26436 \\
$\mathrm{H}$ & 0.93373 & -0.71165 & -0.00011 & $\mathrm{H}$ & -0.20286 & -1.36114 & 0.24989
\end{tabular}




\begin{tabular}{lrrrrrrr} 
& \multicolumn{2}{c}{$\mathrm{HCO}+\mathrm{CH}_{2} \mathrm{COH}$} & & & \multicolumn{2}{c}{$\mathrm{TS}-16 \mathrm{~T}$} \\
$\mathrm{C}$ & -1.11874 & -0.09344 & -0.37727 & $\mathrm{C}$ & 1.54822 & 0.42070 & -0.14920 \\
$\mathrm{C}$ & -1.97778 & 0.79887 & 0.06242 & $\mathrm{C}$ & 0.16682 & 0.96036 & 0.05178 \\
$\mathrm{H}$ & -2.39694 & 0.71403 & 1.06048 & $\mathrm{H}$ & -0.15776 & 1.50738 & -0.83981 \\
$\mathrm{H}$ & -2.28441 & 1.62063 & -0.56573 & $\mathrm{H}$ & 0.20663 & 1.68793 & 0.87020 \\
$\mathrm{C}$ & 1.95141 & 0.89060 & 0.09474 & $\mathrm{C}$ & -0.79183 & -0.20200 & 0.33317 \\
$\mathrm{O}$ & 1.99257 & -0.26977 & -0.07112 & $\mathrm{H}$ & 0.55479 & -1.14847 & 0.17253 \\
$\mathrm{O}$ & -0.70599 & -1.23765 & 0.13956 & $\mathrm{O}$ & 1.56481 & -0.83309 & -0.01487 \\
$\mathrm{H}$ & 1.00840 & 1.46770 & 0.26493 & $\mathrm{O}$ & -1.99552 & -0.26711 & -0.23985 \\
$\mathrm{H}$ & 0.25099 & -1.31914 & 0.01353 & $\mathrm{H}$ & -2.69725 & -0.31955 & 0.42037
\end{tabular}

IM-9T

TS-17T-A

$\begin{array}{lrllllll}\mathrm{C} & 1.47422 & 0.34223 & -0.33737 & \mathrm{C} & 1.72148 & 0.29181 & -0.36769 \\ \mathrm{C} & 0.15298 & 1.01868 & 0.02540 & \mathrm{C} & -0.19334 & 1.19964 & 0.02452 \\ \mathrm{H} & -0.17519 & 1.60120 & -0.84148 & \mathrm{H} & -0.32288 & 1.58489 & -0.98217 \\ \mathrm{H} & 0.44288 & 1.75184 & 0.78576 & \mathrm{H} & 0.22663 & 1.88447 & 0.74771 \\ \mathrm{C} & -0.95245 & 0.15485 & 0.52377 & \mathrm{C} & -1.03416 & 0.22864 & 0.46079 \\ \mathrm{H} & 0.68295 & -1.16194 & 0.52258 & \mathrm{H} & 0.65968 & -0.95649 & 0.55257 \\ \mathrm{O} & 1.51751 & -0.87955 & 0.07117 & \mathrm{O} & 1.57818 & -0.83481 & 0.15539 \\ \mathrm{O} & -1.80267 & -0.49856 & -0.27762 & \mathrm{O} & -1.69094 & -0.68319 & -0.27813 \\ \mathrm{H} & -2.71790 & -0.26081 & -0.08610 & \mathrm{H} & -2.62523 & -0.68945 & -0.04198\end{array}$


TS-17T-B

$\begin{array}{lrrr}\mathrm{C} & 1.32560 & 0.17222 & -0.42349 \\ \mathrm{C} & 0.65828 & 1.22847 & 0.11707 \\ \mathrm{H} & 0.48389 & 2.11464 & -0.47673 \\ \mathrm{H} & 0.64013 & 1.34368 & 1.19586 \\ \mathrm{C} & -0.96050 & -0.28179 & 0.46903 \\ \mathrm{H} & 0.29384 & -1.09300 & 0.36442 \\ \mathrm{O} & 1.37552 & -1.00994 & 0.03433 \\ \mathrm{O} & -1.98526 & -0.17493 & -0.29964 \\ \mathrm{H} & -2.68026 & 0.40019 & 0.06329\end{array}$

$\mathrm{HOC}+\mathrm{CH}_{2} \mathrm{COH}-\mathrm{B}$

\begin{tabular}{|c|c|c|c|c|c|c|c|}
\hline \multicolumn{4}{|c|}{$\mathrm{HOC}+\mathrm{CH}_{2} \mathrm{COH}-\mathrm{B}$} & \multicolumn{4}{|c|}{ TS-18T } \\
\hline $\mathrm{C}$ & 1.53702 & 0.04161 & -0.37897 & $\mathrm{C}$ & -1.48059 & 0.46113 & -0.03668 \\
\hline $\mathrm{C}$ & 1.28877 & 1.22553 & 0.14314 & $\mathrm{H}$ & -2.07377 & 1.33847 & -0.31375 \\
\hline $\mathrm{H}$ & 1.53541 & 2.12653 & -0.39686 & $\mathrm{C}$ & -0.01211 & 0.63094 & -0.10740 \\
\hline $\mathrm{H}$ & 0.87940 & 1.31299 & 1.14652 & $\mathrm{C}$ & 0.97533 & -0.28759 & -0.03895 \\
\hline $\mathrm{C}$ & -1.44880 & -0.31256 & 0.49121 & $\mathrm{H}$ & -0.91512 & 0.82961 & 1.05696 \\
\hline $\mathrm{H}$ & 0.35095 & -1.25604 & 0.27220 & $\mathrm{H}$ & 0.73443 & -1.34599 & -0.04907 \\
\hline $\mathrm{O}$ & 1.29342 & -1.19184 & 0.01012 & $\mathrm{O}$ & 2.26929 & 0.07865 & 0.03215 \\
\hline $\mathrm{O}$ & -2.30629 & 0.10614 & -0.33309 & $\mathrm{H}$ & 2.83198 & -0.69859 & -0.01048 \\
\hline $\mathrm{H}$ & -2.92471 & 0.77462 & 0.02966 & $\mathrm{O}$ & -1.95345 & -0.69744 & 0.01965 \\
\hline
\end{tabular}

$\mathrm{HOC}+\mathrm{CH}_{2} \mathrm{COH}-\mathrm{A}$

$\begin{array}{llll}\mathrm{C} & -2.61937 & 0.22240 & 0.18607 \\ \mathrm{C} & 0.55601 & 1.15845 & 0.04708 \\ \mathrm{H} & 0.43361 & 1.32855 & 1.11284 \\ \mathrm{H} & 0.00821 & 1.78478 & -0.64086 \\ \mathrm{C} & 1.38419 & 0.23751 & -0.39573 \\ \mathrm{H} & -0.90627 & -0.44782 & -0.20821 \\ \mathrm{O} & -1.85293 & -0.73110 & -0.13064 \\ \mathrm{O} & 2.05840 & -0.70523 & 0.24422 \\ \mathrm{H} & 2.89566 & -0.88505 & -0.19693\end{array}$

$\begin{array}{llll}\text { C } & -2.61937 & 0.22240 & 0.18607\end{array}$

$\begin{array}{llll}\text { C } & 0.55601 & 1.15845 & 0.04708\end{array}$

$\begin{array}{llll}\mathrm{H} & 2.89566 & -0.88505 & -0.19693\end{array}$ 
IM-10T

$\begin{array}{lrrr}\mathrm{C} & 1.45659 & -0.53216 & 0.00006 \\ \mathrm{H} & 1.89732 & -1.05010 & -0.86734 \\ \mathrm{C} & -0.01806 & -0.61392 & -0.00026 \\ \mathrm{C} & -0.99053 & 0.26037 & 0.00004 \\ \mathrm{H} & 1.89695 & -1.05010 & 0.86763 \\ \mathrm{H} & -0.75709 & 1.32402 & 0.00034 \\ \mathrm{O} & -2.31181 & -0.07872 & 0.00020 \\ \mathrm{H} & -2.84192 & 0.71997 & -0.00122 \\ \mathrm{O} & 1.95139 & 0.75003 & -0.00000\end{array}$

IM-11T

$\begin{array}{lrllllll}\mathrm{C} & 1.44266 & 0.42963 & 0.00384 & \mathrm{C} & 1.87478 & 0.29427 & -0.06241 \\ \mathrm{H} & 1.91647 & 0.90134 & 0.88268 & \mathrm{H} & 2.06035 & 0.95644 & 0.80028 \\ \mathrm{C} & -0.03006 & 0.72056 & -0.03218 & \mathrm{C} & -0.27895 & 0.95864 & 0.00777 \\ \mathrm{C} & -0.93980 & -0.22577 & -0.01971 & \mathrm{C} & -0.99261 & -0.13917 & -0.06247 \\ \mathrm{H} & 1.96273 & 0.90715 & -0.84477 & \mathrm{H} & 2.00300 & 0.74893 & -1.05688 \\ \mathrm{O} & -2.26222 & -0.15377 & 0.08949 & \mathrm{O} & -2.30110 & -0.28816 & 0.09716 \\ \mathrm{H} & -2.67646 & -0.81695 & -0.47228 & \mathrm{H} & -2.65571 & -0.85254 & -0.60056 \\ \mathrm{O} & 1.80071 & -0.88352 & 0.01014 & \mathrm{O} & 1.73590 & -0.90445 & 0.07809 \\ \mathrm{H} & -0.34742 & 1.76025 & -0.07431 & \mathrm{H} & -0.50535 & 2.00564 & 0.15781\end{array}$

TS-19T

$\begin{array}{lrrr}\mathrm{C} & 1.43466 & -0.49417 & -0.07131 \\ \mathrm{H} & 1.71064 & -0.98793 & -1.01307 \\ \mathrm{C} & -0.03841 & -0.67602 & 0.12478 \\ \mathrm{C} & -0.98690 & 0.22757 & -0.10797 \\ \mathrm{H} & 1.99579 & -1.03606 & 0.70799 \\ \mathrm{H} & -0.34858 & 0.36452 & 0.96079 \\ \mathrm{O} & -2.31216 & 0.02216 & -0.01557 \\ \mathrm{H} & -2.75725 & 0.86113 & -0.16302 \\ \mathrm{O} & 1.93006 & 0.78460 & -0.00514\end{array}$

TS-20T 


\begin{tabular}{lrrr} 
& \multicolumn{3}{c}{$\mathrm{H}_{2} \mathrm{CO}+\mathrm{CHCOH}$} \\
$\mathrm{C}$ & -2.06671 & 0.29016 & 0.06903 \\
$\mathrm{H}$ & -2.20446 & 0.97705 & -0.78377 \\
$\mathrm{C}$ & 0.61089 & 1.17740 & -0.01763 \\
$\mathrm{C}$ & 1.02186 & -0.07240 & 0.04104 \\
$\mathrm{H}$ & -2.14134 & 0.74051 & 1.07299 \\
$\mathrm{O}$ & 2.27268 & -0.50651 & -0.08215 \\
$\mathrm{H}$ & 2.47654 & -1.12360 & 0.63123 \\
$\mathrm{O}$ & -1.85589 & -0.87874 & -0.08768 \\
$\mathrm{H}$ & 1.13877 & 2.11706 & -0.11646
\end{tabular}

M06-2x/cc-pVTZ Cartesian Coordinates for the Triplet State Surface of $\mathbf{O}\left({ }^{3} \mathbf{P}\right)+$ 3-Butyn-1-ol

\begin{tabular}{llll} 
& \multicolumn{3}{c}{ IM-0T, O(3P) +3-Butyn-1-ol } \\
C & 0.75511 & -1.22806 & -0.08594 \\
C & -0.69126 & -1.03761 & -0.08280 \\
C & -1.87993 & -0.86172 & -0.06164 \\
H & -2.93534 & -0.74154 & -0.04664 \\
$\mathrm{C}$ & 1.50203 & 0.00924 & 0.42678 \\
$\mathrm{H}$ & 1.00111 & -2.09112 & 0.53629 \\
$\mathrm{H}$ & 1.08832 & -1.44097 & -1.10277 \\
$\mathrm{H}$ & 2.56609 & -0.22097 & 0.46707 \\
$\mathrm{H}$ & 1.16582 & 0.24002 & 1.44291 \\
$\mathrm{O}$ & 1.35475 & 1.10996 & -0.43018 \\
$\mathrm{H}$ & 0.44752 & 1.42739 & -0.35480 \\
$\mathrm{O}$ & -1.53590 & 1.58205 & 0.16512
\end{tabular}


TS-1T

$\begin{array}{llll}\mathrm{C} & 0.78333 & -1.20711 & -0.12257 \\ \mathrm{C} & -0.65689 & -1.01772 & -0.08995 \\ \mathrm{C} & -1.82196 & -0.67183 & -0.02776 \\ \mathrm{H} & -2.88506 & -0.67690 & -0.00162 \\ \mathrm{C} & 1.51331 & 0.03879 & 0.41458 \\ \mathrm{H} & 1.04986 & -2.08021 & 0.47704 \\ \mathrm{H} & 1.10109 & -1.38844 & -1.15030 \\ \mathrm{H} & 2.58394 & -0.16263 & 0.40249 \\ \mathrm{H} & 1.20862 & 0.20888 & 1.45238 \\ \mathrm{O} & 1.29105 & 1.16316 & -0.38566 \\ \mathrm{H} & 0.36566 & 1.42484 & -0.27575 \\ \mathrm{O} & -1.58241 & 1.31455 & 0.14191\end{array}$

TS-2T

$\begin{array}{lrrr}\mathrm{C} & -0.65704 & 1.19447 & -0.15257 \\ \mathrm{C} & 0.77006 & 1.11345 & -0.19990 \\ \mathrm{C} & 1.72461 & 0.07347 & -0.01719 \\ \mathrm{C} & -1.45460 & 0.04788 & 0.43655 \\ \mathrm{H} & 0.26650 & 1.82970 & 0.67406 \\ \mathrm{H} & -1.13608 & 1.97173 & -0.73344 \\ \mathrm{H} & -2.49774 & 0.34766 & 0.51571 \\ \mathrm{H} & -1.08231 & -0.17456 & 1.44172 \\ \mathrm{O} & -1.39909 & -1.08432 & -0.39052 \\ \mathrm{H} & -0.49097 & -1.41976 & -0.33318 \\ \mathrm{O} & 1.38171 & -1.10194 & 0.14375 \\ \mathrm{H} & 2.78155 & 0.35981 & 0.00792\end{array}$

IM-1T

$\begin{array}{lrrr}\mathrm{C} & -0.76932 & 1.20385 & -0.12175 \\ \mathrm{C} & 0.69220 & 1.16818 & -0.14082 \\ \mathrm{C} & 1.71673 & 0.20488 & -0.00310 \\ \mathrm{C} & -1.42404 & -0.07397 & 0.44081 \\ \mathrm{H} & -1.09415 & 2.07245 & 0.46046 \\ \mathrm{H} & -1.13566 & 1.35461 & -1.14300 \\ \mathrm{H} & -2.49624 & 0.10673 & 0.51660 \\ \mathrm{H} & -1.03914 & -0.26325 & 1.44796 \\ \mathrm{O} & -1.24530 & -1.17623 & -0.40049 \\ \mathrm{H} & -0.32278 & -1.45297 & -0.30952 \\ \mathrm{O} & 1.50096 & -1.00025 & 0.15166 \\ \mathrm{H} & 2.74922 & 0.57661 & -0.03265\end{array}$

IM-2T

$\begin{array}{llll}\text { C } & -0.43853 & 1.06926 & -0.41825\end{array}$

$\begin{array}{llll}\text { C } & 0.94153 & 1.08852 & 0.03917\end{array}$

$\begin{array}{llll}\text { C } & 1.71626 & -0.11554 & 0.13122\end{array}$

$\begin{array}{llll}\text { C } & -1.49929 & 0.36162 & 0.37275\end{array}$

$\begin{array}{llll}\mathrm{H} & 1.40615 & 2.01232 & 0.37058\end{array}$

$\mathrm{H} \quad \begin{array}{llll}-0.62852 & 1.20626 & -1.47754\end{array}$

$\begin{array}{llll}\mathrm{H} & -2.46014 & 0.86101 & 0.24705\end{array}$

$\begin{array}{llll}\mathrm{H} & -1.24502 & 0.37506 & 1.43769\end{array}$

$\begin{array}{llll}\text { O } & -1.67283 & -0.96135 & -0.09793\end{array}$

$\mathrm{H} \quad-0.79404 \quad-1.36219 \quad-0.15187$

$\begin{array}{llll}\mathrm{O} & 1.25282 & -1.22663 & -0.10595\end{array}$

$\begin{array}{llll}\mathrm{H} & 2.76192 & -0.01171 & 0.45580\end{array}$ 
TS-3T

$\begin{array}{lrrrrrrr}\mathrm{C} & -0.47683 & 1.23615 & 0.09348 & \mathrm{C} & -0.50012 & 1.00891 & 0.49422 \\ \mathrm{C} & 0.94423 & 1.09005 & -0.14564 & \mathrm{C} & 0.76261 & 1.12857 & -0.24250 \\ \mathrm{C} & 1.64965 & -0.16425 & 0.00336 & \mathrm{C} & 1.67931 & -0.07501 & -0.12595 \\ \mathrm{C} & -1.54631 & 0.24823 & 0.38794 & \mathrm{C} & -1.72941 & 0.25759 & 0.18271 \\ \mathrm{H} & 1.52422 & 1.99467 & -0.27286 & \mathrm{H} & 1.33794 & 2.00271 & 0.07955 \\ \mathrm{H} & 0.14046 & 1.03378 & -1.10107 & \mathrm{H} & 0.59211 & 1.27256 & -1.32133 \\ \mathrm{H} & -2.51968 & 0.72850 & 0.28924 & \mathrm{H} & -2.36279 & 0.81262 & -0.51891 \\ \mathrm{H} & -1.43900 & -0.07692 & 1.43189 & \mathrm{H} & -2.31613 & 0.11142 & 1.09649 \\ \mathrm{O} & -1.51460 & -0.84878 & -0.49267 & \mathrm{O} & -1.43585 & -0.97577 & -0.45256 \\ \mathrm{H} & -0.68774 & -1.32083 & -0.30071 & \mathrm{H} & -0.71176 & -1.38952 & 0.03337 \\ \mathrm{O} & 1.11713 & -1.24150 & 0.25072 & \mathrm{O} & 1.37285 & -1.12414 & 0.36867 \\ \mathrm{H} & 2.73706 & -0.09806 & -0.14568 & \mathrm{H} & 2.69032 & 0.06914 & -0.54892\end{array}$

$\begin{array}{lrrrrrrr}\mathrm{C} & 0.53849 & 1.09143 & -0.41722 & \mathrm{C} & -1.03145 & 0.78183 & 0.53723 \\ \mathrm{C} & -0.33381 & 1.45046 & 0.52276 & \mathrm{C} & -0.78771 & 1.68116 & -0.37870 \\ \mathrm{C} & -1.75403 & -0.04773 & -0.18988 & \mathrm{C} & 2.00687 & 0.50071 & -0.05244 \\ \mathrm{C} & 1.43586 & -0.07683 & -0.57103 & \mathrm{C} & -1.15966 & -0.69137 & 0.59048 \\ \mathrm{H} & -0.93268 & 2.34796 & 0.42746 & \mathrm{H} & -0.74102 & 2.73978 & -0.15155 \\ \mathrm{H} & -0.26844 & 1.00828 & 1.51434 & \mathrm{H} & -0.64687 & 1.38622 & -1.41750 \\ \mathrm{H} & 2.46156 & 0.25345 & -0.73815 & \mathrm{H} & -2.17159 & -0.95600 & 0.89951 \\ \mathrm{H} & 1.13158 & -0.64943 & -1.45604 & \mathrm{H} & -0.47424 & -1.09170 & 1.34452 \\ \mathrm{O} & 1.43796 & -0.88419 & 0.58756 & \mathrm{O} & -0.94219 & -1.29177 & -0.67183 \\ \mathrm{H} & 0.57495 & -1.30954 & 0.65204 & \mathrm{H} & 0.00618 & -1.30430 & -0.83347 \\ \mathrm{O} & -1.46087 & -1.18039 & -0.00072 & \mathrm{O} & 1.90483 & -0.66173 & 0.07087 \\ \mathrm{H} & -2.10266 & 0.36193 & -1.16215 & \mathrm{H} & 2.15801 & 1.22002 & 0.78681\end{array}$


TS-5T

$\begin{array}{lrrr}\mathrm{C} & -0.71120 & 1.19499 & -0.09728 \\ \mathrm{C} & 0.55796 & 1.47653 & -0.17605 \\ \mathrm{C} & 1.95149 & -0.14135 & 0.05755 \\ \mathrm{C} & -1.28230 & -0.04160 & 0.56551 \\ \mathrm{H} & 1.18196 & 2.25255 & -0.58812 \\ \mathrm{H} & -1.42207 & 1.85697 & -0.59287 \\ \mathrm{H} & -2.22261 & 0.21446 & 1.05127 \\ \mathrm{H} & -0.59013 & -0.40387 & 1.32775 \\ \mathrm{O} & -1.57500 & -1.04030 & -0.38345 \\ \mathrm{H} & -0.73386 & -1.39271 & -0.69357 \\ \mathrm{O} & 1.38787 & -1.18237 & -0.07281 \\ \mathrm{H} & 2.18803 & 0.32260 & 1.04725\end{array}$

TS-6T

$\begin{array}{lrrrrrrr}\mathrm{C} & -0.87926 & 1.16667 & -0.07653 & \mathrm{C} & -0.61730 & 1.21720 & 0.03431 \\ \mathrm{C} & 0.59291 & 1.18413 & -0.24978 & \mathrm{C} & 0.84900 & 1.13607 & -0.22153 \\ \mathrm{C} & 1.60407 & 0.25386 & -0.13810 & \mathrm{C} & 1.68183 & -0.02445 & -0.07196 \\ \mathrm{C} & -1.36658 & -0.18919 & 0.46711 & \mathrm{C} & -1.31086 & -0.06507 & 0.47608 \\ \mathrm{H} & -1.19741 & 1.98617 & 0.56847 & \mathrm{H} & -0.78186 & 2.00482 & 0.77995 \\ \mathrm{H} & -1.32684 & 1.32198 & -1.06254 & \mathrm{H} & -1.09788 & 1.57808 & -0.88274 \\ \mathrm{H} & -2.44654 & -0.13047 & 0.60288 & \mathrm{H} & -2.34963 & 0.17036 & 0.70749 \\ \mathrm{H} & -0.91112 & -0.37552 & 1.44425 & \mathrm{H} & -0.84639 & -0.45193 & 1.38693 \\ \mathrm{O} & -1.11203 & -1.23275 & -0.43214 & \mathrm{O} & -1.32900 & -1.03831 & -0.54168 \\ \mathrm{H} & -0.19132 & -1.50604 & -0.33331 & \mathrm{H} & -0.49597 & -1.51770 & -0.50245 \\ \mathrm{O} & 1.68394 & -0.90923 & 0.21098 & \mathrm{O} & 1.40431 & -1.13687 & 0.26132 \\ \mathrm{H} & 1.79122 & 1.34687 & 0.53331 & \mathrm{H} & 1.35316 & 2.03525 & -0.54771\end{array}$

$\begin{array}{llll}\mathrm{C} & -1.23616 & 0.83187 & -0.24032 \\ \mathrm{C} & -0.43931 & 1.85007 & -0.07634 \\ \mathrm{C} & 2.15173 & 0.37956 & 0.24078 \\ \mathrm{C} & -1.18219 & -0.44245 & 0.56615 \\ \mathrm{H} & -0.28853 & 2.82275 & -0.51453 \\ \mathrm{H} & -1.99663 & 0.85512 & -1.02277 \\ \mathrm{H} & -2.14145 & -0.59394 & 1.06249 \\ \mathrm{H} & -0.41624 & -0.35697 & 1.34324 \\ \mathrm{O} & -0.97874 & -1.57203 & -0.25121 \\ \mathrm{H} & -0.08072 & -1.53028 & -0.59441 \\ \mathrm{O} & 1.90134 & -0.62460 & -0.30783 \\ \mathrm{H} & 1.77835 & 0.66204 & 1.25667\end{array}$

\section{IM-4T}


TS-7T

$\begin{array}{lrrr}\mathrm{C} & -1.19633 & 0.99177 & 0.13667 \\ \mathrm{C} & 0.12315 & 1.47046 & -0.30813 \\ \mathrm{C} & 2.03923 & 0.37851 & 0.00450 \\ \mathrm{C} & -1.23446 & -0.49047 & 0.51019 \\ \mathrm{H} & -1.53199 & 1.57588 & 1.00399 \\ \mathrm{H} & -1.92936 & 1.15627 & -0.66219 \\ \mathrm{H} & -2.23307 & -0.73751 & 0.86783 \\ \mathrm{H} & -0.53036 & -0.68859 & 1.32397 \\ \mathrm{O} & -0.99006 & -1.33052 & -0.59454 \\ \mathrm{H} & -0.05315 & -1.29998 & -0.80223 \\ \mathrm{O} & 1.92284 & -0.72912 & 0.22299 \\ \mathrm{H} & 0.42618 & 2.36941 & -0.81833\end{array}$

TS-8T

$\begin{array}{lrrrrrrr}\mathrm{C} & -0.51922 & 1.22029 & 0.03593 & \mathrm{C} & -0.28566 & 1.12960 & -0.29580 \\ \mathrm{C} & 0.92553 & 1.07429 & -0.25539 & \mathrm{C} & 1.18249 & 1.01130 & -0.03015 \\ \mathrm{C} & 1.65111 & -0.15945 & -0.11837 & \mathrm{C} & 1.67224 & -0.40832 & 0.14521 \\ \mathrm{C} & -1.35980 & 0.05363 & 0.45822 & \mathrm{C} & -1.29689 & 0.35481 & 0.47784 \\ \mathrm{H} & 0.56459 & 1.47475 & 0.84998 & \mathrm{H} & 1.48884 & 1.55320 & 0.87449 \\ \mathrm{H} & -0.96014 & 2.17290 & -0.21878 & \mathrm{H} & -0.62082 & 1.82875 & -1.04841 \\ \mathrm{H} & -2.37219 & 0.40283 & 0.65250 & \mathrm{H} & -2.14462 & 0.98964 & 0.73743 \\ \mathrm{H} & -0.97391 & -0.38332 & 1.38425 & \mathrm{H} & -0.85835 & -0.01236 & 1.41267 \\ \mathrm{O} & -1.44739 & -0.92912 & -0.55348 & \mathrm{O} & -1.84632 & -0.71937 & -0.27238 \\ \mathrm{H} & -0.63756 & -1.45042 & -0.51486 & \mathrm{H} & -1.11783 & -1.30925 & -0.49228 \\ \mathrm{O} & 1.29360 & -1.22619 & 0.28803 & \mathrm{O} & 1.07523 & -1.40660 & -0.03168 \\ \mathrm{H} & 1.42381 & 1.89314 & -0.75178 & \mathrm{H} & 1.78849 & 1.43349 & -0.83400\end{array}$

$\begin{array}{lrrr}\mathrm{C} & -1.59808 & 0.71165 & 0.16263 \\ \mathrm{C} & -0.51646 & 1.51835 & -0.41686 \\ \mathrm{C} & 2.25927 & 0.43168 & 0.64410 \\ \mathrm{C} & -1.10705 & -0.67938 & 0.56930 \\ \mathrm{H} & -2.02847 & 1.20393 & 1.04407 \\ \mathrm{H} & -2.40602 & 0.59196 & -0.56852 \\ \mathrm{H} & -1.92426 & -1.24337 & 1.01630 \\ \mathrm{H} & -0.31821 & -0.57712 & 1.32388 \\ \mathrm{O} & -0.66178 & -1.43064 & -0.53591 \\ \mathrm{H} & 0.03570 & -0.93431 & -0.97513 \\ \mathrm{O} & 2.26984 & -0.22328 & -0.26865 \\ \mathrm{H} & -0.44926 & 2.29643 & -1.15905\end{array}$

IM-5T 
TS-9T

$\begin{array}{llll}\mathrm{C} & -0.74489 & 0.89627 & 0.00622 \\ \mathrm{C} & 0.68442 & 1.18481 & -0.00151 \\ \mathrm{C} & 1.77621 & -0.65716 & 0.06243 \\ \mathrm{C} & -1.30347 & -0.48213 & 0.12374 \\ \mathrm{H} & 1.14167 & 1.57401 & 0.90544 \\ \mathrm{H} & -1.44679 & 1.71028 & 0.16068 \\ \mathrm{H} & -1.10092 & -0.89955 & 1.11706 \\ \mathrm{H} & -0.80538 & -1.15121 & -0.58837 \\ \mathrm{O} & -2.70661 & -0.50929 & -0.03065 \\ \mathrm{H} & -2.91892 & -0.16930 & -0.90334 \\ \mathrm{O} & 2.89595 & -0.52547 & -0.08492 \\ \mathrm{H} & 1.14207 & 1.56311 & -0.91217\end{array}$

TS-10T

$\begin{array}{lrrr}\mathrm{C} & -0.29489 & 0.85866 & -0.45936 \\ \mathrm{C} & 1.10930 & 1.03997 & 0.03948 \\ \mathrm{C} & 1.84126 & -0.28499 & 0.19938 \\ \mathrm{C} & -1.31328 & 0.27594 & 0.43730 \\ \mathrm{H} & 1.14576 & 1.53533 & 1.01361 \\ \mathrm{H} & -0.49279 & 0.77776 & -1.51878 \\ \mathrm{H} & -1.30733 & 1.51933 & 0.06594 \\ \mathrm{H} & -1.07892 & 0.21612 & 1.49462 \\ \mathrm{O} & -2.21346 & -0.63384 & -0.04452 \\ \mathrm{H} & -1.75950 & -1.18987 & -0.69212 \\ \mathrm{O} & 1.42838 & -1.34745 & -0.08551 \\ \mathrm{H} & 1.71910 & 1.65424 & -0.62384\end{array}$

$$
\mathrm{CO}+\mathrm{CH}_{2} \mathrm{CHCH}_{2} \mathrm{OH}
$$

$\begin{array}{lrrr}\mathrm{C} & -1.10111 & 0.97440 & 0.05822 \\ \mathrm{C} & 0.22877 & 1.54694 & -0.02932 \\ \mathrm{C} & 2.30989 & -0.91488 & 0.14122 \\ \mathrm{C} & -1.31943 & -0.49999 & 0.14596 \\ \mathrm{H} & 0.81183 & 1.76536 & 0.85844 \\ \mathrm{H} & -1.95473 & 1.60021 & 0.30393 \\ \mathrm{H} & -1.07762 & -0.86785 & 1.15035 \\ \mathrm{H} & -0.63540 & -1.01623 & -0.53955 \\ \mathrm{O} & -2.66443 & -0.86806 & -0.08299 \\ \mathrm{H} & -2.91975 & -0.53547 & -0.94712 \\ \mathrm{O} & 3.21771 & -0.31561 & -0.13573 \\ \mathrm{H} & 0.64063 & 1.88451 & -0.97271\end{array}$

IM-6T

$\begin{array}{llll}\mathrm{C} & -0.42527 & 1.09282 & -0.44124 \\ \mathrm{C} & 0.95566 & 1.08339 & 0.23186 \\ \mathrm{C} & 1.65475 & -0.25536 & 0.20467 \\ \mathrm{C} & -1.47343 & 0.29496 & 0.26404 \\ \mathrm{H} & 0.88591 & 1.36524 & 1.28487 \\ \mathrm{H} & -0.31449 & 0.74624 & -1.47610 \\ \mathrm{H} & -2.42024 & 0.74437 & 0.52301 \\ \mathrm{O} & -1.57480 & -1.04237 & 0.06156 \\ \mathrm{H} & -0.71378 & -1.40114 & -0.19021 \\ \mathrm{O} & 1.25037 & -1.29183 & -0.18353 \\ \mathrm{H} & 1.64289 & 1.79274 & -0.23322 \\ \mathrm{H} & -0.75515 & 2.13132 & -0.48861\end{array}$


TS-11T

$\begin{array}{lrrr}\mathrm{C} & 0.72605 & 0.96845 & 0.50142 \\ \mathrm{C} & -0.44187 & 1.47471 & -0.29745 \\ \mathrm{C} & -1.94777 & -0.09189 & -0.31974 \\ \mathrm{C} & 1.58781 & -0.02438 & -0.19922 \\ \mathrm{H} & -0.31753 & 1.57161 & -1.36820 \\ \mathrm{H} & 0.36344 & 0.55783 & 1.45447 \\ \mathrm{H} & 2.63238 & 0.16314 & -0.39065 \\ \mathrm{O} & 1.29091 & -1.35060 & -0.16182 \\ \mathrm{H} & 0.38336 & -1.48078 & 0.13084 \\ \mathrm{O} & -1.64770 & -1.00034 & 0.30239 \\ \mathrm{H} & -1.09727 & 2.20274 & 0.16460 \\ \mathrm{H} & 1.34457 & 1.83170 & 0.77429\end{array}$

TS-12T

$\begin{array}{lrrr}\mathrm{C} & 0.35402 & 0.35174 & 0.61075 \\ \mathrm{C} & -0.82467 & 0.92628 & -0.00677 \\ \mathrm{C} & -1.98965 & 0.13242 & -0.24995 \\ \mathrm{C} & 1.65227 & 0.42286 & -0.12856 \\ \mathrm{H} & -0.83308 & 1.96465 & -0.31506 \\ \mathrm{H} & 0.25673 & -0.48831 & 1.28413 \\ \mathrm{H} & 1.44182 & 1.04928 & 0.96222 \\ \mathrm{H} & 1.74127 & 1.14507 & -0.93040 \\ \mathrm{O} & 2.45414 & -0.67469 & -0.24101 \\ \mathrm{H} & 1.94416 & -1.46347 & -0.02341 \\ \mathrm{O} & -2.06034 & -1.05796 & 0.03627 \\ \mathrm{H} & -2.85313 & 0.65416 & -0.69235\end{array}$

$\mathrm{CO}+\mathrm{CH}_{2} \mathrm{CH}_{2} \mathrm{CHOH}$ (MP2, M06-2x DNC)

$\begin{array}{lrrr}\mathrm{C} & 1.30012 & 0.60879 & 0.63128 \\ \mathrm{C} & 1.07411 & 1.47257 & -0.56964 \\ \mathrm{C} & -2.65220 & 0.13378 & 0.70770 \\ \mathrm{C} & 1.51368 & -0.83277 & 0.28026 \\ \mathrm{H} & 1.72328 & 1.36858 & -1.43169 \\ \mathrm{H} & 0.45089 & 0.71987 & 1.32412 \\ \mathrm{H} & 1.96594 & -1.50782 & 0.99674 \\ \mathrm{O} & 0.55265 & -1.46149 & -0.46658 \\ \mathrm{H} & 0.15074 & -0.79677 & -1.03751 \\ \mathrm{O} & -2.34283 & 0.04066 & -0.38539 \\ \mathrm{H} & 0.42813 & 2.33967 & -0.51783 \\ \mathrm{H} & 2.18818 & 0.94880 & 1.18427\end{array}$

IM-7T

$\begin{array}{lrrr}\mathrm{C} & 0.55712 & 1.09120 & 0.42144 \\ \mathrm{C} & -0.78821 & 1.09588 & -0.22176 \\ \mathrm{C} & -1.63941 & -0.04688 & -0.19339 \\ \mathrm{C} & 1.56806 & 0.20052 & -0.25398 \\ \mathrm{H} & -1.14301 & 1.98031 & -0.73401 \\ \mathrm{H} & 0.42853 & 0.75159 & 1.46137 \\ \mathrm{H} & 2.59423 & 0.53234 & -0.31917 \\ \mathrm{O} & 1.43880 & -1.13881 & -0.14729 \\ \mathrm{H} & 0.52040 & -1.35709 & 0.09032 \\ \mathrm{O} & -1.30110 & -1.12567 & 0.29405 \\ \mathrm{H} & -2.64002 & 0.06043 & -0.63441 \\ \mathrm{H} & 0.95296 & 2.10387 & 0.44787\end{array}$


TS-13T (MP2, M06-2x DNC)

$\begin{array}{lrrr}\mathrm{C} & 0.54019 & 1.22430 & -0.14087 \\ \mathrm{C} & -0.92412 & 1.07440 & -0.08722 \\ \mathrm{C} & -1.61548 & -0.19977 & 0.06094 \\ \mathrm{C} & 1.48849 & 0.19612 & 0.36223 \\ \mathrm{H} & -1.51710 & 1.90572 & -0.45933 \\ \mathrm{H} & -0.30346 & 1.53641 & 0.87073 \\ \mathrm{H} & 2.44668 & 0.53837 & 0.73394 \\ \mathrm{O} & 1.54332 & -1.00513 & -0.25472 \\ \mathrm{H} & 0.60740 & -1.30058 & -0.31883 \\ \mathrm{O} & -1.08809 & -1.30318 & 0.06713 \\ \mathrm{H} & -2.71373 & -0.10364 & 0.14605 \\ \mathrm{H} & 0.90392 & 2.11994 & -0.64228\end{array}$

TS-14T

$\begin{array}{lrrr}\mathrm{C} & -0.75389 & 1.00332 & 0.42660 \\ \mathrm{C} & 0.28341 & 1.52091 & -0.27320 \\ \mathrm{C} & 1.89373 & -0.02132 & -0.08428 \\ \mathrm{C} & -1.69673 & 0.04295 & -0.16362 \\ \mathrm{H} & 0.92937 & 2.27316 & 0.16433 \\ \mathrm{H} & -2.76135 & 0.23089 & -0.15591 \\ \mathrm{O} & -1.40681 & -1.28816 & -0.08292 \\ \mathrm{H} & -0.45079 & -1.40182 & 0.00681 \\ \mathrm{O} & 1.59802 & -1.13953 & 0.12536 \\ \mathrm{H} & 2.91501 & 0.39996 & 0.07174 \\ \mathrm{H} & -0.82369 & 1.21742 & 1.49292 \\ \mathrm{H} & 0.30261 & 1.42682 & -1.35232\end{array}$

IM-8T

$\begin{array}{lrrr}\mathrm{C} & -0.41477 & 1.08426 & 0.41268 \\ \mathrm{C} & 0.96724 & 1.07933 & -0.14370 \\ \mathrm{C} & 1.62110 & -0.28512 & -0.14180 \\ \mathrm{C} & -1.50774 & 0.41478 & -0.28742 \\ \mathrm{H} & 1.63945 & 1.75980 & 0.38666 \\ \mathrm{H} & -2.39955 & 0.95445 & -0.57647 \\ \mathrm{O} & -1.75353 & -0.90114 & -0.03548 \\ \mathrm{H} & -0.91567 & -1.31177 & 0.22727 \\ \mathrm{O} & 1.07591 & -1.30767 & 0.17949 \\ \mathrm{H} & 2.67442 & -0.30379 & -0.47426 \\ \mathrm{H} & -0.54383 & 1.41302 & 1.44059 \\ \mathrm{H} & 0.97116 & 1.39929 & -1.19433\end{array}$

$\mathrm{HCO}+\mathrm{CH}_{2} \mathrm{CHCHOH}$

$\begin{array}{llll}\text { C } & -1.91527 & 0.64711 & 0.00024\end{array}$

$\begin{array}{llll}\text { C } & -0.87828 & 1.54967 & -0.00021\end{array}$

$\begin{array}{llll}\text { C } & 2.95848 & 0.45927 & -0.00036\end{array}$

$\begin{array}{llll}\text { C } & -1.80158 & -0.73037 & 0.00014\end{array}$

$\begin{array}{llll}\mathrm{H} & -1.07077 & 2.61080 & -0.00007\end{array}$

$\mathrm{H} \quad \begin{array}{llll}-2.66451 & -1.37844 & 0.00044\end{array}$

$\begin{array}{llll}\mathrm{O} & -0.64643 & -1.41508 & -0.00036\end{array}$

$\mathrm{H} \quad 0.11358 \quad-0.82014 \quad-0.00043$

$\begin{array}{llll}\text { O } & 2.16496 & -0.40135 & 0.00036\end{array}$

$\begin{array}{llll}\mathrm{H} & 4.06402 & 0.29586 & 0.00121\end{array}$

$\begin{array}{llll}\mathrm{H} & -2.93067 & 1.02276 & 0.00073\end{array}$

$\begin{array}{llll}\mathrm{H} & 0.15990 & 1.24652 & -0.00078\end{array}$ 
TS-16T

IM-10T

$\begin{array}{lrrrrrrr}\mathrm{C} & -0.27637 & 1.11431 & 0.35497 & \mathrm{C} & -0.33156 & 1.21778 & 0.34012 \\ \mathrm{C} & 1.14174 & 0.98449 & -0.12529 & \mathrm{C} & 1.09492 & 0.95280 & -0.15229 \\ \mathrm{C} & 1.59494 & -0.45837 & -0.10561 & \mathrm{C} & 1.51500 & -0.48516 & -0.10024 \\ \mathrm{C} & -1.32763 & 0.42794 & -0.34298 & \mathrm{C} & -1.36970 & 0.45613 & -0.40318 \\ \mathrm{H} & 1.84648 & 1.57825 & 0.46174 & \mathrm{H} & 1.83390 & 1.53145 & 0.41178 \\ \mathrm{H} & -1.32901 & 1.72094 & -0.22959 & \mathrm{O} & -1.91379 & -0.69763 & -0.02644 \\ \mathrm{O} & -2.05619 & -0.65895 & -0.04633 & \mathrm{H} & -1.20810 & -1.36416 & 0.03777 \\ \mathrm{H} & -1.41623 & -1.34251 & 0.21792 & \mathrm{O} & 0.79910 & -1.40827 & 0.19391 \\ \mathrm{O} & 0.88674 & -1.38968 & 0.17120 & \mathrm{H} & 2.57154 & -0.67908 & -0.36027 \\ \mathrm{H} & 2.65172 & -0.63213 & -0.38150 & \mathrm{H} & -0.41051 & 0.95226 & 1.39979 \\ \mathrm{H} & -0.44869 & 1.33003 & 1.40685 & \mathrm{H} & 1.20595 & 1.26931 & -1.19508 \\ \mathrm{H} & 1.25534 & 1.32430 & -1.16087 & \mathrm{H} & -0.52726 & 2.28817 & 0.25976\end{array}$

TS-17T

$\begin{array}{llll}\mathrm{C} & 0.40134 & 1.40906 & 0.56199 \\ \mathrm{C} & 1.37559 & 0.65168 & -0.25622 \\ \mathrm{C} & 1.19043 & -0.83903 & -0.26110 \\ \mathrm{C} & -1.51467 & 0.56518 & -0.72195 \\ \mathrm{H} & 2.41511 & 0.81455 & 0.07421 \\ \mathrm{O} & -2.04847 & -0.24465 & 0.09924 \\ \mathrm{H} & -1.44588 & -1.00296 & 0.29839 \\ \mathrm{O} & 0.39003 & -1.45297 & 0.40151 \\ \mathrm{H} & 1.89000 & -1.38924 & -0.91456 \\ \mathrm{H} & 0.04362 & 0.98866 & 1.48957 \\ \mathrm{H} & 1.35759 & 0.97829 & -1.29996 \\ \mathrm{H} & 0.29093 & 2.47034 & 0.41007\end{array}$

$\mathrm{HOC}+\mathrm{CH}_{2} \mathrm{CH}_{2} \mathrm{CHO}(\mathrm{B} 3 \mathrm{LYP}, \mathrm{M} 06-2 \mathrm{x}$ DNC)

$\begin{array}{lrrr}\mathrm{C} & 1.48909 & 1.46703 & 0.15130 \\ \mathrm{C} & 2.17522 & 0.18675 & -0.13464 \\ \mathrm{C} & 1.31454 & -1.05039 & -0.07329 \\ \mathrm{C} & -2.75720 & 1.17312 & -0.14261 \\ \mathrm{H} & 3.01307 & -0.00697 & 0.56178 \\ \mathrm{O} & -2.82354 & -0.43858 & -0.01011 \\ \mathrm{H} & -1.87225 & -0.64229 & 0.05276 \\ \mathrm{O} & 0.13732 & -1.07217 & 0.18540 \\ \mathrm{H} & 1.85487 & -1.99983 & -0.26295 \\ \mathrm{H} & 0.60992 & 1.48945 & 0.77620 \\ \mathrm{H} & 2.66255 & 0.18799 & -1.11877 \\ \mathrm{H} & 1.89166 & 2.39859 & -0.21599\end{array}$


TS-15T

IM-9T

$\begin{array}{lrrrrrrr}\mathrm{C} & -0.42791 & 1.12118 & -0.38344 & \mathrm{C} & -0.50027 & 1.03344 & -0.42858 \\ \mathrm{C} & 0.95995 & 1.10354 & 0.11766 & \mathrm{C} & 0.77752 & 1.09703 & 0.31207 \\ \mathrm{C} & 1.76579 & -0.22200 & 0.08657 & \mathrm{C} & 1.86211 & 0.00726 & 0.04951 \\ \mathrm{C} & -1.48248 & 0.32710 & 0.29980 & \mathrm{C} & -1.64750 & 0.21728 & 0.03804 \\ \mathrm{H} & 1.01315 & 1.33828 & 1.19135 & \mathrm{H} & 0.62444 & 0.99756 & 1.39711 \\ \mathrm{H} & -0.61857 & 1.41360 & -1.40768 & \mathrm{H} & -0.56909 & 1.50015 & -1.40085 \\ \mathrm{H} & -2.49995 & 0.71256 & 0.15724 & \mathrm{H} & -2.55760 & 0.36164 & -0.55482 \\ \mathrm{H} & -1.33270 & 0.29738 & 1.39386 & \mathrm{H} & -1.93812 & 0.49048 & 1.07734 \\ \mathrm{O} & -1.48011 & -1.00411 & -0.12061 & \mathrm{O} & -1.38380 & -1.13532 & 0.16427 \\ \mathrm{H} & 0.08415 & -1.22075 & -0.21064 & \mathrm{O} & 1.36294 & -1.15307 & -0.20492 \\ \mathrm{O} & 1.08846 & -1.29076 & -0.06365 & \mathrm{H} & 1.27914 & 2.04997 & 0.15560 \\ \mathrm{H} & 1.59503 & 1.83889 & -0.37357 & \mathrm{H} & 0.37697 & -1.22268 & -0.17536\end{array}$

TS-18T

$\begin{array}{lrllllll}\mathrm{C} & -0.74693 & 1.00816 & -0.43973 & \mathrm{C} & -1.44086 & 0.25228 & -0.40006 \\ \mathrm{C} & 0.30717 & 1.46560 & 0.29479 & \mathrm{C} & -1.36891 & 1.49662 & 0.04226 \\ \mathrm{C} & 2.00631 & 0.11605 & 0.05846 & \mathrm{C} & 1.91526 & 0.80639 & 0.64049 \\ \mathrm{C} & -1.62623 & -0.07316 & 0.07193 & \mathrm{C} & -1.00955 & -0.93782 & 0.40665 \\ \mathrm{H} & 0.33613 & 1.28743 & 1.36582 & \mathrm{H} & -0.97214 & 1.72134 & 1.02519 \\ \mathrm{H} & -0.87751 & 1.31423 & -1.46900 & \mathrm{H} & -1.82367 & 0.03908 & -1.39213 \\ \mathrm{H} & -2.59224 & -0.16343 & -0.43791 & \mathrm{H} & -1.82210 & -1.65180 & 0.58589 \\ \mathrm{H} & -1.89175 & 0.08778 & 1.13926 & \mathrm{H} & -0.65414 & -0.60544 & 1.40042 \\ \mathrm{O} & -1.04398 & -1.33763 & 0.12591 & \mathrm{O} & 0.08587 & -1.59940 & -0.10629 \\ \mathrm{O} & 1.54023 & -1.01290 & -0.16991 & \mathrm{O} & 2.04389 & 0.21765 & -0.46707 \\ \mathrm{H} & 0.89468 & 2.30636 & -0.04354 & \mathrm{H} & -1.69032 & 2.33133 & -0.56561 \\ \mathrm{H} & 0.51875 & -1.12813 & -0.11538 & \mathrm{H} & 1.34870 & -0.48531 & -0.60281\end{array}$




\section{M06-2x/cc-pVTZ Cartesian Coordinates for the Subsequent HCO/HOC Pathways of O $\left({ }^{3} \mathrm{P}\right)$ + 3-Butyn-1-ol}

\begin{tabular}{|c|c|c|c|c|c|c|c|}
\hline \multicolumn{4}{|c|}{$\mathrm{CH}_{2} \mathrm{CCH}_{2} \mathrm{OH}$} & \multicolumn{4}{|c|}{ TS-1D } \\
\hline $\mathrm{C}$ & -0.72566 & -0.59238 & 0.05232 & $\mathrm{C}$ & -0.69533 & -0.60211 & -0.06706 \\
\hline $\mathrm{C}$ & -1.65532 & 0.31943 & -0.02420 & $\mathrm{C}$ & -1.63571 & 0.36001 & -0.01350 \\
\hline $\mathrm{C}$ & 0.74930 & -0.63717 & -0.01248 & $\mathrm{C}$ & 0.69995 & -0.55885 & -0.08713 \\
\hline $\mathrm{H}$ & -2.70684 & 0.08164 & 0.08242 & $\mathrm{H}$ & -2.58501 & 0.21732 & 0.48202 \\
\hline $\mathrm{H}$ & -1.39995 & 1.36022 & -0.21881 & $\mathrm{H}$ & -1.51710 & 1.27298 & -0.59394 \\
\hline $\mathrm{H}$ & 1.06301 & -1.15702 & -0.91811 & $\mathrm{H}$ & 1.28526 & -1.39642 & -0.44471 \\
\hline $\mathrm{H}$ & 1.13900 & -1.20597 & 0.83805 & $\mathrm{H}$ & 0.07720 & -1.12934 & 0.91155 \\
\hline $\mathrm{O}$ & 1.32729 & 0.65621 & -0.07488 & $\mathrm{O}$ & 1.45767 & 0.56752 & 0.04988 \\
\hline $\mathrm{H}$ & 1.07659 & 1.13219 & 0.72165 & $\mathrm{H}$ & 0.86484 & 1.30094 & 0.25215 \\
\hline
\end{tabular}

IM-1D

$\begin{array}{lrrr}\mathrm{C} & -0.68283 & 0.61043 & -0.00004 \\ \mathrm{C} & -1.50649 & -0.48977 & 0.00003 \\ \mathrm{C} & 0.69860 & 0.59319 & 0.00004 \\ \mathrm{H} & -2.57891 & -0.37868 & -0.00011 \\ \mathrm{H} & -1.13353 & -1.50607 & 0.00032 \\ \mathrm{H} & 1.29466 & 1.49207 & 0.00013 \\ \mathrm{O} & 1.45161 & -0.52336 & 0.00002 \\ \mathrm{H} & 0.88123 & -1.29876 & -0.00051 \\ \mathrm{H} & -1.13199 & 1.59518 & -0.00016\end{array}$

TS-2D

$\begin{array}{llll}\text { C } & 0.61760 & 0.62605 & 0.05520\end{array}$

C $\quad 1.45563 \quad-0.53755 \quad-0.00096$

$\begin{array}{llll}\text { C } & -0.81598 & 0.68445 & 0.05820\end{array}$

$\mathrm{H} \quad 2.52340 \quad-0.45102 \quad 0.12070$

$\begin{array}{llll}\mathrm{H} & 1.07712 & -1.50494 & -0.29903\end{array}$

$\begin{array}{llll}\mathrm{H} & -0.01065 & 1.01779 & -0.99410\end{array}$

$\begin{array}{llll}0 & -1.42445 & -0.50348 & 0.00313\end{array}$

$\mathrm{H} \quad-0.83073 \quad-1.24736 \quad 0.20030$

$\begin{array}{llll}\mathrm{H} & 1.09301 & 1.57568 & 0.27240\end{array}$ 
IM-2D

$\begin{array}{lrrr}\mathrm{C} & -0.61840 & 0.71216 & 0.00018 \\ \mathrm{C} & -1.39377 & -0.55312 & 0.00010 \\ \mathrm{C} & 0.91531 & 0.66652 & 0.00015 \\ \mathrm{H} & -1.71423 & -1.01563 & -0.92173 \\ \mathrm{H} & -1.70733 & -1.01987 & 0.92219 \\ \mathrm{H} & -0.85388 & 1.33557 & 0.86829 \\ \mathrm{O} & 1.37879 & -0.54432 & -0.00036 \\ \mathrm{H} & 0.68055 & -1.23473 & -0.00086 \\ \mathrm{H} & -0.85418 & 1.33591 & -0.86754\end{array}$

$\mathrm{HOC}+\mathrm{C}_{2} \mathrm{H}_{4}$

$\begin{array}{llll}\text { C } & -1.30421 & 0.74140 & -0.00001\end{array}$

$\begin{array}{llll}\text { C } & -1.61833 & -0.54945 & 0.00001\end{array}$

$\begin{array}{llll}\text { C } & 1.81980 & 0.63455 & -0.00001\end{array}$

$\begin{array}{llll}\mathrm{H} & -1.77504 & -1.09354 & -0.92315\end{array}$

$\begin{array}{llll}\mathrm{H} & -1.77503 & -1.09352 & 0.92318\end{array}$

$\mathrm{H} \quad \begin{array}{lll}-1.16541 & 1.29402 & 0.91983\end{array}$

$\begin{array}{llll}\text { O } & 1.49858 & -0.58105 & 0.00000\end{array}$

$\begin{array}{llll}\mathrm{H} & 0.50867 & -0.71148 & 0.00001\end{array}$

$\begin{array}{llll}\mathrm{H} & -1.16542 & 1.29400 & -0.91986\end{array}$
TS-3D

$\begin{array}{lrrr}\mathrm{C} & -0.89853 & 0.70976 & -0.00001 \\ \mathrm{C} & -1.36140 & -0.58818 & 0.00001 \\ \mathrm{C} & 1.10414 & 0.68774 & 0.00001 \\ \mathrm{H} & -1.58415 & -1.10729 & -0.92249 \\ \mathrm{H} & -1.58414 & -1.10727 & 0.92253 \\ \mathrm{H} & -0.95412 & 1.29963 & 0.90773 \\ \mathrm{O} & 1.42641 & -0.51700 & -0.00001 \\ \mathrm{H} & 0.60002 & -1.10460 & -0.00002 \\ \mathrm{H} & -0.95412 & 1.29960 & -0.90777\end{array}$

\section{$\mathrm{CHCHCH}_{2} \mathrm{OH}$}

$\begin{array}{llll}\text { C } & -0.73793 & -0.09856 & 0.38745\end{array}$

$\begin{array}{llll}\text { C } & -1.82902 & -0.04261 & -0.32460\end{array}$

$\begin{array}{llll}\text { C } & 0.55552 & 0.57139 & 0.00535\end{array}$

$\mathrm{H} \quad \begin{array}{llll}-2.83473 & -0.42488 & -0.26417\end{array}$

$\begin{array}{llll}\mathrm{H} & -0.71125 & -0.67745 & 1.31234\end{array}$

$\begin{array}{llll}\mathrm{H} & 0.84315 & 1.28669 & 0.77638\end{array}$

$\mathrm{H} \quad 0.42403 \quad 1.11666 \quad-0.93300$

$\begin{array}{llll}\text { O } & 1.61981 & -0.35776 & -0.07163\end{array}$

$\begin{array}{llll}\mathrm{H} & 1.38895 & -1.02025 & -0.72775\end{array}$ 
TS-4D

$\begin{array}{llll}\mathrm{C} & -0.67643 & -0.29889 & 0.15927 \\ \mathrm{C} & -1.96523 & 0.06506 & -0.32299 \\ \mathrm{C} & 0.54889 & 0.46369 & 0.03584 \\ \mathrm{H} & -2.86291 & 0.10923 & 0.28026 \\ \mathrm{H} & -0.48067 & -1.37272 & 0.28124 \\ \mathrm{H} & -0.43338 & 0.15005 & 1.22856 \\ \mathrm{H} & 0.54646 & 1.54232 & 0.03133 \\ \mathrm{O} & 1.76414 & -0.09876 & -0.08680 \\ \mathrm{H} & 1.67403 & -1.01795 & -0.35971\end{array}$

TS-5D

$\begin{array}{lrrr}\mathrm{C} & 0.51303 & 0.34278 & 0.06902 \\ \mathrm{C} & 1.87079 & -0.07211 & -0.09219 \\ \mathrm{C} & -0.58040 & -0.56803 & -0.02369 \\ \mathrm{H} & 2.67170 & 0.64814 & -0.08240 \\ \mathrm{H} & 0.31746 & 1.42361 & 0.10399 \\ \mathrm{H} & 2.09703 & -1.12381 & -0.17534 \\ \mathrm{H} & 0.09929 & -0.32421 & 1.10467 \\ \mathrm{O} & -1.78430 & 0.02189 & -0.06017 \\ \mathrm{H} & -1.73157 & 0.98534 & -0.18834\end{array}$

IM-3D

$\begin{array}{llll}\mathrm{C} & -0.58334 & -0.38293 & 0.00003 \\ \mathrm{C} & -1.87904 & 0.08229 & 0.00001 \\ \mathrm{C} & 0.53677 & 0.41764 & 0.00003 \\ \mathrm{H} & -2.71600 & -0.59698 & -0.00020 \\ \mathrm{H} & -0.42043 & -1.45834 & 0.00007 \\ \mathrm{H} & -2.08990 & 1.14245 & -0.00004 \\ \mathrm{H} & 0.48400 & 1.49674 & 0.00007 \\ \mathrm{O} & 1.81058 & -0.03596 & -0.00005 \\ \mathrm{H} & 1.81125 & -0.99825 & 0.00009\end{array}$

IM-4D

$\begin{array}{lrrr}\mathrm{C} & 0.50727 & 0.42108 & 0.21583 \\ \mathrm{C} & 1.80307 & -0.17407 & -0.19402 \\ \mathrm{C} & -0.62025 & -0.57180 & 0.21439 \\ \mathrm{H} & 2.63033 & 0.45506 & -0.47987 \\ \mathrm{H} & 0.27809 & 1.34064 & -0.35219 \\ \mathrm{H} & 1.95584 & -1.23531 & -0.08030 \\ \mathrm{H} & 0.54256 & 0.72742 & 1.27915 \\ \mathrm{O} & -1.73639 & -0.03120 & -0.16895 \\ \mathrm{H} & -1.65634 & 0.91054 & -0.43241\end{array}$


TS-6D

$\begin{array}{llll}\mathrm{C} & 0.94457 & 0.63805 & -0.27519 \\ \mathrm{C} & 1.41216 & -0.59282 & 0.06814 \\ \mathrm{C} & -0.82757 & 0.35960 & 0.66787 \\ \mathrm{H} & 1.19666 & -1.46666 & -0.53077 \\ \mathrm{H} & 0.55649 & 0.82027 & -1.27347 \\ \mathrm{H} & 1.94068 & -0.74993 & 0.99681 \\ \mathrm{H} & 1.26388 & 1.51797 & 0.26348 \\ \mathrm{O} & -1.58516 & -0.30460 & -0.14143 \\ \mathrm{H} & -1.45143 & -0.11380 & -1.08951\end{array}$

$\mathrm{HOC}+\mathrm{C}_{2} \mathrm{H}_{4}$

$\begin{array}{llll}\text { C } & 1.30400 & 0.74143 & 0.00010\end{array}$

$\begin{array}{llll}\text { C } & 1.61790 & -0.54946 & 0.00009\end{array}$

$\begin{array}{llll}\text { C } & -1.81961 & 0.63449 & -0.00001\end{array}$

$\mathrm{H} \quad 1.77557 \quad-1.09331 \quad-0.92304$

$\begin{array}{llll}\mathrm{H} & 1.16637 & 1.29432 & -0.91976\end{array}$

$\begin{array}{llll}\mathrm{H} & 1.77342 & -1.09382 & 0.92329\end{array}$

$\begin{array}{llll}\mathrm{H} & 1.16413 & 1.29378 & 0.91993\end{array}$

$\begin{array}{llll}\text { O } & -1.49814 & -0.58104 & -0.00012\end{array}$

$\begin{array}{llll}\mathrm{H} & -0.50812 & -0.71135 & -0.00049\end{array}$

\section{$\mathrm{CH}_{2} \mathrm{CH}_{2} \mathrm{CHO}$}

$\begin{array}{llll}\mathrm{C} & -1.44196 & -0.56656 & 0.00008 \\ \mathrm{C} & -0.63131 & 0.67044 & -0.00008 \\ \mathrm{C} & 0.85685 & 0.43461 & 0.00006 \\ \mathrm{H} & -0.84957 & 1.31412 & 0.86427 \\ \mathrm{O} & 1.37422 & -0.64638 & 0.00001 \\ \mathrm{H} & 1.47431 & 1.35265 & 0.00019 \\ \mathrm{H} & -0.95233 & -1.52668 & -0.00053 \\ \mathrm{H} & -0.84934 & 1.31375 & -0.86480 \\ \mathrm{H} & -2.51830 & -0.51371 & 0.00042\end{array}$

TS-7D

$\begin{array}{lrrr}\mathrm{C} & -1.64189 & -0.56047 & 0.00591 \\ \mathrm{C} & -1.07034 & 0.66273 & -0.00716 \\ \mathrm{C} & 1.15107 & 0.43312 & -0.00001 \\ \mathrm{H} & -0.98619 & 1.24149 & 0.90486 \\ \mathrm{O} & 1.66901 & -0.61534 & -0.00373 \\ \mathrm{H} & 1.68887 & 1.41417 & 0.03935 \\ \mathrm{H} & -1.84356 & -1.07728 & 0.93341 \\ \mathrm{H} & -0.99199 & 1.22512 & -0.92959 \\ \mathrm{H} & -1.85222 & -1.09308 & -0.91063\end{array}$




\begin{tabular}{lrrr}
\multicolumn{4}{c}{$\mathrm{HCO}+\mathrm{C}_{2} \mathrm{H}_{4}$} \\
$\mathrm{C}$ & 1.52671 & -0.67841 & -0.02275 \\
$\mathrm{C}$ & 1.59895 & 0.62931 & 0.16563 \\
$\mathrm{C}$ & -1.49401 & 0.33878 & -0.53962 \\
$\mathrm{H}$ & 2.32959 & 1.23774 & -0.35136 \\
$\mathrm{O}$ & -1.87329 & -0.18003 & 0.43757 \\
$\mathrm{H}$ & -1.06000 & -0.19976 & -1.41899 \\
$\mathrm{H}$ & 2.19816 & -1.19226 & -0.69861 \\
$\mathrm{H}$ & 0.93267 & 1.14006 & 0.84953 \\
$\mathrm{H}$ & 0.79599 & -1.28363 & 0.49931
\end{tabular}

